UC-NRLF

\|\|\|\|\|\|$|\|\mid\| \|$

$\begin{array}{lll}5 & 35480\end{array}$ 
LIBRARY

OF THE

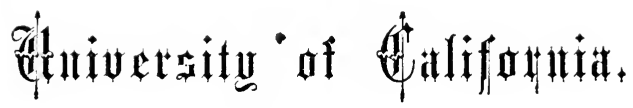

No. 6664

Division

Range

Shelf

Received July

1870 


$*$ 


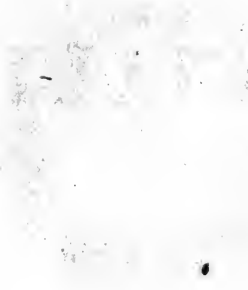

ำ - 


\section{UNITY OF PURPOSE,}

OR

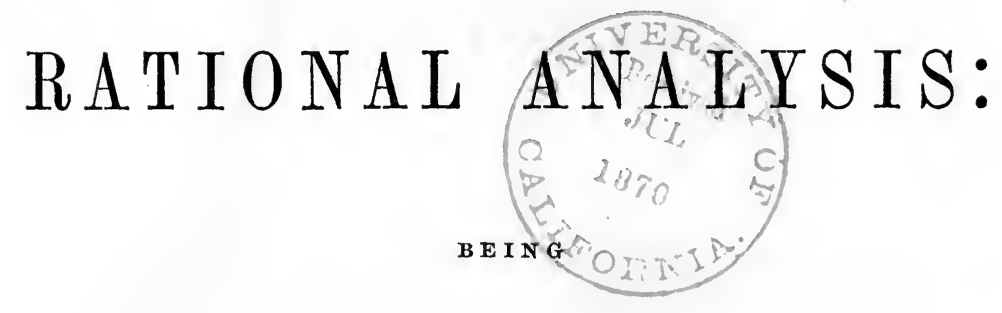

A T R E A T I E

DESIGNED

TO DISCLOSE PHYSICAL TRUTHS, AND TO DETECT AND EXPOSE POPULAR ERRORS.

\section{BY AUGUSTUS YOUNG.}

"These are not, perhaps, very attractive speculations; they disturb old and favorite associations; they serve to reduce many cherished traditions, much painfully acquired knowledge, to obsolete lore; but these things are so, and we must accustom ourselves to regard them and their consequences without shrinking."

B O S T O N :

PRINTED BY S. N. DICKINSON \& CO. 1846 . 
Entered according to Act of Congress, in the year 1846, by A UGUSTUS YOUNG,

In the Clerk's Office of the District Court of the District of Vermont. 


\section{0 N T E N T S.}

[Although in the work, some of the respective Chapters are divided into, or are composed of several Sections, such division will be disregarded in the Table of Contents, - the subject matters of the Chapters only being given.]

Page.

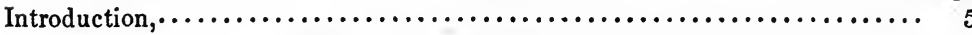

CHA P T E R I.

On the Quadrature of the Circle, $\ldots \ldots \ldots \ldots \ldots \ldots \ldots \ldots \ldots \ldots \ldots \ldots \ldots \ldots$

CHAPTER II.

On the Law of Gravity, and the Popular Deduction and Promulgation of the

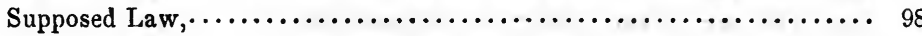

CH A P TE R III.

Of the Proper Elements from which to determine the Laws of Force and

Motion, incident to the Heavenly Bodies, in their Eternal Rounds,.... 198

CH A P T E R IV.

On the Hypothesis suggested by Kepler in respect to Elliptical Orbits, .... 217

C H A P T E R V.

Outline of a Theory of the Solar System, $\ldots \ldots \ldots \ldots \ldots \ldots \ldots \ldots \ldots \ldots \ldots \ldots \ldots \ldots \ldots \ldots$

CHAPTER VI.

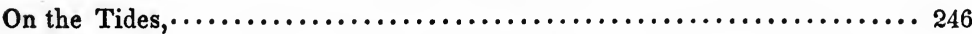

CHAPTER VII.

An Investigation of the Theory of the Progressive Motion of Light, $\ldots \ldots \ldots 261$ 
Page 37, line 33, for may read might.

Page 48, line 35, before the word propose, read do not.

Page 48, line 42, for divisions, read divisors.

Page 58, line 43, before the word will, read when equated.

Page 60, line 19, before the word constantly, read when equated, will.

Page 97, line 11, for space, read spaces.

Page 100, line 37, erase the word same commencing the line.

Page 107, line 14, for form, read force.

Page 116, line 33, erase the words the square root of.

Page 117, line 3, for minutes, read seconds,

Page 131, line 25, read the word we after the word $i t$, at the end of the line.

Page 131, line 27, erase the word we commencing the line.

Page 210, line 6, for or, read is.

Page 216, line 10, for time, read line.

Page 224, line 30, for distance, read diameter.

Page 264, line 16, for sutor, read sector.

Page 286, line 17, for doctrines, read dictums. 


\section{N T R O D U C T I $0 \mathrm{~N}$.}

ThE object of this work as declared in the title page, is to elicit inquiry into, or reëxamination of certain matters which the world at large now receive implicitly, at the hands of others, as scientific truths; but which, perhaps, upon a more rigid and scrupulous examination, divested of a too easy faith in matters purely scientific, may be found to be but popular errors, which should be eradicated for the benefit of physical science; and should the fact be disclosed that many matters which are now almost universally esteemed sublime scientific truths, are but dark and occult errors, the inquiry will naturally suggest itself, whether it may not have been found necessary to clothe them in a mathematical dress so wholly incomprehensible to the mass of mankind, as to make it a hopeless task from want of leisure and other facilities, to investigate the truth or falsehood promulgated by the learned through the medium of what is so triumphantly termed the higher branches of mathematics; and hence, whether mankind in general, have not been compelled to remain ignorant of those supposed physical truths, except by a confidential faith in those who profess to teach those things. And should my labors in anywise serve to induce the learned to stoop a little more to the necessities of the multitude, who lack leisure and opportunity to acquaint themselves with all the modern devices of mathematical science, my object will be fully accomplished.

I am aware that many will consider the subject matters of which I treat, (namely, the cold, material laws of nature,) as altogether grovelling in comparison with those flights of human imagination, which flood the world in such profusion, and which so liberally dispense to mankind the benefits of modern literature by means of novels and tales of fiction; and which are conceived by many to be paramount to every thing intellectual beside: but to fulfil a task so desirable to many, I confess I was never 
properly fitted and prepared; the preparation for it must, in general, come through the medium of a collegiate education and discipline. Besides, I have ever looked upon the universe as a reality, and not as a fiction; nor am I without hope, that eternal truths may yet become as pleasing to a great portion of mankind as is human error. Such is certainly the case to a very great extent in the moral world, and it is certainly too manifest to be overlooked or disregarded, that if a proper regard were had to that unity of purpose in which the Christian religion is founded, the world of mankind might be much more readily or easily squared and quadrated into what is befitting a moral world, than they could be equated to the same by means of Jewish rites and ceremonies, or by heathen philosophy. It would seem then to be but following out the principles of Christian philsosophy, to take example from that great dispensation of Divine truth in other departments of science, or in the natural world, by endeavoring to adopt that unity of purpose which alone seems calculated to lead to those truths which are to benefit mankind. If such, then, were our foundation, - whatever errors and mistakes might occur in our attempts to extend its benefits, - we should not be left without a land-mark, a witness, contained in such unity of purpose, whereby to correct those errors.

Shall we not, then, in our researches for truth, endeavor to follow out the true principles of Christian philosophy, with a view to some unity of purpose, - as being the most probable condition upon which we may hope for success?

In my pursuit after truth, I shall no more be satisfied with taking a middle flight, than Milton was in the sublimation of fiction; and as this must often bring me in contact with those who have attempted the most daring flights in the scientific world, (especially in the departments of mathematics and astronomy,) I shall speak of them and their doings, with a freedom and boldness which I suppose to be justly due to the subject, and to mankind; nor will $I$, in this respect, ask from the world other mercy than such as I show to others.

It may surprise the admiration which many have been taught to entertain of Sir Isaac Newton, - who by his flattering biog. raphers, has been styled the creator of natural philosophy,that I should place Kepler on far higher ground as a philosopher, so far, at least, as science has been benefited by their researches or hypotheses. It may surprise many that I should esteem Kepler as the father of physical astronomy; and assert that he has done more in laying the foundation of that sublime science in those eternal truths upon which its superstructure 
should be erected, than all others; and that, had a like sagacity succeeded him in those who attempted its superstructure, its strength and beauty would have been grand indeed in comparison with its present deformity. And although I shall treat of Kepler's elliptical hypothesis with something more than doubts, nevertheless, if it were an error in him, it could not have been the cause of errors committed by others in respect to the laws of force and motion, by which the phenomena of the heavenly bodies can only be explained.

Sir Isaac Newton lived in a day of problems, - in a time of great anxiety in respect to the science of astronomy, which had recently been ushered forth to the world, and somewhat advanced by the labors and researches of Copernicus, Tycho, Kepler and Galileo; and hence, the world then required some master spirit to collect the disjointed parts, and to raise the superstructure to their implicit admiration and content. Newton also lived in a country where science and the arts were better patronized by national pride than in any other country at the time; and he seems to have been fully able to avail himself of all advantages which came in his way; for, if we are to believe his admiring biographers, he was often enabled to keep his own discoveries a profound secret until they were disclosed to the world by others, although he never failed to assert his claim as the discoverer, and to have his claim allowed by his country; as in the case of Kepler, Liebnitz and Flamstead; although modern disclosures in respect to the treatment of Flamstead, have caused even high toned Englishmen to declare, that the name of Newton was no longer to be revered. And whether the doubt expressed in respect to Sir Richard Arkwright, may not with equal propriety be applied to Sir Isaac Newton, is for a candid world to determine, namely, "some esteem this remarkable man as a genius of the first order, gifted with an extraordinary power of invention; others, as an artful contriver, who understood how to appropriate to himself the discoveries of others."

But the biographer of Newton was certainly acquainted with what would induce implicit faith, and flatter a nation's vanity, when he saw fit to insert the pitiful story about the fall of the apple from the apple-tree, when Newton was sitting alone under the tree; and what Sir Isaac Newton asked himself on that occasion; and also what he said to his pet dog Diamond, on a certain occasion;- how the fall of the apple suggested to the mind of Sir Isaac, the hypothesis of the universal gravity of inert matter; - how Sir Isaac Newton devised a method for ascertaining whether gravity extended from the earth to the moon, and what would be the law of gravity in respect to distance;- 
and how his ecstatic agony, on supposing his conjecture about to be realized by proof, became so great as, to deprive him of the power to finish out the problem and final result, which was thenceforth to become the great physical law of the universe; and was therefore compelled to submit the finishing stroke to another person.

But such stuff cannot well be accounted for upon any other principle than that of a design to clothe Sir. Isaac Newton with something superhuman in the eyes of the world, and make him an object of adoration; and thereby to induce a belief, that such wonders could have been performed by no other. And such efforts have had their effect; Newton has been immortalized, and national pride has been gratified, however true science may have been retarded in consequence.

The world has generally supposed that Newton first disclosed the idea of attraction among the heavenly bodies; and that such attraction was the cause of the tides; notwithstanding, Kepler, some forty years previous to the advent of Newton's apple, had not only fully declared the principle to the world, but had done it in such a way and manner, as not to lead mankind into those atheistical and abhorrent notions, which are taught in the Newtonian philosophy in respect to the hypothesis. Kepler had declared that the tides were caused by the attraction of the moon; that the earth and moon mutually attract each other; and that, in consequence, they would come together, and that too, at a point as much nearer the earth, as the earth is greater than the moon, were they not prevented by the motion of the moon in its orbit. And a better development or declaration for the future progress of science, could not have been made. It was in nowise calculated to lead into error; it did not assume any particular hypothesis in respect to universal gravity, or attempt to declare its laws in respect to distance, but left them for future investigation, which long ere this, should have been both rational and conclusive.

It is said of Newton, that, after receiving the hint from the falling apple, setting out with the law of Kepler, namely, "that the squares of the times of revolution of the different planets, are proportioned to the cubes of their distances from the sun," he found by calculation, that the force of solar attraction or gravity, decreases proportionally to the square of the distance.

But I propose to show that if he set out with Kepler's said law, he very soon lost sight of it; as he did also of the whole spirit and meaning of Kepler's other law, which teaches that the motion of a planet in an eccentric orbit, varies as the distance of the planet from the sun varies; which last great law of Kepler, 
Newton must have wholly misunderstood or misapprehended; else the error aforesaid could not have been committed; for I shall endeavor to prove, that neither Sir Isaac, nor any other person, ever found the force of solar gravity to decrease proportionally to the square of the distance. For, if such be the case, Kepler's second law should be so far altered from the manner in which he declared it, as to assert that the motion of a system of planets must vary proportionally to each other, as their respective distances vary from the sun, thereby wholly overthrowing the great law of Kepler, with which Newton set out, as it would cause the squares of the periods of a system of planets to be as the fourth powers of their mean distances from the sun,-as may be readily ascertained by the most obtuse intellect. For a fact more easily ascertained scarcely exists, than that the mean motions of a system of planets are inversely as the square roots of their mean distances from the sun, which is the only possible condition in which the squares of the periods can be as the cubes of their mean distances from the sun.

Another fact as simple, is, that if the mean motions of a system of planets were inversely as their mean distances from the sun, and also, if the motion of each individual planet varies inversely as its distance varies during its period or revolution, then, indeed, the force of solar attraction would be inversely as the square of the distance; and the periods would be as the squares of their mean distances from the sun; and upon no other condition whatever could the force of solar attraction be inversely as the square of the distance.

. It is also said by Newton's biographer, that having discovered the law of gravity of the planets toward the sun, he endeavored to apply it to the moon, by comparing the deflection of the moon from a tangent to its orbit, with the law of falling bodies near the earth; and that, after many years of doubt, he at length found that the attractive power of the earth varied inversely as the square of the distance from its centre to the moon; and that after two years more, spent in penetrating the consequences of this discovery, his immortal Principia came forth, of which it is said, that not more than two or three of his contemporaries were capable of understanding it, and that more than fifty years elapsed before the great physical truth which it contained, (namely, his law of gravity,) was thoroughly understood by scientific men generally. Nor, indeed, was this accomplished, until a reconciliation to the law was brought about by Clairaut, by one of the strangest devices of which ingenuity, grown desperate, is capable, and which has produced a kind of Gordian knot in the 
science of astronomy, which we had much better cut than to pother much in attempting to untie it.

But perhaps a better commentary can scarcely be made upon our inclination to adore something which we know not, than that contained in a prospectus, issued in the city of New York,-by a respectable publisher, this year, (1846) for publishing in this country, for the first time, Newton's Principia, - in which the publisher remarks as follows:

"It is a singular fact that this remarkable work,- the Principia of Sir Isaac Newton, - the foundation, at once, of his worldwide fame, and the grandest monument of human intellect, should have remained comparatively unknown in this country to the present day. Singular, because the name of the immortal author is familiar as a household word; - himself honored, revered, looked up to as one of the demi-gods of by-gone times," \&c. " But the Principia has hitherto been inaceessible to popular use. A few copies in Latin, and occasionally one in English, were and are to be found in some of our large libraries, and in the possession of some loving disciple of the great master. A dead language, however, in the one case, and an enormous price in both, particularly in the ease of the English edition, have thus far opposed very sufficient obstacles to any general circulation of the work," \&c.

And such is the blind idolatry of a world, thus worshipping a fellow-mortal, which it may, at some future time, wish to make the scape-goat of its errors.

But of the law of gravity, I propose to treat in its proper place; remarking here, however, that, notwithstanding Sir Isaae Newton, Bernoulli, and others, have strangely endeavored to equate and adjust the respective attractive powers of the sun and moon, by calculating their respective influences in producing the tides,in which Newton estimates the influence of the moon to that of the sun as 3.5 to 1 ; Bernoulli as 2.5 to 1 , and M. Thouroud far less than either, - nevertheless, the earth revolves farther in its orbit in one day, about the sun, than the moon does about the earth, in an entire revolution; which circumstance ought to be accounted for in some rational and proper manner.

But Sir Isaac Newton having determined his law of gravity, physical consequents must necessarily follow or flow from it; as in the case of the motion of the moon's apogee, for instance; and although Sir Isaac, by his law of gravity, had furnished but just one fourth of the requisite quantity, or only sufficient to produce just one half of the actual or observed motion, yet Clairaut, at length, found out a way to make that answer.

So also in respect to the precession of the equinoxes, it is still 
insisted that Sir Isaac Newton assigned the true physical cause, namely: the attraction of the sun upon the surplus matter of the earth, over and above its greatest inscribed sphere; although Mr. Vince says, "it is acknowledged that Newton fell into an error in his investigations as to the amount of the effect." But this acknowledgment seems rather to have arisen from the necessity that the celebrated astronomer, Dr. Bradley, was under, of having a place to stand on, in respect to his discovery of the nutation of the poles of the earth. And in respect to the theory of the tides, I think Sir Isaac Newton has lost nothing from La Place's modification of the cause and effect, as he has left it still more absurd and inexplicable, if possible, than he found it.

As my principal purpose is to treat of, or inquire into the nature and effect of the more direct physical causes by which the worlds are more immediately governed and controlled in their eternal rounds, and as Sir Isaac Newton's theory of light does not so essentially interfere with or affect those laws, I shall omit to treat of that department in his philosophy, as that may be better borne with, however absurd it may be, than his theory and law of gravity, till a better philosophy shall correct the absurdities. But not so in respect to his theory of universal gxavity, (as by him taught to a too confiding world,) which, like some noxious plant or vine, has interwoven itself into the whole fabric of astronomy, disjoining its parts, hiding its beauties, and affecting the operations of all its physical laws.

This is the theory which, while it asserts that all gross or tangible matter in the universe, is inert, dead, and regardless, asserts also, that all matter is equally endued with an innate or inherent principle for the attraction of other matter; and hence, that inert matter is made to possess innate powers and innate terdencies. This is the theory which requires the heavenly bodies to be projected in the direction of a tangent to the orbit in which they are to revolve; - not that the planet is to pass in the direction in which it is projected; the projection being only one of the resulting forces, the other being inherent in inert matter, constantly operating, though at a vast distance, and that also through space, and which must forever after, take the whole charge of the body so projected, and safely guide it through all the disturbing forces, during its eternal rounds. This is the theory which has converted the whole universe into a system of disturbing forces, and the whole corps of astronomers into an exploring party, whose business it is to search out and regulate disturbances. And it would necessarily be so, if the theory were true; for the sun would then so disturb the original projectile force, that the planet could not pass - at all in the line of prujec- 
tion, and the planets, in turn, would disturb their neighbors, together with every stranger that came into their vicinity. Nevertheless, the disturbances are mutual, and not at all prevented by the inertia of matter.

But, to me, this seems quite too fortuitons, if not absolutely atheistical; and, therefore, I must leave it to others to suppose that a universe, governed and controlled by no other power than that arising from disturbing forces, would operate better than one governed and controlled by immutable laws of order and regularity.' Nevertheless, I do not accuse Sir Isaac Newton of entertaining atheistical views or notions, for he even wrote upon the prophecies of Daniel, and upon the Apocalypse, and were he now living, would probably be the best expounder extant, of their mysteries.

And finally, it is this theory, which, from the time of its promulgation, has served (with the world) "to make the worse appear the better reason, to perplex and dash maturest counsels," whereby astronomers have appeared to lose sight of first principles, and rather implicitly to follow the persuasive suggestions of a file leader, with a zeal that would have been far better. employed in the ways of truth, and in its development to the world.

And now, if it should be thought that I have been somewhat severe in my remarks, it ought also to be understood that $I$ have much idolatry to contend with, and even an apparent determination neither to behold nor acknowledge any error in anything Sir Isaac Newton may have presumed to teach, promulgate or find; and hence we so often find the naked allegation,- - even in the highest authorities, as if it were "confirmation strong as proofs of holy writ,"-that Sir Isaac Newton found, \&c.

But perhaps what I have said may only serve to show that my faith in the infallibility of Sir Isaac Newton, is not so great as that of some others; and if a further expression of such want of faith should be required, I will here declare, that I have yet to learn of a single beneficial discovery or principle, originally promulgated by Sir Isaac Newton, in anywise beneficial to physical astronomy.

But I will not attempt to justify raillery, or irreverence upon the ground that others before me have used such weapons. Nevertheless, Sir Richard Phillips, (who, perhaps, has made rather a feeble attack upon the stronghold, and who, perhaps, should have adduced more efficient evidence to justify his provocation,) in speaking of the Newtonian method of deducing the law of gravity from a consideration of the deflection of the moon from a tangent to its orbit, speaks in this wise:

"How gross the imposture, then, of founding a system on a 
fictitious fall of sixteen feet per minute!" \&c.--" How juggling, too, to compare a paper fall at the moon, with a real fall at the earth; and how delusive, even under that juggle, to take a minute when even the juggle itself would hold for no other time!" \&c. "But the wonder, after all, is far less, that a system-maker should be seduced to make the assertion, than that mathematicians, and men of moral worth should, from 1687 to 1831, have been mistaken enough to believe and teach such nonsense with solemn emphasis."

The fact is, that Sir Richard, and also thousands of others, perceived that there was discrepancy and error in the Newtonian promulgation of the law of gravity; and strange indeed have been the devices and attempts to correct or set aside the error; as also to reconcile the law with observed phenomena, in lieu of reinvestigating the Newtonian method, and thereby, at once, detecting the error which Sir Isaac committed.

But to me, Sir Isaac Newton's thoughts often appear to have been extremely crude and unanalyzed, and in them he often forgot his own philosophy, such as it was ; as, for instance, when he wished to be wiser than Galileo, in respect to the direction a body would take in its fall from a state of rest towards the earth, - he states that in some few hundred feet fall, it would advance some half inch eastward, in consequence of the diurnal motion of the earth; and some modern experimenters, (believing such to be the law,) have conceived that they have detected the half inch advance ; but a more whimsical error could not have been advanced, if we suppose the law of gravity to be an omnipresent law. But we are too ready to transgress against reason, if we suppose the law to be with us.

Unity of purpose in the Divine Architect of the universe, is the hypothesis in which I would involve all philosophy in respect to cause and effect; nor would I be too hasty in adopting conclusions which might savor of fortuity, instead of being the result of general and immutable laws. And whether some philosophers of high repute, since the days of Copernicus, Galileo, and Kepler, have sufficiently discarded fortuity or chance from being co-workers in the operations of nature, is a subject worthy of consideration; and if not, it would not be a wonder if, thereby, the universe had been thrown a little out of gear; and if it should, in consequence, require much equating to enable it to fulfil what would seem to be its proper operations or destination.

The Copernican system of astronomy was not only the result of great sagacity, at a time when much ignorance prevailed upon the science of astronomy; but, also, of a boldness bordering on temerity, in venturing to declare this theory to a world 
full of superstition and bigotry, and wedded, as the world always is, to those notions which have long been cherished by the multitude as matters of implicit faith.

Galileo did excellent service to the science of astronomy, not only in his discoveries by aid of the telescope, but in his other philosophical researches; and especially, in ascertaining and determining the law of falling bodies, near the earth.

But Kepler seems to have been the first who entertained rational notions of physical astronomy; at least, the first who obtained any knowledge of those immutable laws, based upon or founded in the immutability of numbers, by which the solar system is governed in its harmonious movements; which laws, so by him discovered, from their great utility to the science of astronomy, are, in great justice to their discoverer, called Kepler's laws. And happy would the circumstance have been for the science of astronomy, had those who succeeded those masterbuilders, and essayed to erect the superstructure, been careful to square their work by the foundation so skillfully and adrnirably laid.

Those master-builders did not assume to build upon hypothesis alone, but upon well-ascertained and demonstrated facts. Thus Kepler,- - in announeing to the world the law of gravity,did not attempt to announce a specific law, by which matter operates upon matter, by means of an equal, innate, or inherent gravity; nor did he aftempt to promulgate a law, by which matter operates upon matter, through distance or space; and hence his announcement was not calculated to mislead mankind in respect to a naked hypothesis, nor to cause them to err by an erroneous, pretended demonstration, either of which, to be sustained, would require the laws of nature to give way, or at least to subscribe liberally to their support, to the neglect of those matters more directly submitted to their charge and control.

But in respect to Newton's broad hypothesis, - as to the equal innate gravity of all matter, - may it not too strongly imply a necessity, in matter itself, to possess certain innate, inherent, or independent qualities, sufficient for its own government? Besides', if the law of force be an inherent and independent quality or principle of matter, it may be hard to understand why motion should not also be an inherent quality or principle of matter. But if the Newtonian hypothesis be not true, then what has been supposed to be different densities of the planets may be accounted for upon the principle, that uniform laws operate upon matter alike, under like circumstances; which, manifestly, could not be the case upon the Newtonian 
theory, - by which the worlds were launched on their neverending way by one original impulse, thence to be left wholly: dependent upon their equal inherent gravity for their future regulation and control, notwithstanding the infinity of disturbing forces forever operating from this supposed power of innate gravity.

But what is certainly of vast importance to the science of astronomy is, whether the force of gravity, : which retains the planets in their orbits, operates inversely as the distance, or inversely as the square of the distance, or in some other ratio.

It is quite certain that, attempting to bring the Newtonian hypothesis, of the force of attraction or gravitation, to agree or correspond with facts obtained from observation, has cost the learned much trouble in exploring for equations, by which to reconcile and harmonize the small deviations from the law, as prescribed or promulgated.

To be sure Clairaut, Euler and others protested for a long time against the law, as promulgated by Newton; for that the cause would not be adequate to the effects produced by the law, - as in case of the motion of the moon's apogee; and, for a time, the moon seemed well nigh divorced from all obligation to this earth, until Clairaut, - by a device so fraught with ingenuity that few, perhaps, will volunteer to examine its truth or error, - declared that he had reconciled the Newtonian law of gravity with the motions of the moon. This seemed to be the last great struggle. Opposition, in all quarters, appeared to give way, and philosophy seemed not only compelled, but rather inclined, to rest from opposition and strife, and to content itself with exploring for disturbing forces and equations, by which the Newtonian law of gravity might be justified, however the harmony of nature might be torn in pieces to effect the purpose. And notwithstanding many since have felt in their minds to rebel against so unjust and arbitrary a law, - as in case of Lord Monboddo and Sir Richard Phillips, as well as thousands of students who have been disciplined under this law, - yet no effectual force has ever been organized to resist it. While, on the contrary, the superstructure has, for near two centuries, been erecting upon the Newtonian foundation; in which the builders have, figuratively at least, made use of the calculus for stones, and flaxions for mortar, until their language, either spoken or written, has become so confounded, that the world at large have long since ceased to have any understanding of it whatever; while the builders, (to use a figure of M. Bailly,) like vultures over a battle-field, soar one above another by such awful flights, that one can scarce discern anoth. 
er ; while mortals, who tread the earth beneath, can scarcely discern the lowest of them.

But, instead of contending against the sufficiency of the law of gravity, as given by Newton, or endeavoring to reconcile the law thus given, with facts which utterly deny its truth, had Clairaut, Euler, and others, taken the trouble to reëxamine the evidence from which Newton deduced his supposed demonstration, they would readily have discovered where and how he committed his error, - namely, in assigning the square of the distance to the rate of force, in lieu of assigning or referring it to the amount of the fall of the body, in accordance with the law of falling bodies near the surface of the earth. This one correction in the premises might have led to a further and better consideration of the law of force or gravity, and eventually to a true development of its principles, as applied to the control of a revolving body; by which they would have discovered that the moon is entitled to just four times the force allowed to it by Newton's law of gravity, and, consequently, to twice the motion which his force would give.

But another great error in Newton's calculations (and which will be considered in its proper place) was, in supposing that the deflection of the moon from a tangent to its orbit, in a given time, would be just equal to the fall of a body from a state of rest, at the distance of the moon, in the same time.

Neither the limits nor design of this work permit me, were I disposed, to enter the lists with philosophers, (either ancient or modern,) who have theorized upon the phenomena of motion, with much curious speculation, even to the doubting of its existence. For the same reason I shall not go into the inquiry, whether, according to Newton's theory, matter, by nature, is inert, and will not move until-urged by some adequate force; and that, from the same cause,-inertia,- - when once put in motion, it would, if not counteracted or disturbed by some other force, forever continue to move in a right line, with a uniform velocity.

But, perhaps, after all, there may be something too imaginary in such a theory, to justify its being considered a self-evident proposition. For on recurring to the laws of nature, so far as we are able to investigate them, we find them so contrived as often to forbid what, - to our fancies or imaginations, - might occur; if the laws of nature were otherwise than they are. We know of no such thing in nature as that of a body moving, either by a uniform motion or in a straight line; for all matter not only moves in curves, but with constantly varying velocities. Nor is the dilemma to be avoided, by reference to a body falling toward the centre of the earth; for the motion 
is not only accelerated, but the curve which it represents in its fall has even perplexed La Place, and other great mathematicians, to describe it. Hence it might seem, that those who have assumed as a lemma, or self-evident proposition, that a uniform motion, in a straight line, is the natural state or condition of the motion of matter, have permitted their imaginations to devise quite a different method from that which the laws of nature any where permit.

Similar to the foregoing, is the case of those who fancy that the natural state of matter is a state of rest; and allege, that of the truth of this proposition, also, we have abundant proof. We know of no matter that is not constantly in motion, and that, too, in accordance with what appears to us to be eternal and immutable laws. To be sure, a stone, or other ponderous body, lying upon the surface of the earth, appears to us to be at rest; but whe does not know that it is the tendency of such a body to be in $r$ otion that causes it to remain in such apparent state of rest, ?.nd that its gravity is as much exerted upon the body where it lies, as if it were actually falling towards the earth ?

So, also, the assumption appears to have been gratuitous and wholly uncalled fur, that the heavenly bodies must necessarily have been projected, as with a view to motion, in straight lines; but that, by the power possessed by inert matter, they were wholly prevented from moving in the direction in which they were projected; and this upon the assumed principle, that the forces which produce motion in a curve are resulting forces, that is, that the motion in a curve is the result of two forces momentarily applied; or that the straight line which the body would pursue, were it not deflected from the same, arises from the inertia of the body in a state of motion, being the result of force long since expended; and that the deflecting force is constantly or momentarily operating upon the inertia of the body, as though it were in a state of rest. But, upon the principle of resulting forces, it may be hard to conceive why the motion should not be continually or momentarily increased; especially, if the circle be what many have conceived it to be, namely, a polygon consisting of an infinite number of sides; in which case, the deflecting force would be exerted by many different impulses, and, consequently, the rate of motion would be constantly changing. Nevertheless, the motion in a circle remains as uniform as in a straight line; and Lord Monboddo says, that in the days of Aristotle the line of a circle was considered as simple as that of a straight line; and in accordance with this idea, it is certain, that the only rational conclusion to be deduced from the attempts of those who would ascertain the 
periphery of a circle, by a reduction of its inscribed and circumscribed polygons, is, that the angles of the polygons, by becoming more and more obtuse, at length wholly vanish in the line of the circle; and, hence, that where a tangent touches the periphery of the circle, it forms no angle whatever with the line of the circle.

But we nowhere discover, in the mechanism of the heavens, that force is exerted by impulses; and it would seem that the composition and resolution of forces, - as taught upon the parallelogram or otherwise, - instead of teaching or inducing such notions of force and motion, as being applicable to the heavenly bodies, would have simply taught that motion, produced by impulses, is as the square root of the force applied. But how the eternal and immutable laws of nature would have been, had they been otherwise than they are, I know not ; nor is my imagination boundless enough to comprehend things so vast, and give the information to mankind; and should I attempt it, I should, probably, find myself in "endless mazes lost."

In reference to the science of astronomy, - which, from its nature, requires, perhaps more than any other science, the aid of mathematics, or rather the application of numbers in the development of its truths, - may it not be questioned, whether a greater regard should not have been had to the edification of those, whose lack of leisure and of other facilities, too often deprive them of the requisite advantages for investigating the more abstruse sciences, and which might be afforded them, were the learned inclined to stoop a little more to their necessities in that respect?

Thus the mathematical languages, if I may so speak, in which the science of astronomy is now generally taught, are become absolutely terrific to the great mass of mankind; by which means much of the science is to them but little if any better than Rosicrusian mysteries; which fact has a tendency to cause their knowledge of the science to become less, in proportion as the science is supposed to become advanced. For I will ask, what proportion of a community can be expected to obtain a knowledge of algebra, fluxions, or the calculus, or an infinity of abstruse geometrical diagrams, with the view of obtaining a knowledge of the science of astronomy, whereby they may become able to distinguish for themselves the beauty of truth from the deformity of error?

But happy, however, may it have been for some, that they were thus enabled to obscure their philosophy, even to such a degree that the learned themselves were unable to penetrate or dispel the dark fogs and mists that pervaded it. And it is 
almost to be feared, that the libraries of the present day will share the fate of the Alexandrian, from so universal an ignorance of their contents, or from our not being able to understand and appreciate them.

Neither Galileo, nor Kepler, knew aught of the differential or the integral calculus, or of fluxions; nor had Des Cartes applied algebra to astronomical investigations. Kepler made use of the then popular method, by the use of the Arabic characters; and that, too, even without the assistance of logarithms. But since the days of Kepler, I know not of a single discovery in physical astronomy which deserves the name.

May it not, then, be possible, that less rigid or thorough mathematical investigations have obtained, in consequence of the adoption of other methods than would have been used, if the popular or Arabic character had been retained in all mathematical investigations? for of the use of it, all must have some knowledge who have obtained the rudiments of an education.

And may it not even now be questioned, whether the ordinary mathematics, by popular notation, is not as susceptible of demonstrating the most abstruse problems or propositions' and of investigating as rigidly, as clearly, and with as much scrutiny, the arcana appertaining to mathematical science, as any of the substitutes extant? This method is, certainly, the only one with which the mass of mankind will probably become acquainted; and, hence, to adopt into the sciences such a method to the extent of its powers, would certainly bring science vastly more within the reach or understanding of the mass of mankind, than it is at present; and vastly more would be gained to science by the general diffusion of knowledge, than perhaps would be lost by a total abandonment of all other methods.

These being my views, I shall, in the present work, confine myself, as much as possible, to the common or popular method in mathematics; whether it be in attempting to disclose new principles in the economy or philosophy of numbers; or in applying them to the investigation of the laws of nature, or of scientific truth; making use of as few additional symbols or characters for the expression of abstract ideas, (in addition to the natural numbers, or popular notation, ) as the subject will admit.

It is not, perhaps, yet known to what extent the economy of numbers, (even by the common or popular method,) may be carried, in their development of the human mind in its upward progress; which, from being, in their simplest application, perhaps, the most abstract and simple of all our ideas, may also be extended into the most intricate, abstruse and confused. For 
although unity, or individuality, is, perhaps, as distinct a conception as the mind entertains, yet, in proportion as unities or individualities are increased or multiplied, they become more and more indistinct or incomprehensible to the mind; so that few, perhaps, have very clear conceptions of a million.

In the economy of numbers, as in other things contrived by infinite wisdom for the benefit of man, there is a fitness of things fully commensurate with the human understanding; and to a knowledge of which, to the utmost of human capability, he seems to be invited and urged by every consideration worthy of his unbounded capacity, when fully developed.

Although it is not my purpose, in this place, to treat particularly of the economy of numbers, it may, nevertheless, not be wholly unprofitable to commence with it, and refer to some of the qualities of unity or 1, and their peculiar application to the investigation of the immutable laws of nature.

Unity; then, or number 1, seems wonderfully calculated or adapted, as a numerical standard, for all quantities capable or susceptible of numerical value or admeasurement; as of bulk or magnitude, weight, space, time, force, motion, distance, \&c.; for, however great or small the quantity, it may be denoted by unity, which is at the same time susceptible of indefinite mathematical or numerical divisibility. But what peculiarly distinguishes unity from all other numerical quantities, and makes it the standard numerical quantity by which to compare all others, is, that its powers and roots neither augment nor diminish it. This, together with the constant reciprocity which exists in the elements of the heavenly bodies, as connected with the laws by which they are governed, whether it be in reference to the elements of an individual planet of a system, or of all the several planets of a "system when compared with each other, makes it of the first importance, in facilitating our operations and in arriving at satisfactory results, that unity be adopted as the standard measure of all numerical quantities or values. This being the case, by always regarding unity as the radical centre of all numerical quantities, the laws which govern the planetary world will be found to be based upon much more uniform and simple principles than they have in general been supposed to be, all harmonizing and agreeing with the Keplerian law, that the squares of the periods are as the cubes of the mean distances from the sun. Thus, in any system of bodies revolving about a central force, as in case of the primary planets about the sun, or of satellites about a primary planet, either of the revolving. bodies may be taken at the mean distance of unity or 1 , from the central force, by which to compare the elements of all the 
other revolving bodies, - their respective distances being proportioned to that the distance of which is taken at unity. And in such case, each and every element of that planet which is adopted as the standard, - or the distance of which is assumed at unity, - must also be assumed at unity, in order that the Kep. lerian law may be fulfilled.

In respect to whatever of theoretic matter I may introduce, as supposed deductions and conclusions drawn by way of analogy from known facts and laws, I shall feel no anxiety in sustaining the same, further than it shall be sustained by legitimate evidence; as in case of my theory in respect to the identity between magnetism and the attraction of gravitation by which the heavenly bodies are controlled, of which I have conceived many evidences to exist in the phenomena of nature. In support of this theory, I shall adduce the evidence arising from the different powers of attraction of the different planets, in proportion to their respective bulks or magnitudes; and in this case, I shall attempt to show that the phenomena arises from a general law, (not fortuitous) deducible from the identity of gravitation with magnetic attraction.

I shall call in the aid of the phenomena of the tides, arising from the attractive power of the moon; and shall, on this principle, endeavor to account for many of the phenomena in respect to the tides, in a very different way and manner from those commonly adopted respecting them; and show why equal tides can exist on opposite sides of the earth at the same time; why the tide-waters of broad oceans pile high upon the shores of broad continents, while they are comparatively small on the shores of the narrow parts of those continents, and still smaller in the midst of broad oceans, where only small islands exist, \&c. ; and will endeavor to account, from the same simple cause, for the reason why high tide is not as far behind the moon in the midst of broad oceans, as upon the shores of broad continents; why large inland seas produce little or no perceptible tides, \&c.

In support of the identity of gravitation and magnetism, to some extent, at least, I propose to call to my aid the nice and critical observations of Dr. Bradley, (Astronomer Royal to Geo. II, made upon some of the fixed stars, with the view of ascertaining whether they had any sensible parallax; in which he finally deduced the hypothesis of the aberration of light, which, in astronomy, has become technical in its use and signification, denoting a small apparent motion of the fixed stars, occasioned by the progressive motion of light and the earth's annual motion in its orbit. And although the hypothesis has, since his announcement of it, been elaborated into a sys- 
tem or branch of philosophy, by the aid and assistance of the higher branches of mathematics, and its supposed consequences or effects introduced into astronomical observations and calculations, I must declare my full dissent to the correctness of the conclusion or hypothesis, believing the same to be in nowise justified by the premises from which it is drawn.

In these introductory remarks I will refer to but one other subject connected with my theoretic speculation," as collateral or rather presumptive evidence in favor of that unity of purpose, and fitness of things, which all will readily ascribe to the Divine Architect of the universe. I refer to the subject of Geology, a subject which, though recent as a science, or subject of investigation, abounds with that multiplicity of facts and phenomena which has already caused theories in respect to it, to succeed each other in rather rapid succession, from the circumstance of a few newly discovered facts overturning the last formed theory.

But the Huttonian, or Volcanic theory,-that the surface of the earth is but the cooled shell of what was once an entire mass of fused, or melted matter, and that all the supposed catastrophes that have happened to the earth, in producing the appearances and phenomena on and near its surface, as also the increase of the temperature of heat as we descend into the earth, \&c., which are conceived to be the evidences of such hypothesis,-is, in the opinion of many, so deeply laid as not to be easily overturned. But, to me, this hypothesis seems altogether too fortuitous, both in its origin and consequences, to obtain credence for a moment, from one who believes creation to be sustained by immutable and uniform laws; and seems far better calculated to furnish food for the marvellous, than to lead mankind to very high notions in respect to the wisdom, power, and goodness of their Creator. It would seem also, as if there would exist no necessity for such an hypothesis; which so illy accounts for the phenomena to which it is assigned, if due regard were had, or proper consideration given, to what were, until of late, occult laws or operations of nature,- - but which, in modern times, have been deduced and drawn forth by the sagacity of man, not as unprofitable knowledge, but such as ministers to his safety; his support and his happiness. I refer to the modus operandi of the law of electricity, in the experiment by Dr. Franklin with the string of a kite; to galvanism, in the experiment by Galvani with the leg of a frog; and to electro-magnetism, and the experiments by Oersted, of no less sagacious intellect.

Those operations, or laws of nature, together with that of magnetism, (and, perhaps, the operation of light,) are, in general, 
considered by philosophers so far identical, as, at least, to be modifications of one great and universal law, the constant effect of whose silent operations, are more apparent under the form of gravitation or attraction, than under any other; and that this universal law, in all its various modifications and operations, acts as a messenger of Omnipresence, as, well in the government and control of inanimate and unorganized matter, as in that which is organized and animate, and, therefore, dependent upon final causes. We see the operation of this law or principle, in gravity or attraction, in controlling the heavenly bodies in their motions. We see its operation in magnetism or magnetic attraction, as denoted by a common magnet or loadstone; presenting that wonderful phenomenon exhibited in the polarity of a common compass needle, - accompanied by the paradoxical fact or phenomenon, that what is termed the north pole of the needle, is attracted by the south, and repelled by the north pole of another needle; which paradoxical fact 1 propose to notice hereafter. Here then we discover (in the absence of any more appropriate word by which to explain the occult operation) the magnetic fluid operating in different directions, namely, towards the opposite poles of the earth, (except in case of the variation or declination of the needle,) and this upon all parts of the surface of the earth; while at the poles of the earth, it can have but one direction, as denoted by the dip of the needle.

If, then, we find this all-pervading, all-powerful, but occult law, thus operating throughout the surface of the earth, (and analogy would indicate the same in respect to other planets, ) if we find it operating in the movement and control of the vast bodies which compose the solar system; if we find it, under other modifications or appearances, as those of electricity, galvanism, or electro-magnetism, (when subjected to the control of man, and made to subserve his purposes and curiosity,) operating by its own immutable laws upon a small scale, as that of the electric, the galvanic or electro-magnetic battery, or of the telegraph, - is it to be supposed; or presumed, that those operations are only incident to human contrivance? or, rather, on the contrary, are not those laws, or principles, designed for similar but vast and continued operations in the control of the universe, which, otherwise, must be left to fortuity or chance? For it is inconceivable, how an immutable law can be subjected to the performance of operations not in accordance with its common sphere of action.

I would ask, then, if what are termed the positive and negative fluids (for here I will use no other expression to denote them) of this all powerful agent, (under whatever modification 
it may operate, meet in concentration at the centre of the earth, whether it would not far better account for all the phenomena upon and near the surface of the earth, than does the present hypothesis of internal fires, as also for an infinite variety of other phenomena, which the present hypothesis in nowise accounts for, - as that of the independent coal formation, for example?

i. Besides, upon the theory which I propose, there would be nothing fortuitous; all would result from the operation of continued laws; there would be no apprehension that the internal fires would become extinct; or that the crust might give way, and produce catastrophes, such as the imagination alone can conceive.

As no excuse is pleaded for certain courses which I see proper to take, or pursue, in the work, hence none need be granted; - as, for instance, in case of the investigation of the law of gravity, from a consideration of the law of falling bodies; - in which case the text on which I comment, (generally taken from Maclaurin, Vince or Rees,) may not only appear to some to be too often and too liberally quoted, but the commentaries may seem to savor too much of repetition, all tending to prove one and the same thing, namely, that the force of gravity varies inversely as the distance varies. It is but few subjects, however, of which I treat, and those are fundamental and important; and the course I have pursued, I believe, has ever been ésteemed laudable, in regard to important truths. They should be kept before the people, until their importance shall be duly considered and appreciated; they should even be taught by way of inculcation : and if error is to be removed, to make room for the truth, the task is doubled. We should "try every art," like a bird that "tries to tempt her new fledged offspring to the skies;" nor be satisfied with some formal intimation, believing the world to be under obligation to hold or regard it as truth, or perish. And although we may be inclined to adore whatever human devices may have established, nevertheless, truth is too catholic in its nature to be established by convention.

It is not expected that the human race will be born prodigies, or even possessing much intuitive knowledge; nor is it rationally to be supposed, that a precocity will soon attach to human nature, whereby infancy shall be the wisest portion of our lives; and whenever such has been the case, and any have become the oracles at which we might knock, or to whom we might apply for wisdom, - we have beheld the sad reality, to use the language of Job, that " wisdom has died with them." No philosopher has yet been sagacious enough, (or has yet had opportunity,) to 
detect and seize the ethereal spark and blow it into a flame; to lay hold of the evanescent thread in the tissue, and to unravel and draw it forth, for the edification and benefit of man, as in case of some of the other great laws of nature, long hidden and occult, as those of electricity, galvanism, electromagnetism, \&c.

Nevertheless, those prodigies always seem inclined to develope or disclose their numerical results, by means of legitimate numbers; and, hence, to the mass of mankind, they are, in some respects, equally as beneficial and far more intelligible, than many who have conceived their own craniums to be the very archives of wisdom.

Since, then, mankind must plod on, - not only in the acquiring of knowledge from experience, but also from the stock which has been accumulated as the general store, - it is scarcely a mark of wisdom, or philosophy, for those who have been placed as file leaders, in the great march of human improvement, (or who have been regarded as such,) to lead off so far in advance, that their position can only be distinguished by the reflection of their names from the sky; and they hence become no guide whatever to the multitude.

But as physical astronomy ought to have its foundation based on truth, and as the involution and evolution of all the strange curves in which modern astronomy compels the planetary system to wander, are subjected, for the accuracy of their inflexure, to the curve of the circle, it is certainly desirable that the circle, in order that it may be a true standard, should be mathematically round. And in order to furnish such standard, I shall proceed to an investigation of the quadrature of the circle, prior to a direct investigation of the laws of force and motion.

In my investigation of the quadrature, (as in all of my other investigations, ) I have endeavored to render the process as simple and easy as possible, carefully avoiding any method that may not at once be made familiar to any one having the rudiments of an education. Hence I have adopted the letter $\mathrm{p}$, to express or denote generally the word polygon; and this from the circumstance that a figure at the right of the letter, may readily denote the number of sides of the polygon, whenever it may be necessary to give the number of sides. And this, together with a few denotations of polygonal quantities, expressed by letters of the alphabet, with one or two signs definitely explained, is all the analytical machinery which I propose to adopt or use. For I have yet to learn, that the beauty of truth depends upon its robes, however flaunting or party-colored they may be. 
Hence, it does not require a volume of fluxions, and the calculus, to explain the quadrature to mankind, or give them rational notions of the curve of the humble circle; for all agree, that those methods will not disclose the ratio between the diameter and circumference. Archimedes, in his sagacity, sought out the true method; but, having fallen into an error in the operation, he missed the mark, and thence sought to detect the error to the day of his death; but, having failed, the error has yet remained, and has at length been extended to a prodigious length of tail.

But had Archimedes adopted unity as the diameter of the circle, and geometrized upon lines in lieu of areas, he would at once have discovered wherein his error lay. And hence, in respect to the circle, we have a notable instance of the tenacity of mankind, in adhering to and sustaining error, (however wide from the truth,) if they imagine the law to be with them; as in the case, also, of Dr. Bradley's theory of the Progressive Motion of Light in Time, - which theory has placed the sun some eight and one-fourth minutes of time out of its apparent place, - which theory has been elaborated by fluxions and the calculus, until no one is justified in believing his own eyes; when, perhaps, the error of the hypothesis may be readily shown by the simplest diagram, and also that the Dr. drew conclusions directly the reverse of the phenomena from which he deduced them.

But an instance of this kind, not less remarkable, perhaps, than any other, is that in respect to the Newtonian law of gravity, - the demonstration of which, perhaps, many suppose to be a result obtained through the medium of fluxions, the calculus, the problem of the three bodies, or by some mystical operation of corpuscles or physical atoms upon each other, \&c., when even the Newtonian method, on which he labored for years, was so extremely simple, that any school-boy may reëxamine it, and detect the error which Newton made. And such is the ease with which all physical or mathematical truths may be demonstrated, whilst error often requires a strange and uncommon dress to hide its deformities. But

\section{"Patron or intercessor, none appeared."}

"And I looked, and there was none to help; and I wondered that there was none to uphold." 


\section{UNITY OF PURPOSE, ETC.}

\section{H A P T E R I.}

\section{On the Quadrature of the Circle.}

\section{SECTION FIRST.}

To find a rational proportion between the diameter and circumference of the circle, which might be definitely expressed in some given power, or root, of numbers, has long been a desideratum with mankind, as we learn not only from tradition and the history of geometry and mathematics, but, also, from every elementary work upon geometry and mathematics, in which we readily discover that a very great portion of all the geometric problems, propositions and demonstrations, have had in view to ascertain the true ratio between the diameter and circumference of the circle, as though it were the one thing needful in science.

Nor has this longing for a paramount truth been without its beneficial effects on science, as thereby rectilinear geometry has been developed to a vast extent; but its failure to develope the true quadrature, has elicited expressions like the following, even from modest authors, namely: "If there existed a rational proportion, - that is, a proportion to be expressed in whole numbers, - of the surface of a circle to a square surface, there would be, at the same time, a rational proportion between the diameter and the circumference. But, from geometrical reasons, no rational proportion of the diameter to the circumference is possible; it can be expressed only by approximation. 
However, the proportion thus obtained is quite as correct as is necessary for any purpose in the applied mathematics." But, however modest the above allegations are, compared with those of some authors, I must dissent, not only from what the author alleges as truths, but also from the consequence and effect which such kind of teaching is calculated to have on mankind.

Why should the author speak of a ratio expressed in whole numbers, when even the ratio between the linear measures of the square cannot be so expressed, as that between the diagonal and cireumference, but must be expressed in the second power of numbers? And even though the ratio of the diameter of the circle to its circumference cannot be definitely expressed short of the third power, - or some power higher than the second, - it will be no less a definite expression of the true ratio, than though it could be expressed in whole numbers in the first power. Nor was the author, perhaps, aware, how much more beneficial to science the true ratio would be, though it were only capable of being definitely expressed in some higher power than the first or second.

Thus the expression of the author is fallacious. But when the author alleges that, "from geometrical reasons, no rational proportion of the diameter to the circumference is possible," I think the allegation altogether too bold. to aid science in its progress ; and it is an allegation certainly uncalled for, by those who are in search of truth. It is sufficient that geometry, in the hands of those who have furnished it with its reasons, has not been able to disclose to mortals the true ratio between the diameter and circumference of the circle.

But the same author, with all his wonted modesty, has gone much further, and made use of allegations far more reprehensible, when he alleges that the ratio, obtained by a supposed approximation, is quite as accurate as is necessary for any purpose in the applied mathematics. I have yet to learn that fundamental errors are as good as the truth, let such doctrine come from what source it may; and I predict that the popular ratio will, sooner or later, be found not quite as accurate as is necessary. But the supposition is, that geometry has not only furnished reasons why no definite proportion, or ratio, can exist between the diameter and circumference of the circle, but that it has been able to direct mankind to an error so near the truth, that it is equally as good, or beneficial, as truth itself. Thus geometry itself, so full of rectitude and truth, must be bent and warped from its legitimate station, and made subservient to human orror. 
Nevertheless, the Pythagorean theorem, or 47th problem of the 1st Book of Euclid, with its infinity of mathematical applications, (as conclusive to the mind, as is the geometric demonstration from which they are deduced,) yet remains, a boon to science; thus for example,-that the diagonal and one side of the square, may have a definite ratio expressed in the second power of numbers. That, if any given surface be conceived to be enlarged or diminished, (still retaining the same form, the area or surface will be enlarged or diminished, in a duplicate ratio to that of any given line or measure of such area. That when the diameter of the circle is unity, the area is equal to onefourth of the periphery; and that the area of any circumscribing polygon of such circle is equal to one-fourth of the perimeter of such polygon, and that the area of the circumscribed square is equal to the diameter of such circle. That, if the diagonal of a square be 2 , or twice unity, the area of such square is equal to the diameter of its circumscribed circle. That, as the area of the circumscribed square of a circle is double that of the area of the inscribed square of such circle, so the area of the circumscribed circle, of a given square, is double the area of the inscribed circle of the same square, for the proportions of their respective diameters are the same; and that one-fourth of the perimeter of any regular polygon, multiplied by the diameter of the inscribed circle of such polygon, gives the area of such polygon; and that the same holds in respect to the circle.

These, together with the infinity of corollaries and consequents, which flow from legitimate geometric demonstrations, are, doubtless, sufficient to enable mankind to ascertain the true quadrature, by the aid of right reason.

Thus, a very great part of our labor, on this portion of the subject, must consist in bringing together and comparing demonstrated principles, and mathematically arranging them, in such manner as to enable us to arrive at the required result. This, perhaps, may be done, if we take proper heed to those very appropriate remarks of $M$. Legendre, in which he alleges, that " by deducing consequences from one or more propositions, we may be led back to some proposition already proved; and that an indubitable proof of their certainty is, that however we combine them together, provided only our reasoning be correct, the results we obtain are always perfectly accurate." Such language differs widely, however, from that actual abomination to science, to which the same author stoops, when he alleges, that "' the problem of the quadrature is now degraded to one of those idle questions, about which no one possessing the least tincture of 
geometrical science, will spend any portion of his time; that although the true ratio of the diameter to the circumference has not been obtained, yet, that no advantage whatever would be derived from the true ratio, over the approximate ratio, \&c.;" thus boldly alleging that error is equally beneficial with the truth. And I will here ask of science, (and perhaps the disciples of Copernicus can answer,) if any known distance from the truth can well be ascertained, if we know not where the truth lies?

The idea or conception of invoking the aid of geometry, for the purposes of a true induction into a polygonal progression, whereby the ratio between the diameter and circumference of the circle might be obtained, is of high antiquity, as such was the method adopted by Archimedes. And it is generally acknowledged, that no essential improvement has yet been made either in his process or result, otherwise than by means of certain devices for facilitating what is supposed to be a true approximation towards the truth; and it seems to have been taken for granted, that Archimedes was satisfied with his method, and the results arising therefrom. But, in my view, it is wholly unreasonable to suppose that Archimedes, after having written a treatise upon the quadrature, if he had been satisfied with the principles upon which he had proceeded, or with the results arrived at, would, at the age of seventy-five years, have been poking in the sand, with the view to catch a vision of the true principles of the quadrature. He, doubtless, perceived that his method of deducing a progression from his geometric formula, would necessarily lead to discrepancy and error, and that there existed, somewhere in his device, an assumption based upon false and erroneous premises. But we do not learn that he attempted to degrade the question of the quadrature, or to call those fools who might attempt to improve upon what he had done, (as if wisdom was to die with him,) as has been the case with some others; nor do we find that he was bold enough to deny the existence of what he did not comprehend, or to allege, as a teacher of science, that error is equally beneficial with truth.

I shall not pretend or allege, that the popular error in respect to the quadrature, arises from any defect in rectilinear geometry, as that is perfect in its legitimate sphere; nevertheless, all experience has shown that the equation of curves, as well as of the laws of force and motion operating in planetary orbits, is properly transferred to the transcendentalism of logarithms : Nor has the necessity of a logarithmic equation been overlooked in attempts to develop the true quadrature; and, indeed, the most approved method of extending what is conceived to be an ap- 
proximation, is based upon a logarithmic series. But I am constrained to allege, that in lieu of adapting a logarithmic series to a true equation of the circle, they have only adapted it to those errors incident to their supposed approximation, and which, of course, only serves to confirm the error. For they have wholly disregarded the varying capacities of polygonal measures, otherwise than that arising from the presumption that the perimetrical capacities were constantly increasing from the trigon or square, to the periphery of the circle. And consequently they have given the periphery of the circle a greater capacity than it actually possesses; for, if there be other extraordinary capacities to be consulted or considered, than those of the perimeters only, in a polygonal progression from the trigon (or from the square) to the circle, it must be manifest, that the final capacity of the perimeter of the circle, must depend upon a proper equation of the capacities of polygonal measures, throughout the whole progression. If, however, we attempt an approximation by means of a logarithmic series, it is certainly of importance that such series should be adopted as will lead to right conclusions; nor should some solitary coincidence in the application of numbers, (supposed to have some analogy,) be sufficient to establish the correctness of such logarithmic series; as may, perhaps, have been the case in reference to a series carried on by an alternate division by the odd numbers, upon the supposition that they are equally applicable to the deflection of a revolving body from a tangent to its orbit, as to the rectilinear fall of bodies near the earth; and, consequently, that they had a like application to the curve of the circle; and thus, trusting to fallacious appearances or unfounded analogies, the truth has been permitted to suffer. For, notwithstanding the law of the rectilinear fall of bodies near the earth has so beautiful and simple a numerical expression, by which law the sum of the spaces passed over in as many equal moments from the commencement of the fall, is the square of the whole number of moments, (or times;) and also, by which the square of the sum of all the moments (or times) will be the sum of all the odd numbers in their order, up to the number of moments, (or times,) yet, I am quite apprehensive that those beautiful coincidences have been intruded into those investigations with which they have no particular concern, and have induced conclusions highly detrimental to science. It is not my intention, however, to reject legitimate evidence, in whatever form it may be offered; whether it be direct and positive, or only presumptive and corroborative, - as by the reductio ad absurdum; or such as shall arise from the discrepancies and 
errors of the popular methods; and I shall even attempt to induce assent to the truth of my conclusion, from the beauty and harmony of its analogies and applications, and its coincidences with all the great laws of nature, which are the subjects of numerical or mathematical calculation. For, to determine the true measures of the circle, and to know from indubitable proof that such determination is right, is, perhaps, all that can rationally be required; nor are we to be debarred from any legitimate evidence that may arise to sustain us in our knowledge of the truth.

My purpose is, to prove to the satisfaction of the world, that the circumference of the circle, whose diameter is unity or 1 , is 3.1748020 , or the third or cube root of 32 , and hence that the area, or one fourth of the circumference is .7937005 , or the cube root of .5 , in lieu of the popular series, - .78539816339, \&c., \&c. ; thus making the cube or third power of the diameter of the circle, twice the third power of one fourth of the circumference; and, consequently, that the ratio of the diameter to the circumference is commensurable in the third powers and roots of definite numbers.

It is agreed that the surfaces of solids are as their bulks : That the product of one sixth of the surface of any regular solid by the diameter of its inscribed sphere, gives the bulk or contents: That the bulks of solids increase or decrease in a triplicate ratio, to that of any given linear measure of the solid: That the surface of a solid increases or decreases by a duplicate ratio to that of its linear measure: And also, that the ratio of the increase of surface to that of bulk, is as that of the diameter of the orbits to the periods of the planets.

It has been well demonstrated, that the bulk of the sphere is two thirds that of its circumscribing cylinder; hence, by my determination, the bulk of the prime sphere, or sphere whose diameter is unity, is the third root of the fraction .148148, ad infinitum; and the third power of its surface is 32 . These proportions between the sphere, cylinder and cube certainly present many beautiful coincidences and reciprocities, not only in the economy of numbers, but in the perfect adaptation and fitness of things in respect to surfaces and solids, or their rational admeasurement, which was anciently thought so desirable for the furtherance of science, as to be earnestly attended to. Nevertheless, the beauty, harmony and reciprocity in the application, is but presumptive evidence, and is not to be considered sufficient, for satisfying.the abstract geometer or mathematician,- - which is to be done. But as it is my intention to be tried 
by my peers, I shall address myself to them, in support of my cause, in language as intelligible to all as I am capable of using, or as circumstances will admit, scrupulously avoiding those methods of investigation which, to a great portion of mankind, are dark and mysterious, and absolutely repulsive and terrific, like the methods by fluxions and the calculus. For it is my intention to test the question whether the great mass of mankind are capable of judging for themselves in respect to physical science, or whether they are forever to depend upon the few profoundly learned, for an implicit faith instead of actual knowledge. And this being my purpose, perhaps none will expect me to give heed to those methods of investigation which have been so little understood, save by the profoundly learned; and which, although carried to such an awful height as to be beyond the ken of mortals in general, have never, as yet, perhaps, disclosed or developed a single physical truth worthy of science. The method which I have adopted for comparing polygonal quantities, with a view to some unity of purpose, varies somewhat, in many respects, from the popular methods; but nevertheless, retains a sufficient similarity to enable us to make all proper comparisons between the methods and results arrived at. And as it is my intention to avail myself, in behalf of my views, of the discrepancies and errors incident to the popular methods and results; and to show that they are wholly inadequate to equate the varying capacities of polygonal measures, it will be my endeavor, so far as I may refer to the popular methods, and the results arising therefrom, with a view to a just comparison, faithfully to translate the same as nearly to some unity of purpose, as the idiom or nature of the case will admit; and to give full credit to the authors, in the use of the general appellation of popular methods or popular determination.

It is often alleged by authors, (and perhaps with too much emphasis or technicality,) that the circle, or any given polygon, is properly the area or surface; but that, in the common acceptation, the periphery, or perimeter alone is called the circle, or polygon ; and I must confess, that, for most purposes, I am inclined to the common acceptation; for the reason that it is the linear quantities which must determine the numeral amount of area or surface, and not the converse. The ratio between a line which forms a perfect circle, and its greatest rectilinear extension, (called the diameter,) is what has been so long and so anxiously sought; and if such ratio be obtained, we shall have no difficulty in assigning the ratio of the area to that of the square, whose area is called unity; whether such area of the square be merely conventional, as being most convenient, as a standard, or whether 
it is founded in the fitness of things; and which, from necessity, has induced its adoption as the standard. And it certainly is more proper to speak of the varying capacities of linear measures for bounding area or surface, than to speak of the capacities of the areas or surfaces themselves.

We very properly speak of the circle, the square, the octagon, \&c., without regard to their numerical dimensions; and such should always be the technical consideration of all regular polygons; namely, that a regular polygon, having any given number of sides, is properly one and the same polygon, whatever may be its numerical dimensions. But as a polygon must often be compared with the same polygon, possessing different numerical dimensions, it will often be the most convenient mode of comparison, to consider the same polygons, when expressed by different numerical dimensions, as being like polygons; that is, one is like the other. Thus, in the case of the prime circle, or circle whose diameter is 1 , the same polygon (of whatever number of sides) may be inscribed within, or circumscribed about the circle; and the inscribed polygon may be said to be like, or the same polygon with, the one which circumscribes the circle. And the importance of considering the identity or sameness of a regular polygon to depend upon the number of its sides, and not at all upon its numerical dimensions, in our investigations of the quadrature, is, perhaps, much greater than might generally be supposed; and hence I shall endeavor to enforce the necessity of such consideration in those places where a different consideration must necessarily lead to discrepancy and error. Thus, calling the circle whose diameter is unity, the prime circle, any circumscribing polygon with its like inscribed polygon, forms a prime polygon, and is properly to be considered as one and the same polygon, possessing different numerical dimensions; the circumscribed, being called the major polygon, and the inscribed being called the minor polygon. And in what numerical point or position the perimeters of a major and minor polygon shall actually coincide, and thereby form the periphery of the prime circle, is the question to be determined. That such periphery exists, is a self-evident proposition; nor is it in any way material, whether we conceive it to be composed of the perfect coincidence of a major and minor perimeter, or whether we conceive it to lie between the utmost major and minor perimeters; it is sufficient for us that it is but a boundary, the thickness of which we can no more comprehend than that of the infinite divisibility of matter. Nevertheless, as we have all along conceived the major and minor perimeters to be progressing towards the periphery of the circle, perhaps our 
utmost conception must be, that the periphery of the prime circle lies between the perimeters of the utmost major and minor polygons. And hence the utmost of our researches in respect to the quadrature, can extend no further than that of finding the utmost meaus, (either equal or proportional,) between two approximating quantities or numeral positions. This, however, is quite sufficient ; for geometric points and lines, having no thickness, the utmost mean between two approximating points or lines is but the actual coincidence of those points or lines; and all. must necessarily lie in, and be expressed by the same root or power. It may, nevertheless, in the course of the work, often be more in accordance with our habits of thinking, to conceive certain coincidences to operate as a merger or extinguishment of a part of the coinciding quantities, as in the case of diametric quantities, for instance; for whilst each polygon of sides is conceived to have two diametric quantities, (namely, the diameters of its inscribed and circumscribed circles,) the circle is generally conceived to have but one diametric quantity.

Nevertheless, it may often be necessary, by way of comparison and explanation, to consider the circle in the nature of a polygon of sides, and possessing like quantities as that of an inscribed and circumscribed diameter; and in case the prime circle is to be considered as the utmost polygon, partaking, as well of the properties of the minor as of the major polygons, it may also, for the purposes of comparison, be conceived to have a major and minor perimeter, and a major and minor area; in which case, the major area will be numerically equal to one fourth of the major perimeter, and one fourth of the minor perimeter will be conceived to be equal to a minor area of twice the number of sides; for such is numerically the utmost of our conception in respect to a minor perimeter and its accompanying area. And hence, so long as we retain the conception of a minor perimeter, so long we retain the conception of a minor area of twice the sides.

Now to obviate the difficulty in obtaining a satisfactory result to the mind, arising from such inevitable conceptions, as well as to determine the numerical points or position in which the major and minor perimeter of a prime polygon actually coincide and form the periphery of the prime circle, it will be necessary, lest we mistake the ratio of progression of the minor perimeter to that of the major, that we so prepare our work as to enable us measurably to comprehend the end from the beginning, by the adoption of such a course as will furnish a train of such absolute and conclusive coincidences of the ratios of progression of polygonal quantities, as inevitably direct to, and conclusively 
determine the numerical point or position in which the major and minor perimeters of the utmost prime polygon must actually coincide. And as the numeral value of the minor perimeter of any prime polygon, is, to the major perimeter, as the diameter of the inscribed circle of a like polygon is to the diameter of the circumscribed circle of such polygon, I have sought to obtain such trains of absolute coincidences, (and consequently the coincidence of the major and minor perimeter of the utmost prime polygon,) by a comparison of the class of prime polygons with that class of polygons whose respective areas are 0.5 , or half of unity, which class of polygons, in contradistinction from the prime polygons, I have thought proper to denominate primal polygons; and the four denotations by means of letters of the alphabet, by which such comparison is mostly made, I shall call prime denotations; and inasmuch as a full comparison must necessarily extend to polygons of different numerical dimensions, from those of the prime and primal, other denotations may be appended, denominated auxiliary denotations; all of which will be sufficiently explained for use, in the appropriate place.

Perhaps it will not be inappropriate here, to give some introductory hints at what will appear far more conspicuous in the work, namely: the admirable adaptation of the prime and primal polygons to an investigation of the quadrature; not only from the great number of actual coincidences so readily obtained in the ratios of progression of polygonal quantities, which so satisfactorily dictate the numeral position of the final coincidences sought; but from the manner in which those progressive ratios unfold to us the varied and revolving capacities of polygonal measures, while performing the quadrant of an orbit, (or, if we please, the two octants of the quadrant,) thereby constantly suggesting both the necessity and the manner of equating those revolving capacities.

Thus the diameter of the prime circle is the inscribed diameter of all major polygons, and the circumscribed diameter of all minor polygons ; making unity an important point in this particular, but mainly from the fact that the prime square possesses, in and of itself, important coincidences situated in unity, thereby making the square the proper polygonal standard. Thus the diameter of the inscribed circle of the square, and one fourth of the perimeter of the same square, are always numerally equal; and when situated in unity, each is necessarily equal to the area; each of which is then numerically equal to the diameter of the circumscribing circle of the primal square, or square whose area is half of unity, which circumstance becomes important in our investigations ; while one fourth of the perimeter of the minor 
square is equal to the diameter of the inscribed circle of the primal square, and is also equal to the area of the minor octagon; -thus making the square (namely, the polygon denoting unity amongst the polygons) emphatically the standard from which ratio and proportion are properly conceived to emanate, and with which they must of necessity be compared. But in all prime polygons below the square, (namely, of more sides than the square,) which we are able specifically to investigate, one fourth of the perimeter is less than the diameter of the circumscribing circle of a like primal polygon; and by the popular methods this variation is increased in the whole distance from the square to the circle; thereby making the capacity of the perimeter of the circle too great, and that of the diameter too small.

Now this variance between one fourth of the perimeter of a major polygon and the diameter of the circumscribing circle of a like primal polygon, which is proportionally the same between one fourth of the perimeter of a minor polygon and the diameter of the inscribed circle of a like primal polygon, (which variance is nothing in the square and circle, and is at its maximum in the octagon,) is always proportionally equal to twice its equation; and to denote such equation, a sign or symbol will be adopted and explained. Again, a comparison of the prime and primal polygons is well calculated to disclose certain facts and phenomena in polygonal economy, which the popular methods seem not to have heeded or to have made any account of,-and among others, the transposition of polygonal quantities in unity, or in the prime square, while passing from the trigon to polygons situated below the square,- -which phenomenon certainly deserves consideration, and will receive it, by special reference, in due time and in the proper place.

And it is possible that a notice and consideration of this transposition of polygonal quantities in unity, or the prime square, mât have suggested some defect in the popular mode of approximating by means of a logarithmic series; that is, the method of obtaining constant dividends and divisors, and a disregard of such transposition, may have afforded some of those geometrical reasons which are said to exist, why " no rational proportion of the diameter to the circumference of the circle is possible." This transposition of polygonal quantities in the major square, is necessarily such, that, for the obtaining of one fourth of the major octagon, the dividend and divisor may always be obtained from the same nominal terms of the square, as from the trigon, in deducing one fourth of the perimeter of the major hexagon; but as in any polygon below the square, the relative situations of the terms are transposed from what they are 
in the trigon, it may, at least, be questioned, whether there should not be some transposition of terms in polygons below the square, from which the requisite dividends and divisors should be obtained. Nevertheless, such transposition has not been heeded in the popular methods. It will, however, be quite manifest, that dividends and divisors obtained from any given polygon below the square, for deducing one fourth of the circumference of a major polygon of twice the sides, may be obtained of like nominal quantities of the square for deducing one fourth of the perimeter of the major octagon; but not from the trigon, in deducing one fourth of the perimeter of the major hexagon. The consequence then becomes inevitable, that if we proceed to obtain our constant dividends and divisors from nominal terms, the same as or like those we use in obtaining one fourth of the perimeter of the major hexagon and octagon, the divisor becomes too large for the dividend, and consequently the quotient will be too small; and the error will continue to increase, by infinitesimal quantities, to the final determination, producing some strange discrepancies near the final determination; - as that of forming premature coincidences and again breaking up and dispersing them; while other quantities, equally entitled to coincidence, are not permitted at all to coincide; and in the final result, those quantities which, from their ratios of progression, seemed all along destined to form one general coincidence in the circle, are scattered into various and diverse coincidences; and not a single term or polygonal quantity is permitted to remain in that numeral point or position which seemed destined, from every law of polygonal progression, to be the point of coincidence for all the progressive quantities. Thus one fourth of the periphery of the prime circle will be .7853981 , \&c., and the diameter of the circle whose area is 0.5 , will necessarily be expressed by .797892 , \&c., and the three points of equation which should have directed to the true place, are caused to coincide in .791620 ; namely : .0020s0 below the true place.

Because the product of one fourth of the perimeter of any given polygon $X$ the diameter of the inscribed circle of such polygon gives the area of such polygon, consequently, for the same cause, the area and one fourth of the perimeter of any given polygon, the diameter of whose inscribed circle is unity, are numerically equal; and hence it is equally well, and vastly more convenient in our investigations, to adopt one fourth of the perimeter as the standard circumferential measure, in lieu of the entire perimeter.

I shall plead no excuse for the desultory manner in which I 
shall treat the subject of the quadrature; it being my design solely to furnish the evidence, both in quantity and quality, to satisfy the candid inquirer of the correctness of my solution of this important problem. Hence, after setting forth an epitome of the prime table, as a table of reference, and giving some explanation of the few signs, characters, and denotations used in the work, I shall proceed to present my evidence in such a way and manner as I may conceive will elicit the most thorough and thoughtful investigation from the inquirer. And conceiving the whole subject to be wrapt up in consequents or corollaries, flowing directly from well demonstrated principles which need not to be repeated, the bare suggestion of which, by way of allegation, being, in most cases, a sufficient reference to the demonstrated principles from which they emanate or flow, at least by the aid of a little reflection, and that correct reasoning of which Legendre speaks, - I shall not, in general, do more than to allege such demonstrated truths, and such consequences as they inevitably dictate. And in this respect, I may sometimes become quite prolific in trains of such correlative matter as I may conceive will throw light upon the subject; for it is my intention to furnish indubitable proof of the correctness of my determination, by showing " that however we combine our evidence, provided only our reasoning be correct, the results we obtain will always be perfectly accurate." The limits, however, assigned to the work, will admit but a very small portion of the evidence which I have examined in favor of my determination; nor am I confident that the evidence which I shall offer, will be a judicious selection from that which I have examined.

I am aware of the ordeal which the popular determination of the quadrature of the circle has passed; and of the fortified position in which it now rests, as in the citadel of the world's last hope; and that, were it not for the eternity and immutability of the principles of ratio and proportion, the popular error must, long ere this, have been converted into a mathematical truth. And it having been thoroughly tested by fluxions and the calculus, (those methods which appear to me far better calculated for the equation of human errors, than for disclosing mathematical truths,) the learned have in general, become satisfied that the force of nature can no further go; and hence, the question is sure to be put to the modest inquirer, in respect to a true solution of the quadrature, "whether he has examined the subject by means of the fluxions and the calculus;" and still we find it alleged in Rees' Cyclopædia that the hopes of mathematicians, which were revived in respect to a true solution of the quadrature, upon the invention of fluxions and the calculus, 
were, at length, abandoned in despair, after exhausting every possible device by means of those methods, but without success; and hence a true solution is now declared to be impracticable; and geometry is charged with having furnished reasons which justify such declarations; but I have certainly understood legitimate geometry quite differently. But the problem of the quadrature ought not to be settled by convention, although I am aware that important questions in respect to science have too often been settled in that way; as in case of the theory of universal gravity; and in case of the laws by which gravity operates in respect to distance.

If, however, the popular determination of the quadrature, and also the Newtonian theory and law of gravity, are forever to remain as the world's best hope in respect to a sure foundation upon which to base their scientific researches, then, to be sure, astronomers must have an onerous task to perform, in which one equation only serves to beget other supposed variances and aberrations, and so on, ad infinitum; in which case, the toils and labors of astronomers will become perplexing, and, like the labors of Sysyphus, never-ending.

As a matter of reference, I have thought proper to close this section with an epitome of two tables; the first, or prime table, being formed by constantly multiplying unity, and each successive product obtained, by the sixth root of .2 in ascending, or by the sixth root of .5 in descending. Hence, if the natural numbers are made exponents to the terms, both in ascending and in descending, any two terms denoted by the same number, will be reciprocals of each other; the term denoted by 2 ., in the descending column, would be the same which I have so constantly denoted by $\underset{2}{2}$ in the course of the work, and is, according to my idea, the area of the circle whose diameter is unity. And in accordance with such conclusion, if the diameter of the sphere be taken in any term of the table, or if the diameter of the circle be taken in any term of the table, the third power or cube of the surface of such sphere, or the third power or cube of the circumference of such sphere, or of such circle, will be expressed in terms of the table, if the terms are sufficiently extended, as they may be at pleasure. And the third power or cube of the surface of the circumscribing cylinder of such sphere, may be found expressed in terms of the second table, in case the terms be sufficiently extended. 


\begin{tabular}{|c|c|c|}
\hline \multicolumn{2}{|c|}{ PRIME TABLE. } & SECOND TABLE. \\
\hline & 1. & .16666 \\
\hline .890898 & 1.122462 & 4.762203 \\
\hline .793700 & 1.259921 & 3.779763 \\
\hline .707106 & 1.414213 & .3333 \\
\hline .629960 & 1.587401 & 2.381101 \\
\hline 561231 & 1.781786 & 1.889881 \\
\hline .5 & 2. & .666 \\
\hline .445449 & 2.244924 & 1.190550 \\
\hline .396850 & 2.519840 & 1.058265 \\
\hline .353553 & 2.828426 & $1.333 \quad .75$ \\
\hline .314980 & 3.174802 & $\therefore .595274$ \\
\hline .280615 & 3.563572 & .472467 \\
\hline .25 & & .375 \\
\hline .222724 & 4.489848 & $3.359777 \quad .297637$ \\
\hline .198425 & 5.039684 & 4.233062 \\
\hline .176776 & 5.6568552 & $5.333 \quad 1875$ \\
\hline .157490 & 6.349602 & 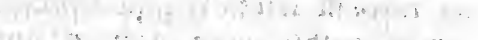 \\
\hline .140307 & 7.127144 & 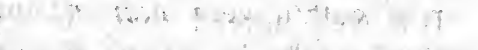 \\
\hline .125 & & $\therefore$. . is ien $=$ \\
\hline .111361 & 8.979696 & 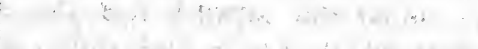 \\
\hline .099212 & 10.079360 & 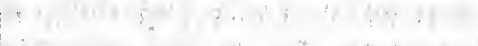 \\
\hline .088388 & 11.313704 & . 14 \\
\hline .078764 & 12.699 & 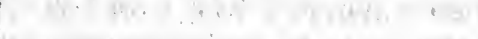 \\
\hline .070153 & 14.254288 & ete \\
\hline .0625 & 16. & \\
\hline .055680 & 17.959392 & 2. \\
\hline .049606 & 20.158720 & 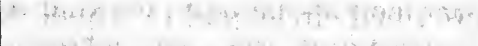 \\
\hline .044194 & 22.627408 & $3 x$ \\
\hline .039373 & 25.398416 & in $\quad \cdots$ \\
\hline .035076 & 28.5085 & se \\
\hline .03125 & 32. & \\
\hline
\end{tabular}

It will be seen that in the second table, the increase and decrease from 6, (or surface of the prime cube,) and its reciprocal, is by a duplicate ratio to that in the prime table. If the diameter and surface of a sphere be found in the prime table, its bulk will be found in the second table, in case the tables be extended so as to give the required terms.

So if the diameter and bulk of a cylinder be contained in the prime table, its surface will be contained in a term of the second table, \&c.

The above tables are only intended as examples, as they may be extended at pleasure; similar tables may also be formed, applicable to the measures of the circle. 


\section{SECTION SECOND.}

Eternal wisdom has so contrived and adapted the economy of numbers to the human capacity and the fitness of things, in all those physical operations which are subject to ratio and proportion, or to a numerical value, that it would seem wholly unnecessary for us to be groping in darkness in respect to those things, if we but take heed to that unity of purpose in which they appear to have been contrived.

But as my purpose now is to treat of polygonal economy, as applicable to the economy of numbers, I will at once suggest a fitness of things which has placed all proper investigation, in respect to polygonal progression and the quadrature of the circle, within very narrow limits, and which is, therefore, not incomprehensible to any inquirer.

It is well agreed that the square, the diameter of whose inscribed circle is assumed at unity, properly denotes unity, amongst the regular polygons, (and it is only of regular polygons which I treat,) and that the right angle is properly termed the angle of unity amongst angles; and as all ratio and proportion emanates from, and has a direct reference to or connection with unity, hence the square, which denotes unity in so many respects, - namely, the diameter of whose inscribed circle is 1 ., whose area is 1., and whose side is equal to the diameter of its inscribed circle, and in which the diameter of its inscribed circle is equal to the diameter of the circumscribed circle of the square, whose area is .5 , or half of unity, - is admirably calculated for a standard, by which to compare the quantities of the inscribed and circumscribed polygons of the circle, whose diameter is assumed at unity.

Hence, in my direct investigation of the quadrature, by the aid of symbols or denotations, I shall confine the subject to two classes of polygons, called prime and primal; the prime class consisting of the inscribed and circumscribed polygons of the circle, whose diameter is 1 . - the inscribed being called minor polygons, and the circumscribed being called major polygons; - the other class being that whose respective areas are .5 , or half of unity, which polygons of the last class I shall call primal polygons.

In the comparison of the proper quantities of these two classes, the proper quantities of a primal polygon is its inscribed 
and circumscribed diameters; namely, the diameter of its circumscribed circle, which will be denoted by $\mathrm{B}$; and the diameter of its inscribed circle, which will be denoted by $D$.

The proper quantity of a minor polygon is one fourth of its perimeter, which will be denoted by $\mathrm{C}$; and the proper quantity of a major polygon is also one fourth of its perimeter, which will be denoted by $M$.

These four letters are the prime denotations for investigating the quadrature; and should other letters or denotations be added, for the purpose of exemplification, they are to be esteemed as only secondary or auxiliary.

It will be perceived, that in the prime denotations for investigating the quadrature, I have scrupulously avoided any denotation for area; and this I have done in accordance with what I have before stated, namely, that linear quantities are the only quantities with which we have anything to do, in the investigation of the quadrature. The only question to be solved, the only mystery in the case, - is that of the ratio between diameter and circumference, wholly regardless of area, otherwise than as a consequent, depending on the ratio between the lines which measure or bound area. Hence, if we obtain the true ratio between the diameter and circumference, there will be no question in respect to the true assignment of area.

The circle, then, or any regular polygon, is properly its diametric and circumferential quantities, and not its area.

Again : that lines are the proper measures of surfaces or areas, and not areas of lines, is too manifest to require comment, notwithstanding the popular attempts to obtain the quadrature seem rather adverse to my notions; nevertheless, the ill success attending that method, has not proved the legitimacy of the method. Legitimate geometry does not geometrize upon areas, but upon lines.

Geometry, however, seeks for three or more lines, from which to deduce ratio and proportion; but it never seeks to proportion lines by means of areas, by a disregard of those lines.

Thus the business of legitimate geometry is, to proportion lines to each other; and in this it does not at all regard the varied and varying capacities of polygonal perimeters for circumscribing area. Nevertheless, whatever may be the varied capacities of polygonal perimeters for circumscribing area, in proportion to their respective diametric quantities, or however different that of the octagon, or of the polygon of sixteen sides, may be from that of the square, we shall find that what may properly be termed the capacity of the perimeter, in proportion 
to the diametric quantity, is the same in the circle as in the square.

Now it has not wholly escaped the ken of mathematicians and geometers, that the capacity of the perimeter of the octagon, for circumscribing area, is greater, in proportion to its two diametric quantities, than that of any other polygon; nevertheless, no advantage or benefit seems to have been taken of the fact, although it is of such vast importance in a consideration of the quadrature; for, while attempting a geometrical induction into a true polygonal progression, the method has been to geometrize upon areas in lieu of lines, thus losing all benefits to be derived from the varying capacities of polygonal measures.

But areas need not be even named or known, in a polygonal progression, except in the primal class, where they silently keep station in .5.

Such, then, being the case in respect to the capacities of polygonal measures for bounding area, geometry will readily dictate a polygonal progression from the major octagon to the prime circle, on true principles of ratio and proportion, and in a way and manner that cannot well be misunderstood; nor need we resort to other diagrams than such as are to be found in Legendre; and other works on geometry. It may be well here, however, to remark, that the areas of polygons are, properly speaking, proportioned to the diameters of their circumscribed circles.

I will now proceed to set forth the signs, characters and denotations to be used or employed, as a kind of analytical machinery, in deducing the quadrature, with the requisite explanations for reading the same, - those denoting multiplication, division, equality and proportion being used in the common; or popular manner.

Numerical, or numeral quantity, may properly be conceived to be that which is contained between 0 and some given numeral point, (or position, ) as that of .5 , or the square of .5 , for example ; or, numeral quantity may be contained between two points, (or positions, expressed or understood ; as that contained between .5 and 1 . for example; and such will be the technical meaning given to the word point, or points, when referring to boundaries of numerical quantities. Thus, we may speak of a root or power of a given point, or that a given point is a mean between two other given points, \&c.

The word mean, when used to denote a point between two other points, when not represented to be an equal mean, is always to be understood as denoting proportional quantity; thus 2 . is a 
mean between 4 . and 1 .; so 2 . is the farther of two means from 8. to 1., or the farther of three means from 16. to 1 ., \&c.

${ }_{1}^{1}$ denotes the first term of the prime table below unity; 2 the second term, and so on.

This sign, with a figure under it, is used to denote any given number of terms of the prime table, at which one given point may be situated either above or below another given point ; thus, for example, 2 . is $\widehat{6}$ above 1 ., \&c.

$p$ denotes, generally, a polygon, and may be read polygon.

$b$ denotes, generally, the diameter of the circumscribed circle of any given polygon, except when the area is .5; in which case it will be denoted by $B$.

$d$ denotes, generally; the diameter of the inscribed circle of any given polygon, except when the area is .5 ; in which case it will be denoted by $\mathrm{D}$.

$m$ is used to denote, generally; one fourth of the perimeter of a given polygon, except in case of a major polygon, namely, when the diameter of the inscribed circle is $1 . ;$ in which case it will be denoted by $\mathrm{M}$.

A figure placed at the right of $p$, or of any letter denoting polygonal quantity, signifies or denotes the number of sides of the given polygon; thus $p 3$ denotes the trigon, $p 4$ the square, and so on. So $b 8$ or B 8 denotes the octagon, \&c.

$\mathrm{V}$ is used to denote one side of a major polygon.

$\mathrm{W}$ is used to denote one side of a minor polygon; and a figure at the side of $\mathrm{V}$ or $\mathrm{W}$, denotes the number of sides of the given polygon:

What I shall term a series of polygons, is one commencing with the square or with a polygon, the number of whose sides is an odd number, and proceeding ad infinitum, by a constant duplication of the number of sides.

The term polygronal progression, is used to denote the order in which the prime denotations progress through the major and minor polygons, from the square to the circle.

A may be used to denote the area of a minor polygon.

I will now proceed to set forth a train of facts, which, in the course of the work, may become important; the truth of them has been so often and so well demonstrated, and they are in and of themselves so manifest, - as may readily be proved by any inquirer, - that it would be supererogation to do more than to name or allege them; and in doing this I shall not attempt method, but shall proceed in a very desultory manner, premising, however, that the point denoted by 2 , (which is the cube root of .5, ) so constantly occurs as a mean between quantities denoted by two polygonal denotations, which become propor- 
tional extremes to 2 , that I have thought proper to use the word adverse, in reference to such extremes; as that they are adverse, or one is the adverse of the other.

And first in the train of facts, I will speak of certain coincidences and reciprocities, whereby we shall find that we need not wander a great way from unity in our investigations of the quadrature.

It is understood that the area of any given major $p$ is $=\mathrm{M}$ of the same $p$; also that $\mathrm{C}$ of any given minor $p$ is $=$ the area of a minor $p$ of twice the sides.

The reciprocal of $\mathrm{M}$ of any given $p$ is $=d$ of a like $p$, whose $m$ is 1. ; and the reciprocal of $\mathrm{C}$ of any $p$ is $=b$ of a like $p$, whose $m$ is 1. ; and the reciprocal of the area of any minor $p$ is $=b$ of a $p$ of half the sides, whose $m$ is 1 .

When $d$ of any $p$ is the reciprocal of $\mathrm{M}$ of a like $p$, the area of such $p$ is $=d$; and when $b$ of any $p$ is the reciprocal of the area of a like minor $p$, the area of such $p$ is $=b$.

Whenever a $p$ is placed in connection with, or is compared with a $p$ of half the sides, it must always be understood to be a $p$ of twice the sides of another $p$; thus neither the $p 4$, nor any $p$ of an odd number of sides, can have a $p$ of half the sides.

Thus we say $b$ of any $p$ whose area is $=b$, is a mean between $b$ of a $p$ of half the sides, whose area is $=b$, and $d$ of such $p$ of half the sides, whose area is $=$ such $d$; hence, such will continue in a series, from the $p 8$ to the circle.

So $\mathrm{B}$ of any $p$ is a mean between $\mathrm{B}$ and $\mathrm{D}$ of a $p$ of half the sides; which order of progression will also continue from the $p 8$ to the circle.

Because $d$, when $m$ is $1 .$, is the reciprocal of $M$, hence, when $\mathrm{M}$ is in $2, d$ will be the cube root of 2 , or $\frac{7}{4}$ above $\mathrm{M}$. So also, if $\mathrm{C}$ be in $2, b$ will be the cube root of 2 , or $\overparen{4}$ above $\mathrm{C}$.

But 2 will be found to be an extremely important point, in respect to polygonal progression by means of the prime denotations, as also of auxiliary denotations, however they be multiplied or increased in numbers; and as well in accordance with the popular method as with my own, 2 is the farther of two means from $M$ to $D$; hence, when $M$ is in $2, D$ is also. So 2 is the farther of two means from $\mathrm{C}$, to $\mathrm{B}$ of twice the sides; so that when $\mathrm{C}$ is in $2, \mathrm{~B}$ of twice the sides is also.

This, then, presents a consequence, which, perhaps, had better be noticed here, namely, if $\mathrm{M}$ and $\mathrm{C}$ coincide in 2 , thereby forming $M$ of the prime circle, $B$ and $D$ must necessarily coincide in 2 , thereby forming $D$ or $B$ of the circle; in which case, $B$ of twice the sides could not there exist in reality. And it will be 
fully shown that if prime or auxiliary denotations, however numerous they may become, have their final place in 2 ; that all the denotations (except those which coincide above 1.,) will also coincide and have their final place in 2 ; in which point all denotations coinciding below 1 . will be merged, or they will be extinguished in the denotations of the circle.

When $\mathrm{M}$ and $\mathrm{D}$ are in $\underset{2}{2}$, half the square of $d$, when $m$ is 1 , is also in $\underset{2}{2}$. So when $\mathrm{C}$ is in $\underset{2}{2}$, half the square of $b$, when $m$ is 1 , is also in 2 . And when $m$ of any given $p$ is $=\mathrm{B}$ of a like $p$, the area of such $p$ is half the square of $b$ of a like $p$, whose $m$ is 1 . So, also, when $m$ of any given $p$ is $=\mathrm{D}$ of a like $p$, the area of such $p$ is = half the square of $d$ of a like $p$ whose $m$ is 1 .

And it may be here remarked, that the easiest and simplest method of obtaining M 8 from terms of the $p 4$, would be the same by the popular method, as by my own, - namely, by dividing the product of $\mathrm{M} 4$ by $\mathrm{C} 4$, by half the square of $d 8$ when $m 8$ is 1 . But it is quite certain, that such process will not avail any farther than that of finding $M 8,-$ as it is determined or shown to be otherwise, even by the popular method and determination; for in the popular determination, $\mathrm{B}$ by $\mathrm{D} \div$ half the square of $b$, when $m$ is 1 , gives $\mathrm{M}$, - that is, according to the final coincidences deduced by the popular method.

Hence, a method which will determine $M 8$, is not sure to determine $\mathbf{M}$ of any $p$ farther advanced in the series.

Any given linear measure of a given $p$, is always a mean between the area of such $p$, (whatever may be its dimensions,) and the point in which the area and a like linear measure of a like $p$, are $=$; thus $m$ of any given $p$, is a mean between the area of such $p$, and $\mathrm{M}$ of a like $p$, for the reason that if area be conceived to be either increased or diminished, (still retaining the same form, area will flow by a duplicate ratio to that of any linear measure of such area.

In any given $p, \mathrm{C}$ is to $\mathrm{D}$ as $\mathrm{M}$ is to $\mathrm{B},-$ hence, $\mathrm{C}$ is to $\mathrm{M}$ as $\mathrm{D}$ is to $\mathrm{B}$. But in the $p 3$ the relative positions of $\mathrm{C}$ and $\mathrm{D}$, and of $M$ and $B$ are different from what they are in any given $p$ below the $p 4$, or between the $p 4$ and circle. Thus, in the $p$ $3, \mathrm{M}$ is above $\mathrm{B}$, and $\mathrm{C}$ is above $\mathrm{D},-$ but in any $p$ between the $p 4$ and circle, $\mathrm{B}$ is above $\mathrm{M}$, and $\mathrm{D}$ above $\mathrm{C}$; while in the $p 4$, $\mathrm{B}$ and $\mathrm{M}$ are $=$, and $\mathrm{D}$ and $\mathrm{C}$ are $=$.

Hence, if we take our dividend and divisor for obtaining M 6 , from terms of the $p 3$, a like process applied to the $p 4$ will give M 8. But such process in obtaining a dividend and divisor from terms of a major $p$ of more sides than the $p 4$, will not be likely to give $\mathrm{M}$ of a $p$ of twice the sides. 'Nevertheless, any 
process applied to a $p$ other than the $p 4$, that will give $M$ of a $p$ of twice the sides, will, when applied to the $p 4$, give M 8 .

In the series commencing with the $p 3, \mathrm{M} 6$ is $\mathrm{M}$ of the first secondary $p$ obtained in the series; and in the series commencing with the $p 4, M 8$ is $M$ of the first secondary $p$ obtained in the series; and M 6 and M 8 may both be obtained by means of the popular dividends and divisors; and in either case, $M$ of the secondary $p$ is below a mean between $\mathrm{M}$ and $\mathrm{C}$ of the $p$ of half the sides; and the difference between a mean between $M$ and $\mathrm{C}$ of the $p 3$ and $\mathrm{M} \mathbf{6}$, is greater than that between a mean between $\mathrm{M}$ and $\mathrm{C}$ of the $p 4$ and $\mathrm{M} 8$.

But, by any system, the difference between $M$ and C of a $p$, and $M$ of a $p$ of twice the sides, eventually becomes extinguished; for by the popular method, it must be extinguished in the circle; and few things can be made more manifest than that such difference is extinguished in 2 . Neither will it be contended that $\mathrm{M}$ is eventually above a mean between $\mathrm{M}$ and $\mathrm{C}$ of a $p$ of half the sides.

But this remarkable fact should not be disregarded, that while we find $M$ in 2 a mean between $M$ and $C$ of a $p$ of half the sides, we also find, that if a supposed progression be farther continued, (and for want of a proper equation of polygonal capacities, such may be the case,) $M$ will forever after continue to be a mean between $\mathrm{M}$ and $\mathrm{C}$ of a $p$ of half the sides.

Hence, if we assume a mean between $M$ and $C$ to be the mean place of $\mathrm{M}$ of twice the sides, and institute a polygonal progression upon such assumption, we shall see how much determined a mean between $M$ and $C$ is to coincide with the other polygonal quantities in 2 .

By such process then, the true and mean place of $\mathrm{M}$ will be found to coincide in $2,-$ and will remain so ever after; hence, if they have not coincided before arriving at 2 , then after arriving at $\underset{2}{2}$, the law of progression must be changed, in order that the progression may be continued farther.

But I propose to show that $M$ becomes a mean between $M$ and $\mathrm{C}$ of $\mathbf{a} p$ of half the sides, before we arrive at 2 ; and hence, that the above process is only given by way of the reductio ad absurdum.

But as it was manifest, that the difference between $M$, and $M$ and $C$ of a $p$ of half of the sides, must eventually be extinguished, the popular methods have essayed to do it by a succession of dividends, and corresponding divisions; in which case, each successive dividend and divisor must depend upon the correctness or the incorrectness of the preceding processes. 
Hence, if the process be erroneous, the result must be so too, and the final quantities will not be found in their true places.

Now, with a view to some further examination of the popular mode, by means of dividends and divisors, I will simplify it by translating it to a correspondence with some unity of purpose.

Thus, the popular method essays to obtain $M$ of the prime circle, by a constant division of the product of $M$ by $A$, (which is but the square of $\mathrm{C}$,) by half the sum of $\mathrm{A}$ and $\mathrm{C}$, namely, by an equal mean between $\mathrm{A}$ and $\mathrm{C}$,- supposing that such process would serve, from the $p 4$ to the circle, to give $M$ of a $p$ of twice the sides.

But it will be my business to show that the course pursued conducts truly no farther than the rugged octagon, - that $p$ whose perimeter has transcendental capacities.

Precisely the same continued and final results will be obtained as in the above process, if we divide the product of $M$ by $D$, or of $C$ by $B$, by half the sum of $B$ and $D$. Hence, we find that $B$ and $D$ need not be excluded from participating in a polygonal progression, with a view to obtain the quadrature.

So upon the same principle, (if correct,) $m$ of any given $p$, (of whatever dimensions, ) $\div$ by half the sum of $d$ and $b$ of such $p$, would give $\mathrm{M}$ of a $p$ of twice the sides.

But, perhaps, the simplest of these methods is that in which $\mathrm{B}$ and $\mathrm{D}$ are mostly used,- as by dividing the product of $\mathrm{M}$ by $\mathrm{D},-$ or $\mathrm{C}$ by $\mathrm{B}$, by half the sum of $\mathrm{B}$ and $\mathrm{D}$, and by such process we certainly obtain M 6 and M 8 .

Now the product of $\mathrm{M}$ by $\mathrm{C}$ of the $p 4 \div$ by half such $\mathrm{M}$ and $\mathrm{C}$, gives $\mathrm{M} 8,-$ so the product of $\mathrm{M}$ by $\mathrm{C}$ of the $p 3 \div$ by half of such $M$ and $C$, gives $M 6$; but such process cannot avail farther; for $\mathrm{M}$ by $\mathrm{C}$ of the $p 8 \div$ by half such $\mathrm{M}$ and $\mathrm{C}$, gives $.783100,-$ or even less than the popular $\mathrm{M}$ of the prime circle; which intimates very strongly, the extraordinary capacity of $\mathbf{M}$ 8. And yet nothing is more manifest, than that in the final result, the product of $M$ by $C \div$ by half the sum of such $M$ and $\mathrm{C}$, gives $\mathrm{M},-$ or $\mathrm{M}$ of twice the sides, if we so choose to consider it; and if such progression were conducted on the popular method, the final $M$ would be far below that of the popular final $\mathrm{M}$, for the disproportion between the dividend and divisor would be even greater than by the popular method. Hence, from the $p 8$ to the circle, there is required an equation of perimetric $c a$ pacily.

Various, indeed, (however erroneous the process and results,) may be the methods of conducting a progression by means of a constant succession of dividends and divisors. Thus, the pro- 
duct of $\mathrm{M}$ by $\mathrm{D},-$ or of $\mathrm{C}$ by $\mathrm{B} \div$ by half the surn of $\mathrm{C}$ and $M$, will give the same continued and final results, as if the product of $\mathbf{B}$ by $\mathbf{D}$ be $\div$ by half the sum of such $B$ and $D,-$ and neither of these progressions, if thus conducted, will give the termini below 2 ; for when $M$ and $D$ coincide in 2 , (as they must by any method,) the divisor, namely, half the sum of $\mathbf{M}$ and $\mathrm{C}$, cannot be above ${ }_{2}^{2}$, unless we conceive $\mathrm{C}$ to be above $\mathrm{M}$.

Now in any $p$ between the $p 4$ and circle, $\mathrm{A}$ by $\mathrm{B} \div$ by half of $\mathrm{A}$ and $\mathrm{B}$ will give less, than when $\mathrm{M}$ by $\mathrm{D}$ is $\div$ by half of $\mathrm{M}$ and $\mathrm{D}, \mathrm{-}$ for the reason that an equal mean between $\mathrm{A}$ and $\mathrm{B}$, is greater than an equal mean between $\mathrm{M}$ and $\mathrm{D},-$ while a mean proportional between $A$ and $B$ is the same as that between $M$ and $D$. Nevertheless, the only point of coincidence of $A$ and $B$, or of $M$ and $D$, is in 2 , by any system.

Again, the product of $\mathrm{C}$ by $\mathrm{D} \div$ by half of $\mathrm{A}$ and $\mathrm{D},-$ is identical in result, to that when $M$ by $D$ is $\div$ by half of $C$ and $B$.

Now if we denote the difference between C 8 and D 8,- or between $\mathrm{M} 8$ and $\mathrm{B} 8$, by $\frac{1}{\text {, }}$ - - then the product of $\mathrm{C} 8$ by $\mathrm{D}$ 8 is 1 above the square of C $8,-$ and half the sum of A 8 and $\overline{\mathrm{D}} 8$ will consequently be $\frac{1}{2}$ above the popular divisor, namely, half of A 8 and C 8, - and hence, the quotient will be $\frac{1}{2}$ above the popular quotient, - that is, by such process, M 16 would be $\frac{1}{2}$ above the popular M 16, and it is manifest that such process would diminish the difference between $\mathrm{M}$ and $\mathrm{B}$, or between $\mathrm{C}$ and $\mathrm{D}$, while the popular mode serves to increase the difference between $\mathrm{C}$ and $\mathrm{D},-$ or between $\mathrm{M}$ and $\mathrm{B},-$ or to enlarge the quantity denoted by $\stackrel{1}{\rightarrow}$, or cause it to increase from the $p 8$ to the circle.

Whether we $\div$ the product of $\mathrm{C}$ by $\mathrm{D}$ by half of $\mathrm{A}$ and $\mathrm{D},-$ or the product of $M$ by $D$ by half of $C$ and $B$, the divisor is always to a mean between $M$ and $D$, as such mean is to the quotient. Hence, where the divisor becomes the adverse of $D$, the quotient will be in $2,-$ for a mean between $M$ and $D$, is a mean between the divisor and quotient, - and 2 is the farther of two means from $D$ to a mean between $M$ and $D$.

If then a progression must be carried on by means of cunstant dividends and divisors, (which is at least problematical,) there seems no good reason why diametric quantities may not share in the progression, upon any system whatever. And as the divisor is to the square root of the dividend, as such root is to the quotient, hence, if $\mathrm{M}$ and $\mathrm{D}$ of any given $p$ below the $p 4$, being $\div$ by half of such $M$ and $D$, will give $M$ of a $p$ of twice the sides, then our conceptions at a certain stage of the process will be right; namely, when $M$ and $D$ coincide in $\underbrace{2}$, 
in which case an equal and a rational mean between them would be the same; and no further progress could be made; and similar may be shown to be the results of other progressions.

Nevertheless, all agree that the difference between the divisor, and $B$ of the $p$ of twice the sides of that from which the dividend is obtained, may be eventually equated to 0 ; or that they may eventually coincide. But I have sufficiently treated of polygonal progressions, by means of constant or successive dividends and divisors.

\section{SECTION THIRD.}

Inasmuch as the point denoted by 2 assumes so much consequence, in a consideration and comparison of the prime and primal polygons, it may not be improper to note some few phenomena in respect to it.

Thus, when $m$ of any given $p$ is made the adverse of $\mathrm{D}$ of a like $p$, the area of such given $p$ is 2 . Hence, if $m$ of a given $p$ and $\mathrm{D}$ of a like $p$ be adverse, the product of one area by the other is half of 2 ; but if $m$ of a $p 4$ and $b$ of a $p 4$ be adverse, the product of one area by the other is one fourth of 2 ; while if $m$ of one circle and $b$ of another circle be adverse, the product of one area by the other is half of 2 .

If 2 be the farther of two means from one given point to another, the point farthest from ${ }_{2}^{2}$ is half the square of the reciprocal of the nearest point; hence $M$ is always half the square of the reciprocal of $\mathrm{D}, \mathrm{A}$ of $\mathrm{B}$, and $\mathrm{C}$ of $\mathrm{B}$ of twice the sides.

The adverse of $\mathrm{M}$ is always $\overparen{4}$ below $d$, when $m$ is 1 . So the adverse of $\mathrm{C}$ is always $\overparen{4}$ below $b$, when $m$ is 1 . So also, when $\mathrm{D}$ of a $p$ is the adverse of $\mathrm{M}$ of a like $p$, the area of such $p$ is $\overparen{4}$ below $d$. So when $b$ of a $p$ is the adverse of $\mathrm{A}$ of a like $p$, the area of such $p$ is $\overparen{4}$ below $b$.

The area of any given $p$ is half the square of a mean between such area and $2 . ;$ hence $M$ is half the square of a mean between such $\mathrm{M}$ and 2.

If $m$ of one $p$ and $d$ of a like $p$ be $=$, the product of one area by the other is the fourth power of such $m$ or $d$.

Twice the area of any given $p$ is the square of $b$ of an equal square, with that of the given $p$; hence, $b$ of an equal square, with that of any given $\mathrm{M}$, is $=m$ of a like $p$ with that denoted by $\mathrm{M}$; whose area is 2 .

$M$ and $D$ coincide in 2 ; hence, when $D$ is in $m$ is the square of $d$, and the area is the third power or cube of $d$. Hence $m$ of such $p$ is the third root of the square of the area. 
If If two points be adverse, the reciprocal of either is $\overparen{4}$ above the other.

The product of B by $\mathbf{D}$ of any $p$ is half of $b$ of a like $p$ whose $m$ is 1 .

The square root of $\mathrm{C}$ is $\mathrm{I}$ above a mean between $\mathrm{C}$ and

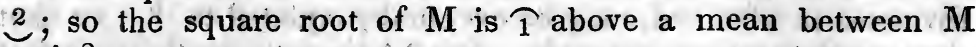
and 2 .

If $b$ of one circle and $m$ of another, $b$ of one $p 4$ and $m$ of another, and $b$ of one $p 3$ and $m$ of another be $=$ in one and the same point, the product of one area of the circle by the other, will be double the product of one area of the $p 4$ by the other, and quadruple the product of one area of the $p 3$ by the other.

The square root of $\mathbf{A}$ is to $\mathrm{C}$ as the square root of $\mathrm{M}$ is to $\mathrm{M}$.

Twice the square of $\mathrm{C} 4$ is the equal reciprocal of $\mathrm{M} 4$; so twice the square of $\mathrm{C} 8$ is the equal reciprocal of $\mathrm{M} 8$. But twice the square of $\mathrm{C}$ of a $p$ of more sides than the $p 8$, is more than the equal reciprocal of $M$.

Hence $\mathrm{M} 4$, plus twice the square of $\mathrm{C} 4$, is 2 ; and $\mathrm{M} 8$, plus twice the square of $\mathrm{C} 8$, is 2. But in any $p$ of more sides than the $p 8, \mathrm{M}$ plus twice the square of $\mathrm{C}$, is more than 2 .

$b$ of a major polygon is the reciprocal of $d$ of a like minor polygon; and when $\mathrm{M}$ is in $2, b$ is $\widehat{2}$ above $\mathrm{B}$ of a like $p$, and $b$ of a like minor $p$ is then 2 above $C$.

The square root of $b$ of any major $p$ is $b$ of a $p$ of twice the sides, whose area is $=$ the area of the given prime $p$.

The square root of $d$ of any minor $p$ is $d$ of a like $p$, whose area is $=\mathrm{C}$ of the given minor $p$.

$\mathrm{D}$ is the reciprocal of $m$ of a like $p$, whose area is 2 .

The product of $m$ by $b$ of a $p 3$, is $\widehat{6}$ above the area; the product of $m$ by $b$ of a $p 4$, is $\widehat{3}$ above the area; and the product of $m$ by $b$ of a circle is equal the area.

If $m$ of the $p 4$ be $=M$ of any given $p$, the square of $b$ of such $p 4$ is the reciprocal of half the square of $b$ of the given $p$ whose $m$ is 1 .

$\mathrm{D}$ of any given $p$ is the reciprocal of $b$ of a $p 4$, whose area is $=\mathrm{M}$ of the given $p$.

$\mathrm{A}$ is to $\mathrm{C}$ as the square root of $\mathrm{A}$ is to the square root of $\mathrm{M}$; hence, $\mathrm{C}$ is to $\mathrm{M}$ of twice the sides as the square root of such $\mathrm{C}$ is to the square root of $M$ of twice the sides.

$\mathrm{M}$ by $\mathrm{C}$ of the $p 4$ is $=m 4$ when the area is .5 ; and when $\mathrm{M}$ and $\mathrm{C}$ of a $p 4$ are adverse, $\mathrm{M}$ by $\mathrm{C}$ is $=m$ of a $p$ of twice the sides, whose area is .5 . 
But $M$ by $C$ of any $p$ between the $p 4$ and the $p$ whose $M$ and $\mathrm{C}$ are adverse, is less than $m$ of a like $p$ whose area is .5 .

Half of the higher of two reciprocal points is the reciprocal of twice the lower point.

A mean between $\mathrm{M}$ and $\mathrm{C}$ of the $p 4$ is half of a mean between $b$ and $d$ of the $p 4$, whose area is 2 ., and the same with the $p$ whose $\mathrm{M}$ and $\mathrm{C}$ are adverse, and each of them is half of $b$ of a $p$ of twice the sides, whose area is 2 ; hence, $\widehat{6}$ above 2 is a mean between $b$ and $d$ of a like $p$ of that whose $M$ and $C$ are adverse, whose area is 2 . Consequently, $\widehat{6}$ above 2 is $b$ of a like $p$ to that whose $\mathrm{M}$ is in 2 , whose area is 2. ; and, consequently, if $\mathrm{M}$ of a $p$ be below, ${ }^{2}$, a mean between $\mathrm{M}$ and $\mathrm{C}$ will be less than half of a mean between $b$ and $d$ of a like $p$, whose area is 2.

If the area of any given $p$ is the third power, or cube, of any given point, such area is a mean between $d$ of such $p$ and $m$ of a like $p$, whose area is $=$ such point.

So the third power of $\mathrm{D}$ of any given $p$ is to .5 , as such $\mathrm{D}$ is to $\mathrm{M}$ of a like $p$.

So the third power of $\mathrm{B}$ of any given $p$ is to .5 , as such $\mathrm{B}$ is to $\mathrm{A}$ of a like $p$.

Hence, the third power of $d$ of a $p$, of whatever dimensions, is to the area of such $p$ as such $d$ is to M of a like $p$.

So the third power of $b$ of a $p$, of whatever dimensions, is to the area of such $p$ as such $b$ is to A of a like $p$.

So also, the third power of $m$ of any given $p$ is to the area of such $p$ as such $m$ is to the reciprocal of $M$ of a like $p$.

The product of the area of any given $p$, of whatever dimensions, by $\mathrm{M}$ of the prime circle, is = the product of the area of the circumscribing circle of the given $p$, by $\mathrm{A}$ of a like $p$ to the given $p$.

When $d$ of any given $p$ is $\underset{2}{2}$, the cube of the area of such $p$ is one fourth of the cube, or third power, of $\mathrm{M}$ of a like $p$.

So the third or cube root of the square of $\mathrm{M}$ of any given $p$ is $m$ of a like $p$, when the area is the third root of such M.

The area of any given $p$ is to the square of $d$ of $\operatorname{such} p$, as M of a like $p$ is to 1 .

So the area of any given $p$ is to the square of $m$ of such $p$ as $d$ when $m$ is 1 ., is to 1 .

When the area of a $p$ is 1 , the product of $d$ by $b$ is $=b$ of a like $p$, whose $m$ is 1 .

Such, then, are a few of the facts, corollaries, and consequents, flowing from that vast store-house of demonstrated principles, which the world has accumulated.

And would the prescribed limits of the work permit; much 
curious evidence might be deduced, in favor of my determination of the quadrature, from a consideration of the sides of polygons, (which I denote by V,) in which the sum of the squares of all the several sides of a regular polygon, is a mean between the square of one side and the square of the entire perimeter. Thus, when $\mathrm{V}$ of the $p 4$ is 1 ., the area is 1 ., or $=d 4$ when $m 4$ is 1. When V 8 is 1 ., the area is = four times $d 8$ when $m 8$ is 1.; and when $\mathrm{V} 16$ is 1 , the area is = sixteen times $d 16$ when $m 16$ is 1 ., \&c.

In such investigation, as also in a consideration of the sum of all the powers of certain polygonal quantities, we should discover the inclination of the measures of the $p 8$ to be governed by equal means, in lieu of proportional means. Thus, the sum of all the powers of the square of $\mathrm{C} 8$ is $=d 8$ when $m 8$ is 1 ; the' sum of all the powers of A 8 is twice $d 8$ when $m 8$ is 1 .; so the sum of all the powers of M 8 is four times $d 8$ when $m 8$ is 1., or four times the reciprocal of M 8, \&c. Nevertheless, the rugged octagon, when unfolded, presents great mathematical beauties; and number 8 , amongst numerical quantities, is certainly a conspicuous number, in its constant application in the development of physical laws by the aid of numbers. I will here suggest, (before proceeding further with explanatory matter,) a simple polygonal progression, based upon the principle, that any given point, either above or below ${ }^{2}$, is half the square of $\overparen{4}$, above a mean between such point and 2 ; and hence, the progression will be alike applicable to all polygons, from the $p 3$ to the circle, and is designed to approximate $D$ and $B$ to a coincidence.

$\mathrm{B}$ of any given $\boldsymbol{p}$ is half the square of $\widehat{4}$ above $b$ of a like $p$, whose area is $\widehat{4}$ below the adverse of $\mathrm{B}$ of the given $p$; and $\mathrm{D}$ of any given $p$ is half the square of $\overparen{4}$ above $d$ of a like $p$, whose area is $\overparen{4}$ below the adverse of $\mathrm{D}$ of the given $p$.

1 is a mean between B 4 and 2 , and B 4 is half the square of 4 above 1 ; and when $b 4$ is 1 , the area of such $p 4$ is $\overparen{4}$ below the adverse of $B 4$.

So D 4 is $3^{3}$, and is half the square of $\widehat{4}$ above a mean between D 4 and 2 ; and when $d 4$ is a mean between D 4 and 2 , the area of such $p 4$ is $\widehat{4}$ below $\underset{\mathcal{G}}{1}$, or is $\widehat{4}$ below the adverse of D 4.

Hence, such progression, by an infinite series, will extinguish the mean between $B$ and ${ }^{2}$, upon the one hand, and between $D$ and 2 upon the other; nor can the progression proceed further. And it is manifest that, at the end of the progression, either in respect to $B$ or $D$, that the area will be .5 , or $\widehat{4}$ below $B$ or $D$, namely, $\overparen{4}$ below 2 . 
From the fact, that the product of the perimeter of any regular $p$, by one fourth of the diameter of the inscribed circle of such polygon, gives the area of such $p$, it follows as a corollary, that if the square root of such perimeter be taken as the hypothenuse of a right angled triangle of two equal legs, or sides, that the square of the distance from the centre of the base to the right angle, (namely, the square of the altitude, ) will be $=$ the area of the given $p$. Let such perimeter, then, be the perimeter, of a major polygon; in which case, the square of the altitude will always be $=\mathrm{M}$ of the given $p$.

In such case, the base will always be $=m$ of a like $p$, whose area is 4 ; one of the equal sides will be $=m$ of a like $p$, whose area is 2 ; and the altitude will be $=m$ of a like $p$, whose area is 1. ; and the square of the altitude will be $=\mathbf{M}$ of a like $p$; one of the equal sides will be $=b$ of a $p 4$, of equal area with that of the given $p$, or will be a mean between 2 . and $\mathrm{M}$ of the given $p$; and when $\mathrm{M}$ of the given $p$ is $\underbrace{2}, b$ of an equal $p 4$ will then be the reciprocal of 2 .

1 is used to denote the difference between $M 8$ and $B 8$, or between $\mathrm{C} 8$ and $\mathrm{D}$; which difference is extinguished, or made $\underline{0}$ in the circle. If, then, 1 be a diminishing quantity from the $p 8$ to the circle, it ought to be so denoted in the course of the progression; thus, if in the $p 16$ it shall be but a proportional half, for instance, of what it is in the $p 8$, and so on, the same should appear, as thus, $1, \frac{1}{2}, \frac{1}{4}$, \&c.; for if the diminishing quantity be constantly denoted by 1 , we shall be inclined to conceive the same difference to exist between $\mathbf{M}$ and $\mathbf{B}$ or $\mathbf{C}$ and $\mathrm{D}$; when the same is wholly extinguished, as existed in the $p 8$.

And, in fact, when $\mathbf{M}$ and $\mathbf{C}$ become adverse, although they then are in actual coincidence, we cannot well get rid of the conception, that $\mathrm{M}$ is $\underline{2}$ above $\underbrace{2}_{2}$, and $\mathrm{C} \underline{2}$ below ${ }_{2}^{2}$; for as we still retain in our conceptions the order of the progression, we still conceive the terms as being separate, and their distance as being the same as previously.

\section{SECTION FOURTH.}

It will not, probably, be doubted or denied, that the order in which the four prime denotations progress, from the $p 4$ to the prime circle, or to the coincidence of $\mathrm{C}$ and $\mathrm{M}$ and the coincidence of $\mathrm{D}$ and $\mathrm{B}$, is as follows, namely, $\mathrm{C}: \mathrm{D}:: \mathrm{M}: \mathrm{B}$, or $\mathrm{C}: \mathrm{M}:: \mathrm{D}: \mathrm{B}$; for such is the popular progression and final determination: The difference between $M$ and $B$ and between $\mathrm{C}$ and $\mathrm{D}$ being 0 in the $p 4$, but greater in the $p 8$ than in the $p 6$. 
Neither will it be denied that, by the popular method, the actual difference between $\mathrm{M}$ and $\mathrm{B}$ and between $\mathrm{C}$ and $\mathrm{D}$, is increased from the $p 4$ to the circle; so that the difference is even greater (saying nothing about ratio,) in the circle than it is in the $p 8$.

Neither will it be denied, that the progressive ratios of $M$ and $D$ may coincide in ${ }^{2}$, and that they cannot, upon any system or principle, coincide in any other point or position; and that the same will be the case in respect to $A$ and $B$, or $C$, and $B$ of twice the sides, in case they are permitted to progress thus far; for such is manifestly the law of progression, by any system or method.

So it is manifest, also, that by the general law of progression, when the progressive ratios of $M$ and $D$ coincide in 2 , if $C$ and $B$ do not then coincide with $M$ and $D$, they will be adverse; so also, if $M$ and $C$ of a $p$ of half the sides of that whose $M$ and $D$ are in $\underset{2}{2}$, are not also in $\underset{\checkmark}{2}$, they must necessarily be adverse.

Hence, when $M$ and $D$ coincide in 2 , if a like or a similar difference still prevails between $M$ and $B$ and between $C$ and $D$ as in the $p 8$, the formula for the four prime denotations, for the $p$ whose $M$ and $\mathrm{D}$ are in 2 and the $p$ of half the sides, will be thus : $C: D C: D M: B: M: B$. But if, when $M$ and $D$ coincide in ${ }^{2}$, the difference between $M$ and $B$ and between $C$ and $\mathbf{D}$ is $\mathbf{0}$, or is equated to $\underline{0}$, then all the above eight denotations are in 2 ; or, if we choose so to consider it, those denotations belonging to the $p$ whose $\mathrm{M}$ and $\mathrm{D}$ apparently coincide in 2 , are extinguished, by the coincidence of those of half the sides, which may be shown to be universally the case in respect to all denotations (of whatever kind) which apparently coincide in 2 ; and hence, we may either consider them as vanishing, or becoming extinguished by like denotations of half the sides, in the point of apparent coincidence; or we may consider them as still existing in coincidence with those of half the sides.

And to show that such phenomena must take place, (confining our investigations wholly to major, minor and primal polygons,) one side of the minor $p 4$, when properly equated and adjusted, is as well the base of two adjoining sides of the major $p 8$, as of two adjoining sides of the minor $p 8$; and the like is true, in respect to the $p 8$ and $p 16$, and so on, ad infinitum, until one side of a minor $p$ is = two sides of either a major or minor $p$ of twice the sides; or, rather, until all numeral difference between them is extinguished.

The application, thus far, in cases in which one and two sides have been considered, has been general, and is adapted to any 
series of polygons; but, in order to avail ourselves of the full benefits of the phenomena, we must confine ourselves to that series of polygons which commences with the $p 4$; for the reason, that in such a series $\mathrm{M}$ or $\mathrm{C}$ of any $p$, (except that of the $p 4$, ) is composed of an even number of sides ; and hence, from the $p 8$ onward to the circle, the phenomena apply equally as well to $\mathrm{M}$ or to $\mathrm{C}$, as to one or two sides, which is not the case with any other series than that commencing with the $p 4$. Hence, the commencement of our process is properly with the $p 8$; and especially, when we wish to show the progress of a polygonal progression downward, towards $M$ of the prime circle.

Thus $\mathrm{C}$ of the $p 8$ may be said to span both $\mathrm{C}$ and also $\mathrm{M}$ of the $p 16$, and so on, until C becomes $=\mathrm{C}$, as also $\mathrm{M}$, of a $p$ of twice the sides, which can only occur in the circle; and the consequence necessarily is, that in either $p, \mathbf{C}$ is $=\mathbf{M}$, which may be called the fourfold chord of the periphery.

Hence $M$, in descending, must necessarily become $=M$, as also $=\mathrm{C}$ of a $p$ of twice the sides; and the only question is, in what numerical point or position shall this fourfold chord be formed or situated? If it be in $\underset{2}{2}$, then B and D final are in 2 ; if it be above 2 , then $\mathrm{D}$ and $\mathrm{B}$ final will be below 2 and if it be below 2 , then $D$ and $B$ final will be above 2 ; for if it be not in ${ }_{2}^{2}$, then ${ }_{2}^{2}$ will be the farther of two means from the coincidence of $\mathrm{M}$ and $\mathrm{C}$ to that of $\mathrm{B}$ and $\mathrm{D}$.

If $M$ and $C$ coincide in 2 , the difference between $M$ and $B$ and between $C$ and $D$ will have become equated to $\underline{0}$. If $M$ and $\mathbf{C}$ coincide below $\underbrace{2}_{2}$, such difference will remain, or has been increased or diminished from what it was in the $p 8$, accordingly as it shall be in the circle. And if $\mathrm{M}$ and $\mathrm{C}$ shall coincide above $\underset{2}{2}$, then such difference will have been extinguished, and will have again increased in the opposite direction. And certainly I will not contend in favor of the last position, but it is my business to contend against the second.

This fact, alone, then, would seem to present an insurmountable difficulty in the way of the popular mode, namely, when the progressive ratios of $M$ and $D$ coincide in 2 ; if we attempt to extend the progression farther, $M$ and $B$ will in future progress by the same ratio, and $\mathrm{C}$ and $\mathrm{D}$ will also progress by the same ratio, which is contrary to the popular law of progression, in which the difference between $M$ and $B$, as also between $C$ and $\mathrm{D}$ is constantly increasing from the $p 8$ to the circle. And certainly the very law of progression itself, ought not to be required to give way, under any circumstances. Besides, $M$ and $\mathbf{B}$ are properly regarded as descending quantities; and two 
separate quantities, descending by the same ratio, must necessarily converge.

Hence, it is impossible that the progression can be extended after $\mathrm{M}$ and $\mathrm{D}$ coincide, without an alteration of the law of progression. If, then, the law of progression is not altered in $\underbrace{2}_{2}$, then $M$ and $C$, and $B$ and $D$ coincide in 2 .

We perceive, that $\mathrm{C}$ and $\mathrm{C}$ and $\mathrm{M}$ of a $p$ of twice the sides, (in the series commencing with the $p 4$,) preserve their relative situations from the $p 8$ and $p 16$ to the circle; and D of the $p$ whose $\mathrm{M}$ and $\mathrm{C}$ are adverse, and $\mathrm{D}$ and $\mathrm{B}$ of the $p$ of twice the sides, (namely, the $p$ whose $\mathrm{M}$ and $\mathrm{D}$ are in ${ }^{2}$,) have the same relative situations, in respect to each other, as $\mathrm{C}$ of a $p$ whose $\mathrm{M}$ and $\mathrm{C}$ are adverse, and $\mathrm{C}$ and $\mathrm{M}$ of the $p$ of twice the sides, have.

I have said, that from the $p 8$ to the circle the same relative situation, between $\mathbf{C}$, and $\mathbf{C}$ and $\mathbf{M}$ of a $p$ of twice the sides, is constantly preserved; and it may be of consequence to know, whether from the $p 8$ to the circle the same relative situation is constantly preserved between $\mathrm{C}$ and $\mathrm{M}$ of a $p$ and $\mathrm{C}$ and $\mathrm{M}$ of a $p$ of twice the sides.

A mean between $C$ and $M$ is to a mean between $D$ and $B$, as $C$ is to $D$ or as $M$ is to $B$; or a mean between $M 8$ and $C 8$ is 1 below a mean between B 8 and D 8 ; hence, if M 16 be a mean between $M 8$ and $C 8$, it is 1 below a mean between $B$ and $\mathrm{D} 8$.

Now M 8 is a mean between M 4 and 2 below D 4, and M 8 is also a mean between $\mathrm{C} 4$ and 2 below B 4 ; and if $\mathrm{M} 16$ be a mean between M 8 and $\mathrm{C} 8$, then the quantity denoted by 2 must be constantly halved. Now. M 16 is to a mean between $\mathrm{M} 8$ and $\mathrm{C} 8$ as it is to a mean between $\mathrm{C} 8$ and M 8 , for they are one point, and so on. So M 16 is to a mean between M 8 and 1 , below $\mathrm{D} 8$, as it is to a mean between $\mathrm{C} 8$ and 1 below B 8, for they are one and the same point.

If, then, M 16 is a mean between $M 8$ and 1 below D 8 or C 8 , and 1 below B 8, we then have the terms of a progression from the $p 4$ to the circle, in which, from the $p 8$ to the circle, the same relative position will constantly hold between $\mathbf{M}$ and $\mathbf{C}$ of any $p$ and $M$ and $C$ of a $p$ of twice the sides; and it would seem, in reality, as if such progression was corroborated by the popular determination.

If, then, the $p 4$ be called the primitive $p$ of the series, and all the other polygons of the series be called secondary polygons, then, while $\mathrm{C} 16$ is a mean between $M 16$ and $C 8, M 16$ will be a mean between M 8 and C 8, and so on to the circle.

Bat to proceed with the progression on the foregoing; $M 8$ is 
a mean between M 4 and 2 below D 4 , and between $\mathrm{C} 4$ and 2 below B 4, (for they are one and the same.) M 16 is a mean between M 8, and 1. below D 8, and between C 8 and 1 below B 8 . $M 32$ is a mean between $M 16$, and $\frac{1}{2}$ below $D 16$, and between C 16 , and $\frac{1}{2}$ below B 16 , and so on.

Such being the case it is manifest that all the four denotations must coincide together, which must of necessity be in 2 . Or, on the same principle, we have this progression: A 8 is to $M 8$ as $\mathrm{M} 8$ is to $\frac{2}{2}$ below $\mathrm{B} 4$; A 16 is to $\mathrm{M} 16$, as $\mathrm{M} 16$ is to 1 below $B 8$, and so on. Or thus; M 4 is to $M 8$, as $M 8$ is to $\frac{2}{2}$ below $\mathrm{D} 4, \mathrm{M} 8$ is to $\mathrm{M} 16$, as $\mathrm{M} 16$ is to 1 below $\mathrm{D} 8$, and so on.

Thus $\mathrm{A}$ and $\mathrm{M}$ would coincide with $\overline{\mathrm{B}}$ of half the sides, and $M$ and $\mathbf{D}$ would coincide with $\mathrm{M}$ of twice the sides. And this entirely comports with the law of progression. So again, $\mathrm{C} 4$ is to $\mathrm{M} 8$, as $\mathrm{M} 8$ is to 2 below $\mathrm{B} 4 ; \mathrm{C} 8$ is to $\mathrm{M} 16$, as $\mathrm{M} 16$ is to 1 below $\mathrm{B} 8$, and so on.

But the popular methods instead of equating $\frac{2}{2}$ to $\underline{0}$, have

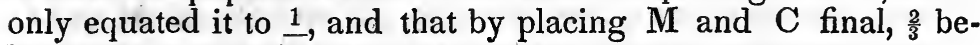
low their proper place.

But a great number of progressions of the kind, and on similar principles, may be instituted - all of which will find their termini in 2 : for $m$ of any given $p$ when the area is .5 , namely, $\mathrm{M}$ by $\mathrm{D}$, or $\mathrm{C}$ by $\mathrm{B}$, of any given $p \div$ by 2 , gives $m$ of a like $p$, when the area is 2 .

$\mathrm{M}$ of any given $p$, is to $b$ when the area is 1 , as $\mathbf{C}$ is to $d$ when the area is 1 , and the proportional difference between $M$ 4 , and $b 4$, when the area is 1 , is less in the $p 4$, than in any $p$ between the $p 4$ and circle; but this proportional difference is the same in the $p 4$ and the circle. Hence the linear capacities are the same in the $p 4$ as in the circle. The difference between $M$ and $b$, when the area is 1 , or between $\mathrm{C}$ and $d$, when the area is 1 , is greater in the $p 8$ than in any $p$ of the series commencing with the $p 4$, and is denoted by 1 ; hence the $p 8$ is the proper $p$ from which to commence our equation of polygonal capacities, whether it be mathematically or geometrically performed.

$\mathrm{M} 8$ is 1 below a mean between M 4 and D 4 , or C 4 and B 4 , and $M 16$ is $\frac{1}{2}$ below a mean between $M 8$ and D 8 , or $C 8$ and $B$ 8 , and so on. And by such progression, it is manifest that all the terms coincide in ${ }_{2}^{2}$.

Now no one will deny but that $\mathrm{M} 8$ is 1 below the square root of $m 4$, when the area of such $p 4$ is .5 ; nevertheless, the popular method has placed $M$ of the prime circle, $\frac{1}{2}$ below the square root of $m$ of the circle whose area is .5 , from which it is manifest that by such method, no progress has been made from the $p 16$ to the circle, but that there has even been a retrograde 
from the truth, occasioned by an increase of the constant divisor in an incorrect proportion to that of the constant dividend.

I have said it might be important to know awhether from the $p 8$ to the circle, $\mathrm{M}$ and $\mathrm{C}$, and $\mathrm{M}$ and $\mathrm{C}$. of a $p$ of twice the sides, constantly retained the same relative position in respect to each other. It is quite certain that they do not from the $p 4$ to the circle. Then draw the circle whose diameter is assumed at unity, and from any point in the periphery, draw one side of the perimeter of the major $p 8$, (namely, one side of $M 8$, ) as a tangent, and such tangent may be said to be the tangent to one side of $\mathrm{C} 8$, and also to turo sides of $\mathrm{M} 16$, as also two sides of C 16, one side of M 16 being merged in such tangent, which side of M 16 so merged, is the tangent of two sides of M 32, of two sides of C 32, and of one side of C 16, - and so on, ad infinitum, until the tangent is extinguished, namely, until $\mathbf{M}$ and $\mathbf{C}$ coincide with $\mathbf{M}$ and $\mathbf{C}$ of twice the sides, or until $\mathbf{M}$ and $\mathbf{C}$ have extinguished $\mathbf{M}$ and $\mathrm{C}$ of twice the sides, thus determining the fact, that from the $p 8$ to the circle, $\mathrm{M}$ and $\mathrm{C}$ and $\mathrm{M}$ and $\mathrm{C}$ of twice the sides constantly retain the same relative situation to each other. And no fact can be made more manifest, than that when $\mathrm{M}$ is in $2, \mathrm{M}$ and $\mathrm{C}$ of half the sides are adverse, and $\mathrm{D}$ of the $p$ whose $\mathrm{M}$ is in 2 is $=\mathrm{M} ; \mathrm{C}$ of such $p$ is a mean between $\mathrm{M}$ of such $p$, and $\mathrm{C}$ of half the sides. $\mathrm{B}$ of such $p$ is a mean between $\mathrm{M}$ or $\mathrm{D}$ of $\operatorname{such} p$, and $\mathrm{M}$ of half the sides.

What then must be the relative situation of $\mathrm{M} 16$ in respect to $\mathrm{M} 8$ and $\mathrm{C} 8$ ? Let legitimate geometry answer the question.

Hence, M 16 is a mean between C 8 and 1 below B 8, or is a mean between $M 8$ and 1 below $D 8$. Or $M 16$ is $\frac{1}{2}$ below a mean between $M 8$ and D 8 - or $\frac{1}{2}$ below a mean between $C$ 8 and B 8. So M 32 is $\frac{1}{4}$ below a mean between M 16 and D 16 , or C 16 and B 16, and so on, ad infinitum, until M finally coincides with $M$ and $D$ of half the sides and with $C$ and $B$ of half the sides. And as before shown, such coincidence is in 2 .

We hence obtain or avail ourselves of the true equation of polygonal measures; but to attempt this without either unity of purpose, or legitimate geometry, would be a hopeless task.

Hence if any final denotation is in $\underset{6}{2}$, they all become final in 2 ; in which case also, if any denotation appears to be in 2 it is there extinguished by a like denotation of half the sides. And it might be a pleasant exercise in mathematics, to multiply auxiliary denotations of other polygonal quantities; as for instance, a mean between $C$ and $D$, which is also a mean between $\mathrm{A}$ and $\mathrm{B}$ - and a mean between $\mathrm{M}$ and $\mathrm{D}$, which is also a mean between $\mathrm{C}$ and $\mathrm{B}$, and is the square root of $m$ of a like $p$ whose area is 5 , or half the square of the reciprocal of $A$, or of $C$, or of $\mathrm{M}$ and $\mathrm{C}$; all of which, by the laws of polygonal progression, 
are seen to progress, or flow into, and to coincide in 2 But I forbear to enter upon it further. It may, however, be suggested, that if we signify the $p$ whose $\mathrm{M}$ and $\mathrm{D}$ appear to coincide in 2 , by placing figure 2 at the right of its denotations, and figure 1 at the right of the denotations of the $p$ whose $\mathrm{M}$ and $\mathrm{C}$ appear to be adverse, then when M 2 and D 2 appear to coincide in $2, \mathrm{C} 1$ and $M 1$ actually coincide in $\underset{2}{2}$, in which case $M 1$ will appear to assume the identical place of $\mathrm{C} 1$, when $\mathrm{M} 1$ and $\mathrm{C} 1$ are conceived to be adverse; and if the progression be conceived to be still continued (for we may conceive a continuance of the progression until we can conceive quantity to be extinguished by constantly halving it) $M$ and $B$ will thence be conceived to ascend, and $\mathrm{C}$ and $\mathrm{D}$ to descend, contrary to the order or law of polygonal progression, which process, or imaginary process, (for it is but imaginary,) will present many curious coincidences and adverse reciprocities by way of the reductio ad absurdum, which I will not here enter upon.

But I will cease to multiply evidence of this description, assuring the world that the store house is yet full, and by no means exhausted by the pittance which I have drawn from it.

$\mathrm{Z}$ may denote a mean between $\mathrm{M}$ and $\mathrm{D}$ or $\mathrm{C}$ and $\mathrm{B}$ of any. given $p$, which is at the same time the square root of $m$ of a like $p$ whose area is .5. Then, from the $p 4$ to the circle, $\mathrm{Z}$ and $\mathrm{M}$ of a $p$ of twice the sides, approximate each other, by any system. Then, $\mathrm{Z} 4$ is $\frac{1}{\text { above }} \mathrm{M} 8, \mathrm{Z} 8$ is $\frac{1}{2}$ above M 16, Z 16 is $\frac{1}{4}$ above $\mathrm{M}$ 32 , and so on, till $\mathrm{Z}$ is $=\mathrm{M}$ of twice the sides.

$\mathbf{Z}$ is the farther of three means from $\mathbf{M}$ to 2 . Hence, $\mathbf{Z}$ cannot be $=\mathbf{M}$ of twice the sides below $\underset{2}{2}$, for in that case $M$ would be below $M$ of twice the sides, which is impossible.

If from the $p \mathrm{~S}$ to the circle, a mean between $\mathbf{M}$ and $\mathbf{C}$ is $\mathbf{M}$ of twice the sides, a progression on this principle will be identical with that by which we say that $\mathrm{Z} 4$ is $\frac{1}{2}$ above $\mathrm{M} 8, \mathrm{Z} 8$ is $\frac{1}{2}$ above $\mathrm{M} 16$, \&c. And then, from the $p 8$ onward, $\mathrm{D}$ is, to a mean between $\mathrm{D}$ and $\mathrm{C}$, as a mean between $\mathrm{M}$ and $\mathrm{D}$ is to $\mathrm{M}$ of twice the sides, or as $\mathrm{Z}$ is to $\mathrm{M}$ of twice the sides, or as $\mathrm{Z}$ is to a mean between $\mathrm{M}$ and $\mathrm{C}$ of the same $p$, which last also applies to the $p 4$.

Again, when $\mathrm{Z}$ becomes a mean between $\mathrm{M}$ and $\mathrm{C}(=\mathrm{M}$ of twice the sides) such mean between $M$ and $C$ (or $M$ of twice the sides) is a mean between $\mathrm{M}$ and $\mathrm{D}$, or $\mathrm{C}$ and $\mathrm{B}$, or $\mathrm{B}$ and $\mathrm{D}$. Then $\mathrm{M}$ is $=\mathrm{B}$, and $\mathrm{C}$ is $=\mathrm{D}$; and $\mathrm{M}$ of twice the sides is also $=\mathrm{B}$ of such $p$ of twice the sides : thus in two intermediate polygons, $\mathrm{M}$ is $=\mathrm{B}$, and when $\mathrm{M}$ is $=\mathrm{B}, \mathrm{C}$ is $=\mathrm{D}$.

Because M 8 is a mean between M4 and 2 below D 4 , it is a mean between $\mathrm{C} 4$ and 2 below $B$. 4 . Because $M 16$ is a 
mean between $\mathrm{M} 8$ and 1 below $\mathrm{D} 8$, it is a mean between $\mathrm{C} 8$ and 1 below B 8 , and so on.

So when $M$ becomes a mean between $M$ and $D$, or $C$ and $B$ of half the sides, it is also a mean between $M$ and $C$ of half the sides. When $\mathbf{M}$ is given, we conceive $\mathbf{M}$ of twice the sides, even though there be no difference between them.

Hence, when $M$ and $D$ coincide, we conceive $M$ of twice the sides, even though it be $=\operatorname{such} M$ in $\underbrace{2}$ as a mean between $M$ and $\mathrm{D}$ in 2 .

In order that $M$ may be a mean between $M$ and $D$ of half the sides, it must be the farther of three means from $M$ of half the sides, to 2 .

$\mathrm{M}$ below 2 , cannot be a mean'between $\mathrm{M}$ and $\mathrm{D}$ of half the sides, for in that case, $M$ of half the sides would be the lower $M$, for $M$ cannot be above $M$ of half the sides.

By no system is $M$ ever above a mean between $M$ and $D$ of half the sides, that is, a mean between $M$ and $C$ is never above a mean between $M$ and $D$.

Whenever $M$ is a mean between $M$ and $D$ of half the sides, it is also a mean between $M$ and $C$ of half the sides, in which case $\mathrm{C}$ and $\mathrm{D}$ are necessarily $=$. So when there is no difference between $\mathrm{M}$ and $\mathrm{B}$, or $\mathrm{C}$ and $\mathrm{D}, \mathrm{a}$ mean between $\mathrm{M}$ and $\mathrm{C}$ of such $p$ is $M$ of twice the sides.

By the popular method, $M$ does not become a mean between $M$ and $C$ of half the sides, except in the circle, and hence, $M$ is always, (from M 16 to the circle,) $\frac{1}{2}$ below a mean between M and $\mathrm{D}$, or C and B. But the fact is too manifest to be doubted, that $\mathrm{M}$ in 2 . is a mean between $\mathrm{M}$ and $\mathrm{C}$ of half the sides.

When $M$ is in ${ }^{2}, Z$ is necessarily in ${ }_{2}^{2}$, being then the farther of three means from $M$ to 2 .

$\mathrm{Z}$ is above $\mathrm{M}$ of twice the sides, until they become $=$. In $2, \mathrm{M}$ is $=\mathrm{Z}$ of the same $p$. Below $\underset{2}{2}, \mathrm{M}$ cannot be $=\mathrm{Z}$ of any $p$. Hence $\mathrm{M}$ cannot be $=\mathrm{Z}$ of half the sides, except when $Z$ and $M$ of the same $p$ are $=$ and such must be the case in the point in which $M$ coincides with $M$ of twice the sides. Such point is in 2 .

The progression may also be carried on thus: The square root of $m 4$, when the area is .5 , is 1 above M 8 . The square root of $m 8$, when the area is .5 , is $\frac{1}{2}$ above $M 16$, \&c., until the square root of $m$, when the area is .5, is $=M$ of twice the sides.

Now it is manifest that the square root of $m$, when the area is .5 , cannot be $=\mathrm{M}$ of twice the sides, in any point above $\underbrace{2}$, for it is only in 2 that it becomes $=\mathrm{M}$ of a like $p$, for a mean between $\mathrm{M}$ and $\mathrm{D}$, (which is the square root of $m$ when the area is .5, ) is above ${ }^{2}$, until $\mathrm{M}$ and $\mathrm{D}$ coincide in ${ }^{2}$. 
It is equally manifest, that the square root of $m$ when the area is .5 , cannot be $=\mathrm{M}$ of twice the sides below ${ }^{2}$, for the reason that the square root of $m$, when the area is .5 , is always the farther of three means from $M$ to 2 ; hence, for the square root of $m$, when the area is .5 , to be $=\mathrm{M}$ of twice the sides, in any point below ${ }^{2}$, would require $\mathbf{M}$ to be below $\mathbf{M}$ of twice the sides, which would be a phenomenon that no one would expect.

Hence it is determined in the most conclusive manner, that 2 is the point in which we must conceive the square root of $m$, when the area is .5 , to become $=\mathrm{M}$ of twice the sides; while in the same point, it is $=\mathrm{M}$ of the same $p$.

Hence, 2 is the very point in which $M$ apparently becomes $=\mathrm{M}$ of twice the sides, which is a phenomenon that apparently must occur in the formation of the periphery of the circle. Nevertheless, such $\mathrm{M}$ of twice the sides does not actually exist, it being but the conception of $M$ of twice the sides of that $M$ which has no sides, or the number of which (if number it may be called) is infinite. And should any require corroborative evidence in favor of my determination that $M$ of the prime circle is in $\underbrace{2}$, let them apply to legitimate geometry.

The equation of the capacities of polygonal measures, properly commences with the $p 8$, in which $p$ there is the greatest difference between $\mathrm{M}$ and $\mathrm{B}$, or $\mathrm{C}$ and $\mathrm{D}$, and this is plainly dictated by geometry. And in reference to the varying capacities of polygonal measures, the $p 4$, or unit polygon, is the perfect standard, the true place of any one of its measures always being in the mean place. But not so in respect to polygons situated between the $p 4$ and its inscribed circle; in which the linear capacities are variable, the true places varying from the mean, and hence requiring a constant equation, the variance being greater in the $p 8$ than in any other $p$.

Thus the variance of the true place of $m 8$ from the mean place, is proportionally twice as great in respect to $d 8$, as it is in respect to $b 8$, and $d S$ is proportionally as much above its mean place, as $m 8$ is below, while $b 8$ is always in its mean place in respect to area, as well in the $p 8$ as in all polygons from the $p 4$ to the circle; for it must be recollected that these varying capacities have regard or reference, to the admeasurment of area.

Nevertheless, the linear capacities of the circle are the same as those of the $p 4$; and hence, in a progression from the $p 4$ to the circle, those varying capacities must be regarded in order to avoid constant and final error.

This must be done by geometrizing wholly by means of lines, (which is the only legitimate geometry,) without any regard whatever to areas, in the course of our progression; for we may 
be assured, that when our lines are properly adjusted and proportioned, that the areas will be so too. But it is too manifest, that if we attempt to progress by means of areas instead of lines, that we shall wholly fail to equate the varying linear capacities of polygons situated between the $p 4$ and its inscribed circle.

I have said that if one final denotation be in 2 , (however the denotations be multiplied, then all the denotations will necessarily coincide together in 2 ; but if any final denotation be not in 2 , then the final denotations will be scattered, some being above 2, and some below, but none in 2 .

And for the purpose of exhibiting the phenomenon, I will still increase the number of denotations expressed by capital letters.

$\mathrm{E}$ may denote half the square of the reciprocal of $\mathrm{A}$, of any given $p$. $\mathrm{F}$ may denote half the square of the reciprocal of $\mathrm{C}$ of any given $p$; namely, half the square of $b$ of a $p$ whose $m$ is $1,-$ and $G$ may denote half the square of the reciprocal of $\mathrm{M}$; namely, half the square of $d$ of a $p$ whose $m$ is 1 . Now half the square of the reciprocal of $\mathrm{C}$ of any given $p$ is $=$ the area of a like $p$ whose $m$ is $=\mathrm{B}$ of a like $p$; and in the $p 8$ such area, or half the square of the reciprocal of $\mathrm{C} 8$, is an equal mean between M 4 and $\mathrm{C} 4$, and is the proper divisor for obtaining M 8, when the dividend is the product of $\mathrm{M} 4$ by $\mathrm{C} 4$, and by such, or a similar divisor does the popular method essay to carry on a progression ; but it will not succeed beyond obtaining $M$ 8. So also, half the square of the reciprocal of $M$ is = the area of a like $p$ when $m$ is $=\mathrm{D}$ of a like $p$.

$B$ then of any $p$ is a mean between $M$ and half the square of the reciprocal of $\mathrm{C}$ of a like $p$, and $\mathrm{D}$ of any $p$ is a mean between $M$ and half the square of the reciprocal of $M$ of such $p$.

$B$ of any given $p$ is the farther of three means from half the square of the reciprocal of A of a like $p$ to 2 - and D of any given $p$ is the farther of three means from half the square of the reciprocal of $\mathbf{M}$ to 2 .

Hence, a mean between half the square of the reciprocal of $\mathbf{C}$ and 2 , is the adverse of $\mathrm{C}$, and a mean between half the square of the reciprocal of $M$ and 2 , is the adverse of $M$.

But I will not further multiply terms, as any one can multiply them at pleasure, and discover a vast variety of polygonal coincidences and reciprocities, very many of which I have examined, but have not here even hinted at.

Let then any denotation which I have signified by a capital letter be final in 2 .

Let $\mathrm{A}$ then, be considered final in $\underset{2}{2}$; then $\mathrm{B}$ is in ${ }^{2}$, for $\mathbf{A}$ and $\mathbf{B}$ coincide in ${ }_{3}^{2}$; then $\mathbf{C}$ of half the sides is final in $\underset{2}{2}$; so $\mathrm{M}$ is final in the point in which $\mathrm{C}$ is final; $\mathrm{D}$ coincides with 
$\mathrm{M}$ of a like $p$ in ${ }^{2}$; so then $\mathrm{D}$ is final in $\underset{2}{2}-\mathrm{Z}$ is final in $\underbrace{2}$, because it is a mean between $\mathrm{M}$ and $\mathrm{D}$, or $\mathrm{C}$ and $\mathrm{B}$. A being in ${ }^{2}$, hence $\mathrm{E}$ is in $\underbrace{2}$, for $\mathrm{E}$ is half the square of the reciprocal of $\mathrm{A}$; and the like may be said in respect to $\mathrm{C}$ and $\mathrm{F}$, or of $\mathrm{M}$ and $\mathrm{G} ;$ - or thus - if $d a$ denote the point in which $d$ and the area of any given $p$ are $=$, which is always the reciprocal of $M$, or is $d$ when $m$ is 1 ; -if $b a$ denote the point in which $b$ and the area of any given $p$ are $=$, which is the reciprocal of $\mathbf{A}$ of a like $p$, then $\mathrm{D}$ is the farther of three means from half the square of $d a$ to $\underset{2}{2}$; hence when D is in $\underset{2}{2}$, half the square of $d a$ is in 2 ; when $\mathrm{A}$ and $\mathrm{B}$ are in $\underset{2}{2}$, half the square of $b a$ is in $\underset{2}{2}$; and when $\mathrm{C}$ is in ${ }^{2}$, half the square of $b a$ of twice the sides is in 2 . Hence, if any denotation be final in $\underset{2}{2}$, there is then a general coincidence of all the special denotations in 2 excepting $d a$ and $b a$, which are then reciprocals of 2 , and is the diameter of an orbit in which the period is .5. I might have here added another very important denotation, signifying a mean between $C$ and $D$, (as $\mathrm{Z}$ does between $\mathrm{M}$ and $\mathrm{D}$,) which denotation may be expressed by $\mathrm{Y}$, which is always the farther of three means from $\mathrm{A}$ to ${ }^{2}$, and the square of which is a mean between .5 and $\mathrm{A}$, of a like $p$; hence, when $\mathrm{A}$ is in $\underset{2}{2}, \mathrm{Y}$ is in $\underset{2}{2}$, at the same time such $\mathrm{Y}$ being a mean between $\mathrm{C}$ and $\mathrm{D}$, which are also in 2 ; so $Z$ in 2 is a mean between $M$ and $D$ when they coincide in ${ }_{2}^{2}$.

Hence 2 seems to be emphatically the goal of the spirit land, and deserves far more consideration than the narrow limits which I have assigned to this work will justify ; it being my design to select a few of the dictums and corollaries from amongst those which I have examined and considered, and those too which are least complex, or may be most easily understood.

But in respect to that focal point denoted by ${ }_{2}^{2}$, at which all polygonal denotations centre and find their home, it is possible that a little farther hovering around it, by way of "closer and closer contact," may not be inappropriate at the close of this chapter.

Now if the difference between $\mathrm{M}$ and $\mathrm{B}$ or $\mathrm{C}$ and $\mathrm{D}$ be con stantly denoted by $\stackrel{1}{\text {, }}$ (as would be the case by the popular method, save that 1 becomes enlarged from the $p 8$ to the circle, by the popular mode,) there will be no difficulty in determining the relative situation of the denotations of any $p$, whenever any two denotations coincide, or those of a $p$ of half the sides, or of twice the sides, \&c. For in such case, conception will always place B $\frac{1}{2}$ above $\mathrm{M}$, and D $\underline{1}$ above $\mathrm{C}$. So $\mathrm{F}$.will be $\underline{2}$ above M, and $\mathrm{G}$ will be 2 above $\mathrm{A}$, by conception; and such is the conception in the finale, or in the circle. Nevertheless, the popular determination furnishes as ample proof as can be desired 
that the progressive ratios of $\mathrm{M}$ and $\mathrm{D}$, of $\mathrm{A}$ and $\mathrm{B}$, of $\mathrm{C}$ and $B$ of twice the sides, of $D$ and $Z$, of $B$ and $Y, \& c . \& c$., have their point of coincidence in 2 .

Let $M$ and $D$ then coincide in 2 , and we conceive $B$ to be $\frac{1}{B}$ above $M$, and $D \underline{1}$ above $C$; hence, when $M$ and $D$ are in $2, \bar{B}$ and $\mathbf{C}$ are adverse. But as $\mathbf{C}$ is $=\mathrm{A}$ of twice the sides, hence, $\mathrm{C}$ of the $p$ whose $\mathrm{M}$ is in $\underset{2}{2}$, is a mean between $\underbrace{2}$, and $\mathrm{C}$ of half the sides; that is, $\mathrm{C}$ of half the sides will be conceived to be $\frac{2}{2}$ below 2 , and as $\mathrm{D}$ is 1 above $\mathrm{C}$, hence, $\mathrm{C}$ of the $p$ whose $M$ is in ${ }^{2}$ is $=\mathrm{D}$ of a $p$ of half the sides, (and here I will make the allegation, that $\mathrm{D}$ can never be above $\mathrm{C}$ of twice the sides, nor can $M$ be below $B$ of twice the sides.)

Thus we find $\mathrm{D}$ to be 1 below $2-$ and as 2 is the farther of two means from $M$ to $\mathrm{D}$, hence $\mathrm{M} 1$ is conceived to be $\underline{2}$ above 2 , or the adverse of $\mathrm{C} 1$.

Hence, $\mathrm{M}$ and $\mathrm{D}$ of the $p 2$ is in $\underset{2}{2}$, and $\mathrm{B}$ and $\mathrm{C}$ of the same $p$ are adverse, and $\mathrm{M}$ and $\mathrm{C}$ of the $p 1$, are adverse; and hence it is easy to assign all the relative denotations of these two polygons.

In such case, as we still conceive the difference between $B$ and $\mathrm{M}$, or $\mathrm{D}$ and $\mathrm{C}$, to be the same as in the $p 8$, (namely, denoted by $\frac{1}{\text { ) }}$ we necessarily conceive $\mathrm{M}$ and $\mathrm{C}$ of the $p 2$ to be separated by the same amount as $\mathrm{M}$ and $\mathrm{B}$, or $\mathrm{C}$ and $\mathrm{D}$.

Hence, if we suppose such difference to be reality, in lieu of mere conception, then the progression must be carried on, ad infinitum; and it is quite manifest that such fancied progression would have a termini nearly (though not quite) in the two points of the popular termini; - not quite, I say, because, from the coincidence of $\mathrm{M}$ and $\mathrm{D}$ in ${ }^{2}$, the popular law of progression must be altered from what it has previously been, as is quite manifest, for after $\mathrm{M}$ and $\mathrm{D}$ coincide, $\mathrm{C}$ and $\mathrm{D}$ must necessarily thereafter progress by the same ratio; so also must $M$ and $B, \& c$. ; and a mean between $M$ and $C$ thenceforward will be $M$ of twice the sides; besides, if $\mathrm{M}$ and $\mathrm{D}$ descend by the same ratio, they will converge towards each other, and hence, on this account alone, will place the conceived termini of the final $M$ above the popular place. This is a portion of the subject that may be exemplified to great extent, but I forbear.

$\mathrm{Y}$ is the constant mean between $\mathrm{C}$ and $\mathrm{D}$, and is the farther of three means from $\mathrm{A}$ to $\underset{ }{2}$; hence, when $\mathrm{Y}$ is in $\underset{ }{2}, \mathrm{~A}$ is also in 2 , and consequently, $\mathrm{B}$ is in 2 .

$\mathrm{Z}$ is the constant mean between $\mathrm{M}$ and $\mathrm{D}$, and between $\mathrm{C}$ and $\mathrm{B}$, and when $\mathrm{M}$ and $\mathrm{D}$ are in $2, \mathrm{Z}$ is a mean between them; or when $Z$ is in $2, M$ and $\mathrm{D}$, as also the area of such major $p$ are in $\underset{2}{2}$. Thus when $\mathrm{Z}$ is in $\underbrace{2}$, it is $=\mathrm{M}$, or the area of such major $p$; and is $=\mathrm{D}$ of a like $p$. 
So when $\mathrm{Y}$ is in 2 , it is $=\mathrm{A}$ and $\mathrm{B}$ of $\operatorname{such} p$, for $\mathrm{Y}$ is the farther of three means from $\mathrm{A}$ to ${ }^{2}$; and $\mathrm{A}$ is $=\mathrm{C}$ of half the sides until $\mathrm{A}$ and $\mathrm{C}$ become $=$, namely, until they become $=\mathrm{M}$.

Hence $\mathrm{A}$ in ${ }^{2}$ is $=\mathrm{C}$, for $\mathrm{Y}$ cannot be $=\mathrm{C}$ of half the sides; and when $\mathrm{Y}$ is $=\mathrm{C}$, it is also $=\mathrm{D}$, for $\mathrm{Y}$ is a mean between $\mathrm{C}$ and $\mathrm{D}$. Hence $\mathrm{C}$ in 2 is $=\mathrm{M}$.

We shall all agree that if the polygonal denotations expressed by capital letters, be considered in the nature of flowing quantities, from the $p 4$, or rather from the $p 8$, preserving the same relative situation to each other, (viz. those descending and also those ascending,) until they commence forming coincidences in 2 , that $\mathrm{Y}$ and $\mathrm{Z}$ will coincide at the same time (if not at the same place) that $\mathrm{M}$ and $\mathrm{C}$ do.

We shall all agree that the square of $\mathrm{Z}$ is always a mean between .5 and $\mathrm{M}$, and that the square of $\mathrm{Y}$ is always a mean between .5 and $\mathrm{A}$; consequently, when the square of $\mathrm{Y}$ becomes a mean between .5 and $\mathrm{C}, \mathrm{C}$ is $=\mathrm{M}$; and if such be in ${ }^{2}$, then all the denotations will coincide together in 2 . Hence the place of the coincidence of $\mathrm{Y}$ and $\mathrm{Z}$ is important. $\mathrm{Y}$ commences from $\mathrm{A}$ of the $p 8$; and $\mathrm{Z}$, from $\mathrm{B}$ of the $p 8$, and the popular conclusion is, that they coincide $\frac{1}{6}$ below 2 , that $\mathrm{A}, \mathrm{C}$ and $\mathrm{M}$ coincide $\frac{2}{3}$ below $\underbrace{2}$, that $\mathrm{B}$ and $\mathrm{D}$ coincide $\frac{1}{3}$ above ${ }^{2}$ and $\mathrm{E}, \mathrm{F}$ and $\mathrm{G}_{\frac{4}{3}}$ above 2 . So the popular conclusion is, that the denotations on their passage to their final coincidences, often salute each other and pass on; thus M, Z, D and G coincide in $\underset{2}{2}$, but each is destined to pass on in order to form other coincidences. So also M, and B of twice the sides, (according to the popular method, will coincide $\frac{2}{3}$ above 2 , or the adverse of the popular coincidence of $\mathrm{M}$ and $\mathrm{C}$, with others formed, and again dispersed in the vicinity of $\underbrace{2}$. But, by the popular mode, not a single final coincidence is in 2. Nevertheless, if $\mathrm{Y}$ and $\mathrm{Z}$ were to coincide in $\underset{2}{2}$, all the other denotations would coincide in $\underset{2}{2}$. Hence, if either denotation be final in ${ }^{2}$, all the denotations would coincide in ${ }^{2}$; in which case the capacities of the linear measures of the circle will be the same as those of the $p 4$, and that they are so, is certainly sustained by too much evidence, both general and special, to be doubted or denied.

Our principal object is, to ascertain the point in which $\mathrm{C}$ shall become $=\mathrm{A}$, or when $\mathrm{C}$ shall become $=\mathrm{M}$, viz. when all surrounding area shall be excluded from $\mathrm{A}$, of the circle, by the periphery; or perhaps, after the manner of Leibnitz, that numeral position in which the curve of the circle shall osculate all the angles contained as well in the perimeter of the circumscribed, as of the inscribed polygon. That is, when a mean between $\mathbf{Y}$ and $\mathrm{Z}$, (which, as in other cases, is always $\mathrm{Y}$ of twice the sides,) 
becomes extinguished by the coincidence of $\mathrm{Y}$ and $\mathrm{Z}$, the square of either $\mathrm{Y}$ or $\mathrm{Z}$ will be a mean between .5 and the osculating point, or point in which $\mathrm{C}$ becomes $=\mathrm{M}$, or is merged in $\mathrm{M}$. If then $\mathrm{Y}$ final be in 2 , its square is a mean between .5 and $\mathrm{A}$, which is then $=\mathrm{M}$; its square is also a mean between .5 and $\mathrm{C}$, which is then $=\mathrm{M}$.

A polygonal progression, then, does not (as has been supposed,) depend upon an alternate succession of equal and rational means; but, on the contrary, from the $p 8$ onward to the circle, namely, throughout the whole progression through the secondary polygons of the series, it proceeds wholly by rational means; in which, what may be called a denotation of twice the sides of its fellows is always a mean between them. Thus, $b a 8$ is a mean between $b a 4$ and $d a 4 ; b a 16$ is a mean between $b a 8$ and $d a 8$, and so on, $a d$ infinitum. So $b a 8$ is $=b 4$, when $m 4$ is 1 , and so on to a coincidence in the third or cube root of 2 . So a mean between $\mathbf{E}$ and $\mathbf{G}$ is $\mathbf{E}$ of twice the sides, unto a coincidence of $\mathrm{E}$ and $\mathrm{G}$; a mean between $\mathrm{B}$ and $\mathrm{D}$ is $\mathrm{B}$ of twice the sides, ad infinitum, and the like is the case in respect to $\mathrm{Y}$ and $\mathrm{Z}$.

Such being the premises, the conclusion need not be mistaken.

As a kind of synopsis to the quadrature, (upon which too much evidence cannot well be adduced,) it may not be improper to offer it in a dress or version somewhat new.

Thus $b a$ may denote the point in which $b$ and the area of any given $p$ are $=; d a$ may denote the point in which $d$ and the area of a $p$ are $=; M a$ may denote the point in which $m$ and the area of a $p$ are $=$; (whioh point has heretofore been denoted by M.) $\mathbf{C}$ denotes a mean between $\mathrm{M} a$ and $\mathrm{A}$.

A mean between $b a$ and $d a$ of any given $p$ is $=b a$ of a $p$ of twice the number of sides; hence, a mean between $b a$ and $d a$ of any given $p$, is the reciprocal of a mean between $\mathrm{A}$ and M $a$ of a like $p$.

$B$ is the farther of three means from $E$, or half the square of $b a$ to 2 , and $D$ is the farther of three means from G, or half the square of $d a$ to $2 ; \mathrm{Y}$ is the farther of three means from $\mathrm{A}$ to ${ }^{2}$, and is a mean between. $\mathrm{C}$ and $\mathrm{D}$; and $\mathrm{Z}$ is the farther of three means from $M a$ to $\underbrace{2}$, and is a mean between $M a$ and $\mathrm{D}$.

Thus, a mean between $d a$ and $b a$ is $b a$ of a $p$ of twice the sides, until such mean between $d a$ and $b a$ no longer exists, or until $b a$ is merged and extinguished in $d a$ of the circle, namely, until there is no longer $d a$ or $b a$ of a $p$ of sides.

When $d a$ and $b a$ coincide, it is manifest that $\mathbf{A}$ and $\mathbf{C}$ be- 
come merged and extinguished in $\mathbf{M} a$ of the circle; for $\mathrm{A}$ is the reciprocal of $b a$, and $\mathrm{C}$ is the reciprocal of a mean between $b a$ and $d a$. And the point in which $b a$ becomes merged in $\mathrm{D} a$, is the reciprocal of the point in which $\mathrm{A}$ and $\mathrm{C}$ become merged in $M a$ by any system.

$\mathrm{A}$ mean between $\mathrm{E}$ and $\mathrm{G}$ is $=\mathrm{E}$ of twice the sides, until $\mathrm{E}$ becomes merged in $\mathrm{G}$. $\mathrm{A}$ mean between $\mathrm{B}$ and $\mathrm{D}$ is $=\mathrm{B}$ of twice the sides, until $B$ is merged in $D$. And a mean between $\mathrm{Y}$ and $\mathrm{Z}$ is $=\mathrm{Y}$ of twice the sides, until $\mathrm{Y}$ is merged in $\mathrm{Z}$.

Hence, we may very properly consider, that on arriving at the quantities and measures of the circle, all means between similar denotations are extinguished, or do not exist. Hence, that in the quantities or measures of the circle, there is neither $b a, \mathrm{E}, \mathrm{B}$, $\mathrm{A}, \mathrm{C}$, or $\mathrm{Y}$, as all those (while they exist) denote polygons of sides.

Nevertheless, in the final event, the only proper quantities and measures of the circle, for which we are seeking, are those expressed by $\mathrm{M} a$ and $\mathrm{D}$; and the progressive ratios of $\mathbf{M} a$ and $\mathrm{D}$ are found, by any system, to coincide in 2 .

The object of research, however, is to ascertain the point in which $b a$ becomes extinguished in $d a$, for in the reciprocal of such point $\mathrm{A}$ becomes merged or extinguished in $\mathrm{M} a$; and in a like $p$, namely, in the circle, $\mathrm{B}$ becomes extinguished in $\mathrm{D}, \mathrm{Y}$ in $\mathrm{Z}$, and $\mathrm{E}$ in $\mathrm{G}$.

So long as $b a$ exists, we necessarily conceive of a mean betureen $b a$ and $d a$; and the like is the case in respect to $\mathbf{E}$ and $\mathrm{G}, \mathrm{B}$ and $\mathrm{D}, \mathrm{A}$ and $\mathrm{M} a$, or $\mathrm{Y}$ and $\mathrm{Z}$. Hence, we may as well conceive that $d a$ coincides with $b a$ of twice the sides, as with $b a$ of a like number of sides with $d a$; for $d a$ forms a coincidence as much with one as with the other. Hence, if we say that $d a$ coincides with $b a$ of twice the sides, it is also a coincidence with $b a$ of the same number of sides with $d a$, (if we may speak of number where there is no number,) and the like is true in respect to $\mathrm{D}$ and $\mathrm{B}$, of $\mathrm{M} a$ and $\mathrm{A}$, of $\mathrm{G}$ and $\mathrm{E}$, or $\mathrm{Z}$ and $\mathrm{Y}$.

Now $\mathrm{A} 8$ is 2 below half the square of $d a 8$, and $\mathrm{M} a 8$ is 2 below half the square of $b a$ of twice the sides; and such is, by the popular determination, conceived to be the relative situation of $\mathrm{A}$ and $\mathrm{M} a$, onward to the circle, in which case such 22 is supposed constantly to increase in value.

We have retained, then, as measures or quantities of the circle, (after the extinguishment of $\mathbf{E}, \mathbf{B}, \mathbf{C}, \mathbf{A}$ and $\mathrm{Y}$ ) those of $\mathrm{M} a, \mathrm{D}, \mathrm{Z}$ and $\mathrm{G}$; and, by the popular method of considering the subject, as well as by any other method that may be de- 
vised, those four proper measures, or quantities, of the circle, coincide together in $\underbrace{2}$. That is, all the proper measures of the circle, situated below unity, necessarily coincide together in $\underbrace{2}$; hence, if either proper measure of the circle is in $\underset{2}{2}$, the others are also.

But again, $\mathrm{C}$, or a mean between $\mathrm{A}$ and $\mathrm{M} a$, coincides with $\mathrm{B}$ of twice the sides of such $\mathrm{M} a$; and we also say that $\mathrm{D}$ coincides with $B$ of twice the sides. But, by the popular method, one of these coincidences would be in 2 , while the other would be $\frac{1}{3}$ above 2 . So $\mathrm{M} a, \mathrm{D}, \mathrm{Z}$ and $\mathrm{G}$, (the proper measures of the circle,) coincide in ${ }^{2}$, and $\mathrm{A}, \mathrm{B}, \mathrm{Y}$ and $\mathrm{E}$, (extinguishable quantities,) are conceived to coincide together in $\underbrace{2}$. But $A, B$, $\mathrm{Y}$ and $\mathrm{E}$ are as well means as principals ; and hence, $\mathrm{M} a, \mathrm{D}, \mathrm{Z}$ and $G$ will coincide in $2 ; A, B, Y$ and $E$ will coincide in 2 ; and $a$ mean between $\mathrm{A}$ and $\mathrm{M} a$, a mean between $\mathrm{B}$ and $\mathrm{D}, \mathrm{a}$ mean between $\mathrm{E}$ and $\mathrm{G}$, and a mean between $\mathrm{Y}$ and $\mathrm{Z}$, coincide together in 2 ; and this upon the simple principles of polygonal progression, which all may readily examine, and of the truth of which all may satisfy themselves; and such phenomena cannot exist, unless 2 be the point in which the proper measures of the circle coincide, and in which all other polygonal quantities cease to exist.

Nevertheless, when we consider 2 as the point in which a mean between $A$ and $M a$, between $B$ and $D$, between $E$ and $G$, and between. $\mathrm{Y}$ and $\mathrm{Z}$ coincide, we necessarily, in our conception, transpose the terms of half the sides, and imagine those which have previously been above 2 to have passed below 2 , and those which have been previously below 2 to have passed above 2 ; which necessarily arises from our conception, that there is a progression so long as we retain the terms denoting polygons of sides.

Thus, if we conceive such means to coincide in 2 , that is, if we conceive $\mathrm{C}$, as a mean between $\mathrm{A}$ and $\mathrm{M} a$, to be in $2, \mathrm{M} a$ will be conceived to occupy the identical place that $\mathrm{A}$ does, when $M a$ is in 2 ; and $A$ will appear to occupy the place of $\mathrm{M} a$ of half the sides of $\mathrm{M} a$ in ${ }^{2} ; \mathrm{B}$ will be in the place of $\mathrm{C}$, when $M a$ is in 2 ; and $D$ will be in the place of $B$, when $M a$ is in 2 , \&c. But this is but conception, founded upon that which still conceives the progression to be continued; which, if true, would place $\mathrm{M} a$ below $\mathrm{A}$ or $\mathrm{C}, \mathrm{B}$ below $\mathrm{D}, \mathrm{E}$ below $G$, and $Z$ below $Y$; and such a phenomena no one expects to see.

When $\mathrm{M} a$ and $\mathrm{D}$ are in ${ }^{2}$, such $\mathrm{M} a$ or $\mathrm{D}$ is half the square of $d a$; and when $\mathrm{A}$ and $\mathrm{B}$ are in $\underbrace{2}$, such $\mathrm{A}$ or $\mathrm{B}$ will be half the square of $b a$. 
And if we conceive $\mathrm{C}$ to be in 2 , namely, a mean between $\mathrm{A}$ and $\mathrm{M} a$, such $\mathrm{C}$ will be conceived to be half the square of a mean between $d a$ and $b a$; in which case, such $d a$. will be conceived to be above $b a$, being an event which cannot occur.

Let us suppose 2 to be the termini of an infinite series, for the progressive ratios of $\mathrm{M} a$ and $\mathrm{D}$, and of $\mathrm{A}$ and $\mathrm{B} ; \mathrm{B} 4$ and $\mathrm{M} a 4$ are in $1, \mathrm{D} 4$ in ' 3 , and $\mathrm{A} 4$ in $\underbrace{6}$. If we progress' by successive means, between M $a 4$ and 2 , and between $\mathrm{D} 4$ and ${ }_{2}^{2}$, the termini will be in $\underset{2}{2}$; or if we proceed, by successive means, between B 4 and 2 and between $A 4$ and 2 , the termini will also be in 2 . But in such case, we conceive the last step in the progression, - from D 4 to $2,-$ to be one step in advance of a like number of steps, in the progression from A 4 to 2. That is, in the coincidence of D with ${ }_{2}^{2}$, we conceive $A$ to be one step behind or below 2 , which step we conceive to be 2 below 2 . And hence we conceive, that when $\mathrm{D}$ (and consequently $\mathrm{M} a$ ) is in ${ }^{2}, \mathrm{~B}$ of the same number of sides is 1 above 2 ; being thus transported, by our conception, from the fact, that in proceeding from $\mathrm{M} a 4$, or from $\mathrm{B} 4$, we have progressed or travelled in the same, or in the identical footsteps in both cases, and also by the same number of footsteps. And in such progression, - from M $a 4$ to $\underbrace{2}$ and from D 4 to $\underbrace{2}$, or from B 4 to ${ }_{2}^{2}$ and from $A 4$ to ${ }_{3}^{2}$, - the results will be the same, whether we progress by a constant mean or by the farther of two, the farther of three, and so on, ad infinitum, until numbers or means are exhausted, or to an actual coincidence. Nevertheless, the most natural method, and at the same time that affording the most ready explanation and evidence, is probably that proceeding by the farther of two means.

In such case, (as by any other progression,) when the progressive ratios of $M a$ and $D$ coincide in $\underset{2}{2}$, we conceive $A$ to be $\underline{2}$ below 2 , and $\mathrm{B} 1 \frac{1}{2}$ above 2 ; nevertheless, we have passed or progressed by the same steps, in respect to $\mathrm{B}$ as to $\mathrm{M} a$, from their coincidence in the $p 4$, and hence the farther of two means arrives as soon in 2 in one case as in the other.

And to aid us in a proper conception of the matter, the economy of numbers, and that of quantities, are so curiously adjusted to each other, that we need not remain in much doubt; for if we conceive another step of the progression in respect to A and B, we shall find $A$ and $B$ in their vanishing points according to the popular determination, - that is, in the points in which they become merged or extinguished in $\mathrm{M} a$ and $\mathrm{D}$ of the circle; for by such additional step, we conceive $A$ to be $\frac{2}{3}$ below $\underset{2}{2}$, and B $\frac{1}{3}$ above 2 , or in the popular $\mathrm{M} a$ and $\mathrm{D}$ of the circle.

The utmost then, which can be claimed, is, that when $M a$ 
and $\mathrm{D}$ coincide, the very next step in the progression, applied to $A$ and B, extinguishes them. And the same remark applies to $\mathbf{Y}$ and to $\mathrm{E}$, for by such progression, when $\mathbf{M} a$ and $\mathbf{D}$ are in 2 , (and consequently $Z$ and $G$,) the very next step in the progression, applied to $\mathbf{Y}$ or to $\mathrm{E}$, places them in $\mathrm{Z}$ and $\mathrm{G}$ final, according to the popular notion of their final positions.

And because $\mathrm{G}$ and $\mathrm{Z}$ are in 2 when $\mathrm{M} a$ and $\mathrm{D}$ are contained in it, and because $\mathbf{E}$ and $\mathrm{Y}$ coincide with $\mathrm{A}$ and $\mathrm{B}$ in $\underset{2}{2}$, and are extinguished in the same $p$ as are A and B in our present consideration of the subject, we may dispense with $G$ and $Z$, as also with $\mathrm{E}$ and $\mathrm{Y}$, and merely consider $\mathrm{C}$ as a mean between $A$ and $\mathbf{M} a$. Thus in the foregoing example of a progression from $\mathrm{M} a$ or $\mathrm{B}$ of the $p 4$, or from $\mathrm{A}$ and from $\mathrm{D}$ of the $p 4$, if we adopt a simple mean in lieu of the farther of two means, then when $M a$ and $D$ are in $\underset{G}{2}$, if we conceive $A$ and $B$ to take another step in the progression, we shall conceive it to place A 1 below 2 , and B $\frac{1}{2}$ above ${ }_{2}^{2}$. But if, when $\mathrm{M} a$ is in $\underset{2}{2}$, the quantity denoted by 1 is already extinguished, except in the imagination or conception, then $\mathrm{A}$ and $\mathrm{B}$ are in $\underbrace{2}$ when $\mathrm{M} a$ and $D$ are; in which case, such $A$ and $B$ of twice the sides will only be in their respective vanishing means.

When either $\mathrm{B}$ or $\mathrm{D}$ coincide with a mean between $\mathrm{B}$ and $\mathrm{D}$, it is but the coincidence of $\mathrm{B}$ and $\mathrm{D}$, or rather the extinguishment of $B$ as well as that of a mean between $B$ and $D$; notwithstanding we may still conceive both $B$ and such mean to exist; and the like is true in respect to $\mathrm{A}$, and $\mathrm{M} a, \& c$. , in which case, even such extinguished mean will be conceived to be $\mathrm{B}$ or $\mathrm{A}$ of twice the sides; for from the $p 8$ onward, $\mathrm{B}$ is but a mean between $B$ and $D$ of half the sides, and $A$ is but a mean between $A$ and M $a$ of half the sides; and when those means are extinguished, $\mathrm{M} a$ and $\mathrm{D}$ only remain as proper quantities of the circle. Hence, as $\mathrm{C}$ is but a mean between $\mathrm{A}$ and $\mathrm{M} a$, when such mean is extinguished, $\mathrm{C}$ is also extinguished, or is merged in $\mathrm{M} a$.

Thus the extinguishment of $A$ and $B$ is the extinguishment of flowing polygonal quantities, or rather of quantities denoting polygons of sides; and we must conceive them to flow until those quantities which necessarily denote polygons of sides are extinguished, and until we can have no conception of a farther progression, or until any farther attempt at a progression will apparently cause a retrograde movement.

Now the progressive ratios of $\mathrm{A}$ and $\mathrm{B}$ coincide in $\underset{2}{2}$, by any system, and if 2 be the point of their extinguishment, it is then the point of a general coincidence; and it is also clear, that we cannot conceive an extension of the progression beyond the coincidence of $\mathrm{A}$ and $\mathrm{B}$, for an attempt at a farther progression 
gives an apparent retrograde movement, and transposition of terms.

Thus, if $\mathrm{A}$ and $\mathrm{B}$ coincide in $\underbrace{2}$, we conceive $\mathbf{M} a$ of a like. $p$ to be $\frac{1}{2}$ below 2 (going upon the ground that from the $p 8$; or even from the $p 4, \mathrm{M} a$ shall be constantly denoted by 1 below B.) But A in ${ }^{2}$, as in any other case, is a mean between $A$ and $M a$ of half the sides; or, as has all along been considered, is $=\mathrm{C}$ of half the sides.

C in 2 , then, denotes a $p$ of half the sides of that whose $A$ is in 2 , and $\mathrm{C}$ is a mean between $\mathrm{A}$ and $\mathrm{M} a$ of the $p$ which it denotes, and $\mathrm{C}$ in ${ }_{2}^{2}$ denotes a $p$ whose $\mathrm{M} a$ is 2 below 2 , and whose $\mathrm{A}$ is 2 above $\underset{2}{2}$, (occupying the identical places of $M a$ and $\mathrm{C}$ of the $p$ of half the sides of that whose $\mathrm{M} a$ and $\mathrm{D}$ are in 2. .)

Thus where A and B coincide, (or appear to,) we cannot conceive of a $p$ of twice the sides, for polygonal quantities denoting sides, are then extinguished, and if we conceive a $p$ of half the sides, (namely, the $p$ whose $C$ is conceived to be in ${ }^{2}$,) we find $A$ to be -2 above 2 , namely, $\underline{2}$ above $A$ of twice the sides; which phenomenon will not be required by any one.

Thus the final or extinguished mean between $\mathbf{A}$ and $\mathbf{M} a$, and the final or extinguished mean between $B$ and $D$ coincide in 2 ; and until such apparent coincidence, we may conceive a continuance of the progression.

Then considering $\mathrm{C}$ to denote simply a mean between $\mathrm{A}$ and $\mathrm{M} a$, when $\mathbf{C}$ is conceived to be in $\underset{2}{2}$, it is but the point of its extinguishment; namely, the point of extinguishment between $A$ and $M a$. Hence, in a general consideration of the quantity denoted by $\mathbf{C}$, its more proper consideration is that of simply a mean between $\mathbf{A}$ and $\mathbf{M} a$; nevertheless, its consequence is vastly important when considered as one of the prime linear quantities.

Our utmost researches then, are not only extended to extinguished means in which certain approximating quantities coineide, but also to the general coincidence of those extinguished means ; which general coincidence is in 2 , by any rational progression that may be devised. And then, and then only, do we find the capacities of polygonal measures to again become the same as they were in the $p 4$, and hence to be capable of a rational application.

I have before remarked upon the allegation, which we often find repeated by eminent geometers and mathematicians, that a polygon is, properly speaking, the area, and not its linear measures, as is often supposed; but I have been rather inclined to adopt the vulgar, or common acceptation, believing it to be 
best suited to the subject, if but one is to be adopted. Nevertheless, with a view to a full consideration of the subject, the areas should be considered in connection with their linear measures; at least so far that, whenever linear measures of polygons are to be compared and proportioned to each other, each linear measure shall denote or express the amount of area of the polygon whose linear measure is given, or of a polygon of half the sides, or of twice the sides; thus $\mathrm{B}$ or $\mathrm{D}$ always denotes the area to be $.5 ; \mathrm{M} a$ or $\mathrm{M}$ denotes either the area, or one-fourth of the circumference of a major polygon; while $\mathrm{C}$ denotes one fourth of the circumference of a minor polygon, and also the area of a minor polygon of twice the sides.

Hence, by the use of $M, C, B$ and D we have as full and as proper an expression or denotation of the areas of the major and minor polygons, as also of all primal polygons, at least from the octagon, (or polygon from which our equation commences,) as though the areas themselves were expressed.

But the converse of this could not occur, and more especially in respect to diametric quantities of polygons ; for each polygon of sides, while it has but one perimeter, has two diametric quantities, which must be considered in our investigations; hence, while by means of the linear measures we may denote the quantities of all necessary areas, we cannot, by means of the areas, denote all the necessary linear measures.

And to show that a direct use of areas may be dispensed with, and that we may conduct our progression wholly by linear quantities, it is only necessary to allege the fact, that a mean between $\mathrm{A}$ and $\mathrm{B}$ of any given $p$, is also a mean between $\mathrm{C}$ and $\mathrm{D}$ of the same $p$; and hence, when $\mathrm{A}$ and $\mathrm{B}$ shall coincide, it will be as well in the mean point between $C$ and $D$, as between $A$ and $B$. So also, when $C$ and $D$ coincide, it must be in the mean point between $\mathrm{A}$ and $\mathrm{B}$ of the same $p$; and hence we may, in general, adopt $\mathrm{C}$ and $\mathrm{D}$ in our progression, to the exclusion of $\mathrm{A}$ and $\mathrm{B}$.

So a mean between $\mathrm{M}$ (or $\mathrm{M} a$ ) and $\mathrm{D}$ is also a mean between $\mathrm{C}$ and $\mathrm{B}$ of the same $p$; hence, when $\mathrm{M}$ and $\mathrm{D}$ coincide, it as well in the mean point between $C$ and $B$, as between $M$ and $\mathrm{D}$; hence, when $\mathrm{C}$ and $\mathrm{B}$ shall coincide, it must, of necessity, be in the mean point between $M$ and $D$; hence, in our progression we may, in general, adopt $M$ and $D$, to the neglect of $C$ and $B,-$ regarding, however, the important fact, that $C$ is to $D$ of any given $p$ as $\mathrm{M}$ is to $\mathrm{B}$.

Then the farther of three means from $\mathrm{M}$ to ${ }^{2}$, (which is a mean between $M$ and $D$, ) will be the mean place of $M$ of twice the sides; and the farther of three means from $\mathrm{C}$ to $\underset{\mathrm{Z}}{\mathrm{C}}$, (which is a 
mean between $\mathrm{C}$ and $\mathrm{D}$ of twice the sides,) will be the mean place of $\mathrm{C}$, or of $\mathrm{D}$ of twice the sides.

Then, when $M$ is in 2 , it will be $=$ the mean place of $M$ of twice the sides; and when $\mathrm{C}$ is in $\underset{2}{2}$, it will be $=$ the mean place of $\mathrm{C}$ of twice the sides. The consequence is, that when $\mathrm{A}$ is in 2 , such $\mathrm{A}$ is then $=$ the mean place of $\mathrm{C}$ of the same $p$.

It is, then, equally well to speak of the true and mean places of $\mathrm{C}$ and of $\mathrm{M}$, as of $\mathrm{A}$, or of the area of a major $p$; nevertheless, if we adopt $M a$ in lieu of $M$, we then make the application of the true and mean places, as well to the area as to $\mathrm{M}$.

But, in respect to minor polygons, $\mathrm{C}$ is $=\mathrm{A}$. of twice the sides; nevertheless, the farther of three means from $A$ is the mean place of $\mathrm{A}$ of twice the sides, and the farther of three means from $\mathrm{C}$ is the mean place of $\mathrm{C}$ of twice the sides, until the progression is ended.

Thus the true and mean place of A 8 is the same, while the true place of $\mathrm{M} a 8$ is 1 below the mean place ; so also, the true place of $\mathrm{C} 8$, or of $\mathrm{A} 16$, is $\frac{1}{2}$ below the mean place.

$\mathrm{Y}$, then, may denote the mean place of $\mathrm{C}$ and of $\mathrm{D}$, and $\mathrm{Z}$ may denote the mean place of $\mathrm{M} a$ of twice the sides, or of $\mathrm{B}$ of twice the sides; thus B 8 is as well in its mean place as in its true place; and such construction, in respect to the true and mean places of $\mathrm{M} a, \mathrm{~B}, \mathrm{C}$ and $\mathrm{D}$ are not only in perfect aecordance with the law of polygonal progression, but the same is even corroborated by the popular method.

Thus $\mathrm{C}$ is, proportionally, half as far below the mean place of $\mathrm{C}$ or $\mathrm{D}$, as $\mathrm{M} a$ is below the mean place of $\mathrm{M} a$ or $\mathrm{B}$; and $\mathrm{C}$ is proportionally as far too low, in respect to the mean place of $D$, as in respect to the mean place of $\mathrm{B}$.

Now, while the true place of $\mathrm{C} 8$ is a mean between the true place of A 8 and the true place of $\mathrm{M} a$, so the mean place of $\mathrm{C} 8$ is a mean between the mean place of $\mathrm{A} 8$ and the mean place of M $a 8$; and the like principle holds throughout the progression. Or thus, the mean place of $\mathrm{C}$ is a mean between the mean place of $\mathrm{A}$ and the mean place of $\mathrm{M} a$, or of $\mathrm{B}$.

When $A$ is in 2 , such $A$ is in the mean place of $C$ of the same $p$; hence such $\mathrm{A}$ (as the mean place of $\mathrm{C}$ ) is a mean between the mean place of such $\mathrm{A}$ and the mean place of $\mathrm{M} a$, or $\mathrm{B}$.

So $A$ in 2 is a mean between its mean place and the mean place of $\mathrm{M} a$, or $\mathrm{B}$; $\mathrm{A}$ in ${ }^{2}$ is in the mean place of $\mathrm{C}$, or of $\mathrm{D}$; and $\mathrm{C}$ in 2 is in the mean place of $\mathrm{C}$, or of $\mathrm{D}$ of twice the sides. And the like is true of $\mathrm{B}$ in $\underset{C}{2}$ as in the case of $\mathrm{A}$; so $\mathrm{M} a$ and 
$\mathrm{D}$ in ${ }^{2}$ are in the mean place of $\mathrm{C}$ and $\mathrm{B}$ of the same $p$, or of $\mathrm{M} a$ and $\mathrm{B}$ of a $\boldsymbol{p}$ of twice the sides.

Thus infinite wisdom, which has farnished geometry and mathematics with the best of reasons, but which never intended that we should survey a field or measure and determine the contents of an area, without taking into the account both the circumferential and diametric measures of such area, has, in the present case, - as if to invite us to quadrate our ways at once, and not to be eternally attempting to equate them to some supposed, but erroneous, rule or law, - so contrived and adapted the evidence, as not only to show us the necessity of an equation of the varying capacities of polygonal measures, as we progress from the square to its inscribed circle, but also to dictate, in divers ways, the manner of conducting the same. The neeessity of this equation can only be detected, by a comparison of the perimetric and diametric quantities of polygons; nor can the equation be made but by some law of progression, by which those quantities are constantly compared with each other.

$\mathrm{C}$ is to $\mathrm{D}$ as $\mathrm{M} a$ is to $\mathrm{B}$; and this is the form in which the progression proceeds, from the trigon to the circle ; nevertheless, in the $p 4$ the relative situations of $C$ and $D$ are changed, as is also the case in respect to $\mathrm{M} a$ and $\mathrm{B} ; \mathrm{C}$ and $\mathrm{D}$ being equal in the $p 4$, and $\mathrm{M} a$ and $\mathrm{B}$ being equal in the $p 4$. But the $p$ 8 presents the variance between $C$ and $D$, or between $M a$ and $B$, which $_{9}$ is denoted by $\frac{1}{\text {, and which requires to be equated to } 0}$ in the circle, in lieu of being increased in value as we progress from the $p 8$ to the circle, as is the case by the popular determination.

It may not, however, be surprising, that the mind should not readily assent to an equation of the difference existing between $\mathrm{M} a$ and $\mathrm{B}$ (and consequently between $\mathrm{C}$ and $\mathrm{D}$, ) of the $p 8$ to $\underline{0}$, on arriving at 2 , but rather that it should conceive the same ratio to exist, as we progress onward from the $p 8$; and hence, when the progressive ratio of $M a$ is in 2 , that the same ratio exists between $\mathrm{M} a$ and $\mathrm{B}$ as existed in the $p 8$; and hence, the actual numerical difference between $\mathrm{M} a$ and $\mathrm{B}$, when $\mathrm{M} a$ is in ${ }^{2}$, would be but very little less than it is in the $p 8$. And such would be the case, were the capacities of the linear measures of all the polygons situated between the $p 8$ and circle, the same as they are in the $p 8$; but not so if, from the $p 8$ towards the circle, the capacities of the measures of polygons are again approximating to what they were in the $p 4$, from whence we commenced our progression.

But having found in the $p 8$ (or in the first step in the progression,) a difference between $\mathrm{M} a$ and $\mathrm{B}$, or between $\mathrm{C}$ and $\mathrm{D}$, 
and finding the law to be universal, that $\mathrm{M} a$ is to $\mathrm{B}$ as $\mathrm{C}$ is to $\mathrm{D}$, and, consequently, that $\mathrm{C}$ is to $\mathrm{M} a$ as $\mathrm{D}$ is to $\mathrm{B}$; the mind which is imbued with the least tincture of ratio and proportion will naturally attach to any sign, denoting such difference in the $p 8$, the conception that it is a sign denoting ratio and proportion, and that, as such, it will accompany the polygonal progression, and that it will constantly be denoted by the same character or sign as is used for the $p 8$, which, if it were simply to denote ratio, would only become extinguished in 0 ; whereas, by a proper equation of the capacities of polygonal quantities, it necessarily becomes extinguished in the circle.

The consequence of such natural or rational conception of the mind is, that when $M a$ and $D$ (which are in advance of their mean places from the $p 8$ onward) coincide in ${ }^{2}$, or in their mean places, $\mathrm{B}$ is still conceived to be 1 above $\mathrm{M} a$, and $\mathrm{C}$ 1 below $\mathrm{D}$; thus conceiving the difference of $\underline{2}$ between $\mathrm{C}$ and $\overline{\mathrm{B}}$, notwithstanding they actually coincide with $\mathbf{M} a$ and $\mathrm{D}$ in 2 .

Such conception, however, is not unprofitable, as it enables us to give (in a formula, if we please) the relative situations of the polygonal quantities and measures, not only of the $p$ whose $\mathrm{M} a$ and $\mathrm{D}$ coincide in 2 ; but also of the $p$ of half the sides, or of twice sides; for it will be recollected that the circle is composed of a $p$ of half the sides and a $p$ of twice the sides, as well from the minor as from the major polygons; which phenomenon is the result of a demonstration, the truth of which is readily perceived.

It may not be inappropriate here, to suggest something farther in reference to the measures and quantities of the $p 8$; and of the important positions which they hold in polygonal economy. Thus, the true places of $\mathrm{A}$ and of $\mathrm{B}$ of the $p 8$, are also their mean places, and A 8 is the square of $B 8$. And while $B 8$ is a mean between $\mathrm{B}$ of any given $p$, and the square root of $\mathrm{A}$ of such $p$; or between $\mathrm{D}$ of any given $p$ and the square root of $\mathrm{M} a$ of such $p, \mathrm{~A} 8$ is a mean between $\mathrm{B}$ and the square root of $\mathrm{Y}$ of any given $p$, viz. between $\mathrm{B}$ of any given $p$, and the square of the mean place of $\mathrm{C}$ or $\mathrm{D}$ of such $p$, or of the mean place of $\mathbf{A}$ of a $p$ of twice the sides.

Hence, if B of a $p$ be in 2 , A S is the mean between such B and the square of the mean place of $C$ or of $D$, or of $A$ of twice the sides.

But $\mathrm{B}$ in 2 is = A, by any system, as also by the law of polygonal progression, and is a mean between $\mathrm{C}$ and $\mathrm{D}$ of the same $p,-$ or is in the mean place of $\mathrm{C}$ or $\mathrm{D}$ of the same $p,-$ and is consequently in the mean place of $\mathrm{A}$ of twice the sides; but, 
as I have before remarked, the place of $\mathrm{A}$ of twice the sides is nowhere to be found.

So we say that $\mathrm{A}$ in 2 is $=\mathrm{C}$ of half the sides ; consequently, C in 2 is = the mean place of $\mathrm{C}$ or of $\mathrm{D}$ of twice the sides, and $\mathrm{A}$ in 2 is $=$ the mean place of $\mathrm{A}$ of twice the sides. And because $M a$ in 2 is $=\mathrm{Z}$, hence, $M a$ in 2 is $=$ the mean place of $\mathbf{M} a$, or $\mathbf{B}$ of twice the sides.' The consequence is, that if we progress upon the mean place of $\mathrm{M} a$ and $\mathrm{B}$, and upon the mean place of $C$ and $D$, they will eventually coincide with the true places, and with each other. As the mean place of $\mathrm{M} a$ or of $\mathrm{B}$ is a mean between $M a$ and $\mathrm{D}$, or between $\mathrm{C}$ and $\mathrm{B},-$ namely, the farther of three means from M $a$ to ${ }_{2}^{2}$,- hence, if our progressive steps are upon the successive mean places of $\mathrm{M} a$ or $\mathrm{B}$, we can only arrive in 2 by an infinite series; and as a mean between $\mathrm{M} a$ and $\mathrm{D}$ is also a mean between $\mathrm{C}$ and $\mathrm{B}$, perhaps no one will contend that an infinite series will cause a coincidence of $\mathrm{M} a$ and $\mathrm{D}$ sooner than it will the coincidence of $\mathrm{C}$ and $\mathrm{B}$, or, that an infinite series of progressive steps will sooner extinguish a mean between $\mathrm{M} a$ and $\mathrm{D}$, than between $\mathrm{C}$ and $\mathrm{B}$.

Hence, $C$ and $B$ must coincide at the same time, and in the same point with $M a$ and $D$. And as a mean between $A$ and $B$ is also a mean between $\mathrm{C}$ and $\mathrm{D}$, an infinite approximation will cause $A$ and $B$ and $C$ and $D$ to coincide at the same time, and in the same point; and in respect to the coincidence of $A$ and $\mathrm{B}$, or of $\mathrm{M} a$ and $\mathrm{D}$, the same is in 2 .

But $\mathrm{M} a, \mathrm{D}, \mathrm{G}$ and $\mathrm{Z}$ finally abide, and denote the area and linear measures of the circle; and when they coincide together in 2 , (which occurs in accordance with any law of polygonal progression,) the true and mean places of $\mathrm{M} a$ coincide in 2 .

$\mathrm{A}, \mathrm{B}, \mathrm{E}$ and $\mathrm{Y}$ always denote polygons of sides, and hence are extinguishable quantities, or extinguishable means; for while they are considered in the nature of quantities, they are also considered as mean proportionals, and, whether as quantities or means, they are conceived to be progressing towards their final extinguishment, or to a merger in the abiding quantities; and hence, when they cease to exist as quantities, they also cease to exist as means; and by the law of progression, A, B, E and Y must apparently coincide in 2 ; and if either one of them is extinguished in $\underbrace{2}_{2}$, they all are; we nevertheless conceive them to exist (both as quantities and means) in the point of their extinguishment ; and hence we reject the conception of their final extinguishment, even in the abiding quantities which finally denote the circle; nor can such conception be relieved, except by the reductio ad absurdum. 


\section{SECTION FIFTH. - APPLICATION.}

In making a short application of my determination of the measures of the circle to that of solids, I shall go no farther than a short comparison with the prime sphere, namely, the sphere whose diameter is unity, and its circumscribed cylinder and prisms, amongst which I place the cube; nor need I go farther than to compare the prime sphere with its circumscribing cylinder and cube.

What is often termed the volume or contents of a solid, I shall call by the more appropriate name of bulk.

The surfaces of regular solids are as their bulks.

The bulk of the sphere is demonstrated to be two-thirds of the bulk of its circumscribing cylinder; and the surface of a sphere is $=$ to the lateral surface of its circumscribing cylinder.

The surface of the prime sphere, or of its circumscribing cylinder, or any circumscribing prism, is six times the bulk of the given solid.

The surface of a regular solid flows by a duplicate ratio, and the bulk by a triplicate ratio to that of any given linear measure of such solid.

The circumscribing cube of the prime sphere properly denotes unity amongst the solids, as the square does amongst the polygons.

The circumference of the prime sphere is $=$ the surface; hence, if the diameter of a sphere be expressed in a term of the prime table, any given power of the circumference will be expressed in terms of the table, - as also of the surface, but not of the bulk. Nevertheless, tables may be readily made upon the same ratio between terms of the table, as that of the prime table, in which the bulks of spheres may be expressed when their surfaces are expressed in the prime table, and in which the surfaces of cylinders and their bulks may also be expressed; and hence the whole may be thrown into rational and tabular form.

It is not my intention here (like Montucla) to enter into the sports of mathematics, nor to make sport of those things which I do not comprehend; but I have before suggested that number 8 , which has much to do with the octagon and its infinity of equal means, was not destitute of beauties; - it is the sum of all the powers of .8888 , ad infinitum, which is the square root of the revolving series .790123456790, ad infinitum, which series does not appear to have a drop of octagonal blood in it. And if either series be multiplied or divided by any natural number or definite 
quantity, the result will be a continuous, uniform, or revolving series, by which an infinity of equal means may be produced as exponential tables.

These revolving series are deduced from what may be termed the equivalents of the natural numbers in their order, of which I will give one or two examples in connection with a suggestion in respect to the law of falling bodies. And although I may hereafter give a more extended numerical formula in respect to the law of falling bodies, than the one $I$ here give, and one more fully showing the economy and adaptation of numbers to our every necessity, yet, perhaps one of the simplest formulas that may be given for the purpose is the following:

$$
\begin{array}{lllllllllllllll}
1 & & 1 & & 1 & & 1 & & 1 & & 1 & 1 & 1 & \\
1 & 2 & 3 & 4 & 5 & 6 & 7 & 8 & 9 & 10 & 11 & 12 & 13 & 14 & 15
\end{array}
$$

Thus if we take the natural numbers in their order, to the number fifteen, and divide them into periods for extracting their square root, making the figure 1 , at the left, the first period, the square root will come out in the constant figure 1 , over the last figure in each period, namely, over each odd number, making eight figures in such root, and the sum of all the odd numbers thus far will be 64 , or the square of the sum of all the figures in the root, and so on, ad infinitum, let the natural numbers be extended ever so far. And in the extraction of such root, the remainder will constantly come out in terms of the even numbers in their order, as $2,4,6,8,10,12,14$, \&c. Thus while each figure of the root denotes one moment of time, each odd number denotes the number of spaces passed over in each successive moment, and the successive even numbers denote the final velocity of the body at the end of each successive moment; and the total spaces passed over at the end of each successive moment, will be the square of the number of moments the body has been falling.

Hence, the whole space passed over is the square of the whole time of the fall; and as the force which urges the body in its fall is constant and uniform with the time, hence, in a falling body, the whole space passed over from the commencement of the fall, is as well the square of the whole force, as of the time, which is too important a matter to be overlooked in our investigations of the laws of force and motion, although I apprehend it has not always been sufficiently regarded.

This, however, is one of the numerical beauties of numbers, applicable to our wants, but we should not seek to extend it beyond its legitimate application.

But to show that this is a mere numerical dress of the law of falling bodies, I will present the natural numbers in their order, 
or what is equivalent thereto, in another dress or form, and which may be extended ad infinitum, and which will be found to have some application to the measures of the circle, and of the round bodies.

Thus, if we take the natural numbers in their order to any extent, and multiply the same by 2 , and then divide the product by 2 , we obtain a revolving series, ad infinitum, which may be said to be equivalent to the natural numbers in their order; which series will come out $1,2,3,4,5,6,7,9,0,1,2,3,4,5$, \&c., in which the figure 8 will never occur.

If we then divide such series into periods for the extraction of the square root, making figure 1 , at the left, one period, the root will continue to be expressed by figure 1 , over the last figure in each period, ad infinitum, and the remainder will constantly come out in terms of the even numbers, or what is equivalent thereto, namely, $2,4,6,9,1,3,5,8,0,2,4,6,9,1,3, \&$ c., which equivalent of the even numbers is produced in the same manner as that of all the natural numbers. So also an equivalent to the odd numbers is produced in the same manner, and will revolve thus: $1,3,5,8,0,2,4,6,9,1,3,5,8,0,2, \& c$. Which equivalents of the natural numbers in their order, when applied to the measures of the circle and of the round bodies, are not only useful, but their application is extremely beautiful; but they do not so well apply as formulas to express the law of falling bodies near the earth.

But to return to a farther application of the circle. When the diameter of the inscribed sphere of the cylinder is $=d$ of the circle whose $m$ is 1 , the bulk of such cylinder is the square of the diameter of the inscribed sphere. The circumference is four, and the surface will be six times the diameter of its inscribed sphere. But the proper diameter of the cylinder or cube, is the diameter of its inscribed sphere. If the diameter of the cylinder be the sixth root of six, its surface is six, and its bulk is = its diameter.

But this is the general law: wher the diameter of the sphere, cylinder or cube is the reciprocal of the bulk of a like prime solid, the bulk is the square of the diameter. Hence, when the diameter of the sphere is 1.889881 , or third root of 6.75 , the bulk is the square of the diameter, and the strface is six times the diameter, and the circumference is 6 . So when the diameter of such solid is the third root of the reciprocal of the bulk of a like prime solid, the bulk is 1 . The reciprocal of the bulk of the prime sphere is three times the square of $\mathrm{M}$ of the prime circle, or is three times three halves of $M G$ the prime circle, and the square of such reciprocal of the bulk of the prime sphere, is the third root of the square of $\mathrm{M} 3$. 
The square root of the reciprocal of the bulk of a prime solid is the point of equality between the bulk and diameter, and the third root of the square of such point of equality is the diameter of a like solid whose bulk is 1 ; and such point of equality in the prism of three lateral sides is = B 6 .

:888, ad infinitum, is six times the third power of the bulk of the prime sphere, and is = the area of the circle whose $m$ is $\overparen{2}$ above .666, ad infinitum, and is three halves of the bulk of the prism of three lateral sides, whose diameter is $=d 3$, when M 3 is $1, \& c$.

The reciprocal of the bulk of any prime solid is = the bulk of the circumscribing cube of a like solid whose bulk is 1 . But I will not pursue the subject farther; the object being simply to show the reciprocities and coincidences, and the mathematical beauties in which solids seem inclined to clothe themselves.

The father of true physical philosophy has shown that the squares of the periods of the planets are as the cubes of their mean distances from the sun; and wishing to be justified or corroborated in my determination of the quadrature, by that great physical law in regard to force and motion, I will here make a reference of the consequences, of my determination, to the two great Keplerean laws; and, in such reference, shall (for the present purposes) consider orbits of different dimensions as being circles of different dimensions; premising that while the circle whose diameter is unity is the prime circle, the orbit whose $r a$ dius is unity is properly the prime orbit; and this for reasons that will readily be recognized. And I will here remark, that in general we have as little to do with the area of an orbit in the investigation of the laws of force and motion, as we have with the areas of polygons in the investigation of the measures of the circle.

Unity is the standard measure for anything susceptible of numerical admeasurement, or of ratio and proportion; hence, when the radius of an orbit, or the mean distance of the planet is 1 , the period is 1 ; else the square of the period could not be as the cube of the distance; so when radius is 1 , the force of gravity must be called 1, - for such is the only condition upon which we can proportion the rate of gravity to different distances from the same attracting power. So also, at the distance 1 , the mean rate of motion is 1 , as also the rate of convergency of the orbit, and especially the time of the period.

I will here assume the fact, that if the diameter of the inscribed circle of any given polygon be $=$ to the square of the circumference of a like major polygon, the circumference of such polygon will be the third power of the circumference of such major 
polygon; and the same will hold good in respect to the circle, or orbit.

Hence, when the diameter of an orbit is $=$ the square of the circumference of the circle whose diameter is 1 , the circumference of such orbit will be 32 , or one fourth of the circumference will be 8 .

Or thus: when the radius of an orbit is four times the square of the circumference of the prime circle, the square of the circumference of such orbit will be the third power of radius (the mean distance) of such orbit; and such necessarily occurs when the circumference of the orbit is 256 , in which case, the period will be $=$ the circumference.

Or the version may be thus:-if the circumference of an orbit be the product of the third power of the circumference of the prime circle, by 8 , the period will be $=$ the circumferencefor when the diameter of an orbit is 8 , the period is $=$ the diameter, by any system of the circle.

So when the diameter of an orbit is 8 , the circumference is twice the square of the circumference of the square (or $p 4$ ) of equal area with that of the prime circle.

The third power of the circumference of an orbit is 32 times the third power of the diameter, and 256 times the third power of the radius; so when the circumference is 256 , the square of the circumference is the cube of radius.

So when the circumference is 64 , the period will be 32 , and when the circumference is 16 , the period will be 4 , and so on. So when the circumference is 4 , the period is one eighth of the circumference; viz., the period will be .5 .

Thus, when the radius of an orbit is the third root of any given point, the period of the planet is the second, or square root of the same point. And hence we may conclude that this great law is founded in unity of purpose.

Suppose then a planet to revolve in a centric orbit, namely with the sun in the centre, and of course having a uniform motion: Then if the force of gravity be inversely as the distance, (as I allege it to be,) the rate of motion will be the square root of the force applied; but if the force of gravity varies inversely as the square of the distance, as taught by Sir Isaac Newton, then the rate of motion will be the fourth root of the force applied; as may readily be seen by almost any one who will bestow a little consideration on the subject. The circumferences of circles are as their diameters, or as their radii ;- hence, if Kepler, could have supposed that his great law, as by him declared, could have been mistaken or perverted, he might have declared anew, that the third power of one eighth of the circumference 
is half the square of the period; or that the square of twice the period is the third power of one fourth of the circumference, \&c., thus comparing time with circumference, in lieu of diameter; and the law thus declared would have been equally true and equally well received. For then, as now, when the circumference is 4 , the period will be .5 , or equal to oneeighth of the circumference ; and still, when the diameter is 8 , the period will be 8 , and when the period is 4 , it will be $=$ onefourth of the circumference, or the square root of the circumference, \&c.

When the period is .5 , or the square root of .25 , the radius is necessarily the cube root of .25 , or the square of 2 , and the di-

- ameter must be the reciprocal of $\underset{2}{2}$, by any system; else the Keplerean system must fail.

This statement, however, will not quadrate with the popular measures of the circle; and it will be seen and acknowledged, that in applying the popular measures of the circle to the laws of planetary force and motion, it requires a great deal of calculi to chink up with. According to this, the third power of onefourth of the circumference of an orbit when the diameter is 1 , is equal to the period of a planet in an orbit whose circumference is $4 ;$ - but not so says the popular method.

By any system, the rate of motion, and the period of a planet have a determinate ratio to the diameter of the orbit; but according to the popular method, they have no determinate ratio to the circumferential measure of the orbit; that is, according to the popular notions, neither the motion nor time of the period, have a determinate ratio to that of the line or path in which the planet actually moves or revolves.

By any system, when the circumference is 4 , or when the area is = the diameter of the orbit, the period is the third power of the diameter of an orbit whose area is .5, - hence, the period of any planet of a system is = the third power of the diameter of another orbit of the system whose area is =, one-eighth of the circumference of the given orbit. Thus, if the circumference of an orbit be 256 , the period is = the third power of the diameter of an orbit wh se area is 32 .

This application may also be made to orbits in connection with polygons, namely, the reciprocal of $M$ of any given polygon is the diameter of an orbit in which the period is the third power or cube of the diameter of the circumscribed circle of a like polygon whose area is .5 .

If the limits assigned to this work permitted, it would by no means be an unpleasant task to contemplate the relationship of the family of curves; and wherein the parabola (that curve of all others except that of the circle, the most useful and impor- 
tant to be understood,) conspires with the circle in the admeasurement of solids, \&c., and serves to corroborate my determination of the quadrature.

It is entirely beyond my sagacity to determine how the ellipse (or elongated circle) has been elaborated into notice and consequence far beyond its merits, otherwise than by way of mathematical or geometrical discipline to the mind; and I am equally uninformed why the science termed the science of conic sections, (from which the modest circle is generally excluded,) has been regarded paramount to any other department ; - of which science it is 'said, by those in high places, that " it forms one of the most important parts of mathematics, being distinguished for elegance, and demonstrating, with surprising simplicity and beauty, and in the most harmonious connection, the different laws, according to which the Creator has made worlds to revolve," \&c. - " that few branches of mathematics delight a youthful mind so much as conic sections; that the emotions which the pupil manifests when they unfold to him the great laws of the universe, might be called natural piety; and that in teaching this science to young people, the descriptive method (by diagrams) ought always to be connected with the analytic method," \&c.

I am aware that the ellipse, the parabola, and the hyperbola, may be presented in a train of diagrams which in their appearance are very imposing; and that they may'serve in connection with the importance which is generally supposed to be attached to their explication, to induce a species of faith or belief, which some would call natural piety. But I have yet to learn that this science discloses the laws according to which the Creator has made worlds to revolve ; or that, - as has also been admiringly expressed, - in comparison with conic sections, in view of the grandeur and beauty which they unfold to the mind in the mathematical world, all that has preceded this part is but the alphabet of the science of mathematics, \&c.

It is true that the strong grounds thus far, of the Newtonian philosophy, is in the belief that he disclosed, by their aid, the law according to which the Creator has made worlds to revolve; and so tenacious have been the sustainers of Newton's fame, that the cry of robbery was raised when Bernoulli made the allegation without giving Newton the credit, that "we now know that the heavenly bodies perform their revolutions by conic sections ;" thereby inducing the world to attach vast importance to what may, at some future day, be shown to be wholly delusive.

It is somewhat curious to contemplate the means and the ingenuity which have been practised, to induce the natural piety 
conceived to flow from a contemplation of conic sections; and it is certain that those articles of faith have been fortified by great care and caution, and by a continual inculcation; so much so, that not a word must be suggested, in our works on astronomy, that it would be possible for a planet to revolve in a circle, whether centric or eccentric; nor that the ellipse can be cut from any other solid than the cone. Hence, the natural minds of thousands have been so continually plied with the elliptical orbit, and with the magic qualities of the cone and of conic sections, that at length they have yielded, or, at least, rendered passive obedience. And it would not be wonderful if something strange should come over the mind, when it is brought to assent to the Newtonian hypothesis, that the Creator has caused worlds to revolve in ellipses, however eccentric.

Sir Isaac Newton settled many knotty questons by his favorite expression, of Neque novit natura limitem, which, perhaps, may have various renderings, for it will as well apply to error as to truth; for when we have set out on the road to error, we can find no bounds or limits to our career; and such is, doubtless, the case in respect to the hypothesis of elliptical orbits, - as I shall attempt to prove, - an error which commenced with Kepler, while making calculations upon Tycho's observations; an error which, although not depending upon any physical necessity or law, was seized upon by Newton; and he has given it such importance, as nearly to obliterate the two great laws of Kepler, and especially that which declares that the squares of the periods are as the cubes of the mean distances, - the modern version of which is, that " the squares of the periods of the planets are as the cubes of their semi major axes."

If such then be the law, - namely, that a planet may revolve in an orbit, however eccentric, even to extreme eccentricity, and that the square of the period is the cube of the semi major axis, - it is manifest, that during the period of such planet, a vastly greater amount of the force of attraction will be applied to it, than to a planet revolving in a circle, (whether centrio or eccentric,) whose diameter is = to the major axis of the ellipse ; while the planet revolving in the ellipse would pass over less than two-thirds of the space of that revolving in the circle, in the same time or period, and a far less proportion of area. Hence, the popular notion makes the time of the period depend wholly upon the major axis of the orbit, without regard to amount of space passed over, or the amount of force expended during the period. And such have been the doctrines and teachings which have induced what is termed natural piety, in those who have been induced to conceive that such things could be. 
If it shall be found, then, that a planet cannot revolve in an orbit of extreme ellipticity, if we retrace the road which led to such error, we shall find that a planet cannot revolve in an elliptical orbit, however small the ellipticity.

I will, however, in conclusion of this reference, suggest a dictum or two, based upon the operation of the Keplerean law, namely, that the square of the period is the cube of the distance, and that the radius vector passes over equal areas in equal times; .consequently, that the motion of the planet, at any given point of the orbit, varies inversely as the distance varies, - which laws, in fact, are one and the same, operating in unity of purpose, although the same has generally been resolved into two separate or distinct laws. This law, then, taken in connection, presupposes its operation in an eccentric orbit, or one in which the central force is not situated in the centre of the orbit.

This subject, however, has been dark, mysterious and inexplicable, in consequence of the Newtonian error, in assigning the law; and notwithstanding it had become much the fashion implicitly to avow that to be true which it was said Sir Isaac Newton had found, nevertheless, for many years after the promulgation of the Newtonian law of gravity, the truth had not become so far entangled in the meshes of error, as to place it wholly beyond the power of redemption, until Clairaut effectually accomplished it, in his supposed accounting for the motion of the moon's apogee, upon the principle of the Newtonian law of gravity; for, previous to that time, it had not been deemed high treason for men in high places to speak disparagingly of the law.

But still the square of the period is the cube of the mean distance, and still the motion varies inversely as the distance varies, and consequently the line of the radius vector passes over $=$ areas in equal times ; and, consequently, the simplicity in the laws of force and motion, by which the heavenly bodies revolve, should not have been rendered thus complicated and inexplicable. For the above phenomena once being established, directly and conclusively determine the law of gravity, by which the phenomena are caused or produced ; that is, the law is directly deducible from its effects; and why it should not have been done, is somewhat unaccountable.

I allege, then, that the intensity of gravity is inversely as the distance, and, consequently, that the mean motion of a planet is the square root of the mean force; (not that the mean motion is the fourth root of the mean force, as Sir Isaac Newton would have it.)

Then, as the motion of a planet in an eccentric orbit varies inversely as the distance varies, hence the motion, at any given 
point of the orbit, will be the square of what the motion of a planet would be, in a corresponding, point of a like orbit, whose mean distance is the square root of the mean distance of the given orbit; and this for the reason, that the mean motion of a planet is inversely as the square root of the mean distance; else the squares of the periods of a system of planets cannot be as the cubes of their mean distances from the gravitating power.

Hence, if the mean distance be 4 , and the perihelion distance 1 , the actual motion, at any given point of the orbit, is the square of what the actual motion of a planet, revolving in a centric orbit, would be, at just half the distance of such given point. Thus, if the mean distance be 4 , the mean motion will be .5 ; or the square of the motion of a planet, revolving around the same central force in a centric orbit, at the distance 2 , or at half the mean distance of the given orbit. And if the perihelion distance of such orbit be 1 , the motion at the perihelion would be 2 , or the square of the motion of a planet, revolving in a centric orbit about the same central force, at the distance .5 , or half the distance of the given planet at perihelion, and the like would be the case in any point of the orbit.

And such will be found to be some of the necessary and inevitable results of the law of gravity, and they are in perfect accordance with the great phenomena observed by Kepler, namely, that the squares of the periods are as the cubes of the distances; that the motion varies inversely as the distance varies; and, consequently, that the radius vector passes over equal areas in equal times, and that none of these phenomena can occur, except the force of gravity be inversely as the distance.

\section{SECTION SIXTH.}

Infallibility is certainly a rare quality to acquire during the whole period of one's life, and but few may expect to be charged or credited with it; and I certainly have no desire that the world should enter upon their books any such charges or credits to me; and shoúld $I$ be charged with an attempt to mar the record of the world in respect to the infallibility of Sir Isaac Newton and some others, for whom the votaries of science have long claimed the entire admiration of the world, I have only to remark that such admiration has savored quite too much of idolatry to subserve the best interests of science; and I have never yet believed that the only proper way to extend or perfect science in the eyes of a wondering or admiring world, is, by inducing a belief in the infallibility of some one of its votaries who may have essayed to 
lay its broad foundations. And having honestly conceived that certain principles promulgated by $\mathrm{Sir}$ Isaac Newton as fundamental, (and so received and taught by the world,) are wholly erroneous, - and that long before this the errors would have been detected and repudiated, but for the admiration of the supposed infallibility of Sir Isaac Newton, so diligently and successfully inculcated, - I have not hesitated to attempt a reformation, by pointing out such defects and want of comeliness in the object of adoration, as I supposed could not well be overlooked, even though the charge of jealousy of the fame of Sir Isaac Newton should be as industriously sounded abroad against myself as it has been against Liebnitz, Hook, Bernoulli, and others of Newton's cotemporaries, who were to be properly disposed of, that Newton might be regarded by the world as "the creator of natural philosophy." For such has been the constant practice of the world; nor have our ears been in anywise relieved from the trump of fame, notwithstanding the modern disclosures in respect to his treatment of Flamstead, and the indignation consequent thereon, to allay which has even required the able pen of Mr. Whewell.

Thus, in most English treatises upon science, we are constantly reminded of the quarrel between Liebnitz and Newton, in respect to priority of discovery of fluxions, or the calculus, considered so important in the involution and evolution of curves, \&c., which, after having been introduced by the celebrated $\mathrm{Mr}$. Huygens in the legitimate application of the cycloid to pendulums of clocks, has certainly been carried to great lengths in speculative philosophy, even to those doubts and difficulties which are acknowledged to be almost inexplicable, and which, perhaps, of all the branches of science, best serve to give the history of the war between Newton and Liebnitz, which is often set forth in such descriptions as might enable a Fuseli to depict those giants, each having hold of a horn of the dilemma, and contending manfully for the milk. 'But I protest, that it is both the privilege and the duty of every free born son of earth, to be jealous of the fame of any one, if such fame shall tend to check or stop the upward progress of man, either moral or scientific, to which he seems destined by a proper development of his capacities.

It is said, however, that "the evolution and involution of curved lines have great claims on our attention;" and that "this doctrine is peculiarly valuable to the speculator in the higher mechanics." And in fact, we find that the thread of involution and evolution has been industriously interwoven into the mechanics of the heavens, by ingenious processes, which are quite 
surpassing common comprehension, and therefore destined to remain, except to the initiated, as one of the mysteries of science.

Nevertheless, one great object of this branch of science is, to ascertain the flexure or variation of curvature in any given point of any curve other than that of the circle, (as that of the parabola, for instance,) when compared with the uniform curvature of the circle, whose magnitude is so adjusted to the occasion as to give it the name of the equi-curve circle; in which case the curvature of such circle is conceived, or supposed, to be equal to the curvature of the parabola in the given point. And it is said, " had Appollonius but noticed a single fact, he would have discovered the whole theory of evolution, and its importance in speculative geometry.

Thus, in the case of involution and evolution, as in the case of conic sections, the circle is considered to be the standard measure, to which other curves must be referred in our investigations; and hence, if there be error in assigning the ratio of the diameter of the circle to the circumference, such error is necessarily visited upon every other curve of which the circle is necessarily the standard.

The theory, then, of involution and evolution of curve lines, as delivered by Mr. Huygins, (and which is said to have been highly prized by Newton, who applied it to trochoids and epicycles of all kinds, but which is said to have been finally eclipsed. by Newton's fluxionary geometry,) may properly be said to have been the progenitor of fluxions, and is said to be peculiarly adapted to fluxionary equations, by which the fancy may conceive infinities and infinitesimals to such an extent, that the greatest of a never-ending series of one kind of magnitudes, shall be infinitely less than the smallest of another successive series. This extension of nothingness has, however, given occasion for such eminent metaphysicians as Newton, Liebnitz, Bernoulli, \&c., to accuse each other of giving explanations of orders of curvature that can have no existence.

Nevertheless, whoever shall carefully examine the subject will perhaps perceive but little actual difference in the extension of their respective imaginations: All agree that there is a difference between the simple contact of two curves, and the point in which the circle shall osculate a curve, or the point of osculation; and hence, that there can be a closer union between the curve of the circle and another curve, than that produced by simple contact.

Liebnitz speaks of different degrees or orders of osculation, each of which is infinitely closer than the other; and it is contended, in honor of Newton, that he had done this before, \&c.; and that Liebnitz's allegation, that an osculation produced in the evolution of a curve is equivalent to four intersections, is alto- 
gether erroneous, for it is contended " that the circle indicated by the coalescence of three intersections is properly named the equicurve circle; that since we measure all curvatures by that of the circle, such circle is properly the circle of curvature, and its radius is the radius of curvature; for the flexure of the circle being the same in every part, it becomes the proper index for all curves," \&c. Hence it is said that "where two intersections coalesce, there is a common tangent; and when three intersections coalesce, there is an equal curvature, - for then no other circle can pass between this circle and the curve."

But it is again and again alleged "that there may be indefinite degrees of this coalescence of closeness of contact between a curve and the circle; and that this gradation of more and more intimate contact, or approximation to coalescence, may be continued without end : Neque novit natura limatem." And such has been the extent to which this perplexed metaphysical reasoning has been carried, that evolutrixes have even been conceived or imagined, which coincide with straight lines, and others of infinitely greater rectitude which still are curves.

Now, however well such a course of reasoning may serve as an exercise for the metaphysician or speculative geometer, it may nevertheless appear to most people of plain common sense, illy adapted to any beneficial purpose or practical use, and may remind one of the eulogy pronounced upon Newton's defence of his theory of optics, or analysis of light, against the attacks of Hook, Huygins and others, in which it is said his minuteness of detail was so inconceivably beyond the ken of mortals, as that very few were able to comprehend his reasoning.

And it may be doubted whether those who contend for a never-ending approximation to a closer and closer contact or coalescence, than that of a simple contact, have not left the substance to grasp at the shadow, and thereby extended their conceptions beyond the reality of things; for if a point be but position without length or breadth, and if a line be but extension without breadth, it may be hard to conceive how two points or lines can approximate nearer to each other than simple contact.

But such fantastic reasoning seems rather calculated to operate as conviction to the world, than as legitimate proof in the premises; for in view of the doubts and perplexities of those who have essayed to give the evidence; (and their mutual jealousies forbade their settling the matter by convention,) it is doubtful whether a great portion of mankind will undertake a reinvestigation of the subject; whether they will not rather prefer to admit such reasoning to be beyond their comprehension. And hence the world at large (with few exceptions) assent that such may be an exposition of those laws, "according to which 
the Creator has caused worlds to revolve," in accordance with the Newtonian theory and law of gravity, namely, by the sole and constant operation of disturbing forces; and hence it is said, and generally believed, "that all these curves and angles of contact, may be, and certainly are, every day described by bodies moving in free space, and acted on by accelerating forces directed to different bodies."

Those conclusions, arising from attempts to fathom infinity by progressive steps, and in which we are as liable to pass the goal as to fall short of it, have doubtless led to as much of error as they have of curious and unprofitable speculation. They have entangled the theory and law of gravity in those meshes from which the world has been unable to extricate them; and although those mysteries seemed neither designed nor calculated to develope or disclose the true theory or law of gravity, they have nevertheless been supposed (some how or other) to establish them according to Newton. Perhaps the careless or unskilled observer would never discover any reason why a piece of complicated machinery might not possess the power of producing perpetual motion, if it would but go; but Newton has wholly the advantage in the parable, for, thank heaven, the system of worlds continues to move, however rickety it may have become in consequence of having been transferred from the laws of Nature to those of Newton.

Such, however, has been the struggle for the mastery in metaphysical reasoning, with a view to establish the physical laws of the universe to the satisfaction of the world, as to have enabled certain philosophers, high in estimation as such, to play off before our blunted visions, their corpuscules or physical atoms, whereby they would attempt to explain the centre of gravity between them, the curves which they would describe while revolving round the centre of gravity, and around each other, \&c.; and thence, for our edification, transfer those imagined laws to the solar system, or universe; and so ready have we been to be satisfied with the greatest, or most approved player, that opposition has given greater importance and impulse to the Newtonian philosophy, than even passive assent or non-resistance. And it may well be apprehended that it was more from some knowledge of the fact, than from inspiration, that Milton was enabled to furnish his Raphael with so appropriate an answer to Adam's inquiry in respect to the movement of the heavenly bodies. But a fig for the inspiration of a poet who must see events fulfilled before he can sing them.

Notwithstanding, it is properly alleged, and generally assented to, that "curvature is a change of direction only;" neverthe- 
less, the subject has been much perplexed through undue or improper considerations; and hence we find so many cases put, the declared object of which is to assist our conceptions in respect to the popular doctrines on what is termed a delicate subject.

Now the same visionary mode of reasoning, (for it cannot be philosophical,) which conceives a closer and closer contact in the point of osculation as the magnitude of the circle is decreased, leads to the idea or conception of curvilinear angles which are incommensurable and incomparable; thus an osculation is said to denote an assignable angle; and hence will arise that unmeaning expression of an angular motion of a heavenly body; and although the circle is properly named the measure of all angles, nevertheless, neither the periphery of the circle, nor of any curve line, is supplied with angles, nor can they properly be said to form angles with their tangents, or greater or less angles with their tangents.

As is properly said, curvature is a change of direction alone. This change of direction may be either greater or less; and hence may be said to denote, indicate, or determine, what may be called the curvature or convergency; and if referred to unity, it will indicate what may properly be called the rate of curvature; or, as I have chosen to call it, while treating of the laws of force and motion of the heavenly bodies, the rate of convergency, which rate of convergency I proportion to that of the rate of force or gravity by which it is produced, very differently from Sir Isaac Newton.

Now it would seem if what may be called curvature, or convergency, is an element for consideration, in an investigation of the laws of planetary force and motion, that the same standard should be assumed by which its rate may be measured and cornpared; and such standard I have placed in unity; nevertheless, we find the most approved Newtonian philosophers and commentators, - while treating of what is esteemed so very important a subject as that of the Newtonian department of the involution and evolution of curves, - treating the subject after this manner :

"We speak of curvature that is greater or less; and every person has a general knowledge or conception of the difference, and will say, that an ellipse is more curved at the extremities of the transverse axis, than any where else. But before we can institute a comparison between them with precision, \&c., we must agree about a measure of curvature, and say what it is we mean by a double or a triple curvature.

"Now there' are two ways in which we may consider curva- 
ture as a want of rectitude; we may call that a double curvature which, in a given space, carries us twice as far from a straight line; or we may call that a double curvature by which we deviate twice as much from the same direction. Both of these measures have been adopted; and if we would rigidly adhere to them, there would be no room for complaint; but mathematicians have not been steady in this respect, and by mixing and confounding these measures have frequently puzzled their readers. All agree, however, in their first and simple measures of curvature, and say, that the curvature of an arch of a circle is as the arch directly, and as the radius inversely. This is plainly measuring curvature by the deflection from the first direction. In an arch an inch long, there is twice as much deflection from the first direction as when the radius of the circle is of half the length. If the radius be about $57 \frac{1}{4}$ inches, an arch one inch in length will produce a final direction one degree different from the first. If the radius be $114 \frac{1}{2}$ inches, the deviation will be but half a degree. The linear direction from the straight path will also be one half. In the case of circles, therefore, both measures will agree; but in by far the greatest number of cases they may differ exceedingly, and the change of direction' may be greatest when the linear deviation is least.

"Flexure, or change of direction, is, in general, the most sensible and the most important character of curvature, and is understood to be its criterion in all cases. But our processes for discovering its quantity are generally by first discovering the linear deviation; and in many cases, particularly in our philosophical inquiries, this linear deviation is our principal object. Hence it has happened that the mathematician has frequently stopped short at this result, and has adapted his theorem chiefly to this determination.

"These differences of object have caused great confusion in the methods of considering curvature, and led to many disputes about its nature, and about the angle of contact; to which disputes there will be no end, till mathematicians have agreed in their manner of expressing the measures of curvature. At present we abide by the measure already given, and we mean to express by curvature or flexion the change of direction."

This, however, does not indicate any great unity of purpose in the consideration of curvature or convergency; nor could the mathematicians well settle the question by convention, so as to avoid discrepancies in the application.

Now the same author above quoted says, that "the doctrine of curves by involution and evolution is peculiarly valuable to the speculator in the higher mechanics. The intensily of a deflecting 
force is estimated by the curvature which it induces on any rectilineal motion; and the variations of this intensity, which is the eharacteristic of the force, or what we call its nature, is inferred from the variations of this curvature."

And the inference which they have drawn is properly referred to an examination of the Newtonian method of investigating the laws of planetary force and motion, in which some proper estimation is attempted to be given in respect to the curvature or convergency produced in a given time at a given distance, \&c.

That portion of the science, however, of the involution and evolution of curves, which, from its supposed transcendentalism, is so strenuously claimed by Newton's friends and admirers, to be wholly of Newtonian origin, seems to have been left somewhat perplexed, both as to enunciation, demonstration, and application; for there may yet be some doubt in respect to the magnitude of the equi-curve circle, or rather in regard to the point in which it osculates the curve; for Newton himself perceived the closer and closer contact as epicircles are drawn of less and less magnitude, ad infinitum; and inasmuch as a mere point without length or breadth is incapable of being curved,-it may yet be the case that the only method in which the equi-curve circle can be settled and established, is by convention; and more especially, inasmuch as the osculating circle (as named by Liebnitz) forms an actual or assignable angle with the curve supposed to be measured. By such means, then, they obtain their fluent for evolving the curve, when it is acknowledged, that after exhausting every device, no fluent could be found for evolving the circle. This portion of the science, however, is not without its use to the speculative geometer in the higher mechanics, as it enables the expert symbolical analyst to equate the motions of the heavenly bodies to their crinkled orbits, even though they assume the force of gravity to be inversely as the square of the distance.

Now in respect to the simple cycloid, if we conceive the prime cylinder (namely, that the diameter of whose inscribed sphere is unity) to be rolled upon a horizontal plane, so as to perform or describe just one entire revolution, the area of the arch formed by that line of the cylinder which rests upon the plane at the time the revolution commences, as also when it ends, is 4 , or $=$ the lateral surface of the circumscribed cube of such cylinder; while the base of such arch formed on the plane by such revolution, is = the lateral surface of such cylinder; or is = the entire surface of the inscribed sphere of such cylinder. It is also $=$ the greatest ellipse or elongated circle that can be formed in the rectified surface of such cycloidal arch; and the bulk contained between such cyloidal arch and its base, will be three times the 
bulk of the generating cylinder. The area of the greatest parabola that can be drawn within such arch, is = four times the bulk of the inscribed sphere of the generating cylinder; or is = 8-9ths of the area of one end of such cycloidal arch, or is to the area of one end of such cycloidal arch, as two thirds of 1 is to three fourths of 1. But the subject is easy of contemplation.

It will be seen from the gathered hints of Sir Richard Phillips, that Sir Isaac and Sir. Richard differ very widely in their estimation of the fall of the moon from a tangent in one minute of time, from which Sir Isaac deduced his proof of the law of gravity; Sir Isaac making the space about 16 feet, while Sir Richard makes it about 130,000 feet. But admitting that Sir Isaac has been mistaken, even to the extent alleged, such mistake was by no means the cause of the tremendous error committed by him in respect to the law of gravity.

Perhaps the world will yet come to the conclusion that what is termed Newton's first law, or the axiom which he assumed for the government of the world's philosophy, is wholly gratuitous and uncalled for, being merely an assumption without having any known foundation in the universe; and that his second law or axiom should at least have stated what proportion the motive force impressed, bears to the change of motion of a body. Nevertheless, he ought to have been convinced, from the reasoning which naturally flows from his first two corollaries, that the motion is the square root of the motive force applied; and consequently, that the force of gravity is inversely as the distance, and not as the square of the distance. But, of all the discoveries for the bewildering and misleading of philosophy, perhaps nothing could have been contrived that could so perfectly mystify and darken counsel; that could so effectually direct the mind into an infinite and inexplicable mysteriousness, where it was supposed ultimate truths alone existed, as do those lemmas upon which his mathematical philosophy chiefly rests; and from which those refined mathematics have emanated, which have enabled La Place and others to subdue the phenomena of the heavens to the Newtonian theory and law of gravity.

Thus the mystical terms ultimate ratios and ratios of equality, have induced the belief that Sir Isaac Newton found that the ultimate ratios of the sine, chord, and tangent of arcs infinitely diminished, are ratios of equality; and hence that in our reasoning, we may use the one for the other; and having once commenced such a train of reasoning, we must come to the conclusion that the ultimate form of evanescent triangles formed by the arc, chord, and tangent, is that of similitude, and their ultimate ratio is that of equality, and that each may be used for the 
other. And hence has been deduced that futile and impracticable method of finding the length of the arc of a circle in terms of the sine, versed sine, tangent or secant, by means of fluxions; that is, in geometrizing upon a purely geometric point, namely, upon nothing, or mere position; we may conceive it to be in what shape we please; that its ratios are ratios of equality, and that they are all ultimate ratios; and hence, that the evanescent triangle may be in form of the circle, and vice versa. But such has been the philosophy (if we may so speak) which has led the world to strange conclusions.

Now it is said that Sir Isaac Newton showed that the space which a body describes by any finite force, whether determinate, or continually varied, are to each other, in the very beginning of the motion, in the duplicate ratio of the forces. But this is directly deduced from the simple law of falling bodies, which had long before been determined by Galileo. Nevertheless, the fact, however disclosed, should have taught Newton, or any other person, that the force of gravity is not inversely as the square of the distance; but that it is inversely as the distance. 


\section{H A P T E R II.}

On the Law of Gravity, and the Popular Deduction and Promulgation of the Supposed Law.

\section{SECTION FIRST.}

Gravity may properly be defined to be (at least for the purposes of this article) that physical power which pervades the solar system, (and perhaps, judging by analogy, the universe,) displayed or manifested to our senses in and through the medium of tangible matter, by which the phenomenon is constantly presented to our perception, of aggregate bodies of matter attracting each other, at least under certain circumstances; and hence we observe bodies falling from a state of rest towards the centre of the earth; and hence the centripetal force which retains the planets in their orbits around the sun, and the satellites in their orbits around their primaries. To present and more readily compare the popular notions of gravity with suggestions or hypotheses which I may attempt to intrude in place of them, I will suggest the consideration of the subject under two heads, namely: the theory of gravity, and the law of gravity. The theory of gravity will mainly be referred to the momentous question, - whether tangible or aggregate matter, of itself, is endued with or possessed of innate or inherent gravity, or an inherent power to attract and be attracted, just in proportion to the quantity of aggregate matter, wherever situated, in the solar system, (for I will not attempt to investigate beyond that,) which theory may be termed the theory of inherent gravity; - or, whether the power incident to a given quantity of matter to attract, or the susceptibility to be attracted, must depend upon some numerical law in respect to its distance from the sun, or great centre of gravity of the solar system, - which may be termed incidental or incited gravity.

The law of gravity, in this division of the subject, will simply consist in the rate, intensity or efficacy which the force of gravity 
exerts, between two given quantities of matter, in proportion to the distance between them at which it shall operate; and hence, to determine the numerical law of gravity, is simply to determine the rate or intensity in proportion to distance. And in the investigation of such ratio and proportion, unity or 1 occupies a very conspicuous numerical point or position. If the theory of inherent gravity shall prevail, it will be necessary to consider the theory and law of gravity under distinct heads ; and consequently, that the theory may be brought to harmonize with the law in some of the principal phenomena produced, some sufficient cause must necessarily be assumed. Hence we find that Sir Isaac Newton assumed that planets at a greater distance from the sun were less dense than those which were nearer; and consequently that their powers of gravity were as their respective bulks multiplied into their rates of density, or as their quantity of matter in proportion to their bulk; but as the supposed densities of those planets having no satellites, could not be as readily determined and proportioned as those which had satellites, resort was had to their supposed disturbing forces operating on other planets; and hence their supposed densities were variously assumed, according to the supposed evidence obtained.

So nearly, however, have such men as Euler, LaGrange and others found their supposed proportional densities to agree with their proportional distances from the sun, (namely, in the inverse ratio to that of distance,) that they, and especially LaGrange, founded astronomical tables upon this hypothesis; and long after, he remarked that he saw no occasion to vary from a strict adherence to such hypothesis. - To be sure, Herschel, the astronomer, assumed the density of the planet Herschel to be greater than that of Saturn; but modern discoverers have determined and shown that Herschel was mistaken in respect to the motion of Herschel's satellites ; and consequently in respect to the supposed density of the planet; and in regard to the supposed density being proportioned to the distance, as with other planets; and hence upon the theory of inherent gravity, the proportional densities of the planets are assumed to be inversely as their respective distances from the sun, for the reason that the power of gravity which they appear to exert is in proportion to their respective distances from the sun, or the centre of gravity of the solar system. But may it not well be doubted whether theoretical or hypothetical philosophy was well applied in attempting to deduce the cause from the effect, - from the facts and the phenomena observed, - when a want of density in proportion to the bulk was assumed as the cause of the want of gravity in proportion to the bulk? This depends, however, upon the premises 
from which we draw our inferences; as such a deduction of the cause is at best but an inference, and should be drawn from the best and greatest amount of evidence that may be brought into the case, and should not be left to rest for its truth or final support, upon some abstract hypothesis previously assumed, - as for instance, the innate or inherent gravity of matter. If the attractive power of matter be inherent in it, and that too in proportion to the quantity, wherever situated in the solar system, then, to be sure, the popular inference, - that the proportional densities of the respective planets are as their bulks multiplied into the inverse of their respective distances from the sun, - is well founded, because their proportional attractive powers are such; but perhaps if the naked or abstract hypothesis of the inherent gravity of matter had not been brought in as a make-weight, philosophers would not have seriously thought of making the popular deduction, or of drawing the popular inference in respect to the different densities of the planets, especially from any observed facts or phenomena, but would have stricken from the declaration such allegations as were not proved, and not founded upon the evidence; that is, they would have travelled upon facts, so far as they were plainly to be discovered, and let the inferences or deductions follow in their proper places; and instead of alleging that the proportional densities of the planets are respectively as the product of the bulk $X$ the inverse of the distance, they would have said that the proportional power of gravity exercised in, by, or through the respective planets, is as the product of the bulk $X$ by the inverse of the distance, (for here is a fact found,) and would have left the cause (if not yet discovered through the medium of facts or phenomena) to be assigned whenever warranted, instead of assuming a naked, unsustained allegation, as the cause of the phenomena observed.

Let the inquiring mind, then, conceive, in accordance with the popular notion or hypothesis, that the planets become less and less dense in proportion to their distance from the sun, and it will inquire for the cause of this very curious phenomenon, this phenomenon apparently fraught with some unity of purpose, - some indicative of some necessary law, by which the solar system (if not the universe) is governed; and it would be slow to believe that all this order and harmony is merely fortuitous, or the result of chance; nor would it be very likely, on serious reflection, to base or assign the cause in a naked and wholly unsupported assumption; and especially if such assumption were in direct derogation of all the physical laws and operations of the universe, so far as we are able to enter into their examination and their operations, by means of such puny attempts and 
trials as we are enabled to make. Neither would the inquiring mind, - with the view to cut short its inquiries, and dispose instanter of a question or hypothesis in reference to which the universe is to be squared or quadrated, and upon which the whole superstructure of astronomy is to be reared, - be likely to adopt as the cause of the most astonishing order and harmony in the solar system, that naked hypothesis which makes the whole physical power of the universe, - a power to be constantly exercised at immense distances, - to be inherent in inert or dead matter. If it be possible to have a clear conception of such a matter, it is but conceiving a state of things existing per se, or, as some have imagined the case to have been, when the earth was without form and void. And will any one pretend that such hypothesis is any improvement upon the heathen mythology, which places fate beyond the power of the gods to control it? I say, the inquiring mind will hesitate before it adopts as the basis of the whole fabric of astronomy and philosophy, a naked allegation or hypothesis, which, if true, so fully dispenses with any law of order by which we hope and believe the universe is upheld, controlled and constantly governed, and converts the whole into a system (if such it may be called) of disturbing forces, - that is, a system which constantly disturbs itself into an eternal harmony. Unless, however, it had been believed, (as many good and eminent men have believed implicitly, even to the endorsement of the idea from the pulpit,) that the projectile hypothesis of Sir Isaac Newton, - namely, that the planets were originally projected by the Supreme Being in tangents to some given point of their respective orbits, - was a sufficient development of a Deity, and of his doings in upholding and governing the solar system, and, by analogy, the universe, such hypothesis could not have prevailed. But I dare not so rashly presume or assume, but if I shall be able satisfactorily to determine something in respect to a numerical development of the great physical law by which the solar system is upheld, sustained and governed, I shall be satisfied to dispense with mere naked and presumptuous allegations, which, if issuing from a man proudly sustained, as was Sir Isaac Newton, might plunge the world into errors from which centuries could not extricate it.

The true theory of gravity, then, is yet open to investigation; and whether the force of gravity be a power inherent in matter, or whether gravity or attraction be an omnipresent law, operating on matter in proportion to distance from a centre of gravity, - is a question which ought to be determined upon the best evidence the nature of the case will afford. If, however, it 
should be discovered that the attractive power of a body of aggregate matter in the solar system is only induced, and that, too, in proportion to its inverse distance from the sun, or centre of gravity of a system, the theory of gravity will become so far involved in a consideration of the law of gravity, as to require no distinct or separate consideration from that of the law.

In the consideration or investigation of this subject, it will be my object to bestow most attention upon the law of gravity; namely, its rate or intensity as proportioned to the distance from what may be properly termed the attracting body, or that which induces, or from which appears to emanate the centripetal force which retains a revolving body in its orbit; treating, however, of the Newtonian theory of gravity in an incidental manner, whenever it may seem necessary by way of explication. It may here be remarked that the Newtonian method of investigating the law of gravity by a consideration of the law of falling bodies towards the earth, has been preserved with much care by those authors who have quoted or given us the method, and who have commented on it. See Maclaurin's account of the Newtonian philosophy, in four books, Rees' Cyclopædia, Vince's Universal System of Physical Astronomy, \&c.; the investigation seems to have been extremely simple in its character, and, from that cause alone, would not seem calculated either to lead mankind into error, or to keep them there, if peradventure they were thus led; for the investigation certainly contains nothing more intricate than the consideration of a numerical formula, designed to explain the law of falling bodies. The simplicity of the method, however, does not deny to it the susceptibility of affording ample demonstration of the law of gravity; namely, satisfactory and indubitable proof to the mind, in respect to the rate or intensity of gravity as proportioned to distance ; and whenever such indubitable proof is understandingly presented to the mind, whether by a numerical, a mathematical, or a geometrical process, if so be that ratio is the talisman that furnishes the proof, such proof may properly be called a demonstration. I speak thus with a view to the extreme simplicity in which demonstrations upon the most important points relative to the science of astronomy may be presented, and especially those relating to fundamental principles upon which the superstructure should be reared. Such is the case in regard to the law of falling bodies towards the earth, - from which it has been supposed that the law of gravity has been deduced; which law of falling bodies, and the consequent deduction of the law of gravity, depend upon a proper exposition of one of the most simple numerical formulas that can be devised, although the law of gravity may not be as simply or as beauti- 
fully developed and presented to the mind, by a consideration of the law of falling bodies, as by a consideration of Kepler's laws, (leaving out his elliptical hypothesis.) Nevertheless, as the method pursued for obtaining a knowledge of the law of gravity has been, by a consideration of the law of falling bodies; and as a proper consideration of the same will deduce a proper knowledge of the law of gravity; the labor bestowed upon the subject will be directed mostly to a deduction of the law of gravity from a consideration of the law of falling bodies; as thereby the popular methods may be quoted, and the popular modes or manner of investigation compared with my own.

Nor is truth or demonstration the less valuable in consequence of its simplicity, even though it may lie at the very foundation of the science of astronomy ; for if it were so, the truths disclosed by Kepler would be nearly valueless; for a more simple disclosure of the most important truths could scarcely be made, - a disclosure, too, developed and explained by the aid of our common numerical or Arabic characters, but from which, even yet, all physical truths worthy of the science of astronomy, have emanated.

Let the world, then, understand, - let mankind, who have supposed demonstrated truths to be beyond their comprehension, understand, - that the value of truth consists in the benefit which it bestows, - not in its complexity; and that demonstrable truths are vastly nearer to their comprehension than many have been led to believe. But notwithstanding the extreme simplicity and ease of comprehension of the demonstrations of the most important truths, (and especially in relation to the science of astronomy,) care should be taken that we do not extend the application of a demonstration beyond its legitimate province, lest we thereby deduce an inference which has no foundation in truth; which inference, when once engrafted into the science, is thenceforward implicitly received as truth. And such I have supposed to be the case in respect to the supposed demonstration, by Sir Isaac Newton, of Kepler's elliptical hypothesis, which has contributed so much to the fame of Sir Isaac Newton.

Kepler, in making calculations upon observations previously made by Tycho, (in which observations Kepler had great confidence,) came to the conclusion that the orbit of Mars was an ellipse, - or an oval, as he called it, - and he afterwards came to the conclusion that the orbits of all the planets were elliptical; and he found from observation that the motions of planets, at perihelion and aphelion, varied inversely as their distances from the sun varied; but he was unable to determine whether this was a general law throughout the orbit, but he supposed it to be; and 
if so, the radius vector would always describe equal areas in equal times.

Newton applied that proposition of Euclid which teaches that " triangles upon equal bases, and between the same parallels or equal altitudes, are equal to one another," which fully confirmed the truth of what Kepler had suggested, that the variation of the motion is in the inverse ratio to the variation of the distance ; and that too in all parts of the orbit; and consequently that the radius vector would always pass over equal areas in equal times. But that such demonstration goes to the extent of establishing Kepler's suggestions of elliptical orbits, perhaps none, on reflection, will seriously contend. The demonstration itself is only applicable to one of the ascertained laws of Kepler; namely, a law of motion depending for its phenomena upon the great physical law of gravity, and not at all upon his elliptical hypothesis ; for it will at once be seen that such demonstration applies as well to a circular orbit, whether centric or eccentric, as to an ellipse ; and that it will even apply to an orbit composed of a polygon of rectilinear sides, so long as the planet shall be performing one side of the polygon; for such is the condition upon which the proposition holds true, or upon which geometry is a science to be relied upon.

Such demonstration, then, has nothing whatever to do with the elliptical hypothesis, and should not have been used in its support.

\section{SECTION SECOND.}

It is possible, that from the days of Aristotle to the present, Physics and Metaphysics (especially so far as dialectics, or the proper modesiof reasoning, are concerned) have been treated of with a distinction not wholly consonant with the best philosophy. Those barriers, however, have been somewhat broken down by some modern philosophers and profound thinkers, among whom I will mention, by way of eminent example, Kepler and Kant, the philosophy of whom, taken in connection, and carefully analyzed and compared, would form the basis of a connected philosophy, which, if the true intent and meaning were not frittered away by false pretenders, or obscured by an occult philosophy which no mortal can comprehend, would not only become accessible to a vast portion of mankind who now grope in the darkness of philosophic error, in which it has been shrouded by many who claim to dispense science to the world, but would be worthy of that dignity of man to which science alone seems destined to elevate him. If, however, either of those champions in philosophy should, from too abstract a consideration of the sub- 
ject on which they may be particularly treating, deviate in any respect from those dialectic principles upon which all true philosophy should be based, and which is not properly the result of human logic, I nevertheless claim for mankind the right, the privilege to pause for consideration, rather than implicitly follow any file-leader to inexplicable conclusions.

Hence the monadology of Liebnitz with the incomprehensible consequences to which he would extend the hypothesis, however beneficial such hypothesis may be in disciplining the mind to a remarkable degree of astuteness, may, however, savor somewhat of that mysticism with which the Pythagorean theory of monads and dyads has been accused; there is not, perhaps, any philosophical objection to either Kepler, Kant, or Liebnitz, so far as they have been actuated in their researches by the Pythagorean philosophy, or manner of investigation, - notwithstanding certain conclusions or consequences may be imag. ined to result, which transcend the bounds of absolute investigation. It is said that the allegation of Pythagoras in respect to his philosophy, was conclusive; insomuch, that the phrase "he said so" was sufficient authority without any proof. But it is believed that the German philosophers, to whom I have referred, never aspired to be more than mortals while living, or to have divine honors paid them after death; this kind of ambition being incident to another person and nation; and hence, in what are termed our highest and best works on astronomy, the whole proof adduced in support of most material matters of which we should like to know, or be informed, often consists in that expression so obnoxious to science, that "Sir Isaac Newton found," \&c.; and it is somewhat surprising that, in this liberal age of inquiry, even the learned should be more willing to rely on such implicit faith, than to take the trouble to examine into the truth or error couched under the naked allegation. But it is yet to be hoped that, on serious consideration, infallibility will be considered an attribute which no mere mortal should impute to himself; and more especially, that the true dignity of man can never condescend to award such a meed of praise to a mer fellow mortal.

If Kepler, after an analogical course of reasoning upon the eternal fitness of things, by which he deduced certain physical laws, more beneficial to science than ever fell to the lot of any other mortal, eventuatly fell into an absurd conclusion, from placing too much confidence in the astronomical observations of Tycho, it is certainly no more than justice to him and to the world, to expose and correct such error. If Leibnitz became altogether too sensitive at losing the credit of what he esteemed his 
own discoveries in mathematics, it should be recollected that Mercator but just escaped a similar fate, and that poor Flamstead was totally ruined by the same means. And it may be quite doubtful, were Liebnitz to burst the cerement which shrouds him, and to come forth at this period of time, and behold the application of his discovery to what is, in the most emphatic sense, termed "the Newtonian System," (viz.' its application to the Newtonian theory and law of gravity,) whether he would not wish himself wholly freed from even the suspicion of having been the discoverer. If, however, his supposed discovery of an infinite series for the surface of the circle, is founded upon those errors which were and still are extant, and consequently lead to erroneous results, his fame, like that of any other mortal, should, nevertheless, stand upon its true merits, viz. that of the development of beneficial truths. Kant, with all his profundity of knowledge, doubtless applied his mind much more intensely, and reasoned much clearer upon trat compartment of philosophy which has in general been termed metaphysics, than upon what is generally called physics; hence, in the analyzation of the human mind, he was doubtless far more astute and better versed, than in those merely physical sciences, the apparent truths of which are but the results of the mind; and although he seemed well to understand the machinery of the human mind, by which a knowledge of physical science is developed, nevertheless, inasmuch as physical results were but secondary objects in his Critique of Pure Reason, and having written his Natural History of the Theory of the Heavens according to the Newtonian System, previous to his closer application to an analysis of the human mind, there, perhaps, can be no good reason why he might not have fallen into the same popular errors with others, in respect to the Newtonian theory, inasmuch as the subject was popular, and generally received as tantamount to demonstrated truth.

But after having so clearly and conclusively established his categories in respect to what forms the pure intuitions of the human mind, - viz. space and time, - as also those which are indispensable to our understanding, from which results and conclusions are deduced, it would seem almost incredible that a mind like his, with the elements of time and space so well considered and understood, should not have discovered the most important errors in the Newtonian theory and law of gravity; and more especially, as the great law of Kepler, that the "squares of the periods of the planets are as the cubes of their mean distances from the sun," is the most lucid exposition or commentary.of his category in respect to time and space, that can well be conceived. In the Keplerean laws, we find mathematical results corresponding 
with and proportioned to time and space, however varied such time and space may be; and for this very reason, it is perhaps the best corroborative evidence extant, in favor of a true development of the quadrature of the circle; inasmuch as an abstract mathematical consideration of the circle is destitute of time, as a comparative element. And hence I will again suggest, that peradventure, physics and metaphysics, have, in general, been considered in too distinctive a manner; for if, as taught by Kant, we cannot perceive anything except by means of certain original, necessary, unchangeable forms of thought; - if, to produce results, the categories must be applied to external objects, by which application they only become subject to error; if the demonstrative certainty of mathematics, the objects of which are space, time, form, \&c., or whatever quantity may be numerically expressed and proportioned, lies in the necessity of the forms of thought, and not in the range of error to which experience is subject; surely the most intimate connection exists between the physical and metaphysical world, so far at least as the human mind is concerned; nor could we, perhaps, readily distinguish this intimate connection from that of a unity of purpose in Him who created all things. If it be then the object of the Critique of Pure Reason, to mark out and define the categories, or transcendental ideas or pure intuitions, or the necessary and unchangeable forms of thought, and to direct their proper application to external objects with the view to avoid error in our results, the table of categories should not only be full and ample, but most explicitly explained or defined; and although they may all be comprehended in the four classes to which they are assigned by Kant, viz., quantity, quality, relation and modality, it may perhaps be questioned, whether Kant's division of the first class, or rather whether those categories assigned to the first class, - viz. unity, multitude and totality, - give a full and proper intimation of all that would seem to be comprehended under that head. To be sure, unity, multitude, and totality, are so far transcendental or intuitive, as not to depend on experience, or be subject to error from a misdirected course of reasoning; but when applied to those passive qualities or elements, in which all operations exist and take place, - viz., space and time, - which are as applicable to quantity, numerically expressed, as are the elements of force or motion, or even any tangible form whatever, - and inasmuch as quantity (however great or small) may always be taken or assigned at unity, and consequently, quantity, however great or small, may emphatically be the monad, or unity of Pythagoras, and the source of all numbers, or more properly the source of all ratio and proportion, (for I will not wholly discard the 
Pythagorean philosophy of numbers, from which our happiest and most beneficial results have been derived,) - it would seem as though what Kant has seen proper to call unity, might have been denoted by the term quantity, in which case, its adjuncts would have been denoted by those very expressions from which ratio and proportion (those indispensable elements in human wisdom) emanate or are deduced. 'Thus the adjuncts would have been a greater or a less quantity, or an increase or decrease of quantity, \&c., and I am unable to comprehend, that when applied to space or time, the idea of more or less, or greater and less, is less transcendental or intuitive, or that it is a less original or necessary form of thought, than that of multitude or totality; and if so, the former certainly would seem far better adapted to a proper application of the understanding, a priori, to external abjects or objects of research. If, then, the mind cannot have any real conception of quantity in the abstract, without having at the same time, a conception of greater or less quantity, I would certainly supply the place of unity, multitude and totality, by quantity, a greater quantity, and a less quantity. If quantity denoted by unity, or assumed at unity, be an abstract idea, (and perhaps abstractly, quantity cannot be considered otherwise ;) and if quantity, considered in the abstract, be numerically accompanied with, or be contained in the idea of more and less quantity, that is, that unity, however great or small, may be either increased or diminished; then, to be sure, that indispensable element to human . wisdom in the investigation of all phenomena, viz. that of ratio or proportion, lies as much in the necessity of the forms of thought, as that of time, space, quantity, \&c., and perhaps on this principle alone, rests the demonstrative certainty of mathematics.

I would suggest then, whether quantity may not properly take the place of unity, and whether its adjuncts, or those inevitable forms of the mind which accompany it, may not be more and less, or increase and decrease of any quantity which of itself is unity. In such case, quantity, - whether of force, motion, distance, space, time, \&c., - taken at unity, becomes the standard by which to proportion other quantities, whether they be greater or less; and this is to be done through the medium of ratio and proportion as evinced in the economy of numbers.

Thus the laws of force and motion, as displayed in time and space, are so adapted to the economy of numbers, exhibited in their powers and roots, or in that quality or principle in numbers which emphatically denotes and determines by mathematical precision, the ratio or proportion which exists between a quaniity taken at unity, and a greater or less quantity, - (which ratio or proportion, though existing in the mind as a necessary or 
primitive idea or thought, is only definitely determined by means of the powers and roots of numbers, - that unity of purpose will always be discernible in those laws which operate in space and time, if unity be made the standard by which to compare quantities; as well those of space and time, as of the operations in space and time. Thus in a system of planets revolving around the same central force, it is immaterial which planet we adopt as the standard by assuming its mean distance at unity, if we assume its period, its rate of velocity, its rate of motion, \&c., each at unity; for in such case, the elements of all the other planets of the system may readily be proportioned to each other, and to those of the standard planet, or that whose respective elements are called unity. Suppose the planets A, B and C, to revolve in centric orbits around the same centre of gravity; $\mathrm{A}$ at the distance $1 ; \mathrm{B}$ at the distance 2 , and $\mathrm{C}$ at the distance 4 ; the period of $A$ will be the square root of 1 ; that of $B$, the square root of 8 ; and that of $\mathrm{C}$, the square root of 64 ; and the rate of motion of $A$ will be the square root of 1 ; that of $B$ the square root of .5; and that of $\mathrm{C}$ the square root of .25 ; that is, in each case, the rate of motion will be inversely as the square root of the diameter, or the cube root of the inverse of the period. The distance of $\mathrm{C}$, being the square of the distance $\mathrm{B}$, the period of $\mathrm{C}$ will be the square of the period of $\mathbf{B}$; the rate of motion of $\mathrm{C}$ will be the square of the rate of motion of $\mathrm{B}$; so also, the force of gravity or attraction applied to $\mathrm{C}$, will be the square of that applied to B, \&c. And such will be the case whenever the distance of one planet is the square of the distance of another; and similar results will occur if the distance of one planet be the cube or fourth power of the distance of another planet of the same system.

In a centric orbit, what may be called the rate of deflection, is always inversely as the square of the distance, and is consequently the fourth power of the rate of motion; hence the square of the distance of the moon should have been referred to the rate of deflection, and not to the rate of force which caused the deflection; for the rates of deflection of the planets $A$ and $\mathrm{C}$, while passing over equal space, will be inversely as their distances; but while passing equal time, they will be inversely as the squares of their respective distances. Hence, if the force be inversely as the distance, their deflection, while passing over equal space, will be as the force; but while passing over equal time, will be as the square of the force. But if the force be inversely as the square of the distance, their deflection while passing over equal space, will be as the square of the force; and while passing over equal time, will be as the force; which in nowise cor- 
roborates the law of falling bodies. For if a body fall from a state of rest, the amount of fall in any given time, is the square of the whole amount of force applied from the commencement of the fall; for the force applied is as the time. Hence, if, in given time, the amount of fall of one body from a state of rest be sixteen times that of another, the force that urged it must be four times as intense as that which urged the other in its fall; otherwise the sum of all the spaces passed over at the end of any given moment, could not be the square of the sum of all the moments of time from the commencement of the fall. So if two bodies, $M$ and $N$, fall from a state of rest at the same time, and at the end of any given moment, $M$ shall have fallen four times as far as $\mathrm{N}$, the constant force that urged $\mathrm{M}$ will have been twice as great as that which urged $\mathrm{N}$; but if $\mathrm{M}$, at the end of any given moment, shall have fallen sixteen times as far as $\mathrm{N}$, in such case, the force that urged $M$ will have been four times as great as that applied to $\mathrm{N}$. Let us now endeavor to apply these principles to the movement of planets in their orbits.

In a centric orbit the rate of motion is always inversely as the square root of the distance: hence, if the rate of motion, is the square root of the force, then the force is inversely as the distance; for the simple reason, that the inverse of the distance is the square of the inverse of the square root of the distance. Hence, in a centric orbit, because the rate of motion is inversely âs the square root of the distance, if the force is inversely as the square of the distance, then the rate of motion must be the fourth root of the force; - for the simple reason, that the inverse of the square root of the distance is the fourth root of the inverse of the square of the distance. So in an eccentric orbit, in the points of mean motion to which we must refer the rate and progress of the motion, the motion is, of necessity, the inverse of the square root of the mean distance, as much as it is in a centric orbit; else in a system of planets, the squares of their periods cannot be as the cubes of their mean distances from the sun. Hence in the points of mean motion, the motion will be the square root of the force, if the force is inversely as the distance. But if the force be inversely as the square of the distance, then in the points of mean motion, the motion will be the fourth root of the force. And here it may be proper to pause for a moment, examine the original chart, and get our latitude and longitude.

One of the famous discoveries of Kelper was, that the motion of a planet revolving in its orbit, varies in an inverse ratio as the distance varies. This phenomenon only appertains to eccentric orbits, and is of the utmost importance in the explanation of 
the laws of force and motion, as applied to such orbits; and had not its force and effect, its spirit and efficacy, been frittered away, and but the shadow been retained in lieu of the substance, by those who succeeded Kepler, we might, long since, have been reaping the benefits which the discovery of that principle seemed so well calculated to bestow on science. Yes, frittered away, - until at length we find in place of it, in some modern works on astonomy, the following, quoted as Kepler's law, namely, "The areas described by the radius vector of a planet are proportional to the times." I am aware that this rendering of the law is in accordance with Sir Isaac Newton's notions of its importance to the science of astronomy; for Mr. Vince says that Kepler, after the discovery of his said law, concluded, of course, that the radius vector would describe equal areas in equal times, but was unable to prove that it was so throughout the orbit; and that this supposition of Kepler's was what first suggested to Newton the idea of his Principia; and that Newton proved or demonstrated Kepler's supposition to be true; and the extended consequences, (for good or for evil,) flowing from said demonstration, (though of no use whatever to the science of astronomy,) I have endeavored in part to exhibit. Thus the shadow was not only received for the substance, but the substance was perverted, made void, and useless; insomuch, that in lieu of its being a variation of the motion of a planet in the inverse ratio as the distance from the sun varies, it has been more commonly taught, or, at least, received, that the motion, or rate of motion of the planet, is inversely as its distance; from which the grossest errors have arisen and been inculcated in respect to the laws of force and motion. For there is not a simpler or plainer principle in respect to the laws of motion of the heavenly bodies, than that the rate of mean motion of a planet is inversely as the square root of the mean distance, and that when it revolves from those mean points, the motion varies inversely as the distance varies. If it were not so, the squares of the periods of a system of planets could not be as the cubes of their mean distances from the sun; for the very condition of this law is based upon the principle, that the mean motion of a planet is the inverse of the square root of the mean distance; as any one will discover upon the slightest examination.

But if the motion of a planet were inversely as the mean distance, the squares of the periods of a system of planets would be as the fourth powers of their mean distances. And in such case, according to Newton's law of gravity, namely, " that force or gravity is inversely as the square of the distance," the force would always be the square of the motion in any part of the 
orbit; and upon this hypothesis has the base of astronomy been laid. And hence I will allege that the only principle upon which the hypothesis that the force is inversely as the square of the distance, could be sustained, would be, that the motion of a planet in all parts of its orbit, (whether centric or eccentric,) is inversely as the distance from the sun; in which case, if the force were. inversely as the square of the distance, it would then, of course, be the square of the motion. In such case, if the distance were 1 , the period would be 1 ; if the distance were 2 , the period would be 4 , in lieu of being 2.8284 ; if the distance were 4 , the period would be 16 , or double what it really is. That is, the period would always be the square of the distance, in lieu of being the cube of the square root of the distance.

Let us exemplify the subject in the following manner: Whenever it is found convenient or proper to compare the elements of bodies revolving around different central forces, as of satellites about two primary planets, if the attractive power of one of the central forces be assumed at unity, and the other central force be numerically proportioned to unity, we may then state the law thus, namely, the square of the period is the cube of the distance divided by the force of the central body; which version will apply equally well when there is but one central body about which several bodies revolve, but is quite convenient when there are two central bodies. Thus, suppose two primary planets whose attractive powers are as 1 to 4 , each having a satellite revolving around it, the satellites being at the same or at equal distances from the centres of their respective primaries. Let the primary whose force is 1 be denoted by A, and let the distance, and consequently the period of its moon, be called unity or 1 ; and let the primary whose force is 4 , be denoted by B. Now because the mean force is the square of the mean motion, and consequently is inversely as the distance; as the period of A's moon is 1 , so the period of B's moon will be $.5 ;$ and in either case, it will be perceived that the square of the period is the cube of the distance divided by the force of the central body; which law will be general, whatever be the distances of the respective satellites.

If it be true, - as found by Euler, LaGrange, and other eminent astronomers and mathematicians, so far, at least, as could be ascertained from observation and calculation, and even to that degree of exactness which induced the latter to base certain astronomical tables upon the hypothesis, and of which he afterwards remarked that he saw no occasion for altering or changing his opinion in respect to the hypothesis, - that the power of a planet to attract its satellite is conjointly as the bulk or mag- 
nitude directly, and its distance from the sun inversely; - then if two primary planets of equal magnitudes revolve about the sun, one of them at the distance 1 , and the other at the distance 4, each having a satellite revolving around it, each at an equal distance from the centre of the primary, - the period of the satellite of that planet furthest from the sun would be double the period of the satellite of the planet nearest the sun; and in this ease, twice the motion would require quadruple the force; and in either case, the square of the period would be the cube of the distance divided by the force of the central body. Kepler's law may be properly described thus: The period is the square root of the third power of the distance, - or the distance is the cube root of the square of the period, - divided by the force. Let us assume the law of gravity to be inversely as the distance, and let three satellites, A, B, and C, revolve around one and the same primary, $A$ at the distance $1, B$ at the distance 2 , and $\mathrm{C}$ at the distance 4 . Then the period of $\mathrm{A}$ will be 1 , and its rate of motion 1 ; the period of $B$ will be 2.8284 , and its rate of motion .707106 ; the period of $\mathrm{C}$ will be 8 and its rate of motion .5. The rate of force exerted on $\mathrm{A}$ will be 1 , on B .5, and on C .25; thus fulfilling the law that twice the motion requires four times the force to retain the planet in its orbit. Suppose now the Newtonian law of gravity to prevail in respect to those bodies, namely, that the force exerted upon $\mathrm{C}$ is four times that exerted upon $B$, and sixteen times that exerted upon A. In such case, it would seem that inasmuch as $B$ receives quadruple the force that $\mathrm{C}$ receives, the motion of $\mathrm{B}$ should be equal to that of $A$. And inasmuch as $B$ receives but one fourth the force that $A$ receives, the motion of $B$ should be only equal to that of C. And whether in such case B is placed in a dilemma, I must leave the world to judge.

\section{SECTION THIRD.}

Perhaps no part of astronomy has caused the philosopher and mathematician more perplexity than that arising from what is usually termed the deffection of a planet from a tangent to its orbit, accompanied as it has been with the supposition of its identity, in its mathematical construction, with the simple and well known law of falling bodies near the earth, and from the attempts to reconcile and harmonize what is called deflection from a tangent, with the legitimate elements for deducing the laws of force and motion, namely, time, distance, motion and gravity. Hence it is not passing strange that the world should have received the doctrine that the law of gravity is inversely as 
the square of the distance; having taken as their guide at the setting out, a wholly factitious element in the laws of force and motion, supposing it to possess a quality which it does not, namely, identity in its mathematical or numerical construction with the law of falling bodies near the earth. The error, however, should not have been committed, as I will endeavor to show; even though deflection is identical with the law of falling bodies, and for that purpose, in the present section, I will suppose deflection to be identical with the law of falling bodies, although I think it demonstrable that it is not.

For the purpose of exhibiting Newton's method of assigning the law of gravity, his method of investigation, and his result, (which has generally been supposed to be in conformity with the law of falling bodies,) I will quote from Rees' Cyclopædia, Vol. XXV, article Moon, wherein, after giving the magnitude, motion, density, \&c., compared with the earth, together with the irregularities of its motions, \&c., the author proceeds as follows: "The irregularities above enumerated and some others of a similar kind, have been urged as objections to the Newtonian theory of gravity, though they were anticipated by the illustrious author, who not only evinced their consistency with it, but suggested the explication of them which might be deduced from that theory properly understood and applied." Mr. Rees, after quoting from Mr. Vince's astronomy on the same subject, proceeds : "Mr. Euler also retracted his own erroneous opinion in deference to the judgment of Mr. Clairaut, and concurs with him in doing ample justice to the Newtonian theory," and observes that "others, and particularly Mr. Machin and Mr. Frisi, have prosecuted a similar investigation of this theory and contributed to establish it." Mr. Rees then proceeds in this very remarkable manner : "It would not be consistent with the limits nor the nature of this work, to investigate, by tedious and elaborate processes, of an analytical and geometrical kind, the various equations that have been explored for the illustration of these laws. Much has been done in this way by several learned mathematicians, and of late by Professor Vince, who is eminently qualified for the undertaking; and we shall therefore refer the reader who may be desirous of further information, and who has no access to a variety of other publications, to the second volume of Vince's Complete System of Astronomy, Chapter XXXII." Mr. Rees proceeds : "We shall, however, in this place, introduce a general view of the Newtonian theory of gravity, as it is applied to the solution of the investigations of the moon's motions." "We have already, under the article Gravitation, iliustrated and confirmed the Newtonian theory of gravity, as it regards the 
moon and other planets; but as the subject is of importance, and as it is immediately connected with what follows, we shall here give a concise statement of the leading facts by which the identity of the centripetal force as it respects the moon, and that of gravity, was originally explained and established, referring for a more detailed account, to the article just cited." "It is well known and universally allowed that the planets are retained in their orbits by some power which is constantly acting upon them; that this power is directed towards the centres of their orbits; that the efficacy of this power increases upon an approach to the centre, and diminishes by its recess from the same, and that it increases according to a certain law, namely, that of the squares of the distances, as the distance diminishes; and that diminishes in the same manner as the distance increases. Now by comparing this centripetal force of the planets with the force of gravity on the earth, they will be found perfectly alike. This we shall illustrate in case of the moon, the nearest to us of all the planets."

"The rectilinear spaces described in any given time by a falling body, urged by any powers, reckoning from the beginning of its descent, are proportionable to those powers. Consequently the centripetal force of the moon, revolving in its orbit, will be to the force of gravity on the surface of the earth, as the space which the moon would describe in falling any little time, by her centripetal force towards the earth, if she had no circular motion at all, to the space which a body near the earth would describe in falling by its gravity towards the same." "By a very easy and obvious calculation of these two spaces it will appear that the

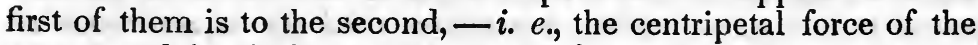
moon revolving in her orbit is to the force of gravity on the surface of the earth, - as the square of the earth's semi-diameter to the square of the semi-diameter of her orbit, which is the same ratio as that of the moon's centripetal force in her orbit to the same force near the surface of the earth. The moon's centripetal force is therefore equal to the force of gravity. These forces consequently are not different, but they are one and the same; for if they were different, bodies acted upon by the two powers conjointly, would fall towards the earth with a velocity double to that arising from the sole power of gravity." "It is evident, therefore, that the moon's centripetal force, by which she is retained in her orbit, and prevented from running off in tangents, is the power of gravity of the earth extended thither."

Mr. Rees, then, after the manner of others, supposes that the moon is drawn from its tangent towards the centre of the earth about $15 \frac{1}{2}$. Paris feet in a minute of time, which is the same 
distance a body near the surface of the earth will fall in one second of time, and proceeds thus: "But this force (as applied to the moon) being known from the elliptical figure of her orbit to be reciprocally proportional to the square of the distance, would impel the moon, supposed to be at the surface of the earth, through a space equal to $60 \times 60 \times 15_{12}^{1}$ feet in one minute." "But bodies impelled by the force of gravity fall near the surface of the earth through the space of $15_{12}^{1}$. Paris feet in one second, and the spaces being as the squares of the time, through $60 \times 60 \times 15_{\frac{1}{12}}^{\frac{1}{2}}$ in a minute. Consequently, as the force by which the moon is retained in its orbit, and the force of gravity, produce the same effects in the same circumstances; they are the same forces."

This then is the law of gravity, as promulgated by Sir Isaac Newton, by which the heavenly bodies move in their eternal rounds. I will therefore give a short version of the foregoing method to suit my own purposes and my own method of investigation, and will in due time, enter upon a confutation of his deductions, and upon the support of my own. Thus a body commencing its fall near the surface of the earth will, in the first second of time, (which may be called the first moment,) fall about $15_{12}^{\frac{1}{2}}$ Paris feet, which may properly be called one space; in the second moment the body will fall over three like spaces; over five like spaces in the third moment, and so on; the spaces increasing with the odd numbers in their order. Hence if we set down all the common numbers in their order, as $1,2,3$, 4 , and so on, ad infinitum, and extract the square root of such series, taking figure 1 at the left hand as the whole of the first period in the process of the extraction, then the square root of such series will be expressed by placing figure 1 over each odd number in the series, in which case we shall have a very convenient formula whereby to express the law of falling bodies near the earth ; in which, the square root of the series constantly expressed by figure 1 , will denote the individual and equal moments, (and also the equal forces in those moments,) in which the body shall have been falling at the end of any given moment. The sum of all the odd numbers of the series will be the whole amount of spaces over which the body has fallen at the end of any given moment, which will be the square of the sum of all the moments or of the forces in those moments in which the body has been falling; and the even numbers in the series will denote the acquired motion, or the rate of motion at the end of any given moment. The moon revolves about the earth in an orbit 60 times as far from the centre of the earth (or centre of gravity) as the surface of the earth is; and we will suppose it to be found 
from observation and calculation, that the fall of the moon from a tangent to its orbit, towards the centre of the earth, is one space, or $15_{\frac{1}{2}}^{\frac{1}{2}}$ feet, in 60 minutes, and hence that the fall of the moon, at her distance from the earth, is but $\frac{1}{3600}$ part as rapid as that of a body at the surface of the earth.

And from premises like these the deduction has been drawn by Newton and endorsed by others, that the force is inversely as the square of the distance; notwithstanding the supposed identity shown between the centripetal force which retains the moon in its orbit, and the law of gravitation at the surface of the earth which causes bodies to fall and to accelerate their motion during the fall. And hence it has been concluded that the force of the earth's attraction at its surface is 3600 times as great as at the distance of the moon, when in fact it is but 60 times as great; as I propose to show by a very simple process. And had Clairaut and Euler but taken a common sense view of the subject, (having the elements all before them,) it would not have been necessary for them to have retracted their rational doubts as to the Newtonian law of gravity, or to have resorted to what is called the problem of the three bodies, or any other mysterious problem, for the purpose of accounting for the motion of the moon's apogee, in corroboration of a law as simple as it is universal.

In the present investigation of the subject, I will make use of the element of time, in the abstract, as that of equal moments, in lieu of our arbitrary division, as that of seconds, minutes, \&c., each of which moments being assumed at unity, or as a unit of time, may be rationally and readily compared with the other requisite elements in the investigation, each of which will be used in the abstract, and each assumed at unity; and such method is as applicable to the law of falling bodies as to an investigation of planetary motion. Now, "the squares of the periods of the planets are as the cubes of their mean distances from the sun" - is emphatically the great astronomical chronometer from which the most simple and rational deduction can be drawn in respect to the law of gravity, and its application to planetary motion. Thus if unity be made, (as it emphatically is,) the base or centre of all ratio, then, in respect to any one planet of a system, if one of the elements used in any investigation be assumed at unity, each and every other of the elements must be assumed at unity, else the square of the period will not be the cube of the distance.

Now, our great object of inquiry is, what is the rate or ratio of the force of gravity to that of distance from the centre of gravity? This then naturally suggests, as the first or standard element, that 
of passive distance in space, which has nothing whatever to do in the performance of the operation. Nevertheless, a ponderous body is not passed over, or made to describe a given quantity of space, except in some given quantity of passive time; hence passive time may properly be regarded as the second element to be considered.

The third element is that of the given space to be passed over or described in a given time at a given distance, and is consequently a passive element.

The fourth element is that of motion, and is of itself an effect, an operation, being the phenomenon observed; the rate of which is to be proportioned to the rate of motion at a different distance.

The fifth element is that of the force of gravity which causes the phenomenon, or passage of the body over the given space, or which controls its passage over the given space, the rate of which is to be proportioned to the rate of force at a different distance, and is to be deduced from a proper consideration of the other elements, and more especially from a proper consideration of the different rates of motion, (or of the observed phenomena,) at different distances from the centre of gravity. And whether we attempt to deduce the rate of force or gravity as proportioned to distance, from a consideration of two or more planets revolving in their orbits at different distances from the sun; or from a consideration of two or more bodies commencing their fall towards the earth at the same time, but from different distances from the centre of the earth; that planet, or that body whose distance is assumed at unity, must necessarily have each and every one of its elements assumed at unity, by which the elements of the other planet or body may be compared and proportioned, as with ratio in unity. Notwithstanding, if two planets revolving round the sun at different distances, or if two bodies commencing their fall at the same time from different distances from the centre of the earth, in which each and every element must be assumed at unity for that planet or falling body whose distance is assumed at unity; nevertheless, as what is termed the amount of deflection of a planet from a tangent to the orbit (even in any little time) while revolving in the orbit, is not the same, or not equal in amount to what the rectilinear fall of a body from a state of rest towards the centre of gravity from the point of contact of the tangent and orbit, would be in the same given time, however small, (although they were assumed to be equal by Sir Isaac Newton,) such difference will cause a much greater dissimilarity between the methods of 
deducing the law of gravity from a consideration of two or more planets, and the law of falling bodies,' than would otherwise exist. But notwithstanding the amount of deflection of a planet from a tangent, even in any little time, is equal to the versed sine of the arc described by the planet in such little time, yet such versed sine, being greater in amount or not of the same amount of space, as would be described by a body falling from a state of rest towards the centre of gravity from that distance in the same little time, would seem very strongly to suggest that a like discrepancy must exist between any numerical development of the law of falling bodies, and that which is generally supposed to give a rational approximation of the ratio of the circumference of the circle to the diameter, as would exist between that of falling bodies and the deflection of a planet from a tangent to its orbit. Nevertheless, either of these methods should have led to the conclusion that the force of gravity varies inversely as the distance varies; and it may not be amiss here to give some few hints as to the discrepancy above named; that the mind may readily make the application in respect to such discrepancy.

Conceive then the planets $\mathrm{A}$ and $\mathrm{C}$ revolving in centric orbits about the sun; $\mathrm{A}$ at the distance 1 , and $\mathrm{C}$ at the distance 4 ; and conceive a straight line to pass from the centre of the sun, intersecting both orbits, called the intersecting line; let a tangent to each orbit pass from the intersecting line in the direction in which the planets are to revolve, - and let the two planets be conceived to start from the intersecting line at the same instant of time.

Then if A shall have passed ever so small a distance in its orbit, from the intersecting line, $\mathrm{C}$ will have passed one half the distance that $\mathrm{A}$ has. Now some small distance from the intersecting line on the orbit of A, may be called a unit of space; and the time in which $\mathrm{A}$ is describing it may be called a unit of time; hence in one unit or moment of time, $\mathrm{C}$ will describe half a unit of space, or in two units or moments of time, will describe one unit of space; and when A has passed over one moment or one unit of space from the intersecting line, the amount of its deflection from the tangent will be called unity or 1. And hence, if such deflection be governed by the law of falling bodies, when A has passed over two units of space, its amount of deflection will then be 4 , or four times that at the end of one space. But as the orbit of $\mathrm{C}$ is four times as dilate as that of the orbit of $\mathrm{A}$, if the law of falling bodies were to prevail according to the hypothesis of Sir Isaac Newton, then, when $\mathrm{C}$ has passed one unit of space, its amount of deflection would be just one fourth 
of that of $\mathrm{A}$ at the end of one space, and when $\mathrm{C}$ has passed over one moment or unit of time, its amount of deflection would be just one sixteenth that of $A$ when $A$ has passed over one unit of time. If such be the case, (and it is so, excepting the variation,) then at the end of equal time, the force applied to $\mathrm{C}$ is (in the whole amount) one fourth of that applied to $A$ in the same time, for the reason that it would produce one sixteenth of the deflection in $C$ that the force applied to $A$ gives in the same time. And when $C$ and $A$ have passed over equal spaces, the deflection of $\mathrm{C}$ will then be one fourth that of $\mathrm{A}$, which, if strictly true, would perfectly agree with the law of falling bodies. It is manifest; then, that there is a resemblance between deflection and the law of falling bodies; and were they identical, then the law of deflection would be sufficient to prove conclusively that the rate of force or gravity applied to $\mathrm{C}$, is one fourth of that applied to $A$, which rate of force properly refers to time, viz., a continuation of the force long enough to make up a given amount. Deflection, however, does so far resemble the law of falling bodies, that those who undertook to prove them identical, and to deduce the law of gravity therefrom, should have referred the square of the distance to the amount of deflection in time, in lieu of referring it to the rate or intensity of the force of gravity ; for the rate of deflection in time would then have been assumed as the inverse of the square of the distance, and as the square of the rate of gravity directly, as in the case of falling bodies. If then deflection were identical with that of falling bodies, then, in either case, the amount of deflection or fall from the beginning would always be the square of the whole amount of force expended during such deflection or fall.

In such case, the amount of deflection being as the square of the force directly, and inversely as the square of the distance, the rate of force must necessarily be inversely as the distance, as the same is determined by the law of falling bodies. Here then rested the Newtonian error, in referring the square of the distance to the force, instead of referring it to the fall or deflection in time, to which it manifestly relates. Thus, to refer again to the planets $\mathrm{A}$ and $\mathrm{C}$, and suppose deflection and the law of falling bodies to be identical, $\mathrm{A}$ would receive twice the amount of force that $\mathrm{C}$ would, while passing over equal space, because the deflection of $\mathrm{A}$ would then be quadruple to that of $\mathrm{C}$; and $A$ would receive quadruple the amount of force that $C$ would while passing over equal time, because the deflection of $\mathrm{A}$ would then be sixteen fold that of $C$. Hence $A$ receives double the force in half the time; or $\mathrm{C}$ receives half the force in double the time; the consequence of which is, that in equal time $A$ receives 
four times the amount of force that $\mathrm{C}$ does; or $\mathrm{C}$ receives one fourth of the amount of force that $A$ does in equal time; hence if the time of $\mathrm{C}$ be 4 , and the time of $\mathrm{A} 1$, they will then each have received a like amount of force; proving that the rate of force is inversely as the distance; that the rate of force applied to $\mathrm{A}$ is fourfold that applied to $\mathrm{C}$; and as the time of the period of $\mathrm{C}$ is eight times that of $\mathrm{A}$, hence $\mathrm{C}$ will receive, in one of its entire revolutions or in the time of its period, double the amount of force that $A$ will receive in the time of A's period. But if the rate of force were inversely as the square of the distance, then $\mathrm{C}$, in the time of its period, would receive just half the amount of force that $A$ would receive in the time of $A$ 's period. If the amount of deflection of $\mathrm{C}$ were just four times as great in two units of time as it is in one, (in accordance with the law of falling bodies, ) then the amount of C's deflection in one unit of time would be the square of the amount of C's deflection in one unit of space. The distance of $\mathrm{C}$ being 4, its period is consequently 8 , otherwise it would not fulfil the Keplerean law; hence if the distance of another planet be the square root of the distance of $\mathrm{C}$, the period of such planet will necessarily be the square root of the rate of motion of $\mathrm{C}$; and the same is true of its rate of force or gravity; and also of its deflection in time. And similar will be the case whenever the distance of one planet shall be the square root of the distance of another planet of the same system; for such result only will fulfil the great law of Kepler.

Hence the distance of a planet divided by the rate of its mean motion gives the time of its period; or the distance divided by the time of the period, gives the rate of mean motion. Hence the mean distance is always the square of the reciprocal of the mean motion; or the mean motion is the reciprocal of the square root of the mean distance, or of the cube root of the time of the period. We find then these proportions to prevail between the rate of motion and the time of the period, viz., when the distance is 1 , the rate of motion is equal to the period; when the distance is 2 , the rate of motion is equal to 1-4th of the period; when the distance is 3 , the rate of motion is equal to $1-9$ th of the period, \&c.; or in other words, the distance divided by the time of the period gives the rate of motion ; and the rate of motion is always, and by every consideration which may be given to the subject, inversely as the square root of the distance ; for this it is, which establishes the phenomena according to Kepler's law. Hence if we take three planets, A, B and C, - A at the distance $1, \mathrm{~B}$ at the distance $2, \mathrm{C}$ at the distance $4,-$ the time required for $B$ to pass over a space equal to the circumference of 
A's orbit, will be equal' to the square root of B's distance, viz., the square root of 2 ; and the time required for $C$ to pass over a like space will oe equal to the square root of C's distance, viz., the square root of 4 ; the motion, or rate of motion of each planet, being the reciprocal of the square root of its own distance. And because the rate of motion is inversely as the square root of the distance, hence if the rate of the force of gravity be inversely as the square of the distance, the rate of force or gravity will be the fourth power of the rate of motion. But if the rate of force or gravity be inversely as the distance, then the rate of force or gravity will be the square of the rate of motion, in accordance with all we know in respect to the laws of force and motion as resulting from a consideration of mechanics or dynamics, as also with well established facts which have long been assented to by mathematicians and astronomers, in matters wholly analogous and corroborative: as that twice the motion requires quadruple the force, whether it be the result of an impulsive force or of that constant and uniform force which retains a planet in a centric orbit; or produced in any other manner in which motion may be conceived to be the result of force. In respect to the difference in point of identity between deflection from a tangent, and the law of falling bodies, inasmuch as I shall have occasion hereafter to refer to some direct and conclusive evidence on this point, I will defer the same for the present and proceed to a short consideration of the law of falling bodies, from which the law of gravity may also be deduced.

In a consideration of the law of falling bodies, the force applied is to be considered as constant and as uniform as the time in which the force is applied; and the whole amount of force applied while the body is describing a given space, may be divided into as many equal parts as the whole amount of time is divided into in which the body is describing the given space.

The time required for a body to fall from a state of rest over a given space (one unit of space) is as the distance, by all determinations of the law of falling bodies.

Hence the rate or intensity of the force bears the same ratio or proportion to the distance that it does to the time. It is as much less or more than the distance as it is less or more than the time. If the rate or intensity of the force which urges a body over a given space, or unit of space, be inversely as the distance, it is also inversely as the time. If it be inversely as the square of the distance, it will also be inversely as the square of the whole time, - thus fulfilling the whole law in respect to the mechanical powers.

If $\mathrm{A}$ and $\mathrm{B}$ are to fall from a state of rest at the same time, $\mathrm{A}$ from the distance 1 from the centre of gravity, and B from the 
distance 60 , (each body to describe a like amount of space, namely, a unit of space,) B, or the body at the distance 60 , will require sixty-fold as long time as $A$, or the body at the distance 1. Hence if the labor to be performed, (namely, the unit of space to be described,) be at sixty-fold the distance from the centre of gravity, (or centre of force,) the force at that distance is proportionally so much less than at the distance 1, that sixty-fold the time is required that would be required at the distance 1 , for performing the same amount of labor.

If, then, we proportion the rate or intensity of the force to the whole amount of the time passed over, we thereby proportion the rate or intensity of the force to the whole amount of the distance from the centre of gravity. The space then to be described, or the labor to be performed, being unity, the length of time determines the distance, or the distance determines the length of time. And until we are able to obtain some kind of evidence that the great, eternal, and universal law, - that the intensity of the force is diminished as'the whole time is increased, or that the rate or intensity of the force must be increased as the whole time shall be diminished, in order that the task or labor shall be performed,has been altered, we had better abide by the law as it was in the beginning, is now, and ever shall be. The following numerical formula is in accordance with those generally given for denoting or explaining the law of falling bodies towards the earth, - and the same may also serve to indicate how extremely simple those demonstrations are, and always have been, which lie at the foundation of true science, and have rendered the greatest benefits; and hence, important demonstrations are not enveloped in a cloud of darkness beyond the ken or sagacity of most mortals to penetrate; and whenever what may be alleged to have been demonstrated is so clothed, it is to be feared that it is but the attempt to equate error to correspond with truth, which must necessarily require a mysterious process. It will be seen, also, that the following formula is only intended to denote the numerical law of a falling body in reference to the manner in which time is apportioned to any given amount of space described or passed over by the body in its fall from a state of rest, without regard to distance from a centre of gravity, and consequently without regard to any rate, intensity or efficiency of force by which the body should be urged, and without intention to ascertain the rate or intensity of gravity as proportioned to distance from a centre of gravity; for, short of a comparison of certain elements of two falling bodies, the rate of gravity could not be proportioned to the distance.

In accordance with the popular formula above named, the upper series, composed of number 1 , repeated, may be called the 
uniform series, and is used to divide the whole amount of time in which a body is falling over a given space, into equal parts or moments, as seconds, for instance.

The second column, composed of the odd numbers in their order, may be called the accelerating series, - the sum of which odd numbers at the end of any given moment of the time, will always be the square of the number of moments of time in which the body has been falling; or, in other words, the amount of space described or passed over at any given instant of time from the commencement of the fall, is the square of the whole time that has been passed over from the commencement of the fall. Thus the whole space passed over during the amount of time allowed for the fall, is, by the series of odd numbers, divided into as many equal parts (called spaces) as will make the square of the number of equal moments into which the whole time is divided; the first space, or that denoted by unity, or figure 1, being the amount of space which the body will describe in the first moment of time; and the law, numerically expressed, is, that the body will describe three times the space in the second moment that it does in the first, - five times in the third moment, and so on.

Another part of the law, in respect to a single falling body, is numerically expressed by the even numbers in their order, denoting the rate of velocity or motion acquired at the end of any given moment of the fall. The acquired motion at the end of any given moment of time, being sufficient, without further force applied, to carry the body over one more space in the next succeeding moment than it passed over in such given moment. But by the aid of the constant force applied, the body will pass over two more additional spaces in each successive moment, thus increasing the number of spaces as the odd numbers increase; and hence we find that the acquired motion is as active as the force is efficient.

A formula, however, designed to express numerically the law of a falling body; may always be most properly expressed in two series or lines of figures, namely, the uniform series, or that expressed by the constant figure 1, and the series of natural numbers; figure 1 occurring only once in each odd number of the series of natural numbers. Then the uniform series to any extent will be the square root of the series of natural numbers to the same extent, in case we make figure 1 , in the natural numbers, the first periodin the process of extraction; and in such case the even numbers in the series of natural numbers, (namely, those figures which denote the rate of acceleration,) will always come out as a remainder in the process of extracting the square root, or uniform series 
The formula, with the uniform series carried or extended to eight places, may be thus :

$$
\begin{array}{l|llllllllllllllll}
1 & 1 & & 1 & & 1 & & 1 & 1 & & 1 & 1 & 1 & \\
1 & 2 & 3 & 4 & 5 & 6 & 7 & 8 & 9 & 10 & 11 & 12 & 13 & 14 & 15 & 16
\end{array}
$$

Now it is manifest that in calculating the law of the fall simply of an individual body from a state of rest, - in which case distance from the centre of the earth or centre of gravity is not material, for the reason that the law, numerically expressed, will be the same, whether the force be more or less, (so that it be uniform, - - we may always consider the distance from the centre of gravity to be unity, whether it be at the surface of the earth or at the distance of the moon; and that it is only when the fall of two bodies, commencing their falls at different distances are compared, that it becomes necessary to assume one of the bodies at unity, and the other at some other distance, either greater or less than unity, as we may choose.

The comparison, however, may be made by means of two simple formulas, as in case of the foregoing; the first, or that preceding the double line; being composed of two figures only, each denoting unity, the upper figure of which may denote either unity of distance from the centre of gravity, unity of time in which the fall or operation is to take place, or unity of force or gravity, namely, the whole amount of force or gravity expended on the body during the operation, namely, during its fall over the given space; and the lower figure in the first formula denoting the given space to be described or passed over, namely, a unit of space, or the same amount that is to be described or passed over in the second formula, or that succeeding the double line.

The uniform series in the second formula denotes as many unities of distance from the centre of gravity, or as many unities of time in which the operation is to be performed, as there are figures in the series; and the whole amount of force of gravity (whatever such amount may be) is divided into as many equal parts as there are figures in the uniform series; namely, into as many equal parts as there are units of distance, or units of time; and the odd numbers in the lower series of the second formula are used to divide the given space to be described (namely, a unit of space, or the same amount denoted by the lower figure in the first formula) into as many equal parts as there are units contained in all the odd numbers from 1 to 15 inclusive, namely, 64 , or the square of the units of distance, or of the time of the fall, as also of the whole amount of force expended during the fall, or, if we please, of the sum of the equal parts into which the 
whole amount of force is divided by the uniform series; and then 1-64th of the space will be described while 1-8th of the foree is being expended; 3.64ths more of the space will be described while another 1.8th of the force is being expended, and so on; the amount of space described increasing by the odd numbers, while the force is uniform. Now the intensity or rate of force employed in the second formula is less by some given ratio than that employed in the first formula, - that employed in the first being unity ; and the ratio of the intensity of the force employed in the second formula, to that employed in the first, is what we are seeking for, which, of course will give the ratio of the rate or intensity of the force to that of the distance from the centre of gravity. Now in either formula, the last 15-64ths of the unity of space will be described, while an equal amount of force is being expended, as while the first 1-64th of the space is being described; for the law is the same in both formulas. But it is too serious a question to be lightly determined, whether the amount of force expended upon the body in the first formula, while passing over the first 1-64th of the given space, or the last 15-64ths of the given space, shall be eight-fold, or only equal to the amount of force expended on the body in the second formula while passing over the first $1-64 \mathrm{th}$, or the last $15-64$ ths of the given space, or unit of space; namely, whether effective force shall perform the labor, or whether 7-8ths of the labor shall be denied to the operation of force, and credited to passive time; thereby assigning or requiring the same amount of force to be expended on the body in the first formula while describing the first 1-64th part of the unit of space to be described, as will be expended on the body in the second formula, while describing the whole of the unit of space; which is readily perceived to be the case, if the rate of force varies inversely as the square of the distance; while if the rate of force varies inversely as the distance, eightfold the amount of force will be expended upon the body in the first formula, while describing the whole unit of space that is expended upon the body in the second formula, while describing the first 1-64th, or the last 15-64ths of the whole unity of space; that is, in either formula, the amount of space described at any given instant of time during the fall over the given space, will be the square of the amount of force then and there expended from the commencement of the fall; and in such case passive time will have effected nothing, or will have had no effective or physical agency in the matter, any more than space or distance has; it will only have awaited the operation of force according to the rate or intensity of the force applied, precisely as in the case of the mechanical powers, in which case the whole time reciprocates with the rate or intensity of the force employed, 
But 1 will defer for the present a further investigation of the law of gravity in and through a consideration of the law of falling bodies, as it is my intention to refer to and examine this very important subject again; and until I can induce a consideration of the subject by others, I wish to present the subject in various ways, (and they may be multiplied to any extent,) with the view to ascertain whether that great, eternal and universal law, upon which we all suppose we have a right to calculate, (viz. that the whole amount of time employed or rather passed over during a mechanical operation, is the simple inverse or reciprocal of the rate of force employed) has anywhere, or in any case, been abrogated, repealed or altered ; and whether time, instead of passively awaiting an operation, has become the principal operator. For if such be the case, it is certainly as important to philosophy that it be known, as Mr. Rees or any other person can consider it. But surely such could not have been suspected previous to its discovery, or its being found by Newton, after a search of some twenty years, (according to his biographer,) and even then, under such ecstatic agony as compelled him to ask a friend to pick up and secure what had been found; and the importance of which discovery was such as to require two years in penetrating its consequences.

In respect to the line of direction, which a body falling towards the earth will form or describe, - which was considered and mooted between Newton and Mr. Hook, as though the same were to be settled by convention, - Hook suggested, that if the body fall in a vacuum, the line would be an eccentric ellipse; and also suggested such eccentric ellipse as the consequence of a force inversely proportional to the squares of the distances from the earth's centre. In relation to this matter, it is said that Newton, "having examined this suggestion by mathematical calculations, found that an attractive force, emanating from a centre, and acting inversely as the squares of the distances, would produce motions exactly resembling planetary motions, both in regard to the form of the orbit and the velocity of the body at each point ;"' and this, he says, " was the secret of the system of the world; but it still remained to account for the discordance of the moon's motion, which had before (1665) embarrassed Newton." Now what Sir Isaac Newton is said to have found by such mathematical calculations, has been quite liberally endorsed to the world as a demonstrated truth. But I predict that the world (sooner or later) will esteem it as great an imposition as could well have been palmed off upon it. Thus Mr. Rees, in order to help Newton's moon story out of the fog in which it is enveloped, says, that the centripetal force of the moon is known from 
the elliptical figure of the moon's orbit to be reciprocally proportional to the square of the distance.

Nevertheless, even after this supposed mathematical discovery in respect to Hook's suggestion, the motions of the moon were still discordant until they were reconciled to the same mathematical disclosure by a deduction of the Newtonian law of gravity, from a consideration of the law of falling bodies, which was almost impenetrable in its consequences, and laid the foundation for the immortal "Principia."

We see, then, what supposed results are attempted to be linked together, or placed in the same category, however discordant, to induce a belief that the force of gravity varies inversely as the square of the distance varies; and that the same has been so found or demonstrated by Sir Isaac Newton, - the most conclusive method, and that of which the modus operandi is given, (finished out by a friend of Newton,) being the one arising from a consideration of the law of falling bodies.

The law of falling bodies, then, from which the law of gravity may be easily deduced; and in which no error or mistake in the course of the investigation need to occur; (unless it be in assigning some active operation to time,) ought, again and again, to be investigated. I am aware that time has been so far personified, as to make it the principal actor in our world, and even in the universe. We speak of the hand, the tooth, the foot, the track of time; of its ravages, of the work of time, \&c.; we speak of beguiling time, and even of killing time. Such personification, however, is not poetic enough to give us a clear idea of the species of animated nature to which time belongs; but when we find time seated at the festive board, with ermine beard and forelock gray, having laid down his scythe and glass, and become a cheerful spectator of the proceedings, we can have but little doubt of its human nature, even though it should not otherwise partake of the festivities.

Nevertheless, after all this personification of time, in matters of philosophy, we ought not to assign to time a task, a labor, or an operation which it is wholly incompetent to perform.

\section{SECTION FOURTH.}

In an investigation of the law of gravity by the use of the means which have usually been resorted to by those who have attempted the investigation, viz. a consideration of the law of falling bodies, it may not be amiss, as preliminary thereto, to quote from those authorities which may be supposed to contain the best expositions of the Newtonian method of investigation. 
Colin Maclaurin, who was a celebrated mathematician, a contemporary of Sir Isaac Newton, and an admirer of his philosophy and discoveries; and who wrote an account of them in four books, which passed through several editions, thus gives us the Newtonian exposition or deduction of the law of gravity from a consideration of the law of falling bodies. "The computation may be made in this manner, - the mean distance of the moon from the earth being sixty times the distance of heavy bodies at the surface from its centre, (a body at the surface will fall 15 $\frac{1}{12}$ Parisian feet in one second, ) and her gravity increasing in proportion as the square of the distance from the centre of the earth decreases, her gravity would be $60 \times 60$ times greater near the surface of the earth than at her mean distance, and therefore would carry her through $60 \times 60 \times 15^{\frac{1}{12}}$ Parisian feet in a minute, near the surface; but the same power would carry her through $60 \times 60$ times less space in a second, than in a minute, by what has been often observed of the descent of falling bodies; and therefore the moon, in a second of time, would fall by her gravity near the surface of the earth $15 \frac{1}{12}$ Parisian feet, which therefore is the same with the gravity of terrestrial bodies." I will also refer to Rees' Cyclopædia, Vol. XXV, under the article Moon, in which the subject under consideration is treated of; when, after a comparison of the sun, moon, and earth, as to density, proportional magnitude, \&c., and after some remarks on the motion of the moon's apogee, its irregularities, \&c., we find the following observation, viz., "The irregularities above enumerated, and some others of a similar kind, have been urged as objections to the Newtonian theory of gravity, though they were anticipated by the illustrious author, who not only evinced their consistency with it, but suggested the explication of them which might be deduced from that theory properly understood and applied." Mr. Rees then quotes from Mr. Vince, commencing with the familiar expression, "Sir Isaac Newton having found," \&c., and finally observes, that "Mr. Euler also retracted his own erroneous opinion in deference to the judgment of Mr. Clairaut, and concurred with him in doing ample justice to the Newtonian theory," \&c. Mr. Rees proceeds to say, that "It would not be consistent with the limits or nature of this work, [the Cyclopædia,] to investigate, by tedious and elaborate processes of an analytical and geometrical kind, the various equations that have been explored for the illustration of these laws," and refers the reader to Vince's Complete System of Ástronomy, Vol. II. Chap. XXXII. Mr. Rees then proceeds: "We shall, however, in this place, introduce a general view of the Newtonian theory of gravity, as it is applied to the solution of the irregularities of 27. 
the moon's motions." "We have already, under the article Gravitation, illustrated and confirmed the Newtonian theory of gravity, as it regards the moon and other planets; but as the subject is of importance, and as it is immediately connected with what follows, we shall here give a concise statement of the leading facts by which the identity of the centripetal force as it respects the moon and that of gravity, was originally explained and established, referring, for a more detailed account, to the article just cited."

"It is well known and universally allowed, that the planets are retained in their orbits by some power which is constantly acting upon them; that this power is directed towards the centres of their orbits; that the efficacy of this power increases upon an approach to the centre, and diminishes by its recess from the same; and that it increases according to a certain law, namely, that of the square of the distance as the distance diminishes; and it diminishes in the same ratio as the distance increases. Now by comparing this centripetal force of the planets with the force of gravity on the earth, they will be found perfectly alike. This we shall illustrate in case of the moon, the nearest to us of all the planets." "The rectilinear spaces described in any given time by a falling body, urged by any powers, reckoning from the beginning of its descent, are proportionable to those powers. Consequently the centripetal force of the moon, revolving in its orbit, is to the force of gravity on the surface of the earth, as the space which the moon would describe in falling any little time, by her centripetal force towards the earth, if she had no circular motion at all, - is to the space which a body near the earth would describe in falling by its gravity towards the same." "By a very easy and obvious calculation of these two spaces, it will appear that the first of them is to the second, - that is, the centripetal force of the moon revolving in her orbit is to the force of gravity on the surface of the earth, - as the square of the earth's semi-diameter is to the square of the semi-diameter of her orbit, which is the same ratio as the moon's centripetal force in her orbit is to the same force near the surface of the earth. The moon's centripetal force is therefore equal to the force of gravity. These forces consequently are not different, but they are one and the same; for if they were different, bodies acted on by the two powers conjointly would fall towards the earth with a velocity double that arising from the sole power of gravity. It is evident, therefore, that the moon's centripetal force, by which she is retained in her orbit and prevented from running off in a tangent, is the very power of gravity of the earth extended thither." Mr. Rees then proceeds to give the method of investigation after the 
manner of Maclaurin, and also of Mr. Vince, namely, that Sir Isaac Newton found that the moon is drawn by its centripetal force from a tangent to its orbit towards the centre of the earth, about $15 \frac{1}{12}$ Parisian feet in one minute of time; and that a body will fall from a state of rest near the surface of the earth 15 1 Parisian feet in a second of time; and that the distance of the moon from the centre of the earth is about sixty times as great as the surface of the earth is from its centre, \&c. Mr. Rees then says: "But this force (centripetal force of the moon) being known from the elliptical figure of her orbit to be reciprocally proportioned to the square of the distance, would impel the moon, supposed to be at the surface of the earth, through a space equal to $60 \times 60 \times 15_{\frac{1}{12}}$ feet in one minute." "But bodies impelled by the force of gravity fall, near the surface of the earth, through the space of $15 \frac{1}{12}$ Paris feet in one second, and the spaces being as the squares of the times, through $60 \times 60 \times 15_{1}^{\frac{1}{2}}$ in a minute. Consequently, as the force by which the moon is retained in its orbit, and the force of gravity, produce the same effects in the same circumstances, and tend towards the same point, they are the same force." Mr. Vince, who seems to have been taught according to the perfect manner of the law of the fathers, and who appears to be a very honest recorder of the philosophy extant, from some cause seems to have avoided so detailed an exposition of the Newtonian method of deducing the law of gravity, as some others have presented; and in place of it find the following, namely: "Sir Isaac Newton found that if we the force with which bodies fall upon the earth's surface were extended to the moon, and were to vary inversely as the square of the distance from the centre of the earth, it would in one minute draw the moon through a space which is equal to the versed sine of the arc which the moon describes in one minute. He concluded, therefore, that the moon was retained in its orbit by the same force as that by which bodies are attracted upon the earth."

I have thought proper to quote thus liberally from those text books which have been received as authority, (upon a subject which Mr. Rees considers important,) from the time of the dispensation of the law by Sir Isaac Newton, until it became wholly unnecessary to do more than simply to declare the law, namely, "that the force of gravity varies inversely as the square of the distance varies."

The subject is not less important than it is considered to be by Mr. Rees, and consequently is entitled to as much consideration as will serve to disclose a truth so important to be known. The subject is not a new one, requiring only a simple disclosure 
and development to the world, but as it has been husbanded and fortified about by eminent tacticians and engineers, until at length the world have assented that the premises are legitimately and rightfully possessed, (especially after the adhesion to them of such men as Clairaut and Euler,) something more must be done than simply to disclose the truth to unprejudiced minds. Error must be broken up and dispersed; a breach and havoc must be made in the citadel; the old standard must be torn down and a new one firmly placed in its stead, with legible inscriptions thereon which all may read; and should such standard appear more republican or less regal than the old, it may only indicate one step in the march of modern improvement. Hence it may not be inappropriate, as preliminary to a more direct investigation of the subject, that I should remark or comment somewhat upon the text just quoted from Maclaurin, Rees and Vince; and also upon other extracts which may be taken or considered in connection. Saying nothing about the discrepancies which may appear in these quotations when taken in connection, I shall take the liberty to discard from the investigation or comments, that barren, absurd and futile allegation which inexplicability required as a make-weight, and which in process of time was so opportunely added; and shall leave the same to be inquired of by the world at their leisure, - I refer to that allegation of Mr. Rees, (which, to be sure, seems rather in the nature of an exclusion of a conclusion,) that " this force [the centripetal force of the moon] is known, from the elliptic figure of her orbit, to be reciprocally proportional to the square of the distance," \&c., which naked allegation has been adopted as law, as in case of Norton's Astronomy, "designed for use as a text-book in colleges and academies;" in which, under the head of the "theory of universal gravitation," he says, "It is also proved by the principles of mechanics, that if a body; continually urged by a force directed to some point, describe an ellipse of which that point is a focus, the force by which it is urged must vary inversely as the square of the distance." Now allegations like this may be necessary for the establishing of the Newtonian law of gravity, if found to be inexplicable without them; but they are not necessary for the furtherance of philosophy, or the science of astronomy. If the orbits are elliptic, and if in an elliptic orbit the centripetal force is necessarily inversely as the square of the distance, and if this were proved to be the case, then, to be sure, Rees' allegation would be well enough, because true; but as it is, the very fact is assumed, a priori, for which the inquiry was being made. An ellipse may vary from the circle by any given rate of ellipticity ; consequently the variation may be less than any assignable 
quantity; and consequently we all agree, that if the force of gravity be inversely as the distance in the ellipse, it will also be so in the circle; and, $e$ converso, if the force be inversely as the distance in the circle, it will be also in the ellipse, if a planet shall be found revolving in such orbit. But the simple laws and principles of dynamics, so readily evolved and explained, are driven or warped from their legitimate sphere, for the purpose of establishing belief in a hypothesis which yet requires investigation to determine its truth or error. It cannot be necessary that heaven and earth should fail, rather than that Sir Isaac Newton should be found to have been mistaken, either in his theory or law of gravity.

As a prelude to the commentaries intended upon the quotations before made, and also to such further matter as. I may put forth for consideration, perhaps no better can be offered than an extract from Colin Maclaurin, (the friend and admirer of Sir Isaac Newton and his philosophy,) in which he sets forth the tribulations of science in most appropriate remarks, as in the following:

"An entire liberty must be allowed to our inquiries, that natural philosophy may become subservient to the most valuable purposes, and acquire all the certainty and perfection of which it is capable ; but we ought not to abuse this liberty by supposing instead of inquiring, and by imagining systems instead of learning from observation and experience the true construction of things. Speculative men, by the force of genius, may invent systems that perhaps will be greatly admired for a time. These, however, are phantoms which the force of truth will sooner or later dispel, and while we are pleased with the deceit, true philosophy, with all the arts and improvements that depend upon it, suffers."

"The real state of things escapes our observation, or if it presents itself to us, we are apt either to neglect it wholly as fiction, or, by new efforts of a vain ingenuity, to interweave it with our own conceits, and labor to make it tally with our favorite schemes. Thus by blending together parts so ill suited, the whole comes forth an absurd composition of truth and error." "Of the many difficulties that have stood in the way of philosophy, this vanity perhaps has had the worst effects," \&c. " "All other causes have not done so much harm as that pride and ambition which have led philosophers to think it beneath them to offer anything less to the world than a complete and finished system of nature; and in order to obtain this at once, to take the liberty of inventing certain principles and hypotheses, from which they pretended to explain all her mysteries." Mr. Maclaurin, however, in speaking of Sir Isaac Newton's philosophy, evinces no 
want of confidence in its perfection; for he says that Sir Isaac Newton " has secured his philosophy against any hazard of being disproved or weakened by future discoveries." Mr. Maclaurin, however, laments bitterly, approximating even to poetic agony, that the Newtonian philosophy should have been so long resisted and delayed. He says, "It was, however, no new thing that this philosophy should meet with opposition, \&c., and that it should struggle 'with the prejudices of those who had accustomed themselves to think only in a certain systematic way; who could not be prevailed upon to abandon their favorite schemes, while they were able to imagine the least pretext for continuing the dispute; every art and talent were displayed to support their falling cause; no aid seemed foreign to them that could in any manner annoy their adversary; and such often was their obstinacy, that truth was able to make but little progress, till they were succeeded by younger persons, who had not so strongly imbibed their prejudices.".

Mr. Maclaurin having fully imbibed the Newtonian theory and law of gravity, and also his projectile hypothesis, namely, that the planets must of necessity have originally been projected in tangents to their orbits, with forces so proportioned to their powers of innate or inherent gravity, as to enable such innate gravity to form the orbit, either more or less eccentric, according to the original force of projection, (which he calls "the velocity of projection,") - then labors to show how a planet will proceed in its orbit, - what kind of curve it will form when projected in a tangent to its orbit, with a greater or less force, at a greater or less distance from the sun, - what kind of an ellipse it would form with a greater or less projection, which philosophy he has embellished and elucidated by a multiplicity of diagrams, which

"Gird the sphere with centric and eccentric, scribbled o'er"

with all the fanciful notions in regard to the manner in which bodies would proceed, - what curves they would form, - how the force and the motion would alternately get the better of each other, - how a body might forever continue to recede from the sun; or upon the other hand, how it might directly or eventually fall into the sun, \&c., were the laws of nature which govern the universe, altogether different from what they are; - together with a vast amount of similar matter, well calculated to bewilder the mind into the belief that the force of gravity is inversely as the square of the distance, - that the planets were necessarily projected in tangents to their orbits ; and hence, - that a planet may revolve in an eccentric orbit, and still obey the Newtonian law of gravity, - it is necessary to attach a kind of balance-wheel, 
called the centrifugal force, to enable the force and motion alternately to get the better of each other at the proper times and places. And in respect to the centrifugal force, I will here give a quotation from the author. "The gravity indeed at the lower apside, is greater than the gravity at the higher apside, in proportion as the square of the distance is less. But the centrifugal force arising from the circular motion about the sun, increases in a higher proportion, namely, as the cubes of the distances decrease, for these centrifugal forces are in the direct proportion to the squares of the velocities, and their inversed proportion of the distances compounded together; the first of these in the inverse proportion of the squares of the distances, and the two together compound the inverse proportion of the cubes of the distances. The centrifugal force therefore increases in a more rapid ratio than the force of gravity; and though the force of gravity prevails in the higher part of the orbit, the centrifugal force gets the better in the lower part of it. The force of gravity prevailing in the higher apsis, makes the planet approach the sun, the centrifugal force prevailing in the lower apsis makes the body recede from the sun; and the continued action of these two forces causes the planet forever to revolve from one to the other."

Hence, if we are to believe Mr. Maclaurin, the burden of the Newtonian philosophy in respect to the law of force and motion by which a planet revolves in its orbit, is, to use the very language or expression of Maclaurin, "that the force and motion alternately get the better of each other." This then is the only possible mode devised for compelling the planets to obey the Newtonian law of gravity, as promulgated ; for even Mr. Maclaurin, had there been "an entire liberty allowed to his inquiries," (which he so well recommended in the outset,) could have devised a much better law; for after all his labors, wanderings and perplexities, to which he was doomed in consequence of having implicitly imbibed the Newtonian law of gravity, we find him alleging and even repeating the allegation, that the proper limit of gravitation, by which the planets could best revolve, would be, "the inverse simple proportion of the distance," and he attempts to show how beautifully a planet would revolve from one apsis to the other, if operated upon by a force of gravity, decreasing in the reciprocal simple proportion of the distance.

I have thus far quoted from, and referred to the works of Mr. Maclaurin, with a view to show what the obstinate world had to contend with, inasmuch as he was contemporary with Sir Isaac Newton, but long survived him; and as he became the able expousder of the Newtonian philosophy to the world, and especially of the modus operandi by which the laws of planetary 
force and motion were to operate in accordance with the Newtonian theory and law of gravity, it might naturally be supposed that throu $\_$h Maclaurin, we should drink from the highest and purest streams of the Newtonian philosophy. But the inquirer had better examine the work if it be accessible.

Gravity may be technically defined by saying that it is the force which urges a planet towards the sun, and this force is called the gravity of the planet, or its force of gravity towards the sun; and in case of a body falling towards the earth from a state of rest, the motion of which is uniformly accelerated from the beginning or commencement of the fall, - there being no space however small, without acceleration, - the force which constantly accelerates the motion may be called either the accelerating force, the soliciting force, or the attractive force, or force of gravity.

Now the specifio manner in which the computation is made, whereby the Newtonian law of gravity is supposed to have been deduced from a consideration of the law of falling bodies, as reported by $\mathrm{Mr}$. Maclaurin and by Mr. Rees, is substantially the same, and therefore requires the same comment; nevertheless Mr. Rees has liberalized somewhat in his preparation, which may also require a passing remark; but from some peculiar ambiguities in the version given by Mr. Vince the same may require a somewhat different consideration. By Mr. Maclaurin and Mr. Rees, the unit of distance from the centre of gravity is placed at the surface of the earth, or the semi-diameter of the earth from the centre of gravity; while the moon is at the distance 60 , or 60 semi-diameters of the earth from its centre. The unit space to be passed over at the unit distance is $15_{\frac{1}{12}}^{\frac{1}{2}}$ Parisian feet, and is properly denoted by unity or 1 , whether it be one rod, or more or less than one rod. And the unit of time in which the unit of space is to be described at the unit distance, is one second of time. At the distance 60 the unit of space will be described in 60 units of time.

And from these premises it is required to ascertain and assign the proportion or rate of gravity at the distance 60 , to that at the distance 1. Hence the rate of gravity or the force of gravity at the unit distance, or distance 1 , must also be assumed at unity; for force is also quantity, and may as well have its quantity numerically assumed as any other quantity, - as that of distance, space or time. Nor is there any other rational or proper way for obtaining the proportion, rate or intensity of gravity at the distance of 60 , to that at the distance 1 , than that of assuming the rate at the distance 1 , at unity, - by which that at the distance 60 is to be compared, - as will be readily seen by any one on a little 
consideration of the economy of numbers, their use and perfect adaptation in the expression of quantity of whatever denomination, and more especially in that transcendental property of ratio and proportion, which is only developed by the powers and roots of numbers, (the centre of all ratio and proportion being in unity;) and hence all quantities which may be numerically expressed, whether of the same or of different denominations, may be proportioned to each other and to unity, that is, may have their ratios numerically assigned. The proper elements, then, for determining the law of gravity from the law of falling bodies, are, - distance from the centre of gravity, time, and space; namely, the time in which a given operation is to be performed, and the space to be described or passed over in a given time; and force, or the force of gravity, which is the agent, the cause, the physical power, which is to perform the labor or operation; which is to urge the body over a given space in a given time. Force then is the active or efficient agent or element, in the operation; while the time and space passed over, (I will not say the time employed, or the time spent,) are as passive as is the distance. And if we have so far and so long been in the habit of personifying time as being a physical agent or operator, that we cannot divest ourselves of that form of thought, we had better avoid the error by supposing or imagining that time waits for the operation to be performed, or for the labor to be accomplished, that is the labor of urging a ponderous body over a given space; and by such consideration we shall find ourselves sustained, corroborated and supported, by every principle of mechanics or dynamics with which we are acquainted, or which has ever been disclosed. The rate, intensity or efficiency of the force employed or required in raising a ponderous body over a given space is inversely or reciprocally as the whole time passed over during the operation. Every provident man pays his hireling for the efficient labor performed, rather than for the time passed over. Nevertheless, if the Newtonian law of gravity is true, no proposition can be plainer or more easily comprehended, than that fifty-nine sixtieths of the unit of space (namely, of the $15_{\frac{1}{1} 2}^{\frac{1}{2}}$ Parisian feet) passed over or described a the distance of the moon, or distance 60 , is wholly the operation or the effect of time; that is, but one sixtieth part of the physical or effective force will be expended on the body at the distance 60 , in urging it over the unit of space that will be expended on the body at the distance of 1 , in urging it over the same amount of space, namely; the unit of space, or the $15_{\frac{1}{12}}$ Parisian feet.

I have said that distance, time, space, and the rate of gravity, were the proper elements from which to deduce the law of grav- 
ity, from the law of falling bodies ; and that the quantity of time, and space, and the rate of gravity, must be assumed at unity, for that body which is to commence its fall at the distance of unity; our object being to find the relative or proportional efficiency or intensity of the physical agent or operator that is to perform the operation in proportion to the distance. For, although the proportions of the rates of velocity of the two bodies (conceiving them to commerice their fall at the same instant of time,) are to be assigned, and consequently the rate of the velocity of the body commencing its fall at the distance of unity, at any given instant of time during the joint fall, or at any point of space equi-distant from the point from which they respectively commence their fall, is to be assumed at unity, that the rate of velocity of the other may be assigned or proportioned to unity; nevertheless, this proportion of velocity at any given instant of time, and consequently at any point of equal space described by both bodies from the commencement, is a deduction which readily follows and discloses itself from a proper consideration of the other elements, - namely, distance, time, space, and the rate of gravity. Nor has there been error in properly assigning this rate of velocity, (calling that unity at the distance of unity,) for all agree that the body at the distance 1 , starts with a velocity 3600 times as great as does the body at the distance 60 ; that when the body at the distance 1 , has passed over half a second of time, its velocity will be 3600 times as great, as that at the distance 60 , when the body has passed over half a second of time; that when the body at the distance 1 has passed over one foot of space; its velocity will be 3600 times as great as that at the distance 60, when the body has passed over one foot of space, \&c. We then speak properly, when we say the rate of the velocity is inversely as the square of the distance. But we must never forget, in our investigations, - what all will allow to be an axiom as sound as any which has been adopted as such, - that the velocity of a falling body is accelerated from the beginning of the fall ; that is, there is no part of the space passed over, however small, in which the motion is not accelerated; and for this reason, the determination will become absolute and conclusive, that the rate or intensity of gravity, varies inversely as the distance varies. But these principles will receive farther consideration and elucidation in the proper place.

Now I will not hesitate to declare that a more bungling, erroneous and hap-hazard exposition of the law of gravity, could scarcely have been devised by the ingenuity of man, than that put forth by Maclaurin, which has so long stood the test of time, for surely no mental or physical operation has affected it. And 
on reading it, one might be well supposed to exclaim, Is it no matter, - of no consequence, upon what premises the fabric of astronomy is reared, or in what quagmire its foundations are laid? And is it possible that such an exposition could have thrown mankind into those inexplicable difficulties under which they have been laboring for the last two centuries?

Mr. Maclaurin, after setting forth in his premises his unity of distance, namely, the surface of the earth; his unity of time, viz., one second; his unity of space, viz., $15_{\frac{1}{12}}^{\frac{1}{2}}$ Parisian feet; and the distance of the moon, viz., 60 units of distance; proceeds thus: "And her (the moon's) gravity increasing in proportion as the square of the distance from the centre of the earth decreases, her gravity would be $60 \times 60(3600)$ times greater near the surface of the earth than at her mean distance, and therefore would carry her through $60 \times 60 \times 15_{1 \frac{1}{2}}^{1}$ Parisian feet, $(3600$ units of space,) in a minute, near the surface."

Now what the author actually meant by this passage must perhaps rest somewhat upon conjecture. "Her gravity increasing inversely as the square of the distance decreases, her gravity would be 3600 times greater near the earth than at her mean distance." If he intended to convey the idea that the ponderosity of the moon would be 3600 times greater at the surface of the earth than at the distance of the moon, he is entirely welcome to the conjecture, and to the consequences that would follow, were the conjecture true in point of fact. The author surely could not intend that a body commencing its fall at the distance 60 , would, when it arrived at the distance 1 , have the same velocity that a body would then have that commenced its fall at the distance 1; for it is presumed that he defines gravity after the manner of other authors ; viz., that it is the force which urges the body towards the centre of gravity, and not the motion or velocity produced by gravity. If by any possible construction we could suppose that the author would only infer that if two bodies commenced their fall at the same time, one at the surface of the earth, and the other at the distance of the moon, that at any given instant of the fall, the velocity of that at the surface of the earth would be 3600 times greater than that at the distance of the moon, it would be strictly true in point of fact, but his premises assumed do not permit us to assign such as his meaning or intention ; for he says, "her gravity, increasing in proportion as the square of the distance from the centre of the earth decreases, would be 3600 times greater near the surface of the earth than at her mean distance."

And it is only upon the principle that the rate of gravity varies inversely as the distance, (not inversely as the square of the dis- 
tance,) that the velocity of a body at the distance 1 , is 3600 times greater than that at the distance 60 , at any given instant of their fall, both commencing at the same time. But why should that be assumed as a fact for the truth of which we are seeking, and the truth of which must necessarily be the result of the evidence from which it is deduced? Why commence by saying that the gravity of the moon increases in proportion as the square of her distance decreases, before the fact is ascertained, inasmuch as the rate of gravity, as proportioned to distance, is the very thing we wish to know? But the premises and conclusion intended to exhibit cause and effect, preceding the following words, viz., "and therefore would carry her through 60 $\dot{\times} 60 \times 15_{\text {I } 2}^{\prime}$ Parisian feet, (3600 unit spaces, $)$ in a minute, near the surface of the earth," would be equally vague, disjoined and destitute of meaning, even though it were a fact that the rate of gravity increases from the moon to the earth in the same proportion as the square of the distance decreases; there being no premises, conclusion or result whatever contained in the sentence, - nothing except absurdity. And it appears that this part of the exposition, was in time, suspected of discrepancy; nevertheless, the law must not fail; and hence we find, in the version given by Mr. Rees, that remarkable passage which I have already quoted and given over to the world for investigation and comment, viz., "but this force (meaning the centripetal force that retains the moon in her orbit) being known, from the elliptic figure of her orbit, to be reciprocally proportional to the square of the distance, would impel the moon, supposed to be at the surface of the earth, through a space equal to $60 \times 60 \times$ $15_{\frac{1}{2}}$ Paris feet (3600 unit spaces) in one minute." -This version only assumes a previous knowledge of the fact that the rate of gravity is inversely as the square of the distance; viz., "it being known from the elliptic figure of the moon's orbit."

Now we know that by the law of falling bodies, a body falling from a state of rest at the surface of the earth, will fall 3600 times as far in one minute, as it will in the first second of time of that minute; and hence, if a body were brought from the moon and were to commence its fall near the surface of the earth, it would doubtless be governed by the law of falling bodies. But I am wholly unable to discover that the expositors have in any way or manner connected this fact with that of the Newtonian law of gravity, viz., that the rate of gravity varies inversely as the square of the distance varies.

The result then of Mr. Maclaurin's reasoning seems to be this: that a body falling from a state of rest near the surface of the earth will fall $15_{\frac{1}{2}}^{\frac{1}{2}}$ Parisian feet in a second of time; that if 
the moon were to fall from a state of rest near the surface of the earth, it would fall 3600 times as far in one minute, as it would in one second, and that it will describe 3600 times less space in one second than in one minute, and therefore, the moon, in a second of time, would fall by her gravity, when near the surface of the earth, $15 \frac{1}{12}$ Parisian feet; which therefore is the fact with regard to the gravity of terrestrial bodies.

Thus far I have thought proper to comment on Mr. Maclaurin's exposition of the law of gravity ; wishing from the importance of the subject, (as Mr. Rees justly remarks,) to present the same in as bold relief as possible, with a view to solicit an investigation of the subject. And whether he "was possessed of many whims and conceits," as he alleged Kepler to have been, when comparing him with the illustrious Newton, must, perhaps, be inferred from his works.

Nevertheless, I think he has presented the world with a very strange code of laws, and a strange body of philosophy; yet no one doubts its being strictly Newtonian. It is not consistent with the limits of Mr. Rees' Cyclopædia to investigate the various equations that have been explored for the illustration of these laws; and in fact, to go into an elaborate process with a view to present such as were adopted by Clairaut, and by mathematicians in general, since Clairaut and Euler professed their belief in the Newtonian laws, would be an onerous task indeed; for already has the moon been subject to between thirty and forty equations for the illustration of these laws. Mr. Rees alleges, in his preliminary to the final exposition of the Newtonian law of gravity according to Maclaurin, that if is well known and universally allowed (among other things) that the efficacy of the force of gravity increases or diminishes in the inverse ratio of the square of the distance; upon which allegation I am bound to join issue. Mr. Rees also makes the following allegation, which is similar in its consequences to the foregoing, and consequently in accordance with the Newtonian law of gravity, and with Mr. Vince's information of what Sir Isaac Newton found, viz.: that the moon is drawn by its centripetal force from a tangent to its orbit towards the centre of the earth about $15 \frac{1}{12}$ Parisian feet, in one minute of time; or, as expressed by Mr. Vince in another place, "Sir Isaac Newton found that if the force with which bodies fall upon the earth's surface were extended to the moon, and to vary inversely as the square of the distance from the centre of the earth, it would in one minute draw the moon through a space which is equal to the versed sine of the arc which the moon describes in one minute. He concluded, therefore, that the moon was retained in its orbit by 
the same force as that by which bodies are attracted upon the earth." Mr. Rees alleges that "the centripetal force of the moon revolving in its orbit, is to the force of gravity on the surface of the earth, as the space which the moon would describe in falling any little time, by her centripetal force towards the earth, if she had no circular motion at all, is to the space which a body near the earth would describe in falling by its gravity towards the same;" - which sentence perhaps should be translated thus: "The centripetal force of the moon is to the force of gravity on the surface of the earth, as the space described by the moon in a rectilinear fall towards the earth, in some little given time, caused by the moon's centripetal force, is to the space described in an equal time by a body falling from a state of rest near the surface "of the earth ;" and such translation appears to be in exact accordance with what Mr. Vince alleges that Sir Isaac Newton found. And in corroboration of the version I have given, $\mathrm{Mr}$. Rees proceeds to say, that "the space at the moon is to the space at the surface of the earth, i. e.- the centripetal force of the moon is to the force of gravity on the surface of the earth, - as the square of the earth's semi-diameter, (square of the distance 1,) is to the square of the semi-diameter of the moon's 'orbit, (square of distance 60,) which is the same ratio as that of the moon's centripetal force to the same near the surface of the earth." And hence it is concluded, and that too from such premises, that the force of gravity on the surface of the earth, and the centripetal force of the moon, are identical ; the intensity varying in the inverse ratio of the square of the distance as we recede from the centre of the earth.

If then it has been ascertained that the centripetal force acting upon the moon would (if the moon had no circular motion in its orbit) draw the moon from a state of rest by an accelerated motion towards the centre of the earth as far in 60 seconds of time, as a body near the surface of the earth will fall from a state of rest in one second of time, we will consent that it furnishes indubitable proof of the identity of the centrepetal force of the moon with that of the force of gravity at the surface of the earth; the intensity or efficiency having its proportion assigned in the powers and roots of numbers in some given inverse ratio to that of the distance from the centre of the earth. But that such fact has not been ascertained or disclosed by the methods, or in the manner alleged by Mr. Maclaurin, Mr. Rees and Mr. Vince, will, perhaps, yet become too manifest for contradiction. For the very allegation which is calculated to sustain the reasoning upon the subject, namely: that gravity, or the intensity of gravity varies inversely as the square of the distance varies,throws the whole subject into inexplicable confusion, admitting 
of no rational result or conclusion. Nevertheless, "all nature cries aloud, through all her works," that gravity at the surface of the earth which causes bodies to fall, and the centripetal force of the moon which retains it in its orbit, are identical, or one and the same force, varying in its intensity in some given proportion to the inverse of the distance, which ratio is assignable in the powers and roots of numbers. Such was the opinion of Kepler long before the days of Newton, when he declared that the moon gravitated towards the earth, and that but for the moon's motion in its orbit, the earth and moon would come together, and that too, at a point as much nearer the earth than the moon, as the earth is greater than the moon. Such, too, was the opinion of Kepler when he disclosed and promulgated those eternal laws, "that the squares of the periods of the planets are as the cubes of their mean distances from the sun; and that the motion of any given or individual planet varies in its orbit inversely as its distance from the sun varies." And this, too, may be indubitably proved by a vast amount of evidence.

But the "a proof," which Mr. Vince says Sir Isaac Newton gave Dr. Halley, to me is no proof at all ; it being a proof in which the laws of Kepler were wholly thrown aside and disregarded, and in which the law of falling bodies was wholly misunderstood and perverted. And as to what Mr. Vince says that Sir Isaac Newton found, namely, "that if the force with which bodies fall upon the earth's surface were extended to the moon, and were to vary inversely as the square of the distance from the centre of the earth, it would in one minute draw the moon through a space which is equal to the versed sine of the arc which the moon describes in one minute." I shall, in some future section, - in which the element of deflection, (namely, the deflection of a planet from a tangent to its orbit,) which has been adopted and so consequentially considered in reference to central forces, will be somewhat liberally treated of, - endeavor to show that Sir Isaac Newton found no such thing; for the reason that no such thing existed in nature.

- But I have already prolonged this section, perhaps quite too far, in attempting to unravel the flimsy woof in which the law of gravity has been enveloped, or in cutting the meshes in which it has been entangled, inasmuch as the shreds and remnants, however carefully saved, can be of no future use.

But what has Mr. Rees, or any other commentator on the law of gravity, done with the point to be mooted? Where are their reasons, their evidence, their demonstrations, that show what the law of gravity is? Is it contained in a subsequent allegation of Mr. Rees, " that this force is known from the elliptical figure of the 
moon's orbit, to be reciprocally proportional to the square of the distance ?" Or is it contained in a preceding allegation, which is so boldly appended to certain principles which are well known and universally allowed, - that is, is it proved by his allegation, " that gravity increases as the square of the distance diminishes, and that it diminishes in the same ratio as the distance increases ?" Or shall we look for the proof in the allegation of Mr. Vince and others, that "Sir Isaac Newton found," \&c.? And if we do not find the evidence so fully developed in these allegations as to make it highly dangerous to doubt, I will say, let us reason together upon the subject, and endeavor to comprehend and understand the numerical developments of the great physical law of the universe. For, that the intensity of gravity. is proportioned to the distance from the centre of gravity, and that such proportion is inverse, or reciprocal in some given power or root of numbers, as that of the first or second, or some other, is not only universally allowed, but is a necessary consequence synthetically deduced from the great Keplerean laws, as well as from all phenomena arising from the motion of the heavenly bodies. What this proportion between distance and intensity of gravity is, Mr. Rees considers of importance ; at least, that it be established according to the Newtonian hypothesis; and hence he has thought proper to put forth the leading facts by which such hypothesis was originally explained and established. But if others should think it of equal importance that the law of gravity should be determined according to the truth, I have no fears as to the result.

Much stress, to be sure, has been placed upon the fact or circumstance, that $M$. Clairaut found out a very ingenious method of extricating himself from the evils of a long rebellion against the Newtonian law, by reconciling himself and the moon to its control ; and M. Euler escaped by retracting his opinion in deference to the judgment of $M$. Clairaut; and if this were conclusive evidence in respect to the law, surely we need not search farther, and may rest assured, that what Mr. Rees calls "the various equations which have been explored for the illustration of these laws," are in reality but the commencement of trouble.

I have already remarked upon Mr. Rees' premises and his supposed consequent. Nevertheless, as I also consider the subject to be of importance, it may not be wholly amiss to repeat the same and append a comment or two, by way of investigation. The truth he alleges is thus: "The rectilinear spaces described in any given time by a falling body urged by any powers, reckoning from the beginning of its descent, are proportionable to those powers." And his supposed consequent (and on this point the investigation is particularly required) is this : "Con- 
sequently the centripetal force of the moon, revolving in its orbit, is to the force of gravity on the surface of the earth, as the space which the moon would describe in falling, any little time, by her centripetal force towards the earth; if she had no circular motion at all, is to the space which a body near the earth would describe in falling by its gravity towards the same."

Now although the author has confused the expressions in which his supposed consequent is couched, beyond any of his predecessors, (for what reason I know not,) nevertheless, it may perhaps be rendered so as to obtain whatever of meaning it may contain, and at the same time be in accordance with the views of the illustrious author of the law of gravity, namely, that the centripetal force exerted on the moon will be to the force of gravity at the surface of the earth, as the fall of the moon from a tangent, in some little time, (say one second,) will be to the space described by a body falling from a state of rest at the surface of the earth in the same time. Such would surely be the case if the intensity of gravity were inversely as the square of the distance. But the question in respect to what proportion the intensity of gravity bears to the distance, is the one to be solved, and that too, through the medium of the phenomena which gravity produces. The law of gravity then is not to be assumed, a priori, but must await its development in and through its effects, or the phenomena which it causes, if so be it may thus be obtained. If Mr. Rees' deduction were true, it would, as he observes, be obvious that the centripetal force of the moon is to the force of gravity on the surface of the earth as the square of the earth's semi-diameter is to the square of the semi-diameter of the moon's orbit; which would be the same ratio as that of the moon's centripetal force to the same force near the surface of the earth; and such centripetal force would in such case be sufficiently identified with that of gravity near the surface of the earth, as was fairly suggested by Kepler. But I think Mr. Rees' eonclusion of this part of his exposition, is quite too vague and full of unknown consequents to merit very serious. attention; and perhaps but little knowledge can be extracted from the same, except it be of the confusion of the subject under investigation. In treating of the identity of the centripetal force of the moon, and that of gravity near the earth, he says, "these two forces consequently are not different, but they are one and the same; for if they were different, bodies acted upon by the two forces conjointly, would fall towards the earth with a velocity double to that arising from the sole power of gravity. It is evident therefore, that the moon's centripetal force by which she is retained in her orbit and prevented from running off in tangents, is the 
very power of gravity of the earth extended thither." Now perhaps there are those who can discover some explanation or elucidation of the subject in the last quotation, and to such it must be referred; while it is my business to admire the unerring precision with which nothing but error has been produced.

Having in this work treated somewhat liberally of the law of falling bodies, more with a view of disclosing those errors which have resulted from attempts to elicit the law of gravity from a consideration of the phenomena incident to falling bodies, than to obtain therefrom the law of gravity, (believing that the law of gravity may be much more easily, simply, and satisfactorily deduced from a direct consideration of the Keplerean laws,) I shall not do more in this place than to offer, a few brief and desultory hints in respect to the law of falling bodies. I will use figure 1 to denote unity; what I will call one moment of time may be considered as being one second; the moon shall be considered at the distance 1 from the centre of the earth; and consequently, the surface of the earth will be at 1-60th of 1 from the centre of the earth, or centre of attraction; the amount of space described in one moment by a body falling from a state of rest at the distance of the moon, or distance 1 , will be called one space; hence the amount described in one moment by a body falling from a state of rest at the surface of the earth, or at the distance 1-60th of 1 , will be 3600 spaces, for the number of spaces described in the given time is necessarily reckoned in the sum of the odd numbers, commencing with the unit space described in the unit of time by the body whose distance is assumed at unity or 1 ; and hence, the number of spaces described in the unit of time is always (properly considered) the inverse of the square of the distance, (if so be that the greater distance is assumed at unity ;) that is, making the body whose distance is assumed at unity the standard, the proportional amount of space passed over in the given unit of time, will always be inversely as the square of the distance, (but not of the time,) and it is my purpose to show that it is always the square of the whole amount of force applied during the fall. In this example, then, the body at the distance 1 , or distance of the moon, is the standard; and the time of the operation is one moment, or one second; and in that time the amount of force expended on the body from the commencement of the fall is $1,-$ so, also, the rate or intensity of the force is 1 , the body at the surface of the earth is at the distance of 1-60th part of 1 , and the time of the operation is one moment or one second; and it is our business to ascertain the ratio or intensity of the force at the surface of the earth compared to that of unity, or that exerted at the distance 
of the moon; as also the proportional amount expended on the two bodies in the second or unit of time; that expended at the distance of the moon being 1 .

It having been well proved by Galileo and others, that the numerical law of falling bodies may be simply presented thus,

\section{$\begin{array}{llllll}1 & 1 & 1 & 1 & 1 & 1\end{array}$ \\ $\begin{array}{lllllll}1 & 3 & 5 & 7 & 9 & 11\end{array}$}

and so on, ad infinitum, from the commencement of the fall; the uniform figure 1 denoting an equal division of the time, and also of the amount of force expended in the fall, whether the intensity of the force be much or little; which time or force thus divided, may be called the equal times or the equal forces; and figure 1 in the series of odd numbers denoting the amount of space, or the space described by the body in the first time, (called the unit of time,) or while operated upon by the first force, or unit of space; each succeeding odd number denoting the number of unit spaces described in another unit of time or unit of force; and the sum of all the odd numbers being the square of the sum of all the units of time, or of all the units of force expended during the fall ; - I say, such having been determined or found to be the numerical law of falling bodies, perhaps but few propositions present themselves to the mind which can be more readily solved than the following, viz. the amount of force applied from the commencement of the fall during any given time, or while passing over any given space, accumulates with, or in the same ratio or proportion as the square root of the whole space then described. Hence the whole amount of force that has been applied at any given instant of the fall, or at any given point of space, is the square root of the whole amount of space described. And this principle in respect to force and space as applied to falling bodies, is invariable and universal; whether the intensity of the force be great or liftle; or whether the body commenced falling at the distance 1 , or at the distance 60 , or at the distance $1-60$ th from the centre of gravity. Thus, if the distance of the moon be assumed at unity, then the space described in a given moment of time, (however short,) called the unit of time, will be the unit of space also ; and acceleration will have been the consequence, even in this unit of space, however sinall; and a body commencing its fall at the surface of the earth or distance $1.60 \mathrm{th}$, will describe 3600 of those units of space in the same unit of time. Hence, at the surface of the earth the arnount of space described in the unit of time is inversely or reciprocally as the square of the distance; and this principle is universal, and holds good, whatever the distance may be which is assumed at unity. 
This inverse or reciprocal attitude or position of time and space, (the two passive elements observed in the phenomena and used in the investigation of the law of falling bodies,) is necessarily presented in this wise, viz. the unit of time being the same at the distance of the moon as at the surface of the earth, if we then assume the unit of space passed over in such unit of time to be at the distance of the moon, 3600 of them will be passed over in the unit of time at the surface of the earth. But if we assume that as the unit of space which is described by the body at the surface of the earth in the unit of time, then the amount of space described at the distance of the moon in the unit of time will be less than the unit of space at the surface of the earth, or 1-3600th part of the same. Thus the amount of space described in a given time or unit of time from the commencement of the fall is inversely or reciprocally as the square of the distance at which the body commences its fall. Let, then, the time be given (called one moment, or the unit of time, however long or short) in which the operation is to be performed. Let the space to be described in such moment (from the commencement of the fall) be one space; and when thus considered in the abstract, the original form of the mind will not permit us to consider either the rate or intensity of the force, or the whole amount of the force expended while describing such unit of space, to be other than unity, or more properly the square root of unity (for we are endeavoring to ascertain ratio or proportion as developed by the powers and roots of numbers.). Then suppose the distance at which the fall is to commence to be lessened to 1-60th part, and consequently that the number of units of spaces described in the given moment will be increased to 3600 , made up of the sum of the odd numbers in their order. Now perhaps no proposition can become more manifest in its solution than that the amount of force expended in the given moment, is just the square root of the amount of the space described; for the force being uniform, will of course, in order to correspond with the number of spaces, be divided into 60 parts ; the same amount being expended in describing the three spaces next succeeding the first, as is expended in the first; and consequently the amount of force expended in describing the first space is equal to that expended in describing an equal space or the unit space at 60 times the distance; and consequently, in either case, the amount of force expended is the square root of the amount of the space described; and hence the conclusion is inevitable, that the rate or intensity of the force is inversely as the distance. And now, aside from demonstration, whether such consideration of the subject be more or less, 
rational or satisfactory to the mind, than that which has so long received the popular sanction, remains to be determined, viz. whether physical force or whether passive time, which has no physical agency in the matter, shall have the credit of performing the operation, viz. that of urging a body of ponderous matter over a given space; for the fact will not be controverted for a moment by any one, that by the popular determination in respect to the law of falling bodies, time is a far more efficient agent than force; and that if one body commence falling at the surface of the earth, and another at the distance of the moon, when they shall have passed over equal spaces, 59-60th of the fall of the body at the distance of the moon will be credited to time alone, which, in fact, has only been passive, awaiting the operation, without having any physical agency whatever in the matter. Nevertheless, every provident person rather intends to give his hireling credit for the efficient force expended in the accomplishment of an operation, than for the time that may happen to be spent while the operation is being performed.

Thus have I, from a consideration of the importance of the subject, again referred to the law of falling bodies, and its consequent connection with the law of gravity, viz. the rate or intensity of gravity as proportioned to the distance from the centre of gravity; and I can well hope that the importunity, the urgency, and the anxiety which I have displayed on the subject, will not be set to my account as an attempt to disparage the judgment, the sagacity, or the perception of an enlightened world. Whatever "Sir Isaac Newton found," the world has been extremely loth to lose; and perhaps it might be rationally supposed, that, in a matter so simple, Sir Isaac could not have found amiss; and hence, that the subject could not require investigation.

\section{SECTION FIFTH.}

So far as philosophy depends upon numbers (whether numerically, mathematically or geometrically considered) for its development, it is in vain to attempt treating of subjects with any degree of unity of purpose, unless we assume unity, or 1, as a standard in all our investigations on which to rely for a development of the truth concerning the great law of ratio and proportion between quantities, whether of the same, or of different denominations ; for ratio properly flows from or to unity, as from or to ratio in unity; and in unity the roots and powers are equal. Hence if unity be always assumed as the standard in the comparison of allquantities susceptible of a rational compari- 
son by means of numbers, we thereby obtain our results with ease and facility, and in that unity of purpose by which they become manifest or conclusive to the mind. This I have endeavored to manifest in my investigations into the quadrature of the circle; as also when rising

\section{"Higher in the line \\ And variation of determined shape, \\ Where truth's eternal measures mark the bound Of circle, cube or sphere."}

And it is my purpose to show that unity is the proper standard in all our investigations in respect to the laws of force and motion as compared with those passive elements, - time and space; whether we consider the laws in reference to impulsive forces and the rate of motion produced thereby, - in which case it has been fully determined and shown that the rate of motion or velocity produced by an impulsive force is the root of the amount of force applied, - as that four times the impulsive force gives "twice the rate of velocity; sixteen times the impulsive force gives four times the rate of velocity, \&c. ; - or whether we consider the laws of force and motion in their most simple and common occurrence as produced by human or finite operations; viz. in directly overcoming the ponderosity of a body produced by the force of gravity in raising it through a given space by a uniform motion, (neither accelerated nor retarded,) by simple human force or power, unaided by any of the mechanical powers, properly so called, as that of the lever, or wheel and axle, \&c., in which case, each element proper to be considered is assumed at unity, or 1 , as that of the ponderosity of the body, - the given space passed over, - the time of the operation, - the rate or intensity of the force employed, - the rate of velocity with which the body moves, - as also the whole amount of force expended in the whole time of the operation; - or whether we consider the application of the mechanical powers in raising such body over a like space by a constant and uniform motion, but by the constant application of a less rate or intensity of force; in which case the time required will be inversely or reciprocally as the rate or intensity of the constant force applied; thus, if the rate of force be half of unity, the time required will be twice unity; if the time be 60 , the rate of force will be 1.60 th of unity, \&c. and the rate of velocity will be as the rate of force. Thus the elements of ponderosity, of space, and of the whole amount of force employed in the operation, will be as those in the foregoing example, viz. each will be assumed at unity, while those of time and the rate or intensity of the force employed will be the inverse ratio or reciprocal of each other. Thus the great lesson to be derived from the foregoing is, that 
the same amount of force is required in raising the body over the given space, whether the time be longer or shorter; or whether it be performed by the aid of the mechanical powers, properly so called, or not. Time, in waiting for the accomplishment of the object, necessarily waits until the amount of force or labor required shall have been expended in the accomplishment. Hence the whole amount of force expended in raising the body over the given space, will be the same, whether the time be longer or shorter, or whether the rate or intensity of the force be greater or less. If we attempt to investigate the law of falling bodies towards the earth, and to deduce therefrom the law by which the force of gravity varies in proportion to distance from the centre of gravity; or if we attempt to investigate the laws of force and motion as manifested in the revolutions of the heavenly bodies, and proportion the elements of force and motion to those of time and the space passed over, our labors will scarcely receive a liberal compensation unless we adopt unity as the standard of comparison. In an investigation of the law of falling bodies towards the earth, with a view to ascertain by what ratio the force of gravity varies with the varying distance from the centre of the earth or centre of gravity, some of the premises on which to proceed with the investigation, have been very properly assumed; as that of the comparison of two falling bodies towards the earth,- one commencing its fall from a state of rest, at or near the surface of the earth, and the other at some given number of semi-diameters of the earth from the centre of the earth; as that of four times the semi-diameter of the earth from the centre, or sixty times the semi-diameter of the earth from the centre, being the mean distance of the moon, \&c. And as the comparison of two bodies commencing their fall at different distances from the centre of gravity, is in most cases sufficient for all the essential developments required, they may be denoted by the letters $\mathrm{A}$ and $\mathrm{D} ; \mathrm{A}$ commencing its fall at or near the surface of the earth, which distance from the centre of the earth or centre of gravity, is called unity or $1,-$ while D shall commence its fall from several times the distance from the centre that A does, as at four times, eight times, \&c., or at sixty times, which is the distance of the moon, \&c., both commencing their fall at the same instant of time; and the operation to be performed and completed is for each to pass over an equal space, called the given space, whatever difference there may be in the time of the passage of the respective bodies over this given space.

The given space is some abstract numerical quantity, denoted by unity or 1, and may, if any prefer so to consider it, be denoted by some arbitrary measure, as one rod, that being very 
nearly the space over which a body will fall from a state of rest at or near the surface of the earth, in the first second of time after the commencement of its fall; or in which a body at the distance of the moon will fall in the first sixty seconds of time after the commencement of its fall. Such given space, however, should be some small quantity in which the intensity of the force applied to either $\mathrm{A}$ or $\mathrm{D}$, during the fall over the given space, shall not be increased; or so that there shall be no increase to affect our calculations and the results arrived at, or make them different from a perfect, uniform intensity, for so it will be considered.

It will be perceived that the object or final result of our researches is to ascertain the ratio between the intensity of the force of gravity applied to $\mathrm{A}$, and that which operates on D, during their fall over the given space. The given space to be passed over by each body, is an element in common between the bodies; and the operation or labor to be performed by the force of gravity, is to pass each body over the given space, or an equal space; and as the time of the passage will not be the same in respect to both bodies, we wish to ascertain what relation time has to the intensity of the force which urges the bodies over the given space; what relation the intensity of the force has to the acceleration of the bodies while passing over the given space, or to the instant velocity of the bodies at any given instant of the fall, or at any given point of the space; and hence, what is the ratio of the intensity of the force of gravity at the distance of $\mathrm{D}$ from the centre of gravity, to that at the distance $\mathrm{A}$; and hence, to ascertain the ratio or proportion which the whole amount of force expended upon $\mathrm{D}$ while passing over the given space, bears to the whole amount of force expended on A while passing over the given space. The premises upon which Sir Isaac Newton, Maclaurin, and other mathematicians, proceeded in their attempts to investigate the law of gravity, viz. to ascertain the intensity of the force of gravity as proportioned to the distance from the centre of gravity, - are based upon the very proposition that a body commencing its fall at or near the surface of the earth may be assumed at the distance of unity or 1 from the centre of gravity, viz. from the centre of the earth; and as the moon is sixty semi-diameters of the earth from the centre of the earth, hence a body commencing its fall at the distance of the moon, commences at the distance 60 from the centre of gravity; and a body at the surface of the earth will fall from a state of rest as far in the first second or moment of time, as the moon will fall from a tangent to its orbit in 60 seconds. These proportional distances of $\mathrm{A}$ and $\mathrm{D}$ are not objec- 
tionable otherwise than in the extension of a numerical formula adapted to so high a number as 60 , intended to aid the investigation by an exhibition of the numerical law of falling bodies in its most simple form, and in ringing those changes upon it best calculated to place the phenomena in the most perspicuous attitude.

I shall hence present the most simple numerical formula which I can conceive of, in which the time of the fall of $D$ over the given space will be eight fold that of $A$; or in which the distance from the centre of gravity at which $\mathrm{D}$ commences its fall, is eight times that at which A commences its fall. This will require that the given space over which $D$ is to fall shall be divider into 64 equal parts; and that the time of the fall of $D$ shall be divided into 8 equal parts, called moments, each moment being of an equal length to that employed by $\mathrm{A}$ in falling over the entire space. But it will be readily seen that the formula given may be extended so as to denote $\mathrm{D}$. at 60 times the distance from the centre of gravity that $\mathrm{A}$ is, viz. until the series denoted by the constant figure 1 shall amount to 60 places; thereby not only denoting the proportional distance of $D$ to that of A from the centre of gravity, but also the proportional time of the fall of $D$ over the given space to that of $A$, (for it will readily be seen that the proportional distance from the centre of gravity will always be as the proportional times of the fall over the given space.) And if the proportional distance or time of $\mathrm{D}$ to that of $\mathrm{A}$ be as 60 to 1 , the given space over which $\mathrm{D}$ shall pass will properly be divided into 3600 equal parts, (or into the square of the distance or of the time.) And hence in the last moment of the fall of $\mathrm{D}$, it will pass over 59 of the equal parts of the given space. The formula, then, as applied to $D$, may be as follows :

$$
\begin{array}{lllllllllllllll}
1 & & 1 & & 1 & & 1 & & 1 & & 1 & & 1 & & 1 \\
1 & 2 & 3 & 4 & 5 & 6 & 7 & 8 & 9 & 10 & 11 & 12 & 13 & 14 & 15
\end{array}
$$

Thus denoting the time or distance of $\mathrm{D}$ to be eight fold that of $\mathrm{A}$; and that the given space passed over by $\mathrm{D}$ is divided into 64 equal parts, the first part being passed over in the first moment, three equal parts in the second moment, five in the third moment, or in the time in which A would pass over the whole 64 equal parts. Now the number of equal spaces of the given space passed over by $\mathrm{D}$, is as well the square of the distance of $\mathrm{D}$ as of the time; for the distance is as the time, each being eight times that of $\mathrm{A}$.

The formula also presents this phenomenon: viz. at the end of the first moment figure 2 will denote the rate of velocity; 
figure 4 at the end of the second moment, figure 6 at the end of the third moment, and so on by the even numbers. Hence, at the end of the first moment the velocity would carry the body over two of the equal spaces in one moment, by a uniform motion, without the aid of further force applied; at the end of the second mornent the velocity alone, by a uniform motion, would carry the body over four equal spaces in one moment, without further force applied, and so on. If, then, the whole amount of force applied to $\mathrm{D}$, while passing over the given space, be divided into as many equal parts as the time is into equal moments, so that the several equal parts shall be denoted by the constant figure 1, then the velocity at the end of any given moment of the fall may be said to be twice the amount of the whole force expended from the commencement of the fall. If, then, the whole amount of force be thus divided into equal parts, we find that the whole number of those parts is the square root of the whole number of spaces into which the given space is divided; and hence that an equal amount of force is expended in such equal amount of time. Calling, then, the equal amounts of force in the equal moments of time, the forces expended during the fall over the given space, the sum of all the equal spaces into which the given space is divided is the square of the sum of the equal forces. Hence, the whole amount of space passed over at any given instant of time or at any given point of space during the fall, is the square of the whole amount of force that has then been expended during the fall; and hence the whole amount of the given space is the square of the whole amount of force expended while the body is passing over the given space; and this, too, whether the time be longer or shorter. Thus, passive time only waits for force to accomplish the labor, or object to be accomplished; and in such amount of time as shall be required according to the intensity of the force employed; and that 100 without the slightest interference on the part of time, the whole amount of the time of the operation only varying from unity in the reciprocal or inverse ratio to the intensity of the force, precisely in accordance with an operation by the mechanical powers (as of the wheel and axle) in raising a ponderous body.

Such, then, is the manifest operation of a falling body, which seems to be much more in accordance with all we know of the laws of force and motion, whether mathematically or experimentally obtained, than that hypothesis which has been so carelessly guessed off as the physical basis of astronomy, in which just fifty-nine sixtieths of the space passed over by a body commencing its fall at the distance of the moon, or distance 60 , is taken 
or withheld from the labor of active or effective force, and is credited to passive time, - thus making time far more active and effective than force, and far the greater operative. The given space, whether applied to A or D, is unity, and is the square of the whole amount of force expended during the fall either of A or D over the given space; and in unity, the roots and powers are equal; hence the amount of force applied to $D$ is equal to that applied to A, while passing over the given space, A being the standard, all its elements being assumed at unity; hence, the rate or intensity of the force applied to $A$ is as the whole time of the fall of A, (or figuratively; by the adoption of ratio in unity, the intensity of the force applied to A may be said to be in the inverse ratio or in the reciprocal ratio of the whole time of A's fall.) And so in respect to $D,-$ for as the intensity of the force varies from unity, it varies in the inverse ratio, or in the reciprocal ratio to that of the whole time; for such is the universal law or condition in respect to force and motion in time, or as applicable to time.

Here, then, is a principle which always holds good in regard to the effect of the force, (viz. the amount of labor performed,) whatever be the intensity of the force employed, - whether it be greater or less than unity, - for if the farther body be assumed at unity, the intensity of the force applied to the nearer will be more than unity; and the time of its fall, less than unity. The principle is this: in a falling body in which the velocity is constantly accelerated from the commencement of the fall, the amount of space passed over at any given instant of time, or at any given point of space, is the square of the whole amount of force which has been expended on the body from the commencement of the fall. And this principle holds good whether the body commence its fall at the distance 1 , or at the distance 60 , or at any other given numeral distance from the centre of gravity. Were it otherwise, the whole amount of force applied to a body while passing over a given space from the commencement of its fall, would always be in the inverse ratio to the distance from the centre of gravity. If the distance were 8 , the amount of force required to pass the body over a given space from a state of rest, would be but 1-8th of what would be required at the distance 1 ; so at the distance 60 , the amount of force required would be but $1-60$ th of what would be required at the distance 1; \&c. But such is the deduction of Sir Isaac Newton and his followers in respect to the law of falling bodies, and consequently in respect to the law of gravity, viz. the intensity of gravity as proportioned to the distance from the centre of gravity. But it is an operation, an effect produced by force, 
for which we are seeking, with a view to ascertain or deduce its proportional intensity at different distances from the centre of gravity; and although we may figuratively speak of the operation of time, of the effect of time, \&c. (not of the intensity,) nevertheless, such figures are not properly used or adopted in a mathematical investigation of those operations and effects which take place in time; and it would seem far more natural in an investigation of the intensity of gravity, that the space passed over at any given instant of time, or at any given point of the space from the commencement of the fall, should be referred to the active or operative element of force, rather than to the passive element of time; that the space passed over should be called the square of the whole force expended from the commencement of the fall, which is true in each individual case, (whatever the time may be,) rather than that the space passed over should be called the square of the time which has elapsed during the fall, - which can never be true, except the tirne be assumed at unity. For at the distance 1 , if the given space be the square of the whole time, at the distance 60 , the given space will be the square of only 1-60th of the whole time. Hence, if the time required at the distance 1 , for the body to fall over the given space, be increased sixty fold or to 60 times; in consequence of a body's commencing its fall at 60 times the distance, then to be sure, in order that the number of spaces shall be the square of the number of times, the given space at the distance 60 must be divided into 3600 equal spaces; nevertheless, a formula so constructed, in which the space to be passed over shall only be referred to the time of the passage, affords of itself alone no direct clue to the proportional intensity of the force of gravity at different distances from the centre of gravity.

Let us then always recollect, or keep in mind, the all-important principle, so full of meaning and so directly material to the issue, and which will disclose itself in an infinity of the most happy results and consequences, viz. that the space passed over at any given instant of time, or at any given point of space from the commencement of the fall, is the square of the whole amount of force which has then been expended during the fall; or in a falling body in which the velocity is continually accelerated by the force of gravity, the whole space passed over is the square of the whole amount of force that has been expended during the fall; nor should we forget the important principle that the rate or intensity of the force applied is in the inverse ratio, or in the reciprocal ratio to the whole amount of time in which the force is applied; nor the no:-less important principle, that the time is as the distance; all which principles 
are readily solved upon the simplest of the mechanical powers, as that of the lever, or wheel and axle; nor do we find that the immutable law based upon those simple principles, has in any case been abrogated or altered. And if these simple principles were once applied to the mechanism of the heavens, in lieu of that strange complication of human contrivance which now assumes the control, new beauties would be displayed, not only for the wonder and admiration of the learned, but for a vast multitude who have been wholly unable to discover much beauty in the works of creation through the medium presented.

Upon the foregoing simple principles we find this result; that if one body commence falling at the distance 1, at the same time that a body commences falling at the distance 60 , the velocity of the body at the distance 1 will be 3600 times as great as that at the distance 60 , at any given instant of time during their continued fall. For the force, viz. the rate or intensity of gravity, at the distance 60 , being only 1.60 th, or, .01666 , will be .0002777 , or the 1.3600 th part of the given space, which is the square of 1-60th part of the whole force applied to A while passing over the given space; the final result of which will be, that an equal amount of force will be expended on $\mathbf{D}$ while passing over the given space, that will be expended on $\mathbf{A}$ while passing over the given space. Nor can the operation be carried on otherwise, being established in the immutable fitness of things. Spite of all this, the Newtonian method of investigation makes the intensity of the force the inverse ratio of the square of the reciprocal of the time, as well as of the square of the reciprocal of the distance; all of which is equally absurd; and hence, he allows but 1-60th part of the force at the distance 60 , that is allowed at the distance 1 , to perform the same operation or same quantity of work. Thus, if one of the simplest and best established facts in creation is to be regarded, viz. that it is force which moves ponderous bodies, and that it requires a like amount of effective force to move a given body over a given space, whether it be in a longer or shorter time, if the time be only passive, the whole time standing as the reciprocal to the rate or intensity of the force applied, waiting its operation; then at the distance of the moon or distance 60 from the centre of gravity, the intensity of the force of gravity is 1.60 th as great as at the surface of the earth, or distance 1 from the centre of gravity. Thus the proportional velocities at any given instant of time of two bodies commencing their fall at the same time, but at different distances from the centre of gravity, are as the spaces generated, or rather, as the space generated by each respectively; and the whole space generated by either at any 
given instant of time, or point of space, is the square of the whole amount of force that shall have been expended in urging the fall thus far. If, then, the foregoing is a true exposition of the law of falling bodies, it is also at the same time a very easy and simple exposition of the law of gravity, determining that the rate or intensity of gravity varies inversely as the distance varies; and as it is demonstrable that such law of gravity would afford to the motion of the moon's apogee just four times the force afforded by the Newtonian law, (and consequently twice the motion,) the adaptation of such law would have saved Clairaut much perplexity in creating factitious force in accounting for the observed motion; and it would have deprived others of the privilege of creating factitious force whenever they found a revolving body not to quadrate with the Newtonian law. The Newtonian method, then, is erroneous. But many conspicuous names are engraved thereon, at an awful height; but those of Galileo and Kepler are not there; and whoever will search for those names must wander upon the rock far in the back-ground.

But the subject is vastly important; and it will be my endeavor to throw sufficient evidence before the public to induce a thorough investigation of the subject, even though I should be unjustly accused of resorting to the ingenious method of the painter for distinguishing the man from the bear, which he had painted on the same canvass. I would to Heaven that the world would investigate the Newtonian method of deducing the law of gravity; for if the world shall still prefer that the deduction shall be drawn from a consideration of the law of falling bodies towards the earth, in licu of being drawn from a far more natural and simple method, it is certainly proper that we should take heed to our steps, even"while travelling in the road which we may prefer.

I need not here refer to the well known laws of force and motion as applicable to the mechanical powers, as that of the wheel and axle, the windlass, \&c., in which the time is as the distance, or length of lever, where the force is applied, and in which the intensity of the force applied is inversely as the time; and consequently inversely as the distance. And it would seem, also, a mattegr of supererogation, to refer to the fact that the same law prevails in respect to bodies falling towards the earth; inasmuch as it is so directly manifest in the phenomena of falling bodies, as presented to view by the numerical formula by which the law is so admirably explained; and more especially, as it is fully assented to in that very process by which it has been supposed the 
fact has been deduced, that the force of gravity is inversely as the square of the distance; that is, that a body at the distance 60 , or at the distance of the moon, requires sixty fold the time in falling from a state of rest over a given space, that a body at the distance 1 or at the surface of the earth does. Hence the time is as the distance, by any and every consideration of the subject. Hence, if two bodies commence their fall at the same time at different distances from the centre of gravity, each to perform, describe, or pass over a given or like amount of space, each being acted upon by a uniform intensity of the force of gravity equal to the intensity by which it commenced its fall, the rate or intensity of gravity of each will be to the distance from the centre of gravity at which the fall commences, as it is to the time in which the body shall be falling over the given space; and this is universally assented to. Hence, it is as conclusively determined, in respect to falling bodies, that the time is as the distance, as it is in respect to the windlass or wheel and axle; and in respect to the windlass or wheel and axle, the whole time, as also the distance at which the force or power is applied, (or length of lever,) is inversely as the rate or intensity of the force applied at the given distance.

If, then, the rate or intensity of the force applied for accomplishing the object, namely, the urging of the body over the given space, be inversely, or in the reciprocal ratio of the time, it will also be in the reciprocal ratio of the distance. For as the distance is increased, the intensity of the force is decreased; (and for this reason alone is the intensity of the force decreased.) The intensity of the force is decreased in some given inverse ratio to that of time; or is decreased in some given ratio to that of the increase of time. This ratio is found to be, by every consideration which the subject has yet received or can receive, to be simply the reciprocal ratio of the time. And hence, to repeat, the time being as the distance, the intensity of the force of gravity decreases in the same ratio that the distance increases, and not in the same ratio as the square of the distance increases, as taught by the Newtonian hypothesis. Let $\mathrm{A}$ be conceived to commence its fall at the surface of the earth, or at the distance 1 , and we will suppose $A$ to fall over just 16 feet of space in one second of time (called one moment) from the commencement of its fall; and let D commence its fall at four semi-diameters of the earth from its centre, or at the distance 4 ; and the operation to be performed by $\mathrm{D}$ is to fall from its commencement, or from a state of rest, over 16 feet of space; and with a view to certain expositions, A and D shall commence their fall at the same in- 
stant of time; the 16 feet over which each body is to fall being called the given space, which given space is denoted by unity, or is that element in common between the two bodies which is denoted by unity. This is the task to be performed, and we wish to know the proportional amount of labor required in each case to perform the task. Now the 16 feet of space to be passed over by either $\mathrm{A}$ or $\mathrm{D}$, is unity or 1 ; the distance of $\mathrm{A}$ is 1 ; the time of $A$ is 1 ; the intensity or rate of force applied to $A$ is 1 ; the instant velocity of A, namely, the velocity of $A$ at any given instant of its fall while passing over the given space, is 1 ; and the whole amount of force applied to A while passing over the given space, namely, the whole amount of labor bestowed on A during its passage, is 1 . Hence each and every element taken. into consideration when applied to $A$, is assumed at unity. Hence, in respect to $A$, the given space, being unity, is the square of the distance, of the time, of the rate or intensity of the force, of the instant velocity, and of the whole amount of force expended on A during the fall over the given space.

The given space, to be passed over by $\mathrm{D}$, being also unity or 1 , is the square of 1-4th of the distance, or of the time; or is 1-16th of the square of the distance or of the time. If, then, the intensity of the force be inversely as the square of the distance or time, then the intensity of the force applied to $D$ will be 1-16th of the given space; and as the time of the application will be 4 , hence the whole amount of force applied to D during the fall over the given space will be 1-4th of that applied to $A$, and will be denoted by .25, or 1-4th of unity. But if the intensity of the force of gravity be imversely as the distance or time, then the intensity of the force applied to $D$ will be 1-4th as great as that applied to $A$, and as the time of the application of the force to $D$ is 4 , hence the whole amount of force applied to $\mathrm{D}$ during the fall over the given space will be unity or 1 , or just equal to that applied to A while passing over the given space. Let not, then, the middle of a formula, applied to D with a view to ascertain the proportion which its respective elements bear to unity or 1 , or to like elements when applied to A, perplex or thwart our calculations by considering the uniform series expressed by the constant figure 1, as only denoting time or distance, or rather as simply denoting time.

The uniform series then expressed by the constant figure 1 , as well denotes equal quantities of force expended in equal times, as it does the equal times into which the whole time is divided.

The great object of research, then, is directly this: to ascertain the ratio or proportion which the whole amount of force applied to $\mathrm{D}$ while passing over the given space, bears to the 
whole time of the fall over the given space; or what is the same thing, to ascertain the ratio of the amount of force expended in any given portion of the whole time, to that of the given portion of the time in which such given amount of force shall have been expended. But the given space is unity, as well when applied to $D$ as when applied to $A$, and it is an easy matter to divide the given space into equal parts, so that one of them shall be performed by $D$ in the first moment, (or 1-4th part of the whole time,) three spaces in the second moment, five in the third, and seven in the fourth. And such is the common presentation and extent of the explanation of a numerical formula designed to exhibit the law of a body falling from a state of rest towards the earth; and this is in fact all the exhibition required when but a single falling body is to be considered, or when there is no ratio to be determined between its elements and those of another falling body urged by a greater or less intensity of gravity; or rather, when the intensity of gravity is not brought into the calculation or investigation. But when the intensity or rate of gravity becomes a matter of consideration and inquiry; when the rate or intensity of gravity at different distances from the centre of gravity, is to be investigated by means of a consideration of the law of falling bodies, and in which the time (being as the distance) must be proportioned to the force, and which, consequently, requires a comparison of two bodies, commencing their fall at different distances from the centre of gravity; - a consideration of the formula of $\mathrm{D}$, which only adapts the equal parts of unity, of which the given space is compared, to the number of equal moments in which $\mathrm{D}$ shall be falling over the given space, will not be likely to lead to any satisfactory result in respect to the ratio which the intensity of gravity bears to the time of the fall over the given space, and consequently, the ratio of the intensity of gravity, as proportioned to the distance.

The uniform series of the formula for $\mathrm{D}$, expressed by the constant figure 1 , always denotes the distance at which $\mathrm{D}$ commences its fall, (that of A being unity;) it also denotes the units of time (called the times) in which $D$ is passing over the given space, which is unity; the time in which $\mathrm{A}$ is falling over the given space, being unity or 1 . So the uniform series expressed by the constant figure 1, most emphatically denotes the number of equal quantities into which the whole amount of force expended on D during its fall over the given space, is divided; in which case, one of the equal quantities of the whole force expended during the fall, will be expended in one of the equal times ; for the intensity of the force is properly considered as uniform as the time. Hence, what may properly be termed the accelerating series of 
the formula, namely, the natural numbers in their order, or the odd numbers in their order, applies equally well to the uniform series when it denotes force as when it denotes time; and the sum of the odd numbers of the accelerating series will be as well the square of the forces into which the whole force is divided, as of the times into which the whole time is divided, - as also of the distances into which the whole distance is divided, (the tim's and distances being but so many equal units of the time and distance of A.) Thus each of the times or of the distances denoted by the uniform series, is unity, or is numerically equal to the entire given space to be passed over. Hence, in respect to time and distance only, an equal division of the given space into the sum of the odd numbers in the accelerating series, would seem to be of but little consequence, so far as the main question is concerned; such being the simple consequence of ratio and proportion, (which always accompany unity of purpose, ) the beneficial results of which are manifested by an application of such equal division of the given space, to an equal division of the whole amount of force expended on the body while passing over the given space; in which case the amount of force denoted by the first figure 1 , in the uniform series, is expended on the first space in the accelerating series, the next amount of force upon the next three spaces, and so on; in which case the whole amount of force, whatever be its ratio to that of the time or distance, or to unity, is expended in the given space. In such case the amount of space at any given instant of time, or at any given point of the space, generated by the force, (not by the time or the distance,) is the square of the whole amount of force which has been expended from the commencement of the fall; and consequently it is so at the end of the given space, or when the amount of space generated has become unity. Let $\mathrm{D}$, then, commence its fall at the distance 4 , and let the given space for $\mathrm{A}$ and for $\mathrm{D}$ be 16 feet, and let them commence their fall at the same instant of time; then, when A has fallen over 16 feet, $\mathrm{D}$ will have fallen one foot. When $\mathrm{D}$ has fallen over 16 feet, its velocity will be equal to that of $A$ when $A$ has fallen over 1 foot; and when D has fallen over 16 feet, its velocity will be one-fourth as great as that of $A$ when $A$ has fallen over 16 feet. At any given instant of time while $A$ is falling over the given space, its velocity will be sixteen-fold that of $D$ at the same instant. At any given point of the given space, as at the end of the first or second foot, \&c., the velocity of A will be four-fold that of $D$, at a like point of the given space; hence $D$ must fall over 16 times a given space, in order to acquire the velocity of $A$ at the end of such given space. As the time of $A$ 
is 1 , and the time of $\mathrm{D}$ is 4 , and as the whole time only stands as the reciprocal of the intensity of the force, waiting its operation, the same amount of force will be expended on $D$ as upon A while passing over the given space.

It is easy to conceive upon what ground an error might occur, in an attempt to deduce the law of gravity from a consideration of the law of falling bodies, namely, by not properly considering or regarding the simple principle or axiom, that in a falling body there is no motion, not even the least, without acceleration; namely, that the whole motion is an accelerated motion. Consequently in the first moment of the fall, however short we may conceive such moment to be, if it be but time, the motion is accelerated therein. If, however, in the consideration of a formula, we conceive the first moment (and consequently the first space, however short or small) to be performed without acceleration of motion; and of course upon a totally different principle from that which occurs in the remainder of the time and space, - in such case it would at least become problematical whether the intensity of the force of gravity should be assumed as being inversely as the square of the distance, or inversely as the distance. For nothing would occur in the first moment or first space, to make the amount of space then passed over, the square of the amount of force then expended; for this principle arises wholly from the acceleration of the motion. But if there were no acceleration of the motion in the first moment and space; if in the first space the motion is to be uniform, and only to be accelerated in future ; then it might well be conceived that the force is as the motion in the first space or first moment; and consequently that in the first space and moment, the intensity of the force of gravity is inversely as the square of the distance, or of the whole time; for surely, in every formula, the first space passed over is but one of the whole number of equal spaces into which the given space is divided, which makes the square of the distance from the centre of gravity. But if such error be once adopted, (and a more manifest error can scarcely be conceived, the consequences and results must necessarily partake of the same error. And as the force is to be uniform throughout the fall over the given space, a like amount of force as that expended in the first moment will be expended in the next moment of equal length, and while the body is passing over three times the space, in consequence of an accelerated motion acquired in the first moment, and while by supposition the motion was uniform. The fact, however, is too manifest for comment, that if we assign some given moment of time, as one second for instance, to the first space in any given formula, such moment being assumed at unity, the first space, how- 
ever small, may also be assumed at unity ; in which case the aistance, the rate of force, the rate of instant velocity, and the whole amount of force expended while passing over such space, will necessarily be assumed at unity. Hence the first term in what is called the uniform series, and the first term in what is called the accelerated series, always form a perfect formula for the standard body, or the one commencing its fall at the distance 1, by which to compare the elements of another; they being but abstract numeral quantities assumed at unity.

Thus the distance of the moon may as well be assumed at unity as the surface of the earth, and become the standard by which to compare another body commencing its fall at sixty times the distance of the moon from the centre of the earth. Nevertheless, the motion of a body commencing its fall at the distance of the moon, will be accelerated in its fall in the first second of time, or in any shorter time; and so will that of the body commencing its fall at sixty times the distance of the moon, however small the space may be which is passed over in the first second of time.

If then the motion of the body be accelerated while passing over the first space, (and no one will assume that it is not,) then the force of gravity is inversely as the distance from the centre of gravity.

Perhaps a still more simple exposition than has been given of the formulas denoting the law of falling bodies towards the earth, may more readily suggest that comparison of the elements to be considered, from which to deduce the force of gravity as proportioned to distance from the centre of gravity, than any which I have heretofore presented.

When two bodies are conceived to commence their fall at different distances from the centre of the earth, or centre of graviity, with a view of comparing the various elements of the two bodies, they are conceived to commence their fall at the same time, and one of them (called the standard) is conceived to commence its fall at the distance of unity, or 1, from the centre of gravity, whether it commence its fall at the surface of the earth or elsewhere, and the other to commence its fall at some given number of units from the centre of gravity, as at the distance 4, 8,60 , or any other given distance ; and the space to be performed or passed over by each, as the entire operation of each, is to be equal one with the other, and is assumed at unity, or 1, in respect to either body. Such space is called the given space; and may, if any prefer to consider it in our arbitrary measures in preference to abstract numeral quantities, be assumed at 16 feet; and that the body at the distance 1 , will pass from a state of rest 
over the given space, in consequence of the force of gravity, in one second of time, permitting a second of time to be called a moment, when used in the investigation, which moment is to be understood as being one second of time.

As each and every element of the body which commences falling from the distance 1, is properly or necessarily assumed at unity, hence the formula for such body can only consist of two figures, each denoting unity, thus:

1

the lower figure denoting the given space to be passed over, and the upper figure denoting the second, or moment of time, in which the space is performed; also the rate or intensity of the uniform force with which the body is urged, as also the whole amount of force applied to the body while passing over the given space, or the given moment of time. If a body commence its fall at the distance of 4 from the centre of gravity, its formula will necessarily be thus :

\section{$\begin{array}{llll}1 & 1 & 1 & 1\end{array}$ \\ 1234567}

In this formula the given space is divided into sixteen equal parts, denoted by the sum of the odd numbers contained in the lower or accelerated series; while the time of the passage of the body over the given space is increased to four units, denoted by the four figures in the upper series; that is, the time being as the distance, is in this formula increased to four seconds, or moments. So also the upper series may denote an equal division of the whole amount of force expended during the fall over "the given space, into four equal parts ; the first part to be expended in the first moment; the second part in the second moment, and so on. Hence one fourth of the whole amount of force will be expended in one fourth of the whole time; and one foot of the given space, or one sixteenth of the given space, will be passed over in the first moment, or in one fourth of the whole time; three feet in the second moment, five feet in the third, and seven in the fourth, in which case the number of equal spaces into which the given space is thus divided, is the square of the number of seconds or moments, or units of time in which the body is falling over the given space, or over unity, as also the square of the number of equal parts into which the whole amount of force expended during the fall is divided. It is then the whole amount of the force expended while the body is falling over the given space, denoted by unity, that we are seeking, and consequently we wish to obtain its rate or intensity at the distance 4, compared with that at the distance 1 , or compared with unity. 
Thus if at the distance 4 the rate be but 1-16th of unity, then it is inversely as the square of the distance. If at the distance 4, its rate be $1-4$ th of unity, then it is inversely as the distance. Thus at the distance 4 , the rate of force is in some given inverse ratio to the whole distance, or whole time; and whether it be in the inverse ratio to the first or second power of the whole time, or whole distance, (for the time is as the distance,) is the question.

It will be readily seen that the formula for a body that commences its fall at the distance 4 , is a perfect epitome of a formula for a body commencing its fall at a greater distance than 1 . Nevertheless if the formula be ever so far extended, as for instance, if the body be conceived to commence its fall at the distance 60 , in which case the uniform series expressed by the constant figure 1, will be extended to sixty places, (denoting the number of unit distances, and also the number of seconds or unit times,) still the space to be passed over is unity, notwithstanding it is divided into as many equal parts as unity is contained in the sum of the odd numbers found in the second or accelerating series, namely, 3600 , if the distance or number of seconds or moments be 60 . The accelerating series then divides unity into equal parts, but does not increase or decrease it, while the uniform series denotes the increase of the units of time from that of one only, which is required when the distance is 1 , to that of the number of figures in the uniform series. Each figure, then, in the uniform series, so far as it refers to time or distance, is unity.

'The left hand figure, or figure 1 , in the accelerating series, always denotes an amount of space equal to the reciprocal of the square of the distance, or of the time in which the body is passing over the given space or the whole space to be passed over, namely, unity; nevertheless, the time of passing over the amount of space denoted by figure 1 in the accelerating series, is unity; however small such space may be; and it is manifest that by extending the distance such space may become exceedingly small. Nevertheless, if in the first moment of the fall there be motion, (and the fall implies motion,) then space is passed over in the first moment of time, or in one second of time, however small such space may be.

Now it may be set down as a lemma, or even an axiom, that in a falling body there is no motion without a corresponding acceleration of the motion; and that the same force which causes the motion, at the same time causes the corresponding acceleration of the motion. Hence if a body commence its fall from ever so many units of distance, and consequently the unit of 
space to be passed over be divided into ever so many equal parts, (the number of which is always the square of the number of units of distance or of time,) the motion of the body while passing over the first equal part of the given or unit space, namely, that denoted by the left hand figure in the accelerating series, will be constantly accelerated.

Hence the uniform series, expressed by the constant figure 1, may be used to denote the rate or intensity of the force, not as units of force, as it does units of time or of distance, but in some given reciprocal ratio to that of the whole time or the whole distance, as that of the simple reciprocal ratio, or as the square of the reciprocal ratio of the whole time or whole distance.

If the rate of force be in the simple reciprocal ratio of the whole time or whole distance, namely, inversely as the whole time or whole distanee, then the whole amount of force expended while the body is falling over the given space, namely, unity of space, will be unity of force; that is, the whole amount of force expended will be unity, and the amount of space passed over will be the square of the whole amount of the force then expended. In such case the motion of the body will have been accelerated, throughout the whole given space, as well in that part denoted by the first figure in the accelerating series, (however sinall or great, whether it be the $1-16$ th part or the 1.3600 th part,) as in any other part of the given space. That is, at any point of the given space, or at any instant of the fall, the amount of space then passed over, however small a portion of unity it may be, will be the square of the amount of force which has been expended. But if the rate or intensity of the force be the reciprocal ratio of the square of the whole time or of the whole distance, namely, inversely as the square of the whole time or of the whole distance, then the whole amount of force expended on the body while passing over the given space, namely, over unity of space, will be the simple reciprocal ratio of the whole time, or whole distance, (thus giving to the whole amount of force expended during the fall, the same numerical quantity, or the same ratio compared with unity, that should be given to the intensity,) in which case, at the distance 60 , the amount of force required to urge the body over the given space, would be but $1-60$ th of what would be required at the distance 1 .

Now, a more manifest proposition can scarcely be stated or considered, than that such a principle wholly prohibits acceleration to the motion of the body while passing over the first space, or that denoted by the first figure in the accelerating series, nowever great or small such space may be, and only permits acceleration to take place in future; and hence the first space passed 
over is, by such a course of reasoning, treated in the same manner as the given space passed over by the body falling from the distance 1 , and in the time 1 , and in which the intensity of the force, as also the whole amount of force, are respectively assumed at unity, and in which the element of acceleration is not necessarily an element to be considered. If, then, the motion over the first space be not accelerated, but uniform, then the intensity of the force while the body is passing over the first space, (however great or small,) is as the motion of the body moving; but if the motion be accelerated, then the intensity of the force applied is not as the motion of the moving body. The consequence then is most obvious; for if we assume the motion to be uniform in the first space, however small, we necessarily make the intensity of the force inversely as the square of the time, or of the distance; and consequently the whole amount of force expended while the body is passing over unity, or the given space, will be the inverse of the distance, or of the time.

For the amount of force expended in the first space, (however small such space may be,) is the standard or criterion by which to compare the future acceleration; for the intensity of the force being uniform, a like amount of force to that expended in the first space will be expended in the three succeeding spaces, or in the next five, and so on.

Hence the advocates of such hypothesis must say that the same amount of force which produces a uniform motion over the first space; (however great or small such space may be, will produce an accelerated motion over the three succeeding spaces, - and so on. The simple consequence then is, if the motion in the first space be uniform, the rate or intensity of the force will be inversely as the square of the distance. But if there be no part of the given space in which the motion is uniform, or not accelerated, or in which the rate or intensity of the force applied is as the motion of the falling body, then the ratio or intensity of the force applied is inversely as the whole distance, or the whole time of the fall over the given space.

Mr. Vince says that "Sir Isaac Newton found that if the force with which bodies fall upon the earth's surface were extended to the moon, and to vary inversely as the square of the distance from the centre of the earth, it would in one minute draw the moon through a space which is equal to the versed sine of the arc which the moon describes in one minute. He concluded, therefore, that the moon was retained in its orbit by the same force as that by which bodies are attracted upon the earth." That the power of attraction, which causes bodies to fall to the earth, extends to the moon, and retains it in its orbit, 
is perhaps too manifest for any one to doubt; and this was the conclusion of Kepler long before the time of Newton. But how Sir Isaac Newton should have come to such a conclusion from his observations and calculations may appear somewhat mysterious.

Now that the versed sine of the are which the moon would d scribe in its orbit in 60 seconds of time, is equal to the amount of deflection from a tangent in the same time, all will agree. This part of the subject I have before partially examined; and the reason for preferring the method of considering the amount of deflection from the tangent, in preference to the versed sine of the arc, is because it gives a much more comprehensive range for investigation.

Now I will not undertake to say but that the versed sine of the arc passed over by the moon in 60) seconds, is equal to the fall of a body from a state of rest near the earth in one second; to be sure the space passed over in 60 seconds is, in respect to the circumference of the moon's orbit, a very litlle distance; and so far as could be well ascertained from observation alone, would not present an error that could readily be detected by observation. Nevertheless, such little distance would be sufficient to contain a mathematical error, vast in its consequences, if it existed. But it must be presumed that Sir Isaac Newton drew his conclusion, that the attraction of the earth extended to the moon, from some calculation made upon said observations; for otherwise, he might as well have supposed that Jupiter or Saturn was retained in its orbit by the attraction of the earth. But Mr. Vince says that Sir Isaac Newton found, \&c., but the elements upon which he found are not given by Mr. Vince, and bence it is fair to conclude that they are the same that are given in other places, by which it is said the law of gravity was discovered to be inversely as the square of the distance, viz: that the moon being 60 times as far from the centre of the earth as the surface is, would be deflected as far from a tangent to its orbit, in 60 seconds of time, as a body near the surface of the earth would fall from a state of rest in one second of time; and that this amount of deflection from a tangent would be equal to the versed sine of the arc of the moon's orbit which it would pass over in 60 seconds, and which induced the conclusion, not only that the attraction of the earth extends to the moon, but that it varies inversely as the square of the distance. Hence, the statement of Mr. Vince may be resolved into this : that Sir Isaac Newton, according to his calculation, found that if gravity decreases inversely as the square of the distance, that a body falling from a state of rest directly towards the earth, at the dis- 
tance of the moon, would fall as far in 60 seconds, as a body at the surface of the earth would in one second, and that the amount of such fall in 60 seconds would be equal to the versed sine of the are which the moon would pass over or describe in the same time; or equal to the deflection of the moon from a tangent in the same time; and we must add also, that the moon is 60 times as far from the centre of the earth as the surface of the earth is.

Now that there is error in all these positions except that of the distance of the moon from the centre of the earth, may perhaps be safely alleged upon the truth of mathematics, geometry and the law of falling bodies.

I am aware that Mr. Vince's statement of what he and others suppose Sir Isaac Newton to have found, is rather vague and intangible; and that if a proposition be drawn from it for the purpose of demonstration, either by way of reductio ad absur. dum, or otherwise, certain things not contained in the statement of Mr. Vince, must be contained in the proposition; as that the moon is sixty times as far from the centre of the earth as the surface of the earth is. Then the proposition may be put thus, viz: If gravity decreases from the centre of the earth to the distance of the moon, inversely as the square of the distance, and a body at the surface of the earth will fall from a state of rest 16 feet in one second of time, how far will a body, at the distance of the moon, fall from a state of rest, in 60 seconds; or how far will the moon be deflected from a tangent to its orbit in 60 seconds? And it appears to me that this proposition is so far based upon the statement of Mr. Vince, as, when solved, to determine what Sir Isaac Newton found, unless he found something that does not exist. Inasmuch as the dimensions of what he found are not given by Mr. Vince, either in length or breadth, we must endeavor to deduce them as well as we can from the premises; and first, as to the distance a body would fall in 60 seconds, from a state of rest, at the distance of the moon, or at distance 60, upon the assumption that gravity varies inversely as the square of the distance from the centre of the earth to the distance of the moon; and that a body falling from a state of rest at the surface of the earth will fall 16 feet in one second of time; and also that the amount of fall at the distance of the moon is to be determined by the law of falling bodies. Upon the Newtonian law of gravity; then, a body falling from a state of rest, at the distance of the moon, would fall in 60 seconds of time the 1.3600 th nart of 16 feet, or a little more than $1-20 \mathrm{l}$ h of an inch; in which case it would fall in the first second of time the $1-12,600,000$ th part of 16 feet. It is a very little distance, to be 
sure, compared with the time, but it is all that the law of falling bodies will allow upon the Newtonian hypothesis of the law of gravity. This quantity then, viz: the $1.3660 . h$ part of 16 feet, according to said proposition, would be Sir Isaac Newton's versed sine of the are which the moon would describe in 60 seconds; and consequently, according to his calculation, would be the amount of deflection of the moon from a tangent in the same time. Nevertheless, a body at the distance 60 will fall the 1-3600th part of 16 feet in the first second. Let us now suppose that a body at the surface of the earth, viz: at the distance 1 , will fall 16 feet from a state of rest in one second of time; and that a body at the distance of the moon, viz: at the distance 60 , will fall 16 feet from a state of rest in 60 seconds of time, which are the true proportions. This proposition will at once shift the supposed law of gravity from the inverse of the square of the distance, to the inverse of the distance, or to the law as it is, if it be in anywise corroborated by the law of falling bodies: In such case, the amount of fall at the distance 60 in the first second of time, will be the 1-3600th part of 16 feet; in the next second, the 1-1200th part; in the third second of time, the 1.720th part of 16 feet; and so on; thus increasing in each successive second of time in the ratio of the next succeeding odd number; and in which case, the whole 16 feet would be accomplished in the 60 seconds, in accordance with the law of falling bodies. But no one can rationally suppose that gravity (which is as uniform at any given distance as time is) will, at the distance 60 , exert three times the force in the second second of time that it does in the first; five times as much in the third, and so on, any more than it will at the distance 1 , or at the surface of the earth; or than time will; which it must do, if gravity be inversely as the square of the distance, in order that a body at the distance 60 may fall as far in time 60 , as a body at the distance 1 , will fall in time 1 . But this is palpably plain, and it being within the reach of every person to examine and ascertain the truth for himself, mankind should not have been left in an error in respect to it for a moment.

I will acknowledge that in respect to the extent of what it is supposed Sir Isaac Newton found, it is left somewhat vague and intangible by Mr. Vince. Nevertheless, mankind have got the idea, or notion, that he found that the moon will be deflected as far from a tangent in fi0 seconds of time, as a body would fall from a state of rest in the same time, at the distance of the moon. For it is said that the moon would be drawn towards the earth by gravity, in 60 seconds, through the versed sine of the arc that the moon would describe in that time in her orbit. 
This is a serious allegation; and when taken implicitly, and without examination to see if these things are so, or can be so, is well calculated to lead into a wilderness of error, in lieu of leading to the truth in matters depending upon such premises. And $I$ am confident the error contained in the above statement of what Sir Isaac Newton found, has been 100 long continued for the benefit of philosophy.

The rate of convergency of a planet is inversely as the period. What may be called deflection in time, (so far as rate may be said to apply to deflection.) is inversely as the square of the distance, or equal to Sir Isaac Newton's rate of force. And the rate of deflection in space is inversely as the distance, or the square root of the rate of deflection in time. Hence the rate of convergency is a mean proportional between the rate of deflection in space and the rate of deflection in time. But as the factitious element of deflection in time and space requires a more labored explanation, we will not dwell upon it here. But I will here make the allegation that no one will deny, namely, that the greater the mean distance of a planet of the same system is, the greater will be the motion in proportion to the rate of gravity or force; and the less will be the rate of convergency in proportion to the rate of gravity. For it is only upon this principle that the squares of the periods of a system of planets can be as the cubes of their mean distances from the sun. Thus, upon the hypothesis that gravity is inversely as the distance, the mean rate of gravity will always be a mean proportional between the mean rate of motion, and the mean rate of convergency ; and in the same ratio that motion increases in proportion to the rate of force as the distance increases, so the rate of con. vergency decreases in proportion to the rate of gravity. Thus at the distance 4 the rate of motion is twice as great in proportion to the rate of gravity as at the distance 1 ; and the rate of convergency is half as great in proportion to the rate of gravity as at the distance 1. That is, by the hypothesis that gravity is inversely as the distance, the rate of motion increases upon the rate of gravity as the square root of the distance increases from unity; and the rate of convergency decreases upon gravity as $t$ e rate of motion decreases from unity.

But upon the hypothesis that gravity is inversely as the square of the distance, the rate of motion will increase upun the rate of gravity as the period increases from unity. Thus if the distance be 4 , the rate of motion will be eight times the rate of gravity. And upon such hypothesis of gravity, the rate of convergency will increase upon the rate of gravity as the distance increases, in the same ratio that the square root of the distance in- 
creases from unity; so that when the rate of motion is eight times the rate of gravity, the rate of convergency will be twice the rate of gravity. Hence the mean rate of motion is to the mean rate of convergency as the mean distance is to unity, by any law of gravity; nor do the mean rates of motion and convergency, as proportioned to each other and to the distance and period, depend upon our knowledge of their proportions, or upon our knowing how gravity is proportioned to distance. But the reason for introducing the foregoing principles in respect to the proportion between motion and convergency, is for the purpose of showing that the motion of a planet in its orbit counteracts convergency; that is, if the mution be increased in proportion to the force or gravity, the rate of convergency will fall short of the rate of motion in the ratio of the distance to unity by either hypothesis of force. And hence, as the distance is increased, the motion becomes greater, in proportion to the force, and also greater in proportion to the convergency by either hypothesis of gravity. So then at the distance 4 the convergency of a body will not be as great in proportion to the motion as when the distance is 1 . The consequence then is apparent, that as the distance is increased, not only the convergency but the deflection is less in proportion to the motion. This becomes apparent if we take a planet "hose distance is 1 , and another whose distance is 4 , and conceive the planet whose distance is 1 , to have passed over $1-4$ th of its orbit, and the planet whose distance is 4 , to have passed over $1-8$ th of its orbit, or over twice the space of the other, and in quadruple the time; in which case, the deflection of the planet whose distance is 1 , is unity, while the deflection of that whose distance is 4 , is 1.171576 , in which case, if deflection were to proceed upon the principle of falling bodies, when the time becarne doubled, - namely, when the planet whose distance is 4 had passed over 1-4th of its orbit, - the amount of deflection would be 4.686304 , when in fact it is but 4 . Hence the deflection was not three times as great in the last half of the time as it was in the first; and hence deflection does not correspond with the law of falling bodies.

I have made the example broad for the purpose of showing the error in the expanse. Nor is the error mathematically avoided, by supposing a very little time to have been passed over; as that passed over in 60 seconds of time by the moon. And hence, Sir Isaac Newton did not find, by any law of gravity whatever, that the versed sine of any small are of the moon's orbit would be the distance over which a body would fall from a state of rest at the distance of the moon, while the moon was describing or passing over such arc. We hence find that the deflection of a planet, or fall from a tangent, is not likened in its 
progression to that of falling bodies; and hence, as an element for deducing the law of gravity, it is illy qualified, being too foreign and factitious. It may, however, be proper, inasmuch as so much reliance has heretofore been placed upon it, to give it still further consideration, in which not only its futility as such element may be shown, but many exarnples given by which it will be shown that most of the deductions which are apparently drawn from its consideration are mere fictions, and not realities. This is the case in a previous section, notwithstanding it presents much important truth; and such will consequently be the case in the remainder of the present section so far as deflection is treated of upon the principle that it is one of the legitimate elements of the planets and their orbits; and hence the reader must not consider it as an element proper, but that most of the apparent results drawn from its consideration are fictions; however much it may serve as a discipline designed to exhibit specious errors, for the purpose of avoiding thern.

I have said that deflection may be properly divided into deflection in time and deflection in space; and that the rate of deflection in time, is the square of the rate of deflection in space. For example: Take the planets $\mathrm{A}$ and $\mathrm{C},-\mathrm{A}$ at the distance 1 , and $C$ at the distance 4 , and let them pass from their tangents some little, but equal time; in which case $C$, will have passed over one half the space that $A$ has; and according to the commonly received notions of deflections, (which, however, we have shown to be erroneous,) A will have fallen sixteen times as far from its tangent as $\mathrm{C}$ has. And such is what I have thought proper to call deflection in time.

Suppose A should stop, and C pass on over equal space in its orbit that $\mathrm{A}$ has, or twice the time that $\mathrm{A}$ was passing over equal space, (which I have thought proper to call the deflection in space, or the deflection of two planets while passing over equal space.) Thus, if it were the case, that in equal time, the deflection of C was 1-16th that of $\mathrm{A}$, and while passing over equal space was 1-4th that of $\mathrm{A}$, the deflection of $\mathrm{C}$ in time, would be the square of its deflection in space. Hence, I have made the allegation upon such hypothesis; and in such case, the deflection in time would be the inverse of the square of the distance, while the deflection in space would be the inverse of the distance.

If the deflection of $\mathrm{C}$ at the end of equal time, were 1-16th of that of $\mathrm{A}$, and if its fall from a tangent were governed by the law of falling bodies, then, at the end of equal space, its deflection must necessarily be 1-4th that of $\mathrm{A}$. But we have shown that such are not the facts. It is then but simple hypothesis, based upon the erroneous supposition that deflection is identical in its construction with that of falling bodies. 
We thus perceive that we are dealing in hypothesis, the results of which are fictions, and not those realities which are material to the issue; or at least those fictions are but collateral to the issue. But let us pursue a little the consequences arising from the foregoing hypothesis; and to this end I will take A, and another planet, at distance 60 , denoted by $\mathrm{D},-$ or a body revolving at the surface of the earth, and another at the distance of the moon.

Now their relative motions will be as 1 to .12909 , or inversely as the square roots of their respective distances. And when the spaces passed over by the two bodies in their orbits are as the square roots of their respective distances; or when the times passed over are as their respective distances; then, according to the notions which the world have entertained in respect to the correspondence between the deflection of a planet and the law of falling bodies, the deflection or fall from a tangent will be equal in the two bodies; and in such case when the spaces passed over in their respective orbits are as 1 to 7.74595 , the deflection of one will be equal to that of the other.

But however plausible such hypothesis may appear, it is nevertheless untrue in lact, and should not be permitted to lead into unbounded errors.

Recur again for explanation to the planets $\mathrm{A}$ and $\mathrm{C}$; and upon the hypothesis aforesaid, if $\mathrm{C}$ had passed over equal space with $A$, (and in twice the time,) its deflecion would be 1-4th that of $A$; the consequence of which would be, when $C$ had passed over twice the space of $A$, its deflection would be equal to that of $A$, viz., when the spaces passed over were as the square roots of their distances, or the times passed over were as their distances. For if deflection be like the law of falling bodies, then, if the deflection of $\mathrm{C}$, in equal time, be 1-16 th that of $\mathrm{A}$, it will be $1-4$ th in equal space, and in quadruple the time and double the space, it would be equal to that of $A$. In which case when $\mathrm{C}$ has passed over $1-8$ th of its orbit, its deflection should be just equal to that of $A$ when it has passed over 1-4th of its orbit, which is not the case; and hence those proportions do not hold in the deflection of two planets, neither in the expanse, nor in any little distance.

Upon the foregoing erroneous hypothesis, the same amount of gravity expended upon each of two planets of a system (if gravity be inversely as the square of the distance) would give the same amount of deflection in each; and sixteen times the amount of gravity or rate of gravity, would give twicc the motion. But if gravity be inversely as the distance, then the same amount expended on two planets of a system will give the same 
amount of deflection, if the times in which it is expended are as the distances; and four times the amount of gravity will give double the motion. But the results are neither of them correct as to deflection.

Thus in respect to the factitious and troublesome element of deflection, whether considered in reference to time or space, it furnishes but little useful information in respect to the laws of force and motion; as it does not correspond with the law of falling bodies, as has been too implicilly believed. To be sure, from: an attempt to get the rate of deflection in time, we obtain an expression, denoting the inverse of the square of the distance; and by attempting to consider, or obtain, the rate of deflection in space, we obtain an expression denoting the inverse of the distance; both those expressions, we perceive, however, are wholly founded upon assumption. The fact is, there has been a mistake in supposing that the progress of the deflection of a planet from a tangent, caused by the progress of a planet revolving in its orbit, was identical with, or corresponded with the fall of a body from a state of rest. And, but for this mistake, the world might have been saved much trouble. For it has introduced into the science of astronomy that kind of scribe rule, by which things must be cut and tried until they fit; so that already have they been compelled to equate some thirty supposed irregularities of the moon, said to be the legitimate offspring of physical astronomy, resulting from Clairaul's reconciliation with the Newtonian law of gravity; which reconciliation was brought about by more complicated ingenuity than most astronomers and mathematicians are willing to reinvestigate. For having adopted an hypothesis in respect to the law of gravity, which would give but just half the motion to the apogee of the moon which it actually has; and being compelled to supply the deficiency of gravity from extraneous materials; the moon has in consequence been thrown into more perturbations than ever was a hunted hare; while those who have essayed to pursue, have been more sagacious than a pack, in tracing out and exploring her devious paths caused by her perturbations.

I must confess, that I never have been reconciled to the idea that the universe was all an error; and that truth was only to be found at its furthest extremity, after groping through eternal darkness; nor am I well satisfied with that class of truths which are supposed only to be found in those ultimate ratios which are supposed to end in nothing; but I prefer that ratio should have, at least, a numerical point to stand on. But I know, not of a more notorious instance of the adoption of the principle, 
that the path of eternal error is the road to truth, than that evinced in the modern researches for the quadrature of the circle; in which not a single step that is taken in the progress (at least, after taking up the line of march) develops or discloses a single fact material to the issue. Neither the area, nor any linear measure of a single polygon is measured, determined, or known.

The summing up of the process at any stage of the proceeding comes out error. Hence, by such method we have no criterion by which to determine whether we are or are not in the right path. There is no progression or succession of results, which serves constantly to confirm us in the correctness of our pursuit, no landmarks by the way to cheer us onward, and denote the progress we have made, no pleasant reminiscences, like those of Homer's

"Wayfaring man; who wanders o'er

In thought, the length of road he trod before."

There is no series of results arising from the application of the powers and roots of numbers, denoting demonstration, which should as fully and conclusively determine the final result as any other in the process.

Our whole hope and consolation in the process seems to arise from the circumstance, that the farther we carry it, if we sum up our accounts, so much nearer than at any previous step we find we are to what we conceive to be the neighborhood of truth, and we hence conclude that the path we are pursuing leads directly there; and that truth will be found at the terminus of our route. But this is supposition; it is not conscious truth; and how near the terminus of the process the truth lies, we know not. And should it eventually be found that such process is wholly erroneous, it may serve to weaken the faith of mankind in the belief that truth is only to be sought and obtained through an infinite series of errors.

I will now conclude this section by a few desultory dictums and remarks.

The mean distance of a planet divided by the period, gives the rate of mean motion. The distance divided by the rate of motion gives the period. Hence, when the distance is 1 , the rate of motion is equal to the period. When the distance is 2 , the rate of motion is 1-4th of the period. When the distance is 3, the rate of motion is 1-9th of the period, and so on.

If gravity be inversely as the distance, the period divided by the distance will give the amount of gravity expended during the period. If gravity be inversely as the square of the distance, the distance divided by the period will give the amount 
of gravity expended during the period. Thus, by the two hypotheses, the amount of gravity expended during the period of a planet, will be reciprocals of each other; thus, in one case, the amount of gravity of $\mathrm{C}$ during its period, would be $2,-$ and by the other hypothesis, it would be .5 .

If the spaces passed over by two planets of a system are as the square roots of the distances of the planets, each planet will have received an equal amount of gravity with the other. So the gravity exerted on A in time 1 , is equal to that exerted on D in time $60,-$ or on $\mathrm{C}$ in time 4.

If gravity be inversely as the distance, twice the motion will balance four fold the gravity.

If gravity be inversely as the square of the distance, twice the motion will balance sixteen fold the gravity.

Upon the hypothesis that gravity is inversely as the square of the distance; and also, that twice the motion balances quadruple the force, it follows as a corollary, that if gravity were to be increased so as to become inversely as the distance, the motions of the planets throughout a system, would be equal one with another, viz., the motion of $\mathrm{C}$ would, with but 1-4th the rate of gravity, be equal to that of $A$.

But the hypothesis that gravity is inversely as the square of the distance, presents in its consideration strange dilemmas, which I will not stop here to recount. Suffice it to say that the advocates of such hypothesis claim for it, that twice the motion of a planet balances quadruple the force; when nothing is more obvious than that the terms of the hypothesis require sixteen fold the force to balance twice the motion.

But notwithstanding the hypothesis, that gravity is inversely as the square of the distance, is so generally accepted and received, astronomers have brought strong reasons to bear against it ; as in case of Jupiter and his satellites, when compared with the earth and moon; for notwithstanding those unphilosophical notions in respect to the different densities of the planets, they have gone upon the principle that twice the motion requires quadruple the force; and consequently, that gravity is inversely as the distance; and hence, that the mean motion is always the square root of the mean gravity.

From a consideration of the times of the periods of Jupiter's satellites, and of the moon, astronomers have estimated the attracting power of Jupiter to be something over three hundred times as great as that of the earth. We will suppose then, for the sake of round numbers, that the attracting power of the earth compared with that of Jupiter, is as 1 to 324 ; that the distance of our moon is 1, and consequently each of its elements 1 ; and that Jupiter's first satellite is at the same distance from 
Jupiter's centre, that the moon is from the centre of the earth. Now in such case, all astronomical calculations have gone upon the ground, that the period of our moon would be eighteen times the length of the period of Jupiter, viz., that the rate of motion in either case is the square root of the force; and consequently, that the force is inversely as the distance.

Thus the world, in relation to the law of gravity, seem to have adopted an article of faith which they have not been able, in a single instance, to practise upon. Nevertheless, the most crude and palpable error of Sir Isaac Newton, is yet held more sacred than that infinity of simple truths which are continually urging us to reject it.

\section{SECTION SIXTH.}

The attempt of astronomers, to account for all the smaller motions of the planets, of the moon, \&c., - as the aphelia, the nodes, the precession of the equinoxes, \&c., upon the principle of disturbing forces caused by the mutual attraction of those bodies upon each other, - upon the Newtonian theory of gravity, and upon the principle upon which Clairaut is supposed to have accounted for the motion of the moon's apogee, goes upon the hypothesis that matter is alike endued with an innate principle of gravity or attraction, which operates on all other matter with a force or power of attraction in an inverse ratio to the square of the distance; and hence the Newtonian theory of gravity, has, through much curious contrivance, been converted into a system of mutual disturbing forces. But if the foundation upon which the superstructure is raised be unsound, the superstructure, of course, must fall.

But it appears to me that much of the reasoning in support of both the Newtonian theory and law of gravity, as applied to what they are pleased to call physical astronomy, is somewhat a priori; as having discovered, for instance, the amount of the motions of the aphelia of the planets, and having assigned the cause to disturbing forces upon each other, it became necessary then, in accordance with their theory and law of gravity, to assign to each planet its proper share of the disturbing force, although it has not been as easy to ascertain the disturbing powers arising from what has been conceived to be the different densities of those planets which have no satellites, as of those which have ; for La Lande and La Grange differ widely as to the density of Venus, - La Grange assigning its density in proportion to its distance from the sun, as he does also that of all the other planets, and he avows that he saw no cause for assigning their densities otherwise. 
But I will quote a little from Mr. Vince, who has given a general digest of this theory, with its reduction and equation to a supposed coincidence with the facts, as found from observation. He says: "Sir Isaac Newton was the first who accounted for this motion (the precession of the equinoxes.) Having proved that from the centrifugal force of the parts of the earth arising from its rotation, the equatorial diameter must be greater than the polar, he proceeded to show, that if we conceive a sphere to be inscribed in the earth, the attraction of the sun and moon upon the excess of the quantity of matter in the earth above that of the sphere, will cause a motion in the plane of the equator, and make the points where it intersects the ecliptic go backwards upon it. But although he assigned the true cause of the precession, it is acknowledged that he fell into an error in his investigations of the effect."

This, then, is substantially the basis upon which they proceed: and after an attempted demonstration, showing that from the action of the sun alone, the annual precession would amount to $21^{\prime \prime} 6^{\prime \prime \prime}$, we find these observations, namely:

12 "This would be the precession of the equinoxes arising from the attraction of the sun, if the earth were solid, of a uniform density, and the ratio of the diameter as 229 to 230 ; but from what follows, if the greatest nutation of the earth's axis be rightly ascertained, the precession is only $14^{\prime \prime} 5^{\prime \prime \prime}$; which difference between the theory and what is deduced from observation, must arise either from the fluidity of the earth's surface, or increase of density towards the centre, or the ratio of the diameters being different from that which is here assumed; or probably from all the causes conjointly. This regression of the equinoxes (caused by the plane of the equator moving backwards upon the ecliptic) must necessarily cause the poles of the earth to describe circles about the poles of the ecliptic, in a direction contrary to the order of the signs, setting aside the effect of nutation."

Thus we perceive that heaven and earth may fail, but not one jot or tittle of the Newtonian law or theory of gravity must fail ; everything may be wrong but his theory and his promulgated law of gravity; when, in case of all his disturbing forces, as well as in respect to the motion of the moon's apogee (even if those motions depended upon disturbing forces) his law of gravity would give but one-fourth part force enough to produce the effect.

But in respect to the precession of the equinoxes, we find another motion, namely, a nutation of the poles of the earth, which is brought, in connection with the precession. to thwart and perplex the theory, and cause a division of the equation designed for the precession. 
And hence we find Mr. Vince, under the head of "Precession and Nutation arising from the Action of the Monn," giving a dissertation, mixed with commentaries from Dr. Bradley, upon his observations upon the star Draconis and others, in which reference is made to his discovery of the aberration and progressive motion of light, and in which it is said that Dr. Bradley was the discoverer of the inequalities of the precession of the equinoxes, and the nutation of the earth's axis, arising from the attraction of the moon in different situations of its nodes ; and from the length to which the dissertation is prolonged, I must refer the reader mainly to the article itself.

Nevertheless, we find, toward the close of the article, this passage: "The surprising agreement, therefore, in so long a series of observations, taken in all the various seasons of the year, as well as in the different positions of the moon's nodes, seems to be a sufficient proof of the truth both of this hypothesis and also of that which $\mathrm{I}$ formerly advanced, relating to the aberration of light." And then follows: "The conclusion derived from these observations is, that the gradual diminution of the obliquity of the ecliptic to the equator, does not arise from an alteration in the position of the earth's axis, but from some alteration in the ecliptic itself; because the stars at the end of the period of the moon's node, appeared in the same places with respect to the equator, as they ought to have done, if the earth's axis had retained the same inclination to an invariable plane," and finally the whole nutation is fixed at $19^{\prime \prime}$. And again, Mr. Vince says: "If the annual precession arising from the sun be taken at $21^{\prime \prime} 6^{\prime \prime \prime}$, as in Article 1022 , and the whole precession be $50^{\prime \prime}$, then the part arising from the action of the moon will be $28^{\prime \prime}$ 45 $5^{\prime \prime \prime}$, hence (Article 1034) the density of the moon to the density of the sun is as $28^{\prime \prime} 54^{\prime \prime \prime}$, to $21^{\prime \prime} 6^{\prime \prime \prime}$ multiplied by .988 , equal to $20^{\prime \prime} 8^{\prime \prime \prime}$, which ratio does not agree either with the proportion deduced from the tides, or with the accurate observations of Dr. Bradley. The best method of settling this point is from the greatest nutation." And finally we find the point settled, and equated, so as to make that part of the precession arising from the action of the moon, amount to $35^{\prime \prime}$ $39^{\prime \prime \prime}$, and that part arising from the action of the sun, 14" $36^{\prime \prime \prime}$.

And near the close of the chapter, we find, under the head of objections, which appear to be raised for the purpose of answering and allaying them, the following:

"Another difficulty that may arise is in relation to our having made the effect of the sun's force to be about one-third part less than the quantity resulting from calculations founded on hydrostatical principles and the hypothesis of an uniform density of all parts of the earth. But, that the phenomenon cannot be truly 
accounted for upon this hypothesis, appears from the concurrence of all experiments in general; for, whether we regard the mensuration of the degrees of the earth, the accurate observations of Dr. Bradley on the proportions and times of the tides, the case is the same, and requires a much less effect from the action of the sun than results from, or can consist with the said hypothesis. But if the density of the earth, instead of being uniform, is supposed to increase from the surface to the centre (as there is the greatest reason to imagine it does,) then the phenomenon may be easily made to quadrate with the principles of gravitation; and that according to innumerable suppositions respecting the law whereby the density may be conceived to increase."

Now, to me, this is a most extraordinary commentary upon the Newtonian theory and law of gravity, as it must be to any person who has not one particle of faith in either.

I have already remarked, that what are called the disturbing forces of the planets upon each other, are by astronomers placed on similar grounds to the supposed disturbing force of the sun upon the moon; and hence we find Mr. Vince saying, in treating of the motion of the moon's apogee, "The action of one planet upon another to disturb its motion about the sun, is similar to the action of the sun upon the moon, to disturb its motion about the earth. The general equation of the curve, therefore, which the disturbed planet describes, may represent that which the moon describes about the earth, and thence the irregularities of the moon's motions may be investigated."

And in accordance with the foregoing, he further says, "These fluxional equations are the same as those determined by Clairaut, Euler and Mayer, in their treatises upon the theory of the moon, the integration of which is a problem of great difficulty," \&c. And Mr. Vince, after making what he calls a near approximation to the ratio between the mean motion of the moon's apogee and the mean motion of the moon, concludes with these remarks: "Hence we may conclude that the theory of gravity is sufficient to give the true motion of the moon's apogee."

Now, it is rather a sad commentary upon the intelligence and independence of mind in enlightened communities, that it should be the boast and admiration of the multitude, who are ready to burst into pæans and songs of triumph at these supposed achievements in the science of astronomy, by those who are conceived to have so far surpassed the ken or comprehension of mortals, that very few persons upon the face of the globe can be expected to understand the cause and effect, or comprehend the modus operandi by which a knowledge of the operations have been achieved; and if there is too much arrogance on the one 
part, and too much implicit faith on the part of the multitude, such arrogance and implicit faith have doubtless, in a measure, had their origin in a kind of national pride, and received their impulse from those who were desirous to make the author of the Principia illustrious ; and that, too, from the fact or circumstance that not more than two or three of his contemporaries were able to understand it, and that more than fifty years elapsed before its great physical truth (the law of gravity according to Newton) was finally understood, acknowledged and appreciated; and only then by the ingenuity of M. Clairaut, by means of a problem which Mr. Vince often alleges to be one of extreme difficulty, and which the most astute are scarcely able to investigate and understand.

It is said that Sir Isaac Newton, in extending the consequences of the great physical truth contained in the Principia, gave the true cause of the precession of the equinoxes, namely, the attractive influence of the sun upon the surplus matter of the earth over and above its greatest inscribed sphere; although Mr. Vince says, "it is acknowledged that Sir Isaac fell into an error in his investigation of the effect ;" and that the celebrated English astronomer, Dr. Bradley, discovered a nutation of the poles of the earth caused by the attraction of the moon upon the surplus matter of the earth over and above its greatest inscribed sphere; which nutation, or motion of the poles of the earth, he conceives and defines to be performed in small elliptical orbits, whose diameters are about $18^{\prime \prime}$ and $16^{\prime \prime}$ about the actual poles, or what he calls the mean poles of the earth, or perhaps around what may properly be called the axis of the heavens.

This discovery of Dr. Bradley certainly caused much perplexity, for a time, to the science of astronomy, until it was finally settled (as it were by convention) what portion of the influence in producing the precession of the equinoxes should be assigned to the moon, and what to the sun; for as no division of influences would agree or correspond with the supposed proportional densities of the sun and moon, as determined in case of the tides; nor even with the critical observations of Dr. Bradley; together with many other difficulties that were found to exist when tested by their assumed law of gravity; it was at length found necessary to cut the matter short, by considering what might be produced by the nutation caused by the moon, and assigning the remainder to the sun. And hence some $35^{\prime \prime} 39^{\prime \prime \prime}$ of the precession was assigned to the influence of the moon, and $14^{\prime \prime} 36^{\prime \prime}$ to the 'sun; although some give only $30^{\prime \prime}$ to the moon, and the remainder to the sun.

Now the division of the spoil in respect to the small motions 
of the nutation of the poles, and the precession of the equinoxes, which has been portioned out to the sun and moon, and to certain great astronomers, seems to have arisen from Dr. Bradley's critical and careful observations upon the apparent motions of the fixed stars; and in particular upon the star Draconis; from which phenomena observed, he made liberal deductions as to causes and consequences; as a nutation of the poles of the earth, and a division of the influence of the sun and moon in producing the precession of the equinoxes; as also a detection of the progressive motion of light in time, its rapidity \&c., all of which deductions still pervade, our works on philosophy in liberal treatises, embellished by diagrams, and what is termed the higher mathematics. And inasmuch as we all - draw liberally upon the Dr.'s detailed observations in making deductions as to the physical causes of the phenomena observed, it is but just that the leading features in his account of them should be liberally quoted, with the view that all may compare the phenomena observed with the various deductions and hypotheses drawn from them as their physical cause.

In respect to the Dr.'s hypothesis of the progressive motion of light in time, I certainly believe it to be a very vague conjecture, which should never have been incorporated into philosophy as a component part, clothed with all the dazzling brilliancy of the higher branches of mathematics, and consequently, placed where few would conceive themselves capable of investigating the truth or error of the hypothesis.

If, however, the detailed observations of Dr. Bradley, on the star Draconis, were alone to be considered, perhaps no phenomenon furnishes more conclusive evidence of any fact connected with it, than that observed by Dr. Bradley in favor of the hypothesis that light possesses no progressive motion. But in respect to the nutation of the poles, it is believed that the Dr. was not remiss in his attempt to assign the physical cause; and it is also believed, that he is the only one who ever made a successful attempt to take from Sir Isaac Newton any thing which he had found; nor is this robbery; it is but a division of the spoil, - leaving to the lion his share, viz: his theory and law of gravity; he only took from the sun, and gave to the moon; which was certainly in accordance with the Newtonian theory. But why, after the Dr. had so fully assigned what he and everybody else then believed to be the physical cause of the phenomena, it should afterwards have been alleged, that the Dr. was the first to discover by observation, the apparent change in the declination of the stars, (but that the physical causes were afterwards explained by ' $D$ 'Alembert and others,) I know not, unless it was, that they 
discovered a discrepancy in the Dr.'s deduction of the progressive motion of light, from a supposition; that the apparent declination of the stars was about twice the amount which would be assigned to them, either in a consideration of the precession of the equinoxes, or in a nutation of the poles; for surely the Dr. in his speculations on the subject, transports us through a whole period of the moon's nodes; at the end of which he again finds things as they were, except that the equinoxes have continued their regressive motion.

There has been no disposition, however, in any of these astronomers, to break loose from the Newtonian theory or law of gravity; and inasmuch as Sir Isaac Newton, in extending the consequences of the great physical truth of the Principia, assigned the phenomena of the precession of the equinoxes, to the direct agency of gravity upon the surplus matter about the equator, over and above the greatest inscribed sphere of the earth, - of course the same has never been questioned or doubted; and hence, as those motions of the earth were placed directly under the Newtonian theory and law of gravity, and inasmuch as a law would be useless, unless it were obeyed, it is said, that D'Alembert and Laplace have succeeded by analysis in reducing all these intricate phenomena to the law of gravity with the most complete success; and such is generally understood to be the case. And inasmuch as the labor and ingenuity has been so vast in reducing, adjusting and equating them to the Newtonian law of gravity, - compared with what it would have been to have simply accounted for them upon some true or rational hypothesis, - it might seem a pity to attempt to disturb the decision, even upon the discovery of ample and satisfactory evidence.

Nevertheless, I am bound to consider that those who have reconciled these phenomena to the Newtonian law, have no more abided by the two maxims laid down by Newton, than Sir Isaac himself did; namely, "No more causes are to be admitted than are sufficient to explain the phenomena,"- and that of "effects of the same kind, the same causes are to be assigned, so far as it can be done."

Now aside from the question whether the rate or intensity of gravity be inversely as the distance, or inversely as the square of the distance, ean it be rationally conceived, or even pretended, that the position and motion of the earth requisite to produce the precession of the equinoxes, or the nutation of the poles, are any more dependent upon the direct operation, or force of gravity, than is the position of the poles or equator of the earth, in respect to the ecliptic, or the rotatory or diurnal motion of the earth upon its axis? 
And if not, then there is no direct physical necessity, that the force of gravity should be the direct or immediate cause, and hence it should not be assigned as the immediate cause, unless such shall be the necessity of the ease. "That is, if the phenomena may take place or exist without being directly or immediately caused by gravity, we then have no business to assign gravity as the direct cause. We then may draw something from analogy in sustaining the hypothesis, that the earth may have the requisite motions for producing the phenomena under consideration, without resorting to the immediate agency of gravitation; or without resorting to the ridiculous idea of a projection of the planets in tangents to their orbits in the outset of their career.

Now the nutation of the poles is called, and conceived to be, Lunar Nutation; and it is said that Physical Astronomy has made known the existence of another nutation, called Solar Nutation; but that it is too small to be detected by observation. Thus what is termed Physical Astronomy, based upon the Newtonian theory and law of gravity, has already subjected the moon to some thirty equations for the correction of supposed perturbations; and the earth seems destined to be hunted down in much the same way, unless the world should happen to discover that the attractive influence of the sun at his distance, is capable of producing more than two hundred times the motion of the earth; that the attractive influence of the moon is, at her distance from the earth.

Nevertheless, Precession and Nutation, are actual motion, and not merely position, as the obliquity of the ecliptic to the equator, or to the poles of the earth would be, were it not for the precession and nutation; and as my theory is, that all the motions of the heavenly bodies are caused and controlled (either directly or indirectly) by force, or gravity; that they are the effects of the operation of force, and not of time; hence, precession and nutation will fall within the same category as that of any other motion of the heavenly bodies, and be subject to the same laws.

\section{SECTION SEVENTH.}

It is not my design to give a theory of the moon's motions, but to endeavor to relieve her from some of those perturbations which have been caused by the attempts of astronomers and mathematicians, to bring their theory to agree with observation; and for authority on this subject, I shall mostly refer to Vince's Astronomy, vol. 2, chap. 32nd, in which the theory of the moon, as now understood, seems to have been pretty thoroughly elaborated. 
Mr. Vince, after some preliminary remarks on the subject, commences thus: "As the attractive force varies inversely as the square of the distance," \&c.

Again, Mr. Vince says, "Sir Isaac Newton, having found that the moon was retained in its orbit by a force, which at different distances from the earth, varied inversely as the square of the distance; and concluding from analogy, that the same law of attraction might take place between all the bodies in the system, applied this theory, (called the theory of gravity,) to compute the effect of the sun's attraction upon the earth and moon, so far as it might affect the relative situation of the latter as seen from the former; and hence he discovered, besides the irregularities before observed, other small irregularities of the moon's motion, which also were found to agree with observations. From these and other applications of his theory, he was confirmed in his conjectures concerning the principle of universal gravitation; and the same principle having since been further applied, and found to produce conclusions conformable to observation, his theory of gravity is now firmly established." To which is appended the following note, as farther explanation :"Sir Isaac Newton found that if the force with which bodies fall upon the earth's surface were extended to the moon, and to vary inversely as the square of the distance from the centre of the earth, it would in one minute draw the moon through a space which is equal to the versed sine of the arc which the moon describes in a minute. He concluded, therefore, that the moon was retained in its orbit by the same force as that by which bodies are attracted upon the earth."

And the truth in respect to the foregoing proposition is certainly a desideratum; and should there be error, its detection may serve to show how prone we are to receive those things for truths, at the hands or suggestions of others, which it is our business and our privilege, to examine for ourselves; and that we ought not too implicitly to rely upon the truth of those things. which others profess to have found, (however renowned for their wisdom,) lest we imbibe error in place of some great leading truth, and then call upon heaven and earth to yield their laws to sustain and corroborate it.

I will now, in short, state the premises upon which I propose to compare my result in respect to the law of gravity with that of Sir Isaac Newton's, as deduced from the law of falling bodies near the earth, and its identity with that of gravity which retains the moon in her orbit.

1. For the purposes of calculation, Newton, Maclaurin and others have gone upon the ground that the moon is sixty times as 
far from the centre of the earth as it is from the surface of the earth to its centre.

2. By the law of falling bodies, it is found that a body falling from a state of rest near the surface of the earth will fall in one second of time from its commencement, about sixteen feet, which may, for the sake of ease in calculating, be called one rod, or abstractly, one space; and the second of time in which it is falling, may, abstractly, be called one moment.

3. We will also consent, (for the present purpose,) that Sir Jsaac Newton found, from observation and calculation upon the orbit of the moon, and her motion in such orbit, that the versed sine of the arc of the moon's orbit described in 60 seconds of time by the moon while revolving in her orbit, is just one rod, or one space, or just as far as a body will fall from a state of rest near the surface of the earth in one second of time, or that the moon, at the distance, 60 from the centre of the earth, will fall just as far in 60 seconds or moments of time, as a body at the surface of the earth, or at 1-60th part of the distance of the moon from the centre of the earth, will in one second or moment of time.

Now these are the premises; and the question to be determined is, whether, to produce these results, the force of gravity towards the earth (or centre of the earth) be inversely as the square of the distance, as taught by Sir Isaac Newton, or simply inversely as the distance, as I allege it to be.

These are the premises from which Sir Isaac Newton and others have drawn the conclusion that the force of gravity varies inversely as the square of the distance varies. And these are the premises from which I draw the conclusion that the force of gravity varies inversely as the distance varies.

I contend that Sir Isaac Newton applied the inverse of the square of the distance to the intensity or rate of the force applied; when it only refers to the amount of the fall in given time; that is, in definite moments of time, to which it does apply.

For the purpose of making a few additional commentaries on the subject, I will here again quote Mr. Maclaurin's exposition of Sir Isaac Newton's hypothesis, in respect to the law of gravity, in view of the circumstance that he was a mathematician of celebrity, and also a personal friend of Sir Isaac Newton. He says, "The computation may be made after this manner: the mean distance of the moon from the earth being 60 times the distance of heavy bodies at the surface of the earth from its centre, (a body at the surface of the earth will fall $15 \frac{1}{12}$ Parisian feet in a second,) and her gravity increasing in proportion as the square of 
the distance from the centre of the earth decreases, her gravity would be $60 \times 60$ times greater near the surface of the earth than at her mean distance, and therefore would carry her through $60 \times 60 \times 15_{\frac{1}{12}}^{\frac{1}{2}}$ Parisian feet in a minute near the surface; but the same power would carry her through $60 \times 60$ times less space in a second than in a minute, by what has been often observed of the descent of heavy bodies; 'and therefore the moon, in a second of time, would fall, by her gravity, near the surface of the earth, $15 \frac{1}{12}$ Parisian feet; which therefore is the same with the gravity of terrestrial bodies."

Now this might be well enough for an article of faith, if a person had no' particular regard for its being true. But the method of begging the question, or assuming without proof, in matters that should be demonstrated, may not always make the evidence as conclusive as it may be specious.

Mr. Maclaurin says, "a body near the surface of the earth will fall $15_{\frac{1}{12}}$ Parisian feet in a second; and her (the moon's) gravity increasing in proportion as the square of the distance from the centre of the earth decreases, her gravity would be 60 $\times 60$ greater near the surface of the earth than at her mean distance ;" (that is, in case that gravity increases as the square of the distance decreases, the gravity of the moon would be 3600 times as great at the surface of the earth as at her mean distance; which would be a fair corollary or conclusion, if the premises were true;) and therefore" (Mr. Maclaurin says) would carry her through $60 \times 60 \times 15_{12}^{\frac{1}{2}}$ Parisian feet in a minute (or 60 seconds) at the surface;" - thus making the force of gravity, or urging force, increase with the acceleration, during the fall."'

Mr. Maclaurin proceeds thus: "But the same power would carry the moon $60 \times 60$ times less space in a second than in a minute by what has been often observed of the descent of heavy bodies ;" (which allegation is true, as found from observation and experience, whatever be the law of gravity;) "and therefore" (Mr. Maclaurin says) the moon in a second of time would fall by her gravity near the surface of the earth $15_{\frac{1}{12}}$ Parisian feet; wich therefore is the same with the gravity of terrestrial bodies."

And this last allegation is true, for the simple reason, that a body falling freely from a state of rest, near the surface of the earth, will fall $15_{\frac{1}{12}}$ Parisian feet in one second of time.

But why the word therefore, should have been called in aid, I know not, - as the allegation, that the moon would fall $15_{12}^{\frac{1}{12}} \mathrm{~Pa}$ risian feet in one second of time near the surface of the earth, certainly is not a corollary or consequent flowing from his first allegation, viz: that gravity increases inversely as the square of the distance decreases; as his last allegation would be true by 
any law of gravity. Neither does it apply to his second allegation, as that also would be true by any law of gravity; for it is an established fact, that necessarily applies to, and is controlled by the law of gravity, whatever that law may be.

The whole of Mr. Maclaurin's or Sir Isaac Newton's supposed demonstration, or explanation of the law of gravity, as conceived to have been deduced from the law of falling bodies, then vanishes in thin air. They have only seduced themselves by a shadow of demonstration, where no substance exists.

They have laid no premises from which conclusions are drawn, save the assumed or unproved and erroneous allegation, that the force of gravity is inversely as the square of the distance; and yet, upon those assumed premises, rests most of the fabric of modern astronomy.

But what the world would be glad to know, is, whether the force of gravity decreases as the distance increases, or as the square of the distance increases ; and upon this point, Mr. Maclaurin has given us no proof or satisfaction whatever.

And I will here protest against this hap-hazard manner of dispensing science to the world; - either in respect to the quadrature of the circle, or the law of gravitation, - wherein no premises are laid from which logical deductions can be drawn, although conclusions are made and sent forth to the world, which too often stifle further inquiry, from an implicit faith in those who have assumed to make them. But I deny to the Pope himself, the right thus to sell indulgences to the world.

Mr. Maclaurin has, however, well said, "that the moon would fall through 3600 times less space in one second, than in one minute, (or 60 seconds, ) by what has often been observed of the descent of falling bodies;" - thus consenting to the law of falling bodies as it is; and because such is the law of falling bodies the force of gravity varies inversely as the distance varies.

A falling body then, urged by a constant and uniform force through 60 seconds of time, from a state of rest, will fall 3600 times as far as in the first second of its fall. Hence if a body at the surface of the earth fall over one rod, or 198 inches, in the first moment (or second) of its fall, the moon will fall over a little more than 1-20th of an inch in the first moment or second of its fall.

Thus, if both bodies commence their fall at the same time, that at the surface of the earth will fall 3600 times as fast, at any given instant, as that at the distance of the moon will. So if the time of the fall of the moon be 60 seconds, it will fall 119 times as far in the last second or moment, as in the first, and with the same amount of force expended; and the same ratio will hold as to the fall of the body near the earth. 
Thus we see that time only compensates for the lack of the intensity or rate of force, just in accordance with the laws of dynamics, from the days of Archimedes to the present; but does not supply the place of force, or compensate for it, in any other way than that of a lack or want of intensity.

Thus Archimedes could move the world with his lever, if he had a proper fulcrum and a place to stand on; and the allegation is true in theory. But we learn from his notions of the operations of time, force, and motion, that he would expect no assistance from time; otherwise than that, if he would take time enough for the accomplishment of his enterprize, it would compensate for his weakness.

This principle in dynamics comes also under the most common observation; as in the common use of the lever in raising weights by a windlass, whether with a horizontal or upright shaft; as well as in the use of all the mechanical powers, in which we make use of time to compensate for the lack or want of the intensity of force.

Nevertheless, the same amount of force is necessary to accomplish the object, whether the time be longer or shorter; for time does not do the work, but only furnishes the time, and hence does not compensate otherwise than for a lack of power to do it sooner.

Hence there will be just as much force of gravity expended in 60 seconds at the distance of the moon, as in one second at the surface of the earth; and in either of the 60 seconds at the distance of the moon, there will be 1-60th part as much force expended, as in one second at the earth's surface.

But if the force of gravity be inversely as the square of the distance, I believe all will readily agree, that the whole amount of force expended on the moon in sixty seconds of time, will be but the 1-60th part as much as that expended on the body near the earth in one second of time; in which case, at the distance of the moon, time must actually supply the place of 59.60ths of the force requisite to urge it over the given space, unless we adopt another hypothesis, equally though no more absurd, namely, by supposing the force to increase as the motion accelerates, or as the odd numbers in their order; in which case, 119 times the force that is expended in the first would be expended in the last second of time.

For no one will contend, if the force of gravity be inversely as the square of the distance, that more than 1-60th part of the force is expended on the moon that is expended on the body near the earth while passing over equal space.

But if the force of gravity be inversely as the distance, the 
same amount of gravity or force will be expended on the moon while falling over a given space, as will be expended on a body near the earth while falling over an equal space, but in 1-60th part of the time; in which case the lack or want of intensity of force at the distance of the moon, would be compensated for by timé, in accordance with all the laws of dynamics with which we are acquainted.

Hence we arrive at this important fact in the law of falling bodies, namely, if we conceive an individual body to fall over a given space, and that the time of its fall be divided into any number of equal moments, we must also conceive the amount of force applied in each moment, to be equal one with another; then, in the first moment, if the amount of space fallen over be called 1, the amount fallen over in the second moment will be 3 , only the like amount of time or force being expended as in the first moment; in the third moment, the spaces will be 5 , and so on, by the odd numbers; in which case, at the end of any given moment, the whole number of spaces fallen over will be as well the square of the several amounts of force expended in all the several moments, as of the sum of the moments themselves.

But if we compare two bodies commencing their fall at the same time towards the gravitating or attracting power, at different distances from such power, as in case of the moon and a body near the surface of the earth, namely, one body at the distance 1, and another body at the distance 60 , for example, we shall have the following results, namely: It is manifest that if we conceive the two bodies, one near the surface of the earth, at the distance 1 , and the moon at the distance 60 , to have fallen over an equal space, namely, one rod for example; that near the earth in one second, and the moon in 60 seconds of time; then, taking the seconds as the time, the times of each, in falling over equal space, will be as their respective distances from the centre of gravity; while the force employed on each will be as the amount of space fallen over. Hence, in either case, the amount of space fallen over or described, will be the square of the whole amount of force employed in the fall; while the amount of force employed upon the moon during its fall, is but.1-60th part of the time of its fall.

Hence, the Newtonian law of gravity essays to convert time into actual force, in lieu of permitting it to perform its legitimate office of compensating for want of intensity of force, which has been well known to be its legitimate office for thousands of years.

Thus have I endeavored fairly to present the evidence, the materials, the elements, from which Sir Isaac Newton deduced his 
conclusion that the force of gravity is inversely as the square of the distance, and from which materials or elements I deduce my conclusion that the force of gravity is inversely as the distance.

And hence, as a corollary or consequent of my determination, if the mean motion of a planet (whether revolving in a centric or eccentric orbit) were to be doubled in order to retain it in its orbit, the mean. force would only have to be quadrupled, or increased four-fold, in lieu of being increased sixteen-fold, as would be requisite by the Newtonian law of gravity; and consequently, the motion of the moon, whether in its simple revolution about the earth, or in the motion of its apogee, (as well as the motion of each heavenly body,) would be entitled to just four times as much instant or constant force by my determination of the law of gravity, as by that of Sir Isaac Newton, as will be readily perceived by recurring to a system of planets which revolve about one and the same central power, as in case of the planets about the sun.

And now, - notwithstanding my belief is that $I$ have, in the most direct and conclusive manner, confuted the Newtonian determination in respect to the law of gravity, by showing that he assigned the square of the distance to the intensity of the force, when it should have been assigned to the amount of the fall, (or to what they have seen proper to call the amount of the deflection of the planet from a tangent to its orbit,) or the result of the force applied, - I will, by way of reductio ad absurdam, present some few of the difficulties and perplexities under which the philosophers, the astronomers, and the mathematicians of Europe for a long time labored, in their endeavors to reconcile the Newtonian law of gravity with observed phenomena, and the facts that were found from observation to exist; and in which what they supposed themselves required to adopt as a first principle, namely, the Newtonian law of gravity, led to so much confusion and mystery, as to cause them for a long time to conspire and rebel against the law so promulgated; until at length, in despair of extricating themselves from its unjust requirements, they assented to and ratified the treaty so ingeniously devised by the great French mathematician Clairaut.

And here I will render the only rational excuse which I can devise in justification of their final acquiescence in, and acknowledgment of the Newtonian law ; namely, that they could not conceive an error to have been made in respect to the well known law of falling bodies; and hence, they even considered it useless, as well as derogatory to Newton, to investigate what he had determined as being the result of so simple and easy a calculation. 
But this is a subject which I propose to press upon the world; - to ask the institutions of my own, and of foreign countries, to reinvestigate ; and that I shall, eventually, have supporters, who will dare to risk their reputation by venturing to think for themselves, I have no doubt.

But to refer to some of the difficulties under which the mathematicians and astronomers of Europe have labored, in respect to the law of gravity, as promulgated by Newton, I quote from Mr. Vince, who though a very honest recorder of scientific discoveries, is nevertheless, quite partial to Sir Isaac Newton.

Mr. Vince, in the introductory remarks to his treatise upon the Theory of the Moon, says, "To enter into a computation of all the effects of the disturbing force of the sun upon the moon, and their nature, would require the investigation of the nature of a curve described by a body attracted to two points, called the Problem of three Bodies ;" which problem, he suggests, has been solved by but very few mathematicians, amongst whom are Clairaut and some few others. And it appears, that for more than half a century from and after the time that Sir Isaac Newton promulgated his theory and law of gravity, the learned world essayed to apply them to the motions of the moon. Mathematicians were exceedingly perplexed with Sir Isaac Newton's law of gravity; for Mr. Vince proceeds thus: "In the year 1747, M. Clairaut, in a memoir read before the Academy of Sciences, in Paris, made an objection to this law, upon this ground; that it would not account for the motion of the moon's apogee, it giving, according to his calculations, that motion only one half of what it was found to be by observation; and he concluded, that it was necessary to change this law, by adding something to correct it. He however soon after discovered his mistake, and was the first who gave a complete theory of the moon, in which he showed that Sir Isaac Newton's law of gravity would not only account for the motion of the moon's apogee, but also for all the irregularities of the moon," and Mr. Vince proceeds to say, that, "M. Euler has done great justice to M. Clairaut upon his solution of this important problem, in a letter to Rev. Casper Witstien; in which he observes, that, 'This question is of the last importance; and I must own, that till now, I always believed that this theory did not agree with the motion of the apogee of the moon.' M. Clairaut was of the same opinion; but he has publicly retracted it, by declaring that the motion of the apogee is not contrary to the Newtonian theory. Upon this occasion, I have renewed my inquiries on this affair; and after most tedious calculations, I have 
at length found to my satisfaction, that $M$. Clairaut was in the right, and that this theory is entirely sufficient to explain the motion of the apogee of the moon. As this inquiry is of the greatest difficulty, and those who hitherto pretended to have proved this nice agreement of the theory with the truth, have been much deceived, it is to $M$. Clairaut that we are obliged for this important discovery, which gives quite a new lustre to the theory of the great Newton; and it is but now, that we can expect good astronomical tables of the moon."

And in accordance with the foregoing suggestions of M. Euler, we find from Mr. Vince that various had been the attempts to reconcile the Newtonian law of gravity with fact and observation in respect to the motion of the moon's apogee; for Mr. Vince says that "M. Walmsley, in his theory of the Motion of the Apsides, has computed the mean motion of the moon's apogee, and his conclusion agrees very well with observation; but his principles are altogether wrong; for he has entirely omitted that part of the force which acts in a direction perpendicular to the radius, which, as we have shown, produces just one half of the motion; he also assumes the mean disturbing force in the direction of the radius as acting constantly, instead of the real disturbing force; and he also wrongly computed the periodic time of the moon; it was by accident therefore that he obtained the mean motion; in respect to the true motion his conclusions are erroneous; he says Mr. Machin has not given us his process; we cannot therefore say how far it was just. He says also, in the Phil. Trans. of 1751, that Mr. P. Murdock has given a method of computing the mean motion of the moon's apogee, by first considering only that part of the disturbing force which acts in the direction of the radius; and then instead of supposing the earth to be at rest, by conceiving the earth and moon to revolve about their common centre of gravity, he imputes about one half of the motion to that cause, and thence deduced a conclusion agreeing with observation.

"What we have already observed (Art. 861) is sufficient to show, that no part of the effect can arise from the latter circumstance ; and he has also (as we have already shown) omitted a cause which produces about one half the motion; by two mistakes he-has therefore fallen upon a true conclusion." And Mr. Vince, in conclusion, makes these remarks: "but in whatever manner this subject is treated, some corrections are applied from observations; in order to render the equations more perfect; not that the principle of attraction is insufficient to furnish conclusions which shall agree with observation," \&c. And again: "Thus we have given the reader all the satisfaction we 
are able upon this difficult subject, without entering into a direct solution of the problem, which requires the integration of a - fluxional equation of the second order, and this can be done only by an approximation of a very intricate nature, and of great labor."

The reader or inquirer who will take the trouble to examine the moon's motions, and especially the motion of its apogee, as contained in Mr. Vince's Astronomy, will find that in all cases, the little motions and inequalities of motion, are, in whole or in part, assigned to the disturbing force of the sun upon the moon; and after much laboring of the author on the subject, we find this allegation, namely: "If the force perpendicular to the radius vector be neglected, the motion of the apsides comes out one half of what is here determined, and this we know from other principles. This, therefore, tends to confirm the legality of the method here employed," - which allegation, after all, I think, must be taken for granted, rather than as being proved; and in respect to the whole matter, I have come to the following doubts :

I think it doubtful, whether the student or inquirer, on a careful examination of the whole subject, will readily agree with the modus operandi by which the observed phenomena of nature have been supposed to be reconciled with the Newtonian law.

Whether he will find the ratio between the force applied to the moon, and the moon's motion in its orbit, clearly and distinctly assigned or stated.

Whether he will clearly discover how they have obtained the force which acts perpendicular to the radius vector, which is said to give just half the motion of the apogee.

Whether he will be able to discover by what law of gravity, the distance may be assumed at unity, and its mean force of gravity at less than unity; or in other words, whether according to $\mathrm{Mr}$. Vince, the mean force of the moon towards the earth is equal to the natural gravity of the moon towards the earth, diminished by the disturbing force of the sun upon the moon in the line of the radius vector,- together with many other matters equally dubious, which the inquirer is required to pass through, in order that he may be brought to render assent to their results, or rather to their conclusions.

And finally, I am doubtful whether the inquirer will readily discover, that even Sir Isaac Newton understood the subject in the same way and manner in which it is now supposed to be understood, notwithstanding Mr. Vince's supposition of what Newton must have meant by' some very obcure passages in his Principia ; and more especially, when it is acknowledged by 
Mr. Vince, that Sir Isaac Newton left out the force which is now said to act perpendicularly to the radius vector; and which is said to give just one half of the motion to the apogee.

Nevertheless, Walmsley, Murdock, and others, have been somewhat cavalierly treated, for their attempts to make the Newtonian law of gravity, account for the phenomena of nature, which, manifestly, are the result of some kind of law of gravity; notwithstanding, they made their conclusions agree very well with observation. And I must say, that I think the treatment they received from the inquisition - from those who would settle the controversy by establishing articles of implicit faith, however my terious - was somewhat harsh and arbitrary.

Walmsley and others, made use of all the force that was allowed them by the Newtonian law of gravity; and as to the remainder that was found necessary for the purposes intended, each and every one, (not even excepting Clairaut himself,) picked it up from such sources as were in his view most available; and I have yet to learn why others; (as well as Clairaut,) had not the right to call to their aid, for the purpose of triumphing over great difficulties, such auxiliary forces as they could best command.

But wisdom would have been profitable to direct; and had those great mathematicians, in lieu of confiding in their high-priest, just spent an hour or two in examining, to see from whence he derived his authority, they would not have been thrown into all these inexplicable dilemmas. They would have found just force enough to account for all the observed phenomena, without resorting to anything factitious or unnatural.

Nevertheless, however gloomy the stubborn fact may be, if Sir Isaac Newton's law of gravity be erroneous, it wholly destroys his whole system of philosophy; and in such case, his theory of universal gravity, (as by him promulgated,) ceases to exist ; and the universe will again be governed by immutable and controlling laws, in lieu of depending on fortuity or chance. 


\section{CHAPTER III.}

Of the Proper Elements from which to determine the Laws of Force and Motion, incident to the Heavenly Bodies in their Eternal Rounds.

\section{SECTION FIRST.}

$\mathrm{H}_{\mathrm{AvING}}$ attempted to show that what astronomers have called the deflection of a planet, or the deflection of a planet from a tangent to its orbit, and which they have made use of as an element, in their endeavors to account for the agreement between the force and motion by which a planet revolves, is, at least, but a factitious element, and not applicable to the laws of force and motion, as operating in the curve of an orbit, nor identical in its mathematical construction with that of the law of the rectilinear fall of a body through space, - I will now proceed to treat of the proper elements of the planets from which to deduce the modus operandi by which they are retained and controlled in their orbits while performing their eternal rounds, - which elements I will call by the appellations of Time, Distance, Motion, Force, and Convergency; the qualities or principles of which I will define as follows: Time always has direct reference to the Period, namely the periodic time, or entire revolution of the planet from any given point in its orbit, to the same point again; which period or periodic time, may be divided and subdivided, as occasion may require, when comparing other elements with that of time; and hence the period or whole periodic time of a planet, as also the mean distance of a planet from the centre of gravity may, in contradistinction to the elements of Motion, Force, and Convergency, properly be called positive quantities, by which, what may be called the rates (ratios or proportions) of the elements of motion, force and convergency may be compared.

Distance, or the mean distance of a planet, may always be abstractly expressed by some numerical quantity, by which the mean distance of any given planet of a system is proportioned to the mean distance of that planet of the system which 
is assumed at unity; thus, if the mean distance of our earth from the sun be assumed at unity or 1 , the mean distance of Mercury will be expressed by the fraction .38710 ; that of Venus, by the fraction .72332; that of Mars, by unity plus the fraction .52998, \&c. So also if the orbit be eccentric, we speak of the mean distance, the perihelion distance, \&c.

The mean distance of a planet determines or denotes the circumference of the orbit, and consequently, the amount of space passed over in an entire period. It also determines the dilatation of the orbit, which is consequently as the mean distance. But the dilatation of the orbit which is denoted by the mean distance, must not be confounded with the rate of convergency of the planet, which is denoted by the time of the period. Motion, which I shall use as synonymous with Velocity, denotes a passing over space; and as this takes place in time, it also denotes the velocity or rapidity with which space is passed over.

Motion manifestly depends upon the force of gravity, at least, in retaining the planet in its orbit; and if the force of gravity be inversely as the distance, (as I contend,) the rate of mean motion will always be inversely as the whole amount of the force of gravity expended during the entire period of the planet.

Force, which I shall use as synonymous with gravity, or the force of gravity, denotes the power of attraction by which one body is drawn towards another, causing the tendency of one body towards another; as that of a planet towards the sun, or a satellite towards its primary; the intensity, or rather, proportional intensity of which manifestly depends upon the proportional distance; and it increases or decreases in the inverse ratios of some given root or power of the distance; which intensity I shall in general denominate the rate of gravity.

And as by my determination of the law of gravity, the rate of mean motion will always be found to be inversely as the whole amount of the force of gravity expended during the entire period of the planet, so also, by the same law, the rate of mean force will always be inversely as the whole amount of motion during an entire period of the planet, - thus reciprocating with each other upon perfectly equal and mutual terms.

Convergency is measured by degrees, minutes, \&c. of the circle, and hence the amount of convergency in the time of the period of any planet in $360^{\circ}$; consequently the amount of convergency in any one orbit is properly unity.

Convergency is no part of the motion of a planet; but simply the direction of a motion; nevertheless, there could be no convergency without motion; and although convergency is produc- 
ed by constant gravity, we perceive that motion is as essential to this element as gravity is. Hence, motion and convergency seem to be alike intimately connected with, and dependent upon gravity, for the exhibition of their phenomena. Nor is it certain that motion is an element that is any more independent of gravity, than convergency is, or that gravity is any less attentive to motion than it is to convergency, - notwithstanding those notions drawn from the Newtonian theory of the universal and equal gravity of matter.

But this theory of universal gravity being established, and inasmuch as gravity was to have little or nothing to do with motion, otherwise than to accelerate or retard it, it was necessary that a planet should be projected in a tangent to some point of its orbit, thereby giving motion; which, whether it were more or less, would be taken charge of by the innate gravity of distant matter. And as such force would not be likely to produce a motion just sufficient to balance the force of gravity, those elements were left to keep the balance of power, by alternately getting the better of each other; which contrivance, however, was found insufficient, until a centrifugal force was finally attached to the machinery, endued with such forces as might be found necessary to accomplish the object.

Next, the planets were found to be of different densities, in -proportion to their respective bulks or magnitudes; because it was found they did not possess the same power of attraction in proportion to their magnitudes; and notwithstanding, had analogy been applied to the train of facts which bear upon that question, such hypothesis would long since have been overthrown. But having adopted the Newtonian theory of innate and universal gravity, as the first great article of faith, neither fact nor reason has made inroads upon it.

The hypothesis of universal gravity being thus far established and put at rest beyond a wish or disposition to doubt, to ascertain the ratio which the force of gravity or attraction bears to the distance from the attracting body, became a disideratum; and M. Vince says, that Sir Isaac Newton gave Dr. Halley "a proof" of the law of gravity as proportioned to distance, namely, that it varies inversely as the square of the distance. And this hypothesis is supposed to have been clearly deduced from a comparison of the law of falling bodies near the earth, with the rate of deflection of the moon from a tangent to its orbit.

Thus, in lieu of resorting to the natural elements of the planets for the purpose of determining this very important question, they have created for the purpose, the factitious element of deflection, so wholly extraneous and foreign to the laws of force 
and motion, as to have been a very troublesome element to astronomers and mathematicians ; for having suffered their imaginations to run off in a tangent to the orbit, where no force nor motion is, (though but a very little distance,) in accordance with the precaution from the highest authority, yet if a mathematical error be contained in such very little distance, it may, nevertheless, in its consequences, be exceeding broad.

Any amount of deflection is conceived to be measured or denoted by direct linear quantity, namely, by a perpendicular line from the given tangent to the revolving body in the orbit; and hence, if the rate of progression of the supposed fall or deflection of the planet from the tangent, be ascertained or established, it could not well be made to apply to the entire period or revolution of the planet; nor could the whole amount of deflection in the time of the period be well ascertained, which should be the case with any element which may properly be said to have its rate, namely, its ratio to that of other elements of the same planet.

Nevertheless, what may be termed the element of deflection, is by no means as useless as that of a centrifugal force, but is susceptible, when rightly considered, of furnishing or throwing much light upon the law of gravity in respect to distance; and this too by comparing it with the law of falling bodies near the earth; in which comparison, we find many things in which they corroborate each other; and hence, the identity of the force which causes a body to descend or fall to the earth, and that which retains the moon in its orbit, goes in no sense to prove, that the deflection of a planet from a tangent, proceeds by the same progression as that of a falling body from a state of rest, namely, by a progression which shall be denoted by the successive odd numbers, as $1,3,5$, \&c.

For if so, then the amount of deflection of a planet whose distance is 4 , when it bas revolved over 1-8th of its orbit, should be equal to the amount of deflection of a planet of the same system whose distance is 1 , when it has revolved over 1-4th of its orbit; for the motion of the planet whose distance is 1 , must be twice as great as that whose distance is 4 , for otherwise the squares of their periods could not be as the cubes of their mean distances from the sun.

But in lieu of the rates of deflection being equal, when that whose distance is 1 , has passed over 1-4th of its orbit, and that whose distance is 4 , has passed over 1-8th of its orbit, their amounts of deflection will then be proportioned to each other, as 1 to 1.17156 , and hence the amount of deflection of a planet while revolving over 1-4th of its orbit will be 2.41421 times as great in 
the last half of the time, as in the first half; when by the law of falling bodies, it should be just three times as great in the last half of the time as in the first half.

I am aware that Sir Isaac Newtonand others have said, that if from the point of intersection, or touch of the given tangent with the orbit, we take a very little distance, the deflection will be likened to, or rather be identical in its mathematical construction with the law of falling bodies near the earth; which allegation has certainly supplied the place of mathematical certainty for a long time. But surely we ought to have been informed where the little distance should stop; for as the progress of deflection in any assignable portion of its orbit, is different from that of a falling body near the earth, it would seem necessary, in order that such little distance might present a mathematical truth, that it should be less than any assignable quantity, namely, in its ultimate ratio, alias in 0 , or no distance whatever; in which point or place, far too many important questions have been settled; as if ratios could as well exist in 0 , as in actual numerical quantities ; which, in point of demonstration, would seem too much to resemble those important discoveries we have somewhere read of, by which many of the laws of gravity and attraction were deduced and explained, by geometrizing upon two inconceivably small corpuscles, or physical atoms.

I will now return to a further consideration of convergency, and the other proper elements of the planets.

Convergency is most directly connected with the phenomena of motion in the heavenly bodies, and with the law of gravity; and being the description or delineation of the degrees, minutes, seconds, \&c. of a circle, produced in time, which becomes full and complete in the time of the period, hence, the rate of convergency is inversely as the time of the period; and the amount of convergency in the time of the period, is in all cases the same, namely, $360^{\circ}$.

Thus, while time, or rather the Period, and Distance, may be called the positive elements of the planets, those of Motion, Gravity, and Convergency, may properly be called the relative elements; performing relative operations in respect to each other, and also in respect to Period and Distance.

The relative elements are therefore properly said to have their rates of operation, not only in respect to each other, but in respect to time or period, and distance; which rates of operation become complete and entire in the time of the period, which makes what may be called the whole amount of the operation. And thus, Motion, Gravity and Convergency, are said to have both their rates and amounts of operation.

The period of a planet is the cube of the square root of the 
mean distance; so then the mean distance is the square of the cube root of the period; for if it were otherwise, the square of the period of a planet could not be the cube of the mean distance; nor could the squares of the periods of a system of planets be as the cubes of their mean distances from the sun. And that the rates, and amounts of motion, gravity, and convergency, are so proportioned to each other; and to the period and distance, as to produce that grand proportional result, is what no one will question or doubt.

Hence the rate of mean motion of a planet is inversely as the cube root of the period; or the square root of the mean distance; and the amount of motion in the time of the period, is as the mean distance; for the mean distance denotes or represents the circumference of the orbit, and consequently the amount of space passed over; which properly denotes or determines the amount of motion. Hence, the mean rate of motion is to the whole amount of motion, as unity is to the time of the period.

The rate of gravity or force, is inversely as some power or root of the distance; which I assume to be inversely as the first power, or first root, of the distance, viz., inversely as the distance. Hence, the mean rate of gravity of a planet is inversely as the mean distance, and also inversely as the square of the cube root of the period, and is consequently the square of the mean rate of motion. The whole amount of gravity, then, in the time of the period, will be the square root of the distance, which is also the cube root of the period; and hence the mean rate of gravity is to the whole amount of gravity, as unity is to the time of the period.

The mean rate of convergency is inversely as the time of the period, or inversely as the cube of the square root of the distance; and hence, is the cube of the mean rate of motion, and the cube of the square root of the mean rate of gravity or force. Consequently, the whole amount of convergency in the time of the period is unity or 1 ; and hence, the mean rate of convergency is to the whole amount, as unity is to the time of the period; or rather, as the inverse of the time of the period is to unity.

Hence, the mean rate of motion, of gravity, or of convergency, is to the whole amount in the time of the period, as unity is to the time of the period.

Hence, the product of the mean rate of motion by the time of the period, gives the whole amount of motion in the time of the period.

The product of the mean rate of gravity or force, by the time of the period, gives the whole amount of gravity or force in the time of the period. 
And the product of the mean rate of convergency by the time of the period, gives the whole amount of convergency in the time of the period.

Thus the mean rate of gravity of a planet is always a mean proportional between the mean rate of motion, and the mean rate of convergency; and is equally attentive to both. Nor does it ever forsake its position, or neglect its trust; for whether the orbit be centric or eccentric, the element of gravity at all times, and in all parts of the orbit, preserves the same position in respect to the elements of motion and convergency, viz., a mean proportional between them, and hence, the planets sing through the heavens with as much harmony and unity of purpose, as we can desire.

Kepler, in addition to the great law, "That the squares of the periods of the planets, are as the cubes of their mean distances from the sun," also discovered the important fact, "That the motion of an individual planet revolving in an eccentric orbit, varies in the inverse ratio to that of its varying distance from the sun." . But Kepler did not discover that the rates of motion of the planets are inversely as their distances from the sun; although such has often been the version and the understanding in respect to his discovery. And it requires but little consideration to convince any one, that the rates of motion of the planets are not inversely as their mean distances from the sun; for if it were so, the squares of the periods of the planets would be as the fourth powers of their mean distances from the sun; or in other words, the time of their periods would be as the squares of their mean distances from the sun ; as any one will discover on a moment's reflection. For if the mean distance were 4, and the mean motion were inversely as the mean distance, the period would be 16 , in lieu of 8 . And hence, such hypothesis is untrue.

And yet, this erroneous version of Kepler's law, has been a great supporter of the hypothesis, that the rate of gravity is inversely as the square of the distance; which error was committed by Sir Isaac Newton, in referring the square of the distance to the rate or intensity of gravity, in lieu of referring it to the rate of deflection, or to the result or effect of the gravity.

Thus, once having adopted the well established fact in dynamics, that twice the motion requires quadruple the force to retain a planet in its orbit, it necessarily follows as a corollary or consequent, that if the mean motions of the planets of a system, are inversely as their mean distances from the sun, the force of gravity which retains them in their orbits, must, of necessity, be inversely as the square of the distance from the sun. And the 
next corollary or consequent, which as necessarily follows, is, that their periods will be as the squares of their mean distances from the sun; or that the squares of their periods will be as the fourth powers of their mean distances from the sun; and also that the period of each planet of the system will be the square of its mean distance from the sun, by which, error begets error:

And the same or like consequences would follow, in a system of eccentric orbits, that would in a system of centric orbits, viz., if the mean motions of the planets were inversely as their mean distances from the sun; and also if from the points of mean motion, the motion of the planet does vary in the inverse ratio as the distance from the sun varies, as Kepler has shown; then their motions throughout their respective orbits would be inversely as their distances from the sun; presenting precisely the same or similar results in this respect, as would be presented in a system of centric orbits, viz., the period of each would be the square of the mean distance; and the periods of the respective planets of the system, would be as the squares of their mean distances from the sun; or the squares of their periods would be as the fourth powers of their mean distances from the sun. And if such were the case, the consequence would conclusively follow, that the force of gravity would be inversely as the square of the distance from the sun.

But Kepler taught no such doctrine; he did not attempt so readily to abrogate and make void his great law, so well founded in observation and in the powers and roots of numbers, viz., that the squares of the periods of a system of planets are as the cubes of their mean distances from the sun; nor to overthrow the well established fact, that twice the motion requires quadruple the force, when he thus declared the no less important discovery: That the motion of $a$ planet, viz., an individual planet, revolving in an eccentric orbit, varies from the points of mean motion, in the inverse ratio as the distance varies 'from the sun.

I say that the discovery by Kepler, - that in an eccentric orbit, the motion varies from the points of mean motion in the inverse ratio as the distance from the sun varies, - is no less important than are his other discoveries. For if it were otherwise, the revolution of a planet in an eccentric orbit would be wholly inexplicable.

It is this principle that chains the planet in its eccentric orbit, and keeps it obedient to the power of gravity, as much so in one part of the orbit as in another; and hence prevents, or avoids the fortuitous necessity, that gravity should get the better of the motion at aphelion; and that the motion should get the better of gravity at perihelion, in order to preserve the balance of power, 
by the help of a centrifugal force; as the laws of gravity and motion have been taught and explained by Maclaurin, Ferguson and others, to a world of too easy faith.

Hence, it will be found, (as I propose to show in the sequel,) that both the gravity and convergency of a planet revolving in an eccentric orbit, vary from their mean, in the same ratio that motion does, viz., in the inverse ratio as the distance varies from the mean, or from the sun; which is one and the same thing. And hence, the rate of gravity always remains a mean proportional between the rate of motion and the rate of convergency, controlling both of them, as well in one part of the orbit as in another.

But in respect to the Newtonian law of gravity, viz., that gravity is inversely as the square of the distance, nothing can be more manifest, than that, in order to sustain such law, the mean motion of any planet of a system, whether revolving in a centric or eccentric orbit, must, in reference to the mean motions of the other planets of the system, be inversely as the mean distance, which is not the case; but in such case, the squares of the periods of the planets would be as the fourth powers of their mean distances from the sun.

\section{SECTION SECOND.}

That the apparent motions of the planets are not equable in their orbits, seems to have been familiarly known to ancient astronomers; and hence, that the orbits in respect to the earth, as was then supposed, were eccentric.

This seems to have been the opinion of Ptolemy in ancient times, and of Tycho in more modern times; and upon this hypothesis, they endeavored to equate their motions; but when it became more rationally understood, that the eccentricity of the orbits referred to the sun directly, and not to the earth, the idea that each planet revolved around some point in its orbit, about which the motion was equable, was of consequence abandoned, and gave place to new researches into the phenomena of the motions of planets, preparatory to accounting for such eccentricity.

In this respect, Kepler, who always seemed preëminent in searching out physical cauess and phenomena, discovered the fact, - which is perhaps no less important in respect to eccentric orbits, than is his great law that the squares of the periods of a system of planets, are as the cubes of their mean distances from the sun, in respect to orbits in general, - that the motion of a planet revolving in an eccentric orbit, varies inversely as the 
distance from the sun varies; which law is so essential and important in respect to the motion of a planet in an eccentric orbit, that any attempt to account, without the law, or to attempt to supply its place by extraneous matter, would to me appear like a forlorn hope. And why this law, in connection with the other great law just quoted, should not long ere this have disclosed more important truths in respect to the laws of force and motion than they have done, appears to me wholly unaccountable.

It will be readily seen by any one who will examine the subject but a little, that a misapprehension of said law, viz., that the motion of a planet varies in its orbit inversely as the distance from the sun varies, would lead to, and produce the same inexplicable difficulties which now pervade the science of astronomy ; and that said law has been wholly misapprehended, there perhaps is but little doubt.

If the mean motions of a system of planets (whether composed of centric orbits, eccentric circles, or ellipses),were inversely as their mean distances from the sun, and if also in each eccentric orbit, the motion should vary from the point of mean motion inversely as the distance from the sun should vary while revolving in its orbit, in such case nothing can be made more manifest; no more simple proposition exists than that force or gravity must of necessity be inversely as the square of the distance. And it is, also, every way as simple a manifestation, that if such were the case, Kepler's other great law would be made wholly void; for in such case, the squares of the periods of a system of planets, would be as the fourth powers of their mean distance from the sun.

In such case, the mean motion of a planet whose distance is 4 , would have to be .25 , - when we know it must be .5 in order to fulfil the great law. And the only possible condition, as I have already shown, upon which the hypothesis that the force is inversely as the square of the distance, could be even hypothetically sustained, would be upon the ground, that the mean motions of a system of planets are inversely as their mean distances from the sun; and also, that the motion of any planet revolving in an eccentric orbit, varies from the point of mean motion, inversely as the distance from the sun varies.

But Kepler adopted but the last part of said hypothesis, viz., that a planet revolving in an eccentric orbit does vary from the points of mean motion inversely as the distance of the revolving body varies from the sun; and this permits his other law to stand, and all the elements of the planets to operate in unison.

The mean motions of the planets then, are inversely as the 
square roots of their mean distances from the sun; that the cubes of their mean distances may be as the squares of their periods.

But what has, perhaps, served much to sustain mankind in their misapprehension of Kepler's law, is that useless demonstration, (so far as astronomy is directly concerned or ever has been,) that if a revolving body vary in its motion inversely as its distance varies from the sun, it will describe or pass over equal areas in equal times. This demonstration, although true, has never been used in astronomy to any other effect than "to perplex and dash maturest counsels."

In any system of planets, whether all the orbits of such system are centric, eccentric, or elliptical, if their mean motions were inversely as their mean distances from the sun, the planets of such system would in respect to each other describe equal areas in equal times.

In such case, the mean motion of the planet whose mean distance js 4 , would be .25 , or half what we know to be its actual motion; and its period would be 16, or the square of its distance, or twice its actual period; and the area passed over in the time of its period, would be 16 times as great as that passed over by the planet whose distance is 1 , in the time of its period. That is, in such case, the areas passed over by the several planets of a system, would be inversely as their motions ; or would be as the square roots of their respective distances; and hence, would be equal in equal times. Then, indeed, would the force be inversely as the square of the distance; and the motion would be the fourth root of the force; the squares of the periods of a system of planets, would be as the fourth powers of their mean distances from the sun; or their periods would be as the squares of their mean distances from the sun.

If the distance of the earth from the sun were assumed at 1 , the distance of a planet whose period was 8 , would be the square root of 8 , in lieu of the square root of 16 , as now supposed. Thus, the distances of the planets of our system would be reduced from that of the third roots of the squares of their respective periods, to that of the square roots of their respective periods. The distance of Herschel from the sun would be much less than half the distance it has heretofore been estimated at, even though the earth were distant $95,000,000$ miles. But as Venus would have a greater proportional distance from the sun, in proportion to that of the earth, than has been assumed, (unless from its greatest elongation or otherwise, its true proportional distance has been determined,) hence, the actual distance of the earth from the sun in miles, would have been overrated, 
in which case the actual distance of all the superior planets would have been overrated in like proportion.

So it seems, in such case, the superior planets would, in a measure, be relieved from the charge of a lack of density in proportion to their respective bulks or magnitudes, which has been charged against them by some; as their bulks or magnitudes would be vastly less than had been supposed; with an innumerable train of consequences which I will not here attempt to follow out.

It then becomes conclusive, that if the mean motions of the several planets of a system are inversely as the mean distances from the sun, the periods of the planets will be as the squares of their mean distances from the sun.

But Kepler only intended to declare, and only did declare, that the motion of any individual planet of a system varies from the point of mean motion (if the orbit be eccentric) inversely as the distance varies from the sun: In which case the radius vector of such planet will pass over or describe equal areas in equal times; notwithstanding the mean motions of all the planets of the system are inversely as the square roots of their respective distances. And in which case also, the great law that the squares of their periods are as the cubes of their mean distances, will still apply to the system in all its force and majesty.

I shall adopt, fully, Kepler's two said laws as he gave them; and consequently adopt the hypothesis, that the force or gravity, of any individual planet, varies inversely as the distance varies from the sun; as that of necessity follows upon the adoption of said two laws. And it is only upon the adoption of those principles, that we are enabled in anywise to account for the motions of a system of planets revolving in eccentric orbits. And that the primary planets of the solar system do all revolve in eccentric orbits, is doubtless true; and that such is the case with all the heavenly bodies, is, at least, more than probable.

The adoption then of Kepler's law, that the squares of the periods of a system of planets are as the cubes of their mean distances from the sun; and also, his law which teaches that the motion of a planet revolving in an ecceniric orbit varies from the points of mean motion inversely as the distance varies from the sun in the course of its revolution; together with the necessary and imperious consequent upon said law, viz. that the force of gravity varies inversely as the distance varies from the sun,-furnishes a very easy and simple' solution, accounting for the movements of a system of planets in eccentric orbits; and we shall hence discover that those principles are entirely 
able to manage a planet throughout its entire period, without the aid of any other machinery whatever.

I will suppose a system of planets revolving around the sun, or around one and the same central power; and that as occasion may require, for the purposes of explanation, some of the planets may be conceived to revolve in centric orbits, 'and others in eccentric orbits, being nevertheless circles.

The line of the apsides is a right line extending through the centre of the sun, from the perihelion to the aphelion; hence the time of the apsides will be the diameter of the orbit, and may be thus denoted, even in a centric orbit; although such orbit cannot properly be said to have perihelion and aphelion.

In such system of planets, the squares of their periods would be as the cubes of their mean diameters from the sun. For as it is not necessary in order for a system of planets to fulfil this law, that the orbits should be alike eccentric, hence if some of them were centric and others eccentric, they would equally well fulfil the law; hence, if the lines of apsides of an eccentric orbit and of a centric orbit of the system were equal, the two planets would pass over equal areas in the times of their respective periods; the motion of one being uniform, and that of the other constantly varying inversely as the distance from the sun varies.

It will readily be perceived that as the force varies inversely as the distance, and the motion also, that those two elements preserve the same proportions to each other throughout the orbit, namely, the same in any other part of the orbit that they do in the mean points of force and motion.

And as the rate of convergency is inversely as the period, hence, the rate of convergency depends upon the velocity in one and the same orbit; so that if the velocity be double in one point of an orbit, in which the curvature is equal to what it is in another point of the orbit, the rate of convergency will also be double, \&c.

Hence then, those elements, namely, motion, force, and convergency, necessarily and consequently preserve the same ratio to each other throughout the orbit, that they do in the mean points; and hence the rate of force, or gravity, is a mean proportional between the rate of motion and the rate of convergency, throughout the entire orbit; nor are they under any necessity, as will be clearly perceived, of alternately getting the better of each other, in order to keep up the balance of power.

Thus the mean motion is inversely as the square of the mean distance; and the actual motion varies from the point of mean motion inversely as the distance varies; and hence, we deduce 
this general rule for finding the rate of motion at any given distance of the planet in its orbit from the sun, namely:

The product of the mean distance, by the rate of mean motion divided by the actual distance at any given point of the orbit, gives the rate of motion at such given point.

And upon no other principle can the motion vary inversely as the distance varies; nor the planet describe equal areas in equal times, nor the square of the period be the cube of the distance. And thus, gravity governs as perfectly throughout the orbit as though it were a centric orbit. There is no more necessity of calling to aid a centrifugal force, clothed with such powers as are found necessary to keep the balance of power; no more necessity of resorting to the popular method of accounting, by alleging that motion and force alternately get the better of each other, than if the orbit were centric; for the planet is as obedient to the force of gravity, and sings through the heavens with the same harmony and concord in the eccentric, as in the centric orbit.

Thus, in any eccentric orbit, the motion of the planet at any given point of the orbit varies inversely as the distance varies; hence, the motion at any given point of the orbit, will be the square of what the motion would be in a corresponding point of a like orbit to that of the given orbit, whose mean distance is the square root of the mean distance of the given orbit; because the mean motion of a planet is always inversely as the square root of the mean distance.

So if the mean distance of a planet be 4 , and its perihelion distance be 1 , the actual motion at any given point of the orbit, will be the square of what the actual motion of a planet revolving in a centric orbit would be, if revolving at just half the distance of such given point.

Thus if the mean distance be 4 , the motion at the mean distance will be $.5,-$ or the square of the motion of a planet revolving in a centric orbit, about the same central force, at the distance of 2 . And if the mean distance be 4 , and the perihelion distance be 1 , the motion at the perihelion will be 2 , or the square of the motion of a planet revolving in a centric orbit about the same central force at the distance .5 .

Let us now suppose a case or two : and firstly, suppose a planet revolving in an orbit whose mean distance is 4 , and whose perihelion distance is 1 . In such case we know the rate of mean motion is .5 ; the rate of mean force .25 ; and the rate of mean convergency.125; and the rates of motion, force and convergency, each, become quadrupled at perihelion, or when the distance has become 1-4th of the mean distance; that is, when the planet has arrived at perihelion, the rate of motion will be 2 , the rate of force 1 , and the rate of convergency .5; thus, retaining 
the same proportion to each other, and the same ability to perform their journey, or pursue the line of the orbit, as at any other point of the orbit.

Thus we perceive that the force from aphelion to perihelion, is constantly dividing its accumulating power between the motion and the convergency, according to their necessities; and from perihelion to aphelion, the force restrains and diminishes their rates in like proportions to that of their increase from aphelion to perihelion; and in such case the motion becomes accelerated and retarded, or rather increased and diminished, in the same proportion that the convergency becomes increased and diminished; or inversely as the distance is increased or diminished. Hence, the motion is not accelerated by the same law of progression, in respect to time and force, that a falling body near the earth is ; in which the progression of acceleration is constantly as the amount of the next succeeding odd number' is to unity.

Thus, when the foregoing planet arrives at perihelion, the force is the same as would be required for it to revolve in a centric orbit at that distance; but the rate of motion having become double what would be required for a planet to revolve in a centric orbit at that distance, the rate of convergency is consequently one half of what would be required; and hence, the planet pursues the curvature of the orbit dictated by the mean distance.

In the foregoing we perceive that the general rule applies, namely, the product of the mean distance, by the mean rate of motion, divided by the actual distance of any point of the orbit, gives the rate of motion at such point. So also this general rule is equally applicable to the rate of convergency, namely, the product of the mean distance by the mean rate of convergency, divided by the actual distance at any given point of the orbit, gives the rate of convergency at such given point; and this general rule also equally applies to the force.

This then is the simple and easy construction of the laws of force, motion, and convergency, in an eccentric orbit, based upon the hypothesis that force is inversely as the distance; but if we go upon the hypothesis, that gravity is inversely as the square of the distance, and append to it that extraordinary though prevalent version of Kepler's other law, namely, that the motion is inversely as the distance, (and without which appendage, even the idea or notion, that force is inversely as the square of the distance, could not have been sustained for a moment,) and take the foregoing planet whose mean distance is 4 , and whose perihelion distance is 1 , we shall find among others, the following results, namely:

The period must be 16 , and the mean rate of force .0625 , or 
the inverse of the period, and equal to the mean rate of convergency; and the mean rate of motion must be .25 , or inversely as the mean distance.

When the planet has arrived at the perihelion, the force will be 1 , or 16 times its mean; and the motion will be 1 , or four times its mean; and what should hinder the planet from then revolving in a centric orbit at that distance, may not readily be perceived; as the force and motion both conspire to that effect; there being no extra motion to cause a less rate of convergency than that of the force or motion; for it would be futile to say or assume that the rate of convergency was any other than unity, when there was no cause for it.

But it may be said that the actual period is 8 , the mean rate of motion .5 , and the mean rate of convergency 125 , and that those elements will always have the same proportions, whatever be the law of force; and hence, at perihelion, or distance 1, all the elements would have the same ratio to the rate of force, whatever be its laws; that in such case, the rate of force at perihelion would be a mean proportional between the rate of motion and the rate of convergency.

This mean proportional, it will be perceivéd, could only happen at the point of perihelion, and that too when at the distance 1. But in all other parts of the orbit, while the rates of motion and convergency preserved the same proportions to each other, and varied inversely as the distance, the force would be wandering, with little apparent regard to its change, over the motion and convergency.

But I have already fully shown that the only condition upon which the force can be inversely as the square of the distance, would be that the mean motion of a planet should be inversely as the mean distance, instead of being what it is, namely, inversely as the square of the distance; than which, a more plain or palpable fact could not well be desired.

I will now attempt to make some few simple applications of my determinations of the measures of the circle, to the phenomena of planetary motion, as corroborated by the great Keplerian law, and the eternal fitness of the economy of numbers for the development of the laws of nature, to the human understanding; - in which I will insist that the great Architect of the Universe had Unity of Purpose in view, when he created and established it by everlasting laws, which are as necessarily the laws of order, regularity, and the eternal fitness of things, as they are the laws of power and supremacy; otherwise we should discover but little of the attribute of wisdom; and hence, truth, when once discovered, may at least, be as beautiful as error. 
I will here remark, that the popular measures of the circle apply to the great Keplerian law, that the squares of the periods of a system of planets, are as the cubes of their mean distances from the sun, in the same manner as (and no better than) they apply to the measures of solids composed of plane surfaces. Hence, in applying the popular measures of the circle to the law by which a system of planets revolve in their orbits, as well as in the application of the circle to the measure of plain solids, it will always be found necessary in order to equate or finish out the work, to have on hand a sufficient number of minim quantities, as a kind of calculi to chink up with; and that too, in proportion as the extension of the work may serve to place it out of square.

I am not going to assume that the infinity of coincidences and reciprocities which continually flow from a comparison of my measures of the circle with measures of plain solids, or with the laws of planetary motion, as determined by Kepler's laws, is by any means to supply the place of absolute proof of the correctness of my determination; but such must depend upon the results and absolute conclusions drawn from an inductive course of geometric reasoning; in which one portion of the task will be to exhibit and show the errors of the popular methods, and thereby to endeavor to unloose that implicit faith in those errors which have become chained and riveted just in proportion as the inquirer has examined or attended to the subject; and for the accomplishment of which, it will be necessary in the consideration of the quadrature, to adopt certain appropriate (but very simple) signs, characters or symbols, which cannot here be used to advantage.

I will therefore simplify the present explanation as far as possible, - and will call 1-4th of the circumference of an orbit, the quadrant of the orbit; 1-8th of the circumference, the octant of the orbit, \&c. Radius of an orbit of course denotes one half of the diameter of the orbit.

I will notice that what is properly the prime orbit, is that whose radius is unity or 1 , having its diameter 2 , for the reason, that the squares of the periods of a system of planets, are as the cubes of their mean distances from the sun; and consequently when the distances of the planets of a system are numerically proportioned to any planet of the system, whose distance is assumed at unity; the square of the period of each planet of the system will be the cube of its own distance; consequently, when the distance of a planet is assumed at unity or 1 , its period is necessarily 1 . Nevertheless, what is properly the prime circle, is that whose diameter is unity, having its radius but half of unity. Such circle will be found to afford vastly more facili- 
ties for deducing the true quadrature, than any other numerical dimension.

But to proceed with a short application of my measures of the circle to planetary motion.

1. By the Keplerian law, the square of the period is the cube of the distance; or in other words, the period of a planet is the square root of the third power of the mean distance. And according to my measures of the circle, when the quadrant of an orbit is 4 , (or the circumference 16, ) the square of the quadrant of the orbit is the cube of radius. And this we know from Kepler's law, that when the square of the quadrant is the third power, or cube of radius, the quadrant of the orbit is consequently numerically equal to the period.

But by the popular measures of the circle, in order that the period may be equal to the quadrant of the orbit, the quadrant must be very little less than 4 , or the circumference of the orbit a very little less than 16 .

By Kepler's law the period of a planet is always the square root of the third power, or cube of radius; and by my determination of the circle, the quadrant of any orbit is numerically equal to the third root of the square of twice the period. Hence, when the quadrant of an orbit is 1, radius is the third root of .25 , and the period will be the second or square root of .25 , or equal to half of the quadrant, or to 1-8th of the circumference of the orbit; and we know that when the diameter of an orbit is 8 , the period is equal to diameter, or to twice radius; for then the third power of radius will be the square of the diameter as well as of the period. So by my determination, when the quadrant of an orbit is 2 , the period of the planet will be the square root of 2 , and radius of the orbit will be the cube root of 2 . And when the diameter of an orbit is 2, (namely, when the orbit is prime,) the period is necessarily equal to radius.

So when the diameter of the orbit is 4 , the period will be the square root, and radius the cube root of $8,-$ for it will be recollected that the period is always the square root of the same numerical quantity of which radius is the cube root.

Again, by my determination, four times the square of the period of a planet, is always the third power of the quadrant of the orbit; and radius of any orbit is always the farther of two mean proportionals from unity to the time of the period; for radius is always the third root of the square of the period.

So by my determination, when the period of a planet is .5 or half of unity, it is equal to half the quadrant of the orbit. When the period is 4 , the period is equal. to the quadrant of the orbit. When the period is 32 , it is equal to twice the quadrant of the orbit; and when the period is 256 , (or square of 16, ) the 
period is equal to four times the quadrant, or is equal to the circumference.

When the diameter of an orbit is 8 , the period is equal to the diameter; and when the quadrant of an orbit is 4 , the period is equal to the quadrant.

By the first allegation, the square of the diameter or the third power of the radius; and by the second proposition, the square of the quadrant, is equal to the third power of the radius.

To the first proposition, the world have assented, because it is plain to see, that the square of 8 is the third power of 4 . But in respect to the last proposition, by the popular measures of the circle, when the quadrant of an orbit is 4 , the period is 4.063750 , thus requiring a minim ratio of .063750 to equate the work with.

By my measures of the circle, the square of the period when the diameter of the orbit is the square root of 64 , is the third power of the period, when the quadrant of the orbit is the third root of 64 . Or thus, the square of the period, when radius of the orbit is 4 , is the third power of the period when the quadrant of the orbit is 4 .

But by the popular measures of the circle, the square of the period when radius of the orbit is 4 , would be the third power of the period when the quadrant of the orbit is a little less than 4, namely, 3.978988.

I will now just refer to my prime table, upon which I propose to explain many of the principles of the quadrature of the circle, for the purpose of showing its applicability to the laws of planetary motion also. Hence, if we take any term of the table for the mean distance of a planet, we readily obtain in other terms of the table, the period, the rate of mean motion, the mean rate of force, and the mean rate of convergency of the planet, - as also the whole amount of motion, of force, and of convergency during the period of the planet; the amount of force during an entire period being always a mean proportional between the amount of convergency, and the amount of motion during the period.

Thus, if the mean distance of a planet be the third root of 4 , for instance, the time of the period will be the third or cube root of 8 , the rate of mean motion will be the third root of .5 , the rate of mean force will be the third root of $: 25$, and the rate of mean convergency will be the third root of .125. The whole amount of "convergency during the period will be 1 , the whole amount of force during the period will be the third root of 2 , and the whole amount of motion during the period will be the third root of 4 . 


\section{H A P T E R IV.}

\section{On the Hypothesis suggested by Kepler in respect to Elliptical}

Orbits.

Perhaps one reason that may be offered why it is not as imperious upon philosophy and astronomy that the elliptical hypothesis of Kepler should be adopted, as that his other two laws should, is, that any general law of gravity or motion, applies equally well, at least to an eccentric circular orbit, as to the ellipse.

Thus Newton's famous demonstration of Kepler's hypothesis, that the motion of a planet revolving in an eccentric orbit varies inversely as the distance varies, and hence that the radius vector will pass over or describe equal areas in equal times, by an application of the problem, "That triangles on the same base, or on equal bases, having the same height are equal to each other," is equally applicable to a circular orbit, whether centric or eccentric, as to the ellipse; nay further, it applies equally well to the parabola, or hyperbola.

Hence there appears to be no necessity that a planet should revolve in an ellipse with the sun in one of the foci, in consequence of such demonstration; as it can equally well revolve in conformity with such demonstration, in a circle, having the sun stationary in any given point within the circumference of the orbit.

Nevertheless, it is not so much my intention to establish the particular forms of orbits, as to promulgate the true laws of gravity and motion; to endeavor to reconcile their complicated simplicity, and to remove that blur which has so long perplexed the student and inquirer when endeavoring to get an insight into the laws of force and motion through the medium of those round and round explanations given by Maclaurin, Ferguson, and others; by which we are given to understand, that the velocity sometimes gets the better of the force, and sometimes the force gets the better of the velocity, and that it has been found necessary, in order to regulate or equate the defect in the 
eternal laws, to institute, as a kind of balance wheel, what they term the centrifugal force, which, by the aid of such forces as they choose to bestow upon it, enables them to make the requisite equations.'

Mr. Norton, in his Astronomy, gives the following version of what are called Kepler's three laws, viz:

1. The areas described by the radius vector of a planet are proportional to the times.

2. The orbit of a planet is an ellipse, of which the sun occupies one of the foci.

3. The squares of the times of revolution of the planets are proportional to the cubes of their mean distances from the sun, or of the semi-major axes of their orbits.

And Mr. Norton says that "The first two Kepler assumed as hypotheses, (on partial examination,) after he had discovered that the radius vector and angular motion of a planet were equable, and afterwards verified them, or partially verified them, - and they have been completely verified by other astronomers," - and promises to show in the sequel, that they are verified by the results deducible from them; and remarks, "that these two laws being established, the third is obtained by simply comparing the known major axes and times of revolution," and then alleges, that " the apparent motion of the sun in space must be subject to Kepler's laws."

That the apparent motion of the sun in space is subject to Kepler's first and third laws, there can be ho doubt. But that it is subject to the second, must depend upon the proof of the elliptical hypothesis; and that must, probably, be deduced from observation alone, if proved at all ; as no physical cause appears to come forth in support of it.

Mr. Norton says, "The orbits of the sun, moon and planets being regarded as ellipses, the perigee and apogee, or the perihelion and aphelion, are the extremities of the major axis of the orbit."

This is true, if we regard the orbits as ellipses; for the ellipse is what presents a major axis, the extremities of which must be the apogee and perigee. But it is the eccentricity of the orbit which gives the apogee and perigee, and this whether the orbit be an ellipse or a circle.

Mr. Norton again says, "The law of angular motion of a planet about the sun, may be deduced from Kepler's first law."

This with proper qualifications is doubtless true; but it has nothing whatever to do with the elliptical hypothesis, as it depends wholly upon the eccentricity of the orbit, and not upon the ellipticity; for when the orbit is eccentric, what astronomers 
call the angular motion, must of necessity exist. And if the actual motion varies inversely as the distance varies, the radius vector must of necessity describe equal areas in equal times, whatever be the form of the orbit; and this in accordance with Newton's said demonstration, from which so much Astronomical calculation has been drawn; as that bodies may form their perihelions in parabolic or hyperbolic curves; and may revolve about the sun in all kinds of ellipses however eccentric.

Nor do I think that Mr. Norton's explanations, given in Sections 184 and 185 of his Astronomy, have improved at all upon Newton's demonstration, or that they in anywise prove that an eccentric orbit must of necessity be an ellipse. Nor does he even pretend to deduce the angular motion from any other cause than that of the eccentricity of the orbit; for he only assumes or considers the orbit used by way of explanation, to be an ellipse.

Thus far it would seem as though nothing had been shown, proved, or demonstrated, in favor of the ellipse, that does not equally apply to the eccentric circular orbit; and that the elliptical hypothesis must still rest for its support upon evidence to be wholly drawn from observation, (if supported at all,) in lieu of being drawn from any physical cause that can be assigned.

But so general is the belief in the elliptical hypothesis, that there remains perhaps as little apprehension that the orbits are not ellipses at the present day, as that they were so previous to the days of Kepler. And hence we find the constant attempt in all astronomical works, by almost every mode of expression that can be devised, to induce a belief in the elliptical bypothesis ; and often, too, in a way and manner that would seem not to be required for the purposes of explanation of the subject directly under consideration, - as though it were an imperious point of faith, however reluctant the mind should be to adopt it.

Thus Mr. Norton says, "The eccentricity of an elliptic orbit, is the distance between the centre of the orbit and either focus;" and if a circular orbit be eccentric, the eccentricity is the distance between the centre of the orbit and the sun; the same as in the ellipse.

Again he says, "The most accurate method of determining the longitude and epoch of the perigee, rests upon the principle that the apogee and perigee are the only two points of the orbit whose longitudes differ by $180^{\circ}$, in passing from one to the other of which the sun employs just half of the year. This principle may be inferred from Kepler's law of areas; for it is a well known property of the ellipse, that the major axis is the only line drawn through the focus, that divides the ellipse into 
equal parts, and by the law in question equal areas correspond to equal times."

Now the manner in which this sentence is constructed, has somewhat the appearance of a design to sustain a favorite theory which is lacking in the requisite proof, and hence must be sustained by presumptive evidence however futile.

Now not a single important fact alleged in the sentence arises from the properties of the ellipse; for the truth is, that in either an ellipse or a circular orbit having eccentricity, the apogee and perigee are the only points of the orbit whose longitudes differ by $180^{\circ}$ in passing from one to the other, of which the sun employs just half the year; and that the line of the apsides is the only line that can pass through the sun and divide the orbit into equal parts at the same time, is as true of the eccentric circle as of the ellipse. And the proof arising from the application of Euclid's problem in support of Kepler's first law, - viz, "That if a body be made to revolve around a fixed point with a motion varying inversely as the distance, a line drawn from such fixed point to such revolving body, will describe, or pass over, areas proportioned to the times," - is as true in respect to the circle as to the ellipse.

Having consulted Mr. Norton thus far, I am unable to discover that he has furnished the slightest evidence in favor of the elliptical hypothesis; notwithstanding we find from his preface, that, in his compilation, he has consulted the most eminent authors upon astronomy and mathematics; and hence, thus far physical astronomy does not seem to furnish equal evidence in support of the elliptical hypothesis that it does in favor of Kepler's other laws. And hence the evidence in support of said hypothesis seems rather to be the result of Kepler's observations, to which an easy or ready assent has been given; and especially since the celebrated demonstration in respect to equal areas in equal times; which seemed to operate as a charm, even upon matters with which it had no concern.

But it is possible that the consequences resulting from the adoption by Kepler, of the elliptical hypothesis, in connection with Newton's demonstration of Kepler's first law, and his especial application of it to Kepler's elliptical hypothesis, are not such at the present day, in physical astronomy, as a better dispensation would have produced; for the problem applying equally well to an ellipse of extreme eccentricity as to any other, neither Newton, Halley, nor the world, have set any bounds to the ellipticity of their orbits. For although both Newton and Halley, previous to the supposed important demonstration, supposed the comets to perform their perihelions in parabolic curves, 
they changed their views after such demonstration, even in respect to cometary motion.

They hence allow 575 years for the period of the comet which appeared in 1680, and give it an elliptical orbit so eccentric that its curvature at its perihelion must be very rapid indeed, it being 166 times nearer the sun than the earth is. And notwithstanding the astonishing velocity with which it is said to move in its perihelion, yet if the orbit were an ellipse with the sun in one of the foci, the ellipticity would be so great or extreme, that if another comet were to revolve in a circle with its line of apsides of equal length and of equal eccentricity, (and Mr. Norton says that the line of the apsides is what determines the length of the period,) the motion of the comet revolving in the ellipse would be but about $2-3 \mathrm{ds}$ as great as that of the comet revolving in the circle, in any corresponding part of the orbit, - as will be self-evident to any one who will examine the subject but for a moment; while the amount of the force of gravity applied to the comet revolving in the circle in the time of its period, would, upon any hypothesis of the law of gravity, be vastly less than the amount applied to the comet revolving in the ellipse in the time of its period, or in the same time.

But this part of the subject belongs more particularly to the laws of gravity and motion.

But notwithstanding the elliptical hypothesis is such a favorite in the science of astronomy, it is somewhat surprising that it should be so, seeing its application is so repugnant to, and irreconcilable with, the laws of gravity and motion; for though it is alleged that the orbits of sorne of the comets are known from observation to be very eccentric ellipses, yet there are but three, namely, Eucke's, Biela's, and Halley's, whose periods and orbits have been determined.

But it is alleged by Mr. Vince and others, that astronomers do not calculate the elements of a visible comet upon the principle that the orbit is an ellipse, but upon the principle that it is a parabola, and afterwards deduce the elliptical form from those calculations.

The elliptical hypothesis, then, seems to receive but little, if any aid, by its application to cometary motion, it being sustained neither by any known physical laws, nor by observation upon which dependence can be placed.

Let us, then, recur to what Mr. Vince says in respect to Kepler's investigations of the subject, and which induced him to adopt and promulgate the hypothesis.

Ptolemy supposed that the orbits of the planets were circles, and that the earth was not in the centre of those orbits, but situ- 
ated at some distance from the centre of those orbits, around which the planets revolved with an equable motion; and thus he endeavored to account for the apparent inequality of a planet's motion, requiring therefore an equation of its orbit.

Tycho adopted this theory in part, retaining the theory of the equable motion of a planet around a centre, but placed that centre at a different distance from the earth from that at which Ptolemy placed it, by which his observations and computations would agree within a few minutes; but as the eccentricity, calculated from the equation, would not agree with his supposition, he concluded that the sun was not always at the same distance from the central point, which made Kepler suspect that the centre was not the point about which the motion was equal.

Hence Kepler instituted a series of calculations upon the orbit of Mars, based mostly upon Tycho's observations, for the purpose of ascertaining its eccentricity upon the assumption that it was a circle; and calling the distance of the earth 100000 , he found the aphelion distance of Mars to be 166780, and the perihelion distance 138500 , and hence its mean distance 152640, and its eccentricity 14140.

He next determined three other distances of 'Mars, and found them to be 147750,163100 , and 166255; and on calculating their distances upon the supposition that the orbit was a circle, he found that they would corne out 148539,163883 , and 166605 , and that the errors, upon the hypothesis that the orbit was a circle, would be 789,783 , and 350 . The distances determined being, to those calculated upon the ground that the orbit was a circle, as 1 to 1.0053 , as 1 to 1.0048 , and as 1 to 1.0021 .

But Mr. Vince says that "Kepler had too good an opinion of Tycho's oisservations to suppose that this difference might arise from their inaccuracy; and as the distance between the aphelion and perihelion was too great, upon the supposition that the orbit was a circle, he knew that the form of the orbit must be an oval or ellipse, with the sun in one of the foci; and upon calculating the said observed or determined distances, he found they agreed together. He did the same in regard to other parts of the orbit, and found they all agreed; and thus he pronounced the orbit of Mars to be an ellipse, having the sun in one of the foci.

Having determined this for the orbit of Mars, he conjectured the same to be true of all the other planets, and upon trial he found it to be so. Hence he concluded that the six primary planets revolve about the sun in ellipses, having the sun in one of the foci."

Mr. Vince says, "that Kepler, in calculating the eccentricity 
of the orbit of Mars, upon the hypothesis that it was a circle, and on Tycho's principle that it had an equable motion around a point, (supposing the sun and such point were bisected by the centre of the circle,) and having calculated twelve oppositions of Mars observed by Tycho, none of which differed more than $1^{\prime} 47^{\prime \prime}$, he found that the hypothesis did not agree with the latitude observed in opposition, nor with the longitude out of opposition, which differed sometimes 8 ' from observation; from which want of agreement, and from the place of the sun, and the point around which the motion was supposed to be equable, not being bisected by the centre when thus computed, he was persuaded that the orbit of Mars was not a circle."

But it is said that the circle, which Kepler says so well represented the twelve oppositions observed by Tycho, and on which he based his calculations, had an eccentricity equal to 18544 , between the sun and point around which the motion was supposed to be equal, but he found this quantity to be divided by the centre of the orbit as follows: between the sun and centre of the orbit, 11332; and between the centre of the orbit and supposed point of equable motion, 7232 .

Now whether there was error in Tycho's observations, whether there was error or discrepancy in Kepler's calculations, or whether they may have been wrongly quoted, I know not. But in one case upon Tycho's observations, Kepler finds the eccentricity of the orbit of Mars to be 14140, (calling the distance from our earth to the sun, 100000 ;) and in another case upon Tycho's observations, he finds the distance between the sun and centre of Mars' orbit, to be 11332, - a difference of near 3000 in the two calculations, the double of which subtracted from the line of the apsides, as by him first determined, would serve doubtless to fully compensate his oval or ellipse into a circle, according to his determination of the three distances in other parts of the orbit.

But without more light being thrown upon this intricate subject,- - depending, as it must of necessity, upon observation alone for its support,-it is impossible to tell whether Kepler, in his investigations from which emanated his elliptical hypothesis, detected and rectified error to a greater extent than he established it.

And notwithstanding we have all looked upon Kepler as the father of physical astronomy, and that he has done more in laying its foundation than all who have attempted its superstructure, and that it is no idolatry to admire such wisdom in an earthly shape,- nevertheless, as the elliptical hypothesis seems to be wholly abstract from any known physical law, or necessity connected with unity of purpose or harmony of design, depend- 
ing for its support upon observation alone, let it again be oorrectly examined, and if found to be true, it is doubtless of more extended consequence than we have heretofore conceived it to be.

But Kepler himself was somewhat perplexed with his elliptical hypothesis; for, although his first law, - namely, that the motion varies inversely as the distance varies, - furnished the means for equating an orbit, yet, upon the elliptical hypothesis, the finding of the true anomaly from the mean, or the mean anomaly from the true, was no easy task; for Mr. Vince says, "that although Kepler suggested a problem, which still goes by the name of Kepler's problem, for finding the mean anomaly from the true, and vice versa, of a planet revolving in an ellipse, still the problem has remained unsolved to this day, otherwise than by approximation, notwithstanding it has exercised the talents of eminent mathematicians."

Such, then, is the evidence upon which the elliptical hypothesis rests; and if it be of consequence to know whether it be true or false, it is also of consequence that observations, depending, as they necessarily must, upon much intricacy and exactness, should be so critically, repeatedly and carefully made, as shall leave no room to doubt.

Not being able to call to aid any evidence in support of the elliptical hypothesis, I will endeavor to make some few comparisons between the ellipse and the eccentric circle, as orbits in which planets may be conceived to revolve, for the purpose of seeing if any light can be shed upon the subject by such a course; and here I will remark that in such examples or comparisons, one and the same central power is conceived to be used for all the examples.

Conceive, then, an eccentric circular orbit, whose distance is 8 ; of course, the line of its apsides is 8 ; and let its perihelion distance be .25 , and its aphelion distance be 7.75 , its motion at perihelion would of course be 4 , and its motion at aphelion, $.2666,-$ or $1-15$ th part as great as at the perihelion. Now suppose an ellipse to be inscribed in the circular orbit, their respective perihelions and aphelions touching each other, with the sun in one of the foci of the ellipse. That is, according to the idea of an elliptical orbit, it shall be as elliptical as it is eccentric. Thus at the perihelion and aphelion, the rates of gravity will be the same in both orbits, but will be greater in the ellipse than in the circle in all parts of the orbits excepting those two points.

For by Kepler's third law, the periods of the planets must be the same, because the line of their apsides are equal; at least, according as Mr. Norton renders the law. 
Each planet in respect to itself, would describe equal areas in equal times; nevertheless, the planet revolving in the circle would not only pass over more area than that revolving in the ellipse, in the same time, but more in proportion to the velocity or space passed over.

As the rate of gravity of the planet revolving in the ellipse would in all parts of its orbit, or at all times, be greater than that of the planet revolving in the circle; hence, its amount of gravity in the time of its period would be greater than that in the circle.

Hence, we have the strange anomaly, of two planets revolving in equally eccentric orbits around the same central force, with their mean distances equal; their gravities equal at perihelion and aphelion; and yet one of them constantly passing over but about two thirds of the space of the other, and a far less proportion of area, in the same time, and yet constantly urged towards the central power by a far greater force of gravity.

Now if planets were made to revolve in those two orbits, they would have certain principles in common with each other; in each the product of the mean distance by the mean motion, divided by the actual distance at any point of the orbit, would give the rate of motion at such point; so each, in respect to itself, would describe equal areas in equal times, if made to revolve by a motion varying inversely as the distance; for the principles are only reciprocal conditions of each other.

I will now suppose two orbits situated similar to the foregoing orbits, the lines of their respective apsides being 2 , (hence the mean distance of each, according to Newton, is 1,) and let their eccentricities be such that the circumference of the ellipse is just 2-3rds that of the circle; for if planets can revolve in all kinds of ellipses, however eccentric, they can revolve in such as the foregoing.

In such case the rate of motion in the ellipse in corresponding points of the orbit, should always be just 2.3rds as great as that in the circle, and hence must be so in their point of mean motion. But if it be not so, we have no criterion whereby to proportion the rates of motion between the two, any more than we have whereby to obtain the true anomaly from the mean, or the mean from the true, of a planet revolving in an ellipse; in fact, it would be all anomaly.

But what seems somewhat perplexing in respect to the elliptical hypothesis, is, that it seems always inclined to break the law, and will not be subject to it; but having fortified its position behind the supposed correctness of 'Tycho's observations, and Kepler's calculations made from the same, it has thus ac- 
quired a kind of invulnerableness to an attack, by any legitimate kind of warfare.

Thus by a universal law of force and motion based upon Kepler's great law, that the squares of the periods are as the cubes of the mean distances, we find that the mean distance of a planet divided by the mean motion gives the period; or the mean distance divided by the period gives the mean motion. And although we find this law to prevail in a circular orbit, whether centric or eccentric, it is always broken in the ellipse, whether the eccentricity be much or little.

Thus, in the two last-named orbits, if the periods and mean distances must be equal, they will each of necessity be 1 ; and if the mean distance of each be divided by the period, the result will be the same in each, viz., unity or 1 ; but it will not give the mean motion in the ellipse, for that is but 2-3rds as great as in the circle.

So if the mean distance of each be divided by the mean motion, the period in the circle will come out but $2-3 \mathrm{rds}$ as great as that in the ellipse. So that in either case, we find error, and a want of conformity to the laws of force and motion. But suppose in the two last-named orbits, that both planets move from the perihelion at the same time, and that at perihelion, the motion of the planet in the ellipse is just two thirds of that in the circle.

Now while we are making planets revolve as we please, we may imagine that the ratios of the motion of the two, will so continue to aphelion, and throughout their orbits; and such would be the case if both planets were constantly attracted by the same intensity of force; for in such case, they would preserve at the same height the same ratios or proportions of motion, whatever might be their respective routes in arriving at such height; at least, according to Sir Isaac Newton; for Mr. Maclaurin says, (Book 4th, Sect. 21,) "Our author (Sir Isaac Newton) has shown that if one body move in a curve, and another ascend or descend in a right line, acted on by the same gravity, and their velocities be equal in any equal altitudes, they will be equal in all other equal altitudes." Hence, if it were possible to make the application of what " our author has shown," (which by the way it is not; for I believe that no one can well suppose that if the motions of the two planets were equal in perihelion, that they could take their respective routes in their orbits, having their velocities equal throughout their respective orbits, and at the same time perform their respective periods in the same time,) it would serve to establish my premises; for the amount of gravity applied to the planet in the ellipse in the time 
of its period, is vastly greater than what is applied to the other; and hence I will leave it to others, either to reconcile the incongruities, or to complete the demonstration by way of reductio ad absurdam.

The admiration which the wisdom of Kepler so justly merits from the world, ought not to be bestowed indiscriminately without regard to truth and error; and we should as readily come up to the help, against error which he may have promulgated; as if promulgated by any other person.

I have already stated that his hypothesis in respect to elliptical orbits is not founded upon those immutable laws, which may be mathematically or numerically determined, as in respect to his other two laws, but rests upon the supposition that Tycho's observations, as well as his calculations made thereon, were wholly correct; and that there could have been no error, either direct or collateral in either. Nevertheless, while his other two laws, which are immutable and eternal in the heavens, have as yet been more or less misapprehended and misapplied, his hypothesis in respect to an elliptical form of the orbits, has not only taken deep root, but has grown to the overshadowing of every other principle in respect to the planetary system.

Nor does it appear that Kepler, or others, who have so industriously inculcated the elliptic hypothesis, have been aware that it could not be true, in case the motion of a planet in its orbit varies inversely as the distance from the sun varies.

The ellipse or elongated circle, may vary from that of the circle, to one of extreme ellipticity, in which the two sides will differ but little from straight lines; and the distance from either focus of the ellipse to the nearest or adjacent end or vertex, is always less than half of the shortest axis or diameter of such ellipse; and it is gravely taught as a philosophic truth, that a planet may revolve in an ellipse, however elliptical; and also that the motion of such planet, will vary inversely as the square of the distance from the sun varies.

Now it would seem that the simple mention of these two positions in connection, would be sufficient to show the important error contained therein, without any attempt whatever at further explanation; and that Kepler could not have committed the error, in case the law of falling bodies had then been disclosed by Galileo, his contemporary. But that Newton, who seems not to have been culpable for any error or imposition which he might entail upon the learned world, should have committed the error, with the law of falling bodies before him, (not to say with the full knowledge of the law of falling bodies,) is to me not at all surprising. 
For notwithstanding his attempts at a disclosure of the law of gravity from a consideration of the law of falling bodies, (the very process which should have detected the fallacy of the elliptic orbit,) the bungling and palpable errors therein committed might well induce the belief that no error was too palpable or gross for him either to cornmit or overlook.

The ellipse, or elongated circle, may vary from the circle by any assignable quantity; for as Newton would say, Neque novit natura limitum.

If a planet revolve in a centric orbit, (viz., with the sun in the centre,) the motion will be uniform; the whole amount of centripetal force during the time of the period, being expended in producing the convergency of the planet; and consequently, no portion of the force is expended in accelerating or retarding the motion of the planet.

If such orbit shall become eccentric, (still retaining its circular form,) inasmuch as the planet in the course of its revolution approaches and recedes from the sun, then upon the principle of falling bodies, its motion must alternately be accelerated and retarded, and consequently a portion of the constant force applied is expended in such acceleration and retardation, and the remainder in the convergency.

Now the convergency of a planet is no part of the motion, and may simply be defined to be the direction of the motion; and so we find the definition in treatises upon the involution and evolution of curves, where it is said that "curvature is a change of direction only," although it is frankly acknowledged in those treatises, that curvature has been illy defined, and the subject very much perplexed in its consideration. Nor is it passing strange that the subject should have been perplexed, considering the manner in which it has been treated.

I have heretofore attempted to show that convergency is not only a proper, but an important element in the consideration of planetary motion; that it is a tendency to the same point again, from which the planet has revolved; that in the time of the period, the convergency is always $360^{\circ}$, and consequently the amount of convergency in the time of the period is always unity, and hence might be assumed as a standard element in any orbit, whatever may be the distance; that it is directly connected with planetary motion and the law of gravity; and that the rates of motions, of gravity, and of convergency, are so proportioned to each other, and to the period and distance, as to perform the grand results required by the Keplerian law. That the mean rate of convergency is always inversely as the time of the period, - consequently, inversely as the cube of the square 
root of the distance, - it is hence, the cube of the mean rate of the motion, and the cube of the square root of the mean rate of the gravity or force, \&c. And hence, the mean rate of gravity or force, is always a mean proportional between the mean rate of motion, and the mean rate of convergency; and gravity throughout the period is equally attentive to convergency as it is to motion; and whether. the orbit be centric or eccentric, the element of gravity or force, at all times and in all parts of the orbit, preserves the same position in respect to the elements of motion and convergency, viz., a mean proportional between them; for upon no other condition can the motion of the planet vary inversely as the distance varies from the sun; nor should we be much alarmed at the immutable precision by which the force is thus divided or proportioned.

Suppose, then, the force not to be thus proportioned between the motion and convergency; that is, suppose the orbit to be an ellipse, in which case it is manifest that the force cannot be thus proportioned to the motion and convergency. And to lay our premises broad, we will suppose such ellipse to possess very great ellipticity; in which case the planet, in descending from its aphelion to its perihelion, shall describe a line of but little curvature, or varying but little from a rectilinear fall; in which case, the motion of the planet at aphelion will be as near to 0 as the ellipticity of the orbit is to extreme ellipticity.

Now the rates of commencement of motion, or of continued motion of two bodies falling from a state of rest at the same time from different distances, towards the same centre of gravity, are inversely as the squares of the distances at which they commence their respective fall, for the very reason that the force of gravity is inversely as the distance; so also, the motion of each body from the commencement of its fall, will vary inversely as the square of the distance varies; and such would be the condition of a body revolving in the extreme ellipse, (if, however, such impossibility could be performed; but I will not, like Maclaurin, be wiser in respect to laws that have no existence in nature, than those which have.)

The consequence must then necessarily be, that as we vary from the circular orbit, whether centric or eccentric, in which the motion varies inversely as the distance varies, and approximate from the ellipse of least ellipticity to that of the greatest, we must necessarily approximate from the inverse variation of the motion to the distance, to the inverse variation of the motion to the square of the distance.

I have, therefore, in my investigations, become rather satisfied that no other explication conld wel: be given of the opera- 
tion of force and motion as applicable to a planet revolving in an ellipse, than that given by Maclaurin and others, viz., that the force and motion alternately get the better of each other. And whether it shall continue to be said, as heretofore, that, "As it has been proved that a planet can revolve in an ellipse, having the sun in one of the foci, it consequently follows as a general law, that bodies may revolve in all kinds of ellipses, however eccentric," or, whether it shall hereafter be said that, as it has been shown that bodies cannot by the laws of force and motion, revolve in all kinds of ellipses, however eccentric, it consequently follows that a body cannot, by the laws of force and motion, revolve in any kind of ellipse, - are questions which are submitted for consideration. 


\section{$-$ \\ C H A P T E R V. \\ Outline of a Theory of the Solar System.}

I have already intimated that I might offer an outline of my theoretic notions in respect to the great physical law of the universe, under its various modifications, in its constant operations in the adjustment and control of the Solar System; referring for evidence in support of such theory, so far as I am able to avail myself of them, to such facts, phenomena and analogies in nature, already before the world, as will, in my opinion, best serve to sustain or support such theory or hypothesis.

In assigning causes to the phenomena of nature, perhaps we cannot well adopt a better criterion by which to be governed, than that contained in the first two rules laid down by Sir Isaac Newton, namely: "No more causes are to be admitted than are sufficient to explain the phenomena;" and 2d, "Of effects of the same kind, the same causes are to be assigned, as far as it can be done." And it might be well for the advancement of science and philosophy, were these rules more strictly adhered to. But we are all quite too apt to deviate from those rules which we may have prescribed for our future conduct.

But in attempting to theorize where the immutability of numbers does not come directly to our aid, there are many things as yet too high for our understanding, - too vast for our comprehension,- the causes too remote to be assigned, and the operations too abstruse and evasive in their detection, to be well defined or explained, without more facts and data than the accumulated knowledge of the world is yet in possession of, to enable us to form a theory (even of the Solar System,) that is not in many respects liable to constant demolition, arising from the discovery of new facts and analogies; all of which, doubtless, tends to show the simplicity, harmony, reciprocity and analogy which pervade all the laws of nature, and their operations. 
I will not here make the sacrilegious attempt to theorize upon the primitive formation of the universe, as many have done, for the purpose of accounting for all things; or, at least, of being understood by others to have done so,- as how adventitious nature, with her gravity on hand, accumulated her atomic matter into its present aggregate masses, from which gravity is constantly emanating in search of prey, and that nothing but the speed of the smaller bodies prevents their being dragged to the larger, - or the no less vague, unwarranted and uncalled for assumption that all the revolving bodies of the solar system were originally projected in tangents to their respective orbits, with such forces as would produce the present momenta of the revolving body in that point of the orbit where it was first taken charge of by gravitation, whatever point in the orbit that might have been - so that all the people might know by what means the planets and other revolving bodies first got under way. But as none of these projections have happened in our day, our faith in the hypothesis (if we have any) must rest upon a supposition or apprehension which is of no use whatever to science, as it is not a phenomenon of nature or of nature's laws; we see it not, we know it not, and at best it is but an attempt to show a necessity, consonant with human contrivance, which Deity was under, of making use of certain means for the accomplishment of his purposes in creating and upholding the universe.

Not so with the laws of nature, which are continually operating, and the phenomena which they produce, and which it is our highest privilege to investigate, thereby exalting the human mind by a more comprehensive view or understanding of cause and effect, which, more than anything, distinguishes man from the brutes.

Nor will I undertake to fancy that this earth, at some former period, was detached from the sun in one molten mass, the outside of which as yet is but just cooled to a crust, and that we are yet in danger of having it broken up in some terrible eruption, and we plunged into the eternal fires, \&c.

But I would rather confine my speculations to the solar system, as it now exists, and as it now appears to be governed by those immutable laws founded in the fitness of things, and from which all the phenomena of nature are doubtless results, effects and consequences.

Of gravitation, or the attraction of gravitation, we at present know but little, otherwise than through its effects and operations upon palpable matter, or matter in aggregate form. Nor is our philosophy sufficiently astute to reason intelligibly upon the operations of gravity, or attraction, upon two inconceivably small 
corpuscles or physical atoms, and thence to transfer the analogies of those operations to that of tangible masses of matter, as has been attempted and pretended by high philosophers.

Nevertheless, we have sufficient facts, data and phenomena, resulting from the laws of gravitation or attraction, from which to deduce many important results, if analogically considered, amongst which is that of the ratio in respect to distance, by which the attraction of gravitation operates upon matter. But in what way or manner the influence of gravitation is exerted on matter, has perhaps never been well defined or determined. Nevertheless, it is not absolutely necessary that we should conceive it to operate through absolute space, without a material medium in and through which to operate; and which medium, however attenuated, has a density varying in some given ratio to the distance from bodies of aggregate matter, namely, in the same ratio as that of the force of gravitation.

Perhaps there are but few astronomical facts better ascertained from observation and calculation, than that the attractive powers exerted by the several primary planets of the solar system, are as their bulks or magnitudes directly, and their inverse distances from the sun conjointly. And if such be the case, it furnishes strong presumption, at least, that their attractive powers are not innate, and that attraction or gravitation is not an essential property of matter; but that the planets of the system obtain their gravitating and attracting powers from the circumstance of their being within the attracting power of the sun, in the same manner as soft iron obeys the magnetic influences when brought near a permanent magnet or an electro-magnetic battery.

I know that Sir Isaac Newton, having adopted the theory of the equal and innate gravity of all matter, and observing that the attracting power of Jupiter and Saturn upon their respective satellites was not as great in proportion to their apparent magnitudes as that of the earth upon the moon, adopted the concomitant hypothesis, that those primary planets are less dense than the earth is. And such has since been the progress in ascertaining the different densities of the primary planets, that Euler calculated their densities to be inversely, as their distances from the sun; and the astronomer and mathematician, LaGrange, long since adopted the same ratio, as a standard from which to determine their disturbing forces upon other matter; and after much observation on the subject, remarked that he saw no cause for altering that ratio, believing it to be true.

To be sure, the present Herschel supposed the planet Herschel to have a greater density than would be given it by such 
ratio, but modern observations have determined that he overrated the density of Herschel.

But I know of no analogies in nature to sustain the hypothesis of the different densities of the planets, and shall therefore endeavor to seek for the cause of the phenomena upon which such hypothesis is based, in the analogies arising from observed phenomena, as well as such direct proof as may have been adduced by those who have been most forward in establishing and sustaining the hypothesis of the different densities of the planets. But surely there can be no analogy between the hypothesis that the force of gravity varies inversely as the square of the distance, and that which assumes the densities of the planets to be inversely as their distances from the sun. But that there are analogies between the hypothesis that the force of gravity varies inversely as the distance, and that the planets vary in their respective gravities in proportion to their respective magnitudes, in the inverse ratio to their respective distances from the sun, I will endeavor to show.

In respect to the Newtonian law of gravity, namely, that gravity varies inversely as the square of the distance, we find in Vince's History of Astronomy, Vol. 2, p. 279, the following, namely:

"That in 1684, Dr. Halley turned his thoughts to the relation between the periodic times and distances of the planets, and concluded from it that the centripetal force must vary inversely as the squares of the distances; but not being able to prove it, he applied to Mr. Hook and Sir Christopher Wren; they, however, not being able to give him satisfaction, he went to Cambridge, to Mr. (afterwards Sir Isaac) Newton, who soon gave him a proof of his position."

And this a proof, as also that of Newton's theory of universal gravity, seems not only to have satisfied the mind of Dr. Halley, who was searching for truth, and longing to be satisfied, but also that of the world in general; and it appears that no farther inquiry was made upon the subject. Thus we find in the American Encyclopædia, under the head of Gravity, that the author asserts, that gravity appears to be an essential property of matter; that it is proportional to the masses of bodies; that it varies inversely as the square of the distance in proceeding from the surface of the body outwards, or from its centre; that it varies directly as the distance, in descending from the surface to the centre in uniform spherical bodies; and that it is transmitted instantaneously from one body to another, \&c. Some of which allegations are certainly worthy of consideration and remark in due time. 
But a vast amount of what is generally conceived by the world to be evidence of the law of gravity, as declared or promulgated by Sir Isaac Newton, may be found in Vince's Physical Astronomy, Vol. 2, Chap. 31, under the head of "The general principles of centripetal forces," in which frequent reference is made to the Principia, as authority; and in which, to me, the reasoning appears to be vastly more deep or dark, than profound; as by attempting to geometrize upon a geometric point, or upon the law of gravity which two geometric points or physical atoms will regard in consequence of their innate gravity upon each other, aided by the use of expressions like the following: the limiting state, the limiting ratio, a ratio of equality, ultimately proportional, \&c., - which being applied to the circular orbit, the transition is then made to the ellipse, the parabola, and the hyperbola. (And, perhaps, no one will doubt but that the same law of gravity which operates in the circle, will operate also in the ellipse, the parabola, and the hyperbola, so far, at least, as distance is concerned.) And in the result, we find Mr. Vince coming to the following conclusion, viz.: "Hence, we conclude that each planet is attracted to the sun by a force which varies inversely as the square of the distance of its centre, and that the constituent particles also attract each other by a force varying according to the same law," and that the measure of force will be obtained by dividing the square of the motion by the distance; which, if true, would consequently make the force inversely as the square of the distance; which explanation has too well satisfied the world.

I will now proceed to a consideration of the attractive power of the planets as proportioned to their magnitudes and distances from the sun.

Mr. Vince says, "That to understand the principle upon which this determination rests, we may observe that the effect of attraction at equal distances will be in proportion to the quantity of matter in the attracting body; and at different distances, as the quantity of matter and the inverse square of the distance conjointly. The quantity of matter is also in proportion to the magnitude of the body and its density conjointly. If, therefore, we know the effects of the attraction of different bodies, together with their magnitudes, we can find their densities, and thence their quantities of matter."

And this is true, in case the theory of equal innate gravity of matter be true ; in which case, the density of a central body, as of Jupiter for instance, will be numerically expressed in the reciprocal of the product of the magnitude of the central body by the square of the period of the revolving body; which meth- 
od, if the principle were but correct, would furnish a very easy method of obtaining the different densities, (so called,) or the proportional quantities of matter of those planets having satellites revolving around them.

But the world are wishing for the best evidence by which to account for the phenomenon, that the power of a planet to attract its satellite varies in proportion to the magnitude of the planet, in the inverse ratio to its distance from the sun. And for the purpose of presenting some natural and consequential evidence upon the subject, I will present, in short, a method which I would pursue in the deduction of consequences from observed phenomena, with a view to some unity of purpose; and to this end, I would assume the distance of the moon from the earth to be unity or 1 . In which case, if the distance of the earth from the sun be $95,000,000$ miles, and the distance of the moon from the earth be 237,500 miles from the earth, (as estimated by some,) the proportional distances between the earth and moon, and between the earth and sun, will be as 1 to 400 , which I will assume for the sake of round numbers. And as the relative motion of the moon in its orbit about the earth, is to that of the motion of the earth in its orbit about the sun, as 1 to 29.9207, the square of which is 895.2428 , this multiplied by 400 , (the relative distance of the sun,) gives 358099 , and a fraction, as the relative power of the sun to that of the earth;that of the earth being called unity. But the exact proportion between the power of the earth and sun must depend upon getting the true relative distances between the earth and moon; and between the earth and sun, which, perhaps, cannot be obtained to a great degree of exactness.

So, if we take the mean between the estimates of Cassini and Sir Isaac Newton, as the mean distance of Jupiter's fourth satellite from Jupiter, its relative distance from Jupiter to that of Jupiter from the sun, will be as 1 to 427 , and the relative motion of such satellite in its orbit to that of Jupiter in its orbit, will be as 1 to 1.6463, - in which case the relative power of attraction of Jupiter to that of the sun would be as 1 to 1157.8501 . Hence, the relative power of attraction of the earth to that of Jupiter (calling the distance of the moon from the earth 237,500 miles; or rather, calling the relative distance of the moon from the earth to that of the earth from the sun as 1 to 400 ) would come out as 1 to a little more than 309 . Or, if we call the relative distance of the moon from the earth to that of the earth to the sun, as 1 to 391 , then upon the foregoing calculation, the relative force to that of Jupiter would be about as 1 to 312 , which is in general about the proportion assumed. But it must 
be manifest that the estimates of relative power must depend upon the relative distances we assume; and those cannot be ascertained to a very great degree of exactness.

The foregoing method goes upon the principle that the force exerted by the sun upon the planets is in the inverse ratio to their respective distances from the sun; and hence that the mean force exerted by the sun upon a planet is the square of the mean motion of the planet; and that such is the case in respect to the earth and moon, as in respect to Jupiter and its satellites. . And hence, that the force exerted by the sun upon the earth at the distance 400 is a little over 895 times as great as that exerted by the earth upon the moon at the distance 1 .

Thus the force exerted is always inversely as the distance; and hence the proportional power of gravity or attraction of the respective planets of the solar system, are as their respective magnitudes and their inverse distances conjointly. That is, if one planet be twice the distance of another planet from the sun, its power of attraction will be but just one half as great in proportion to its magnitude as that of the other.

And when inquiry into the phenomena of nature presents and submits itself directly to mathematical investigation and solution, corroborated by well known laws, - taking for data such elements only as would most naturally assign the true cause from the result produced, without seeking further for an hypothesis by which to account for the cause of the phenomena; we may feel much confidence in a conclusion which so naturally follows from such investigation.

Thus, the square of the mean motion of a revolving body. multiplied into its mean distance from the central body, gives the ratio of force between the two bodies. Or thus : if the relative motion of a planet compared with the relative motion of its satellite, be multiplied into the relative distance of the planet from the sun, compared with the distance of the satellite from the planet, we thereby obtain the relative power of the sun compared with that of the planet.

But as the mean motion of a planet $i s$ inversely as the square root of the mean distance from the sun, how the fact should have escaped observation, that the mean force is the square of the mean motion, and consequently inversely as the distance, I know not; for the world has certainly acknowledged those principles in the methods they have pursued for finding what they have conceived to be the different densities of the planets.

Thus, they make the mean motion of a satellite, the square root of the mean force of the planet, exerted upon the satellite; and so do I.

So if there were three planets, $\mathrm{A}, \mathrm{B}$, and $\mathrm{D}$, of equal magni- 
tudes, revolving about the sun, $\mathrm{A}$ at the distance $1, \mathrm{~B} 2$, and D 4 ; each having a satellite revolving around it at like distances, one with the other; in such case, by my calculation, as well as by that made by the world in their investigations, the attractive powers of the planets compared with each other, will be inversely as the squares of the periods of their respective satellites; and upon this ground the world have placed the comparative, or rather relative attracting powers of the earth and Jupiter; and so have I.

The consequence then is, that the reciprocal of the square of the distance of a satellite, divided by the square of the period of the satellite, gives the attractive power of the primary.

Then these being facts and results from all calculation, it only remains that the true cause should be assigned to the phenomena thus observed.

But whatever may be the cause why the planets exert a force; thus proportioned to their respective distances from the sun, upon their respective satellites, we do find that the force thus exerted, is the square of the motion produced in the satellite; thus emphatically determining the law of force to be inversely as the distance; and this goes far towards overturning that fortuitous notion, that matter, gross matter, contains, or is possessed within and of itself, all the requisite powers and principles for its government and control throughout eternity.

Thus do we find that not only is the force of gravity inversely as the distance; but that a primary planet only exerts a force upon its satellite in the inverse ratio to the distance of such primary from the sun; which fact I shall make use of as an unequivocal inference hereafter.

I have already intimated, that from the many analogies which magnetism bears to that of attraction in the solar system, it deserves consideration; especially in the analogy presented in the powers of attraction of the planets as proportioned to their respective bulks and distances from the sun; and from which analogies, much evidence is certainly derived to justify the hypothesis, that the sun is the great magnetizer of the bodies which compose the solar system; and that the primary planets possess no other attracting power than that communicated to them by the sun; and also, that the attracting power of the primary planets is in the inverse ratio to their respective distances from the sun, and their respective bulks or magnitudes conjointly; and that the density and intensity of what I will here call the magnetic atmosphere of the sun, varies inversely as the distance varies from the sun.

It could scarcely be expected from our present limited knowledge of magnetism, (even since the discoveries of Galvani, 
and Oerstead,) that we should be able to trace out all the analogies existing in its various forms and modifications;- or of solar magnetism, in all its various forms and modifications; but nevertheless, there would seem to be enough under all circumstances, to make it more than probable, that the laws of magnetism are precisely those of solar attraction.

Although we may well suppose the sun to be a permanent magnet, yet perhaps there are no direct means for determining whether the magnetism of the planets is permanent, or whether they are only dependent upon the sun for their magnetism, while lying within his magnetic influence, in the same manner as are particles of iron filings, which become magnetic and attract each other when brought within the influence of a permanent magnet; or like soft iron when under the influence of the electric or galvanic fluid; in which cases the magnetism ceases when the indueing fluid is withdrawn.

If then the attractive power of a planet is not an innate principle of matter, but is only induced by the magnetic influence of the sun or centre of attraction, in the manner in which iron filings are operated upon when under the magnetic influence, or soft iron, when operated upon by the Galvanic, or Electro-magnetic fluid; then a planet revolving in an eccentric orbit, would possess a greater power of attraction when in perihelion, than when in aphelion; and its increasing force and motion while passing from its aphelion to its perihelion, would tend to dilate the orbit of its satellite; and the reverse while proceeding from perihelion to aphelion; and were the orbit of such planet sufficiently eccentric for determining so nice a test, doubtless, the oscillations of a pendulum of a given length, or of a compass-needle, would be more rapid when the planet was in perihelion, than when in aphelion.

So likewise, if a soft piece of iron be made to revolve about a strong magnet, and within its magnetic influence, its power of attraction will vary in some inverse ratio to its distance; but I do not know but that it would be as dense in one part of its orbit as in another.

I think then there are analogies and unequivocal inferences in support of the hypothesis that attraction is not necessarily an innate or inherent principle of matter; but I know of no analogies to support the Newtonian hypothesis of universal gravity or attraction.

But perhaps. those analogies between magnetism and solar attraction, which may serve to explain, (at least, theoretically,) the more hidden or occult recesses of nature, depend upon the polarity of magnetism in all its various forms and modifications; and here we bring directly to our aid, the phenomena 
exhibited by electricity and Galvanism, which appear to be so intimately connected with magnetism as to induce the belief that they are but modifications of one and the same great law, but presenting somewhat different phenomena under different circumstances and modifications ; and that the magnetic fluid of the solar system is the medium in and through which all their various operations take place, either in the government of inert matter, or in the production of organic matter, and in the control of final causes.

Each of these modifications, (if such they be,) has its poles, or inverse influences, tending, nevertheless, to a junction with an ardency or intensity corresponding with the degree to which they appear to have been separated; which poles, or adverse influences, are so inverse in their operations, as often to perplex the mind in respect to them. Thus it is said, "what we call the north pole, (of the magnet,) because attracted by the north pole of the earth, is, for this very reason, the south pole of the magnet;" going upon the ground or well known fact, that poles of the same kind repel each other, while those of opposite kinds attract each otber; and why this circumstance has not given rise to a different method of investigating the operations of terrestrial magnetism, I know not.

I know it has generally been supposed that the earth was a great mass of inert matter; but at the same time possessed of power over other inert matter, by means of an innate or inherent principle of attraction; and that from some unaccountable circumstance attached to this mass of dead matter, it has two poles, a north and a south, with a magnetic equator, \&c. Some who have believed in the volcanic theory of internal fires, as connected with the Huttonian theory of geology, have been apprehensive lest those fires were not yet sufficiently exhausted, to prevent danger from a breaking through of the crust in some volcanic eruption, or violent earthquake. And many a curious calculation has been gone into, to show how long it would take a mass of matter as large as the earth, to finally cool through and become one solid mass.

Some have imagined that there was latent heat or caloric enough combined with the solid matter of the earth, to reduce the whole mass to what they conceive to be its primitive gasses, (should that heat once, become active,) and again fill a wondrous space with its rare and primitive elements; while others have been perplexed with the geology of the earth; the inequalities of its surface; the vast apparent changes that have been going on in time, at and near the surface; the vast regions that have manifestly been raised from under the waters of the ocean, even into elevated mountains, \&c.; many of which phenomena are 
truly wonderful when unaccounted for; and that they are satisfactorily accounted for by the causes which have generally been assigned, will not be contended for by a large portion of the community.

But had geologists, - while endeavoring to assign causes for those phenomena, in lieu of placing the adverse poles of the magnetic fluid, (in its various modifications,) upon the surface of the earth, where they appear to have but little efficiency in the great operations of nature, or but little use, except in directing the compass needle, - only placed them in contact, at the centre of the earth, they would at once have assigned a cause sufficient to account for all the phenomena that have so much perplexed them, by establishing a laboratory sufficient for all the internal operations of the earth; as well as to account for most of the geological phenomena upon and near its surface. The intensity of heat would then pervade the whole internal region of the earth, and fuse the whole internal mass. There would be the process of combining, separating, and mineralizing the various metals, minerals, and other substances, to the utmost capacity.

As certain portions of the solid parts of the earth became thinner, by the fusion of its inner parts, they would yield to internal pressure and gradually recede from the centre; while the thicker, and less fused portions, would gradually become central portions; until their inner portions in turn, should become molten, while other portions were cooling and becoming thicker; and by thus alternating, (but without much apparent uniformity,) would, in connection with the volcanic flues or vents to the internal fires, which occasionally and alternately pervade the surface of the earth for periods of time, cause all the phenomena upon the surface; as that of the inequalities upon its surface, geological strata, the veins of metals, \&c. This theory would most admirably account for the independent coal formations which are found to exist at and near the surface of the earth, more commonly in latitudes remote from the equator, and which are doubtless caused by deposits of vegetable matter, conveyed from towards the equator by ocean currents, as that of the Gulf-stream, for instance, which in process of time, under pressure and heat, is converted into coal, and thence raised above the ocean for the future use of man.

This gradual rising and falling of different portions of the crust, (and with such uniformity only as all the circumstances would permit, some perhaps being raised or sunk several times, while others were stationary, or nearly so, together with the alteration of the internal portions of the solid parts, by the action of heat, as well as by new volcanic vents produced, or old nnes 
closed, or opened anew,) would cause the magnetic or galvanic fluid to become somewhat varied in its direction; and thereby cause a corresponding variation in the magnetic needle. And should the magnetic meridians be found to correspond with the thicker or thinner parts of the crust, or with lines having reference to the great chains of volcanic vents, or to their greater or less activity; in such case, the declination of the needle would only serve to indicate where those sections were.

There are certainly strong indications that the magnetic (or galvanic) current, is in the direction from the equator (or rather the magnetic equator) of the earth, towards the external poles of the earth ; and that in all this distance, at least until we arrive at a high latitude, it is received and conducted by the surface of the earth, in a curve corresponding with the surface or nearly so; increasing in quantity or intensity as we proceed towards the higher latitudes; until the whole shall at length be conveyed, by this conducting curve, to the internal poles; and hence, from this constant accumulation in quantity, as we advance towards the higher latitudes, the accepted pole of the needle becomes more strongly attracted or more strongly inclined to correspond with the magnetic current; and hence, the oscillations of the needle in setling to its meridian will be more rapid as we approach the higher latitudes, than they are at the magnetic equator.

But it is by no means certain that the magnetic fluid becomes more abundant or intense up to 90 degrees of latitude; and if not, (and so the inference would seem to be, then, whenever the point of greatest intensity was arrived at, the dip or inclination of the needle, would be at 90 degrees, having lost all horizontal traverse or direction, and thence would remain in that situation or nearly so, up to 90 degrees of latitude; and hence, the compass-needle might become quite useless as a guide to the mariner in the highest latitudes.

But as the poles of magnetism, electricity, and galvanism, (which in their effects and operations appear to be but different modifications of the same agent or power,) are so inverse in their apparent operations, it behoves us to have a care, lest we assign to them effects that do not belong to them. Thus it is said, "what we call the north pole of the magnet, because attracted by the north pole of the earth, is, for this very reason, the south pole of the magnet."

Now the ground of this dictum, is the assumption that the external poles of the earth are the only actual and effective poles of the magnetic or galvanic fluid; and if such were the fact, the allegation in respect to the compass-needle or magnet, 
would be correct, could we but reconcile the idea with the dip or inclination of the needle. But aside of the little consequence that we are able to deduce from the external poles of the earth, (except that of giving direction to the compass-needle, and which, by the way, becomes less and less a direction as we advance into the high latitudes, antipodes as those external poles are to each other, - and so contrary to all experience, in producing effects by the adverse poles of the magnetic, electric, or galvanic fluids;) the inferences, would seem almost unequivocal, that the fluid of what we commonly term the north pole of the magnet, is identical with that at the external north pole of the earth; and that the error has arisen from supposing the external poles of the earth to be the actual poles of the fluid; and not that the earth formed a conducting circle from its surface to its centre; and that the pole which has been conceived to have its direction towards the north, has in fact, its direction towards the south, at the centre of the earth, where it meets the opposite pole, and where the battery performs its operations.

And hence, the fluid of either pole of the magnet, or needle, is always impelled in the line of direction of its own identical fluid; seeking connection or contact with the opposite pole at the centre of the earth; but when fixed, chained and iron bound to a steel bar, and not at liberty to pursue its wonted course, it is always ready to turn aside, and enjoy connection with the adverse fluid, whenever opportunity or inducement may offer; but such does not offer when fastened at opposite ends of the same straight bar; - and those subtle fluids never mistake in their object.

I will then assume for the fluid of the respective poles of the magnet, its wonted inclination in fact; viz. that of flowing in the current of its own identity when left free to do so; and hence, in the direction assumed by the compass needle.

I have said that the adverse currents flow all the way from the magnetic equator towards the external poles of the earth; and thence to their respective poles at the centre. This was only by way of exemplification; for there is much evidence to show (by way of inference at least) that the whole surface of the earth is both a receiver and conductor of the fluids for both poles; but that each end of the earth only conducts its respective fluid to the centre, or central poles. Thus, on all parts of the earth, so far as we know, artificial magnets can be formed possessing the adverse fluids, each being inclined to pass with the fluid of which it is identical.

If this theory in respect to magnetism and the earth, be true ; if it be sustained by all the analogies arising from experiments 
made with magnetism, electricity and galvanism, either singly or combined; if it be impossible otherwise to rationally account for many of the phenomena observed in geology, and especially that hypothesis so strongly inferred as to compel most people to implicitly believe the hypothesis a fact, and to endeavor to account for it by the most vague, absurd, and unknown cause, viz. the central fires of the earth; - then let us adopt the theory, and transfer all the analogies to the other primary planets, and to the sun; all of which, being governed by the same great laws of nature as the earth is, especially by that of attraction, or the great physical law of the universe, apparently require internal fires as much as the earth does; and also that they should be made in the same way; and if beneficial, that they should be continued, and not liable to be extinguished, nor to frighten those mortals who inhabit the surface, nor put them in danger of falling into them by breaking through the crust.

Conceive then that the sun is the centre of our system; and that it is surrounded, to an unknown extent, by what we must probably conceive to be a highly attenuated and subtle fluid, which is the medium of attraction; and that this fluid, or medium, becomes less dense or less intense in proportion to the distance, or as we recede from the sun;

That by and through this medium of attraction, the sun becomes the magnetizer of all gross matter in the solar system; in which case, the primary planets are magnetized in the proportion to their inverse distance from the sun; thereby giving them attractive powers in proportion to their bulks directly and their inverse distance conjointly; and hence their inverse distances from the sun denote their respective rates of gravity towards the sun;

That each planet, from being thus magnetized by the sun, becomes itself imbued by a portion of the magnetic fluid, the density of which is proportioned to the bulk of the planet directly and its inverse distance from the sun conjointly; and hence, its power to attract other matter; and in such case, it would be hard to conceive that the magnetic atmosphere of the sun would be a resisting medium to the planet, from the circumstance that the fluid as readily attaches to the planet as to the sun; in which case, all resistance to the motion of the planet would be annihilated far in advance;

That the sun and planets are supplied with internal fires in the same manner that the earth is; and that too in proportion to their respective surfaces and their powers of attraction; and also with volcanic flues proportional, as outlets to such internal fires; That neither light nor attraction, or gravitation, are emana- 
tions from matter, in the sense in which they have generally been considered; that neither of them are progressive in time; but are instantaneous in their effects at all distance subject to their effects ; and hence, that the effect, or rate of intensity of each, is inversely as the distance; nor has light anything to do with filling space with particles, or rays of light, as has in general been taught and understood;

That the atmospheres of the sun and planets, properly so called, (viz. that which consists of the gasses, gross vapors, and impalpable matter that surrounds those bodies,) may well be conceived to have some near proportion to the size, and to the power of attraction of the bodies which they surround; the density of such atmosphere depending upon its elasticity and the power by which it is attracted, must necessarily depend on the amount of matter which it contains, and the power of attraction with which it is drawn towards the body which it surrounds; and hence its capability to affect light by refraction, reflection or otherwise; and hence its power for raising gross vapors from the body which it surrounds; which in case of the sun, must be with great rapidity, and to a great height;

That when we view the sun through a telescope, we see its solid parts; but through an extremely dense and brilliant atmosphere of vast extent;

That the spots often observed upon the sun are caused by volcanic eruptions, or discharges of fire, smoke, vapors and other substances from the vents to the internal fires; which, rising in vast quantities, with great rapidity, and to a great height, often spread the umbra to a vast extent, so as to hide large portions of the sun's surface from our view; and while the eruption continues, the boundary between the nucleus, or matter immediately ejected, and the umbra, will be well defined; and also, that the nucleus will naturally vanish before the umbra; and on their both vanishing, a red hot lava may for a time present to view what is termed a facula, until it shall at length take the appearance of other parts of the surface of the sun.

If, however, the sun, or centre of the solar system, be surrounded by a vast magnetic atmosphere of ever so great tenuity; if the same vary in density in the inverse ratio of the distance, and is sufficiently dense to refract light, (the central battery of which fluid is at the centre of the sun,) the same would most ad. mirably account for the phenomena observed by Dr. Bradley, from which he deduced his hypothesis of the progressive motion of light in time, as also for the zodiacal light. Notwithstanding, La Place would reject the last suggestion, for the reason that it would interfere with the Newtonian theory of gravity, which he has so liberally endorsed to the world. 


\section{H A P T E R VI.}

\section{On the Tides.}

IN accounting for the tides upon the principle of the attractive powers of the sun and moon upon the earth, and the waters of the earth; those who have attempted it, have assumed as a lemma, or at least an hypothesis, that the force of gravity is inversely as the square of the distance; and as the apparent diameter of the moon is to that of the sun when viewed from the earth, as 1.091 to 1 , and as according to their law of gravity, the respective influences of the sun and moon upon the earth, should be as their apparent diameters, in case their densities were equal, hence, according to their apparent diameters, the moon would have but very little more influence upon the tides than the sun has.

But finding that the greater part of the tides are caused by the influence of the moon, it has been concluded that the moon is much more dense than the sun; which densities as proportioned to each other, they have weighed and determined, so far at least as to satisfy themselves and many others, by resorting to a comparison of their supposed respective influences upon the tides; which, to be sure, has cost them no small trouble; nor have their conclusions been very uniform or satisfactory, even to themselves.

Bernoulli estimates the influence of the moon to that of the sun upon the tides, as 2.5 to 1 ; Newton, as 3.5 to 1 ; and others have even estimated it as 16.7 th to 1 ; La Lande, as $27-10$ ths to 1 ; and La Place 3 to 1 . But Bernoulli's ratio is in general settled upon, as being thought the mo'st probable. Hence the conclusion, that if the joint influence of the sun and moon will raise the tides 7.445 feet, the moon's portion will be 5.412 feet, while that of the sun will be 2.033 feet.

But after all, they seem to labor under difficulties in adapting their theory to observation; for Mr. Vince, after enumerating many difficulties of this kind, observes, "Thus the theory alone will afford no practical conclusions." And in justification of 
certain tables based or founded on theory,-after consenting that they will not agree with observation, - says: "To determine, therefore, the true time at any port, we must find from observation what is the difference between the true time and that shown by the table, and then that difference added to the time shown by the table will give the time of the high tide."

In the American Encyclopædia or Conversations Lexicon, the reader, under the article Tides, will find a somewhat racy exposition of the commonly received theory of the tides, and as the articles in that work, though often giving but an epitome of the subjects of which they treat, are nevertheless written with ability, giving the most approved philosophy of the day in which they were written, - as is the case with the article alluded to, which is an article also well calculated to satisfy the minds of most people upon a subject of which they had thought and cared but little, and who were not very curious to know if these things were so, - and as it is somewhat convenient to have it here inserted, with a view to commentary and comparison, I will make a liberal extract from the same, notwithstanding the work itself is readily accessible to most readers.

"The ebb and flow of the sea are evidently connected with the moon's motions. The level of the ocean is slightly disturbed by the attraction which is alternately exerted and withdrawn. The waters for a large space under the moon, being more attracted than the great body of the earth, are thus rendered lighter than those parts of the ocean which are at the same distance as the earth's centre, and being lighter, they are forced upwards a little by the surrounding mass, which is heavier, just as water and oil will stand at different heights in the two branches of a syphon tube ; or just as ice, which is lighter than water, is made to rise a little higher on that account, when placed in water.

"If the earth rested immovable upon a fixed support, there would be a tide or rising of the waters only on the side towards the moon. But the great body of the earth is just as free to move as a single particle of the ocean, and if suffered to yield to the moon's attraction, would be carried just as fast. Hence for the same reason that a particle of water, on the side of the earth towards the moon, is drawn away from the centre, or has its downward tendency diminished, so the solid earth itself is drawn away from the mass of waters on the side of the earth farthest from the moon. It is the difference of attraction in both cases between the surface and the centre, which causes the lightness of the waters and the consequent elevation. It will be seen, therefore, that, taking the whole earth into view, there are always two high tides diametrically opposite to each other, and 
two low tides also midway between the high ones. The high tides are two great waves, or swells, of small height, but extending each way through half a right angle. These waves follow the moon in its monthly motion round the earth, while the earth, turning on its axis, causes any given place to pass through each of these swells and the intervening depressions in a lunar day; or twenty-four hours and fifty minutes. What we have said with respect to the moon's influence in disturbing the level of the ocean, may be also applied to that of the sun; only in the case of the sun, although its absolute action is about double that of the moon, yet on account of its very great distance, its relative action upon the surface of the earth compared with that at the centre, is but about one third as great as that of the moon. At the new and full moon, when the sun's and moon's action conspires, the tides are highest, and are called spring tides. But at the first and last quarters of the moon, the action of one body tends to counteract that of the other; and the tides, both at ebb and flow, are called neap tides." "That the high tide when the moon is above the horizon, exceeds the high tide when the moon is below the horizon. And that $50^{\circ}$ towards the nearest pole from where the moon is vertical, there will be only one tide in twenty-four hours," \&c.

Now the theory contained in this running account of the phenomena of the tides, (though it were the result of La Place's greatest intellectual effort,) is not calculated to give full satisfaction upon the subject.

1st. 'The waters on the side of the earth towards the moon, are more attracted than the great body of the earth, - made lighter than the waters at the distance of the centre of the earth, - and hence forced up by the surrounding mass of waters.

$2 \mathrm{~d}$. If the earth were made immovable, (viz., so that its centre and solid parts could not be attracted towards the moon,) there would be a tide only on the side of the earth towards the moon. But as it is not fixed, the solid earth is drawn from the mass of waters on the side of the earth opposite the moon, and hence, creating a tide on the side of the earth opposite the moon.

$3 \mathrm{~d}$. That the tides on both sides of the earth are caused by the difference of attraction between the surface and centre of the earth.

4 th. That the high tides are two great waves or swells, extending each way through half a right angle, following the moon in its monthly motion round the earth.

5th. That the sun has a like influence upon the waters that the moon has, - only, though the sun has about twice the absolute action upon the earth that the moon has, yet from its great 
distance, its relative action upon the surface of the earth compared with that at the centre, is but about one third as great as that of the moon.

In the foregoing theory there is certainly an ample number of causes assigned to produce the effect or phenomena, in case they are such as are sufficient. Thus, if the attraction of the moon makes the waters on the side of the earth towards the moon lighter than they are at the distance of the centre of the earth from the moon, so that they are forced upward by the heavier surrounding waters, this cause would be sufficient for causing the tide on the side of the earth towards the moon; and would seem to dispense with that of their being attracted away from the central parts of the earth. But as the surrounding waters could only press those upward which were made lighter on that side of the earth towards the moon, this cause alone could not, therefore, account for the phenomena of the opposite tide; and hence, inasmuch as the opposite tide must be accounted for from some other cause, and by one which would as well account for both as for either, the whole cause seems rather at length to be assigned to that of the waters on the side of the earth towards the moon being attracted from the central parts of the earth; and the central parts of the earth being attracted and drawn from the waters on the opposite side of the earth.

This doubtless goes upon the ground, that if the earth were an entire mass of waters, and were not disturbed by any foreign power, the mass would be in shape of a perfect sphere; but if attracted by the moon, (for instance,) it would become elongated; its greatest diameter being directed towards the moon, - in like manner as Sir Isaac Newton conceives the moon to be in respect to the earth; with this difference, however, that while Newton supposed the end of the moon which hung next to the earth was heavier than the other end, the contrary would be the case in respect to the ends of the earth.

But we will suppose the internal parts of the earth to be solid, or composed of solid matter, having its surface covered with deep waters; and then suppose the tides to be two broad waves directly opposite each other; that the one nearest the moon shall be caused by being attracted from the solid parts of the earth, and the opposite one shall be caused by the solid parts of the earth being attracted from the opposite waters; the high water of that wave nearest the moon being, however, some three hours or $45^{\circ}$ behind the moon, (or the position of the moon, caused by the diurnal motion of the earth;) and then let us endeavor to calculate the motion of the solid parts of the earth, (as well as of 
those two great waves) caused by the disturbing force of the moon.

Will it be said in such case, that the solid parts of the earth, in consequence of the attraction of the moon, make or perform a revolution in an orbit, in about twenty-four hours and fifty minutes; the direction of which orbit will be subject also to all the wanderings, or northings and southings of the moon; and that the direction of the solid parts of the earth in such orbit is always towards the point of high tide, in that wave or swell nearest the moon?

If so, to calculate and adjust the equations of the earth's annual orbit, required in consequence of this disturbing force, would puzzle even a La Place.

But it is said the sun has about twice the influence upon the earth that the moon has, but from its very great distance, its relative influence upon the surface of the earth, is but about one third as great as that of the moon compared with that at the centre. Hence, according to this hypothesis, the influence of the sun upon the centre of the earth, must be vastly greater than that of the moon; and I will here merely query, how those joint, opposing, or varied influences of the sun and moon, are to affect the centre and surface of the earth, in all the wanderings of the moon; how in conjunction, in opposition, in quadratures, \&c.

To me, the whole theory is but a tissue of absurdities and dilemmas; as though cause and effect had been guessed off, without much consideration with regard to their connection, mutuality, or reciprocity; and as if the connecting links had been but little regarded.

Nor does the foregoing theory differ essentially from that of Mr. Vince. Mr. Vince, - after observing that Kepler was the first who assigned the true physical cause of the tides, namely, "that the waters of the sea gravitate towards the moon, and cause the tides;" and that Sir Isaac Newton had shoven that from the principles of gravity, the phenomena of the tides may be solved, - proceeds with an attempted exposition of the phenomena, upon the Newtonian principles, by referring to the supposed operation of corpuscles, of infinitely small magnitudes, upon each other, (by which the world has been so much darkened , in its researches after truth;) and after alleging difficulties that constantly prevent fact from agreeing with theory, as for instance, "that the free motion of the waters in the open seas is hindered by shallow places, rocks, and islands, in consequence of which, the tides in some of the open seas, at the time of the conjunction of the luminaries, are found to rise only to the height of about 
three feet," proceeds to remark thus, in which the case seems rather to be summed up:

"The general phenomena of the tides from observation, agree very well with the conclusions deduced from the theory of gravity, indeed much more accurately than could have been expected, when we consider how many circumstances there are which take place, and which cannot be reduced to computation. The theory supposes the whole surface of the earth to be covered with deep waters, that there is no inertia of the waters, that the major axis of the spheroid is constantly directed to the moon, and that there is an equilibrium of all the parts. But the inertia of the waters will make them continue to rise after they have passed the moon, although the action of the moon begins to decrease, and they come to their greatest altitude in the open seas about three hours after, at which time there is not a general equilibrium, but the waters rise and fall by a reciprocation; hence, the longest axis is not directed to the moon, nor is the figure a perfect spheroid. 'The waters have not a free motion on account of the shallow places, rocks, islands, and continents, the force of currents and winds; also as the waters approach the equator where the earth has a greater velocity about its axis, they must necessarily be left behind, and obstruct the regular motion of the water when it moves from west to east, but conspire with that from east to west.

"All these circumstances must affect the measures of the phenomena as deduced from theory; it may, however, in many cases, give the relative measures without any great error, so that by accurate observations once made, on their absolute quantity, in some one particular case, the measures, in all other cases, may be ascertained to a considerable degree of accuracy."

Now, from the phenomena of the tides, as found from observation, perhaps no one would doubt Kepler's hypothesis, that they were caused by the influence of the moon; and had not Newton and Bernoulli subterfuged and perplexed the theory, in Newton's mysterious law of gravity, in connection with the ease and facility with which La Place has been able to equate the effects of all disturbing forces that might be suggested, it is highly probable that the world, from observation, would long ago, have come to more rational conclusions as to the manner in which the influence of the moon is exerted in causing the tides.

Mr. Vince, who always treats general subjects with the utmost candor, seems rather cautiously to have avoided descanting much upon the cause of the tide on the side of the earth opposite the moon; and hence, has left this part of the subject rather destitute of explanation.

I have thus presented some of the palpable and insurmount- 
able difficulties under which the popular theories labor in attempting to account for the phenomena of the tides upon the Newtonian law of gravity, and more especially upon the Newtonian hypothesis of universal gravity, which; in my humble opinion, lays the whole foundation of the fabric of astronomy in fortuity and atheism. And if, as is so whimsically alleged, for the purpose of giving it a marvellous effect, this hypothesis was the offspring of the fall of an apple, the consequences of that fall, (comparing small things with great,) were not less deleterious to the scientific world, than was the forbidden fruit to the moral world.

I will now, after a few preliminary remarks upon the law of gravitation according to my determination, (viz., that the force of gravity varies inversely as the distance varies,) give an outline of my theory of the tides, based upon my theory of universal gravity as connected with my theory of the solar system.

By my determination of the lave of gravity, if the moon were to revolve about the earth in an orbit but 1-4th the distance that it now does, its influence upon the earth would be four times what it now is, and by the Newtonian law of gravity, its influence would be sixteen times what it now is. But according to my theory of the tides, I should, according to my law of gravity, make its influence upon the tides a little more than four times what it now is ; and by the Newtonian law of gravity, I should make its influence upon the tides a little more than sixteen times what it now is; for the motion of the moon in its orbit must of necessity, be just double what it now is, if it revolve four times as near the earth; and hence, the motion of the moon in its orbit being doubled, its apparent motion over the surface of the earth, caused by the diurnal motion of the earth upon its axis, would not be quite as great as it now is. But whether upon the popular theories of the tides, what are conceived to be the great tidal waves of the ocean would swell to four times the height in the one case, or to sixteen times the height, in the other case, that they are now conceived to swell, I will not undertake to determine.

The estimated bulk or magnitude of the moon to that of the earth (which is doubtless correct) is as 1 to 49 , and if their influences or attractive powers upon each other are in proportion to their respective bulks or magnitudes, (and from all analogy as yet deduced from observation, there is little or no doubt of the fact, ) their respective influences upon each other will be as 1 to 49 .

If we now take round numbers, and consider the distance of the moon from the earth to that of the earth from the sun as in the proportion of 1 to 400 , - which is in accordance with the estimation of some, and is sufficiently near for our present purposes, 
although it makes the distance of the moon somewhat less than the common estimate, if we call the distance of the sun $95,000,000$ miles from the earth,- in such case, according to my determination of the law of gravity, the attractive power of the sun upon the earth or moon would be some eight hundred and ninety-five times as great as that of the attractive power of the earth upon the moon; as denoted and determined by the motion of the earth in its orbit about the sun, and the motion of the moon in its orbit about the earth ; and consequently, that the attractive power of the sun upon the earth would be more than forty thousand times as great as that of the moon upon the earth.

And, yet, according to my theory of the tides, no portion of them are caused by the direct influence of the sun upon the earth, or upon its waters; although its indirect influence exerted through the moon, causes the variation from spring to neap tides. And in fact, if my determination of the law of gravity be correct, it is quite certain that the influence of the sun upon the tides compared with that of the moon (whether direct or indirect) must be exceedingly small, when compared with their respective attractive powers upon the earth.

But I will conclude my investigations of the theories extant, in the words of Mr. Vince, in which he again sets forth, by way of repetition or recapitulation, the difficulties to be encountered under present theories; and will thence proceed to a theory of my own. After many attempted explanations of the causes and phenomena of the tides upon the Newtonian theory of gravity, he says, "It has been here supposed, that the high tide was under the luminary, and that there was a general equilibrium of the waters; but the high tide is at some distance from the lumi. nary, and the waters rise and fall by a reciprocation; also the free motion of the waters in the open seas is hindered by shallow places, rocks and islands; in consequence of which, the tides in some of the open seas, at the time of the conjunction of the luminaries, are found to rise only to the height of about three feet," and concludes by saying, "thus the theory alone will afford no practical conclusions."

So much for Mr. Vince, and other authorities upon the theory of the tides.

I will now present a short outline of my own theory of the tides, based upon my theory of universal gravitation; and upon my theory of the solar system, submitting it to the consideration of an inquiring world, who have ever been anxious, from the days of Aristotle and Virgil, to know

Why flowing tides prevail upon the main,

And in what dark recess they shrink again. 
Malte Brun, who was somewhat of a whimsical and credulous philosopher, (however eminent he was as a geographer,) treats of the tides mostly after the popular methods; he has some remarks, however, not unworthy of notice, as "that they have tides in the islands of the South Sea of only one or two feet elevation, whilst upon the western coasts of Europe and the eastern coasts of Asia the tides are extremely strong and subject to many variations." Now Europe and Asia make a very broad continent; and the western shores of Europe and the eastern shores of Asia are washed by broad oceans.

The same author says, "In the torrid zone, the flood-tides run from east to west with the motion of the stars," but he says nothing as to the direction of the ebb of such tides, and might leave us to infer that the tides in the torrid zone may have some connection with those ocean currents with which he has made such a display, that it has been thought prudent to suggest, that he may have accounted for more than existed.

Such ocean currents, however, as are known to exist, and which have been traced, or the courses of which have been ascertained, seem very well calculated to aid and corroborate a true theory of the tides; nor do I conceive it a matter so inexplicable as the author above named would intimate, that a current should proceed from the Ethiopian ocean to the coasts of Brazil, - that a current should proceed from the coast of Brazil and extend towards the eastern coasts of America, and prevail between $30^{\circ}$ north, and $10^{\circ}$ south latitude; and that within the above limits (of the degrees of latitude no doubt) there should be a current running the contrary way, or toward the coast of Africa. These currents, however, all seem inclined to run towards continents, or large tracts of land, and thence to float along the shores or near them, and to pass strongly through straits, or narrow passes, as between New Holland and Van Diemen's Land, \&c. But few of the ocean currents, however, have been very distinctly traced; nor will I do more than merely to suggest or hint at a subject so little known or understood, in corroboration of my theory of the tides.

\section{SECTION SECOND.-THEORY OF THE TIDES.}

My theory is, that the tides are caused by the direct influence of the moon, the sun exerting no other influence in raising the tides than an indirect one exercised through the moon; although the attractive influence of the sun upon the earth is many thousand times greater than that of the moon upon the earth; 
That the earth possesses two fixed poles in respect to the sun, which are susceptive of, and obedient to solar magnetism, (or the magnetic fluid of the solar system,) in conducting the same to the centre of the earth, for the purposes designed; which solar magnetism so wholly and powerfully pervades the whole earth, that solar magnetism is not perceptible in one part of the earth more than in another, if we except the stronger influence that may exist at the poles; but that the earth has no movable poles in respect to the sun;

That the moon has but one fixed pole in respect to the earth, and none in respect to the sun; but that its poles in respect to the sun, are movable and revolving; one always being towards the sun, and the other opposite to the sun. And that similar to this, is the earth in respect to the moon, so far as the magnetic influence of the moon is capable of creating polarity in the earth; the poles of the earth created by the moon being movable or revolving poles, one always being directed towards the moon, and the other in the opposite direction. And although those poles may be said to have some influence over the whole of both hemispheres of the earth, yet the greatest attractive power of those poles would always be directly under, and opposite to the moon, at which points matter would always be inclined to accumulate.

That the moon has but one fixed pole in respect to the earth, may fairly be inferred from the circumstance that one and the same side of the moon is always retained towards the earth. A circumstance which Newton attempted to account for upon the hypothesis that the moon might be elongated, and that, consequently, one end would hang towards the earth, while others have as gravely supposed, that one side of the moon might be heavier than the other. If, however, our present conceptions of polarity and magnetism will not permit the idea of a fixed or permanent pole without its opposite, there surely could be no objection to conceiving the moon to have two fixed poles in respect to the earth, both of them situated on the side of the moon next to the earth (exercising their influence in and throughout the moon,) any more than to a magnet having both its poles in one direction.

If the foregoing hypothesis in respect to the magnetic or attractive influences of the earth, sun, and moon, upon each other, be adopted, the cause and phenomena of the tides are readily accounted for.

In such case, the movable poles of the earth created by the moon, would be constantly tending to attract matter toward those poles; and should we conceive the earth composed of a fluid, as of water,- or to be covered with deep waters, and that 
one side of it is stationary towards the moon, for any considerable length of time, we might well suppose the earih would become elongated in the direction towards, and from the moon, to the extent of the moon's polarizing influence.

But if the whole globe were formed of water, or if it were covered with deep waters, and were to revolve upon its axis, as it now does, it may be hard to conceive how a tide could well be formed; for notwithstanding the earth would be polarized in a direction to and from the moon, yet, as those poles must travel on the surface of the earth, at the rate of about one thousand miles an hour, in consequence of the diurnal motion of the earth, there would be no time for the accumulation of the waters at those moving poles; and hence the waters must remain at rest, without rise, or without reciprocation of rise and fall. Nor can I conceive how the most imaginative or fanciful theorist, could, under those circumstances, assign the place where the waters should rise, or the reciprocation occur.

But the surface of the earth being divided into oceans and continents, and the matter upon the surface of the earth having a constant tendency (caused by the moon) to accumulate at the poles; hence, when the moon is passing over a broad continent and vast solid parts of the surface are under the influence of this polarization for a long time, or for several hours, the continent being itself fixed and immovable upon the surface of the earth, serves to produce an effect similar to that which would be produced, if the whole surface were covered with deep waters, and if one side of the earth should be made to remain stationary under the moon for hours together.

That is, the waters of the surface of the oceans adjoining the continent over which the moon should be passing, (and like phenomena would occur on the side of the earth opposite the moon,) would flow or tend towards such polarized continent, and pile upon its shores, ascend its rivers, \&c.

And hence, the flowing of the tides is always towards continents or large bodies of land, caused by the fixedness of the land, and its polarization by the moon; and the height of the tide, or the piling of the waters upon the shores, will be mostly governed by the magnitude or breadth of the continent, and the situation of the moon in respect to the continent.

So also the flowing of the tide may be east, west, south ot north, or in any direction, as the body of land shall dictate; and as the flowing of the tide is towards the land, its $e b b$ is necessarily from the land; and hence the rising and falling of what is termed the great tidal wave, by reciprocation, seems to savor more of fancy than of possibility or reality. 
Upon this hypothesis, the waters would rise upon the shores of islands near continents, the same as upon the shores of the continents; but in the middle of broad oceans, or far from large bodies of land, the tides would be small; so also on the shores of continents, where the continent was narrow, \&c.

While this hypothesis accounts for the tides being greater on the shores of continents than in the broad oceans, it also accounts for the reason of the tides not being so long behind the meridian of the moon, in the midst of broad oceans, as upon the shores of continents; as well as why it is behind the moon's meridian at all; without resorting for a reason, to the tardiness of the water in obeying the power of attraction, arising from its inertia, for the water will continue to pile upon the shores of a continent, so long as the continent, from polarization, possesses an attracting influence sufficient to draw the waters towards it; and it will not be denied, that time is requisite for the flowing of water, whether it be in a river or upon the surface of the ocean.

Should it be objected, that the time of high water on the west side of a continent, should not be so long behind the meridian of the moon as on the east side, I will only remark, that the whole cause of the tide arises from the influence which polarized terra firma has upon that substance, (namely water,) which is always at liberty to flow towards the fixed earth, as the moon, from its apparent rapid motion, cannot of itself move the waters at all. And each side of the continent is under the influence of the moon for an equal length of time; and most clearly, such objection would apply with equal, if not greater force to any other theory extant.

When the moon is in conjunction or in opposition, namely, in syzygies, the tides are highest; and when in quadratures, the tides are lowest, or very nearly at those times, the altitude of the tides at quadratures to those at syzygies being proportioned to each about as 1 to 1.3756 , although it is said the ratio will vary, from many circumstances. The tides also increase in altitude from quadratures to syzygies, and diminish from syzygies to quadratures; which increase has been attributed to the attraction of the sun conspiring with the moon at those times, and that the decrease is occasioned by the attraction of the sun, conspiring against the moon, or not in the line of direction with the moon; and hence, that the sun is gradually abstracting, or separating its own tides from those of the moon, as the moon approaches its quadratures; at which times the respective tides of the sun and moon are in quadratures, which must necessarily throw the earth into the shape of a double oblate spheroid, the 33 
altitude of the moon's tides being to those of the sun's as 2.662 to 1 , or as 5.412 feet to 2.033 feet.

That those four tides may exist when the moon is in quadratures, (according to the Newtonian theory,) both the sun and moon must draw away the solid parts of the earth from the mass of waters on the side of the earth opposite the given luminary; and hence, the motion of the solid parts of the earth could not be in a direct line toward either, (and whether an equation has ever been resorted to in consequence of those directing forces, I know not.)

So when the sun and moon are in conjunction, their united influence must draw the solid parts of the earth directly towards the sun and moon, leaving the mass of waters on the opposite side.

But when the sun and moon are in opposition, it may be hard to determine which way the solid parts of the earth will be drawn, as the tides would be equal under each luminary.

This then, must be a very perplexed question; nor do I think it in anywise solved, or the difficulty obviated, by the more modern and supposed ingenious theory of La Place.

For according to some authors, the sun exerts about twice the influence upon the earth, taking both its external and internal parts, that the moon does ; "but from its very great distance," it does not exert so much upon the surface. But in this case, it would seem that it might exert its whole influence fairly, as it is the internal and solid parts of the earth that are to be drawn away from the opposite waters.

It is alleged by some, that as the moon subtends a greater angle than the sun does, when viewed from the earth, its influence upon the earth is greater than that of the sun; and having concluded, from the amount of tide raised by each, (having no other method whereby to ascertain the fact,) that the density of the moon to that of the sun is about as 2.5 to 1 , and hence, that the difference between their attractive influences upon the earth where their respective subtended angles and densities are compounded, is about as 2.662 to 1 , by which it would seem that the moon has quite the advantage over the sun in its attractive influence upon the earth.

But to me all the theories extant in respect to the tides seem to be fraught with inexplicable dilemmas. I will therefore leave them, and will return and finish my own.

If then the sun exerts an influence on the moon wholly regardless of the fixed pole or poles of the moon, and similar to that exerted by the moon upon the earth, causing two moving or revolving poles of the moon, always directed to and from the 
sun, performing their revolution in the same time that the moon is revolving about the earth, or in revolving upon its axis in respect to the sun; then, when the sun and moon are either in conjunction, or in opposition, one of the movable poles of the moon will be extended directly towards the earth, in which case its full influence will be exerted in connection with the fixed pole of the moon, upon the earth, in increasing the influence of its movable poles; and hence, an increase of the tides. But as the moon proceeds towards quadratures, the influence of the movable pole of the moon upon the earth becomes gradually abstracted, as the line of its direction varies more and more from the earth, until quadratures; after which, the 'opposite movable pole of the moon commences and increases in influence until syzygies; and so on, alternating from syzygies to quadratures, and from quadratures to syzygies. And hence, no portion of the tides is caused directly by the sun, nor in fact, by the moon; their influence being only exerted in polarizing the earth; which polarization can only have an effect in raising the tides when situated in a continent, or terra firma, against which the flowing waters may pile:

This theory then, relieves from all disturbing forces incident to other theories of the tides; from the hypothesis that the earth is constantly drawn from a true curve, and hence performing its revolution in wholly an inexplicable orbit; performing "cycles and epicycles" in consequence of the attraction of the moon; and even from being disturbed in its regular course by any disturbing force of the sun. 


\section{CHAPTER VII.}

\section{An Investigation of the Theory of the Progressive Motion of Light.}

Perhaps neither the conjecture of Cassini the elder, (that acute observer in astronomy,) that light was progressive in time, from observations by him made on Jupiter's first satellite, (which conjecture, however, he shortly abandoned,) nor the supposition of Roemer from a like cause, that light is progressive in time, would ever have been adopted into philosophy as an infallible truth, and been the cause of so much time and trouble in endeavoring to equate the time in which light is conceived to be passing, had not Dr. Bradley, (astronomer royal to George II.,) some few years after the supposed discovery by Roemer, come to the conclusion of a progressive motion of light, from being unable otherwise to account for an apparent motion in the fixed stars, some of which, he had been very critically and carefully examining with a view to ascertain whether they had an apparent parallax or motion. From these observations he came to the conclusion, (which the world have ever since assented to, ) that light had a progressive motion in time; and that its velocity could be determined by his method, with far greater accuracy and certainty than could be hoped for or expected from observations made upon Jupiter's satellites. Since this time, in adapting equations, (even in respect to Jupiter's satellites,) recourse has been had to Dr. Bradley's hypothesis in making those equations, so far as the motion of light is to be considered.

But there are many reasons or causes why Jupiter's satellites furnish no conclusive evidence of the progressive motion of light, which naturally fall out from those astronomers who have most observed the satellites with a view to furnish correct tables of their eclipses, for the purpose of aiding the science of astronomy, and for the better ascertaining or finding of longitude. And it is even possible that from the equations of light which they have been led to adopt, they have also been led, for the purpose of forming correct tables, to adopt other and even em- 
pyric equations, which otherwise would have been dispensed with. Some of the causes why Jupiter's satellites would not be a safe criterion whereby to calculate the progressive motion of light, I will here recount.

1. The various modes or methods of ascertaining a synodic revolution of the satellite, as by an eclipse; the passage of the satellite over Jupiter's disk, \&c., which may lead to more or less error.

2. In an eclipse, the satellite does not always pass through the centre of Jupiter's shadow; hence from this cause some eclipses are longer than others.

3. Jupiter is a spheroid, and its shadow an ellipse; and the position of its shadow is not always the same in respect to the satellite.

4. The immersions are observed before Jupiter's opposition, and the emersions after; and those must be compared with each other at distant times in order to get their mean, or the true conjunction of the satellite.

5. Some of the satellites are said to be longer at some times than at others in passing through the centre of Jupiter's shadow, on account of the eccentricity of their orbits; and that the duration of eclipses are unequal.

6. It is said that from the unequal motion of Jupiter, the true conjunctions of the satellites compared with the mean may vary by twice the greatest equation of Jupiter's orbit, which amounts to near forty minutes in the first satellite, and more than an hour in the second. And on this equation a mean synodic revolution as well as the eclipses must depend. And even this equation must be variable, for the reason that the eccentricity and greatest equation of Jupiter's orbit is subject to change.

7. After making the equations for the supposed motion of light, a number of empyric equations are necessary in order to make the tables and the theory agree with observation.

8. Maraldi, whose tables have been esteemed, observed that even Cassini's tables erred, as to the time of the fourth satellite, near two hours, for which error several astronomers have attempted to assign a cause, as the eccentricity of its orbit, the situation of Jupiter in its orbit, \&c.

9. Error of time in calculating the entry of the satellite into the shadow, or upon his disk; and that the calculations deduced therefrom are different by different observers, and said not to be depended upon.

10. The first satellite is said by astronomers to be the best for finding the longitude; the tables of that being more correct, on account of its vanishing and appearing more instantly, in an eclipse, than the others, and therefore being more certain. 
11. Astronomers say it is better to compare eclipses observed under different meridians, than to depend on the tables, on account of their incorrectness; and then, that the observer should have the same kind of telescope.

12. Astronomers say, the time when a satellite becomes visible or invisible, depends upon many circumstances; as the telescope - the proximity of the satellite to Jupiter - the altitude above the horizon - the state of the weather, \&c.

13. Dr. Bradley found from observations made in Cassini's time, that the eclipses of Jupiter's first satellite lasted six minutes longer at some times than at others, which indicates that its motion was not uniform; but Baille and some others concluded it arose from the disturbing force of some of the other satellites.

14. Authorities say, that the satellite will disappear later and appear earlier, the better the telescope is. And some have observed, that the disappearance of the satellite depended on the distance of Jupiter from the earth and sun; and others have observed, that different states of the atmosphere, different altitudes, and their distance from Jupiter, have a like influence.

15. The authorities also say, that the time when the satellite becomes invisible at an immersion, or visible at an emersion, depends upon the quantity of light, which the telescope receives, and its magnifying power, as well as on other causes, - as upon the altitude of Jupiter above the horizon, the twilight, the clearness of the air, the proximity of Jupiter to the moon, and the eye of the observer; all combining to affect the time at which the satellite becomes visible or invisible.

It is highly probable that Cassini doubted the correctness of his own observations, so far at least as to cast away the idea of the progressive motion of light, which Roemer picked up and adopted.

Cassini only suspected the motion of light, from observing that the immersions of Jupiter's first satellite took place sooner and sooner in respect to the computed time, as they are observed from the conjunction of Jupiter to his opposition; and that the emersions took place later and later, as they are observed from Jupiter's opposition to his conjunction.

Now good philosophy would dictate, as it did to Cassini, that it would not be proper to ingraft a hypothesis or theory into the code of philosophy, upon so slight or doubtful evidence as the small apparent difference between the observed and computed time of a revolution of Jupiter's first satellite, lest we adopt false premises, and deduce wrong conclusions, which will only serve to bewilder and perplex us, in accounting for phenomena, and in making those necessary and rational deductions and conclu- 
sions, so necessary in support of a true theory; while hasty and absurd conclusions or deductions often serve only to lead us into a thousand empyric adaptations and adjustments, in making out and establishing our theories, from having in the outset overlooked some important point in the logical analogies of nature, which eventually lead on to false deductions.

From the foregoing, and from many other causes and difficulties that have existed and still exist, in the forming of correct tables of the eclipses of Jupiter's satellites, it may well be doubted, (as I have already remarked,) whether the theory of a progressive motion of light, would have been adopted as an established truth in philosophy, from a renewal by Roemer of the momentary and cast off hypothesis of Cassini, had not Dr. Bradley soon after seized upon the hypothesis, to aid him in accounting for an apparent motion of the fixed stars; which was supposed not only to confirm the theory of Roemer, but to a degree of exactness and certainty that could never be hoped for or expected from Jupiter's satellites.

But fully consenting to the very careful and critical observations of Dr. Bradley, upon the fixed stars, and their apparent motions, as by him observed, should his theoretic conclusions deduced therefrom prove unphilosophical and absurd, on further investigation, it may yet be hoped that we shall again observe the sun in its true place; that we shall see it where it is, and not where it is not; and that astronomers will be relieved from a thousand empyric equations, adopted for the purpose of making error as beautiful as truth.

I now propose to examine the evidence furnished by Dr. Bradley, in support of the theory of the progressive motion of light in time, as deduced from his observations. And in doing so, I shall avail myself of the common terms and phrases usually applied to light, considered both as a substance, and progressive in time, as the rays of light, the particles of light, the motion of light, the emission of light, \&c. ; believing, however, that a better vocabulary of terms, as applied to light, might be adopted, in which specific terms would not convey to the mind a whole body of false philosophy.

I will not say that I am intending to prove a negative, - to prove that light is not progressive in time, or that time is not necessary to the perception of a distant luminous body; but rather, that sufficient facts or data have not as yet been ascertained, to prove that light is progressive in time. And should those views prove correct, they will further serve to show the propriety of giving to a hypothesis a logical investigation previous to applying to it mathematical and geometric adaptation, 
and supposed demonstration, lest paradoxes, dilemmas and absurdities, intrude themselves, to the overturning of the theory, if it be not true in fact.

Dr. Bradley's observations upon the fixed stars, from whence he derived his proof as to the progressive motion of light, were made as early as the year 1728, an account of which he communicated to Dr. Halley, in a letter containing the results of his observations, and the final conclusions he had drawn from the same. And that Dr. Bradley may be heard in respect to his observations upon the fixed stars, together with his deductions drawn therefrom, I will here give such parts of his letter as directly refer to or serve to show his manner of observation, together with his conjectures and conclusions; not quoting those portions of his letter which only serve to show the method of fixing up his instrument, \&c. He says :

"Mr. Molyneux's apparatus (a zenith sutor) was completed and fitted for observing, about the end of November, 1725, and on the third day of December following, the bright star in the head of Draco, (marked $\gamma$ by Bayer, called Draconis,) was for the first time observed, as it passed near the zenith, and its situation carefully taken with the instrument. The like observations were made on the 5th, 11th, and 12th of the same month; and there appearing no material difference in the place of the star, a farther repetition of them at this season of the year, seemed needless, it being a part of the year wherein no sensible alteration of parallax in this star could soon be expected. It was chiefly, therefore, curiosity that tempted me, (being then at Kew, where the instrument was fixed,) to prepare for observing the star on December 17th, when, having adjusted the instrument as usual, I perceived that it passed a little more southwardly this day, than when it was observed before. Not suspecting any other cause of this appearance, we at first concluded that it was owing to the uncertainty of the observations, and that either this or the foregoing was not so exact as we had before supposed; for which reason, we purposed to repeat the observation again, in order to determine from whence this difference proceeded; and upon doing it on December 20th, I found that the star passed still more southwardly than in the former observations. This sensible alteration the more surprised us, in that it was the contrary way from what it would have been, had it proceeded from an annual parallax of the star; but being now pretty well satisfied that it could not be entirely owing to the want of exactness. in the observations, and having no notion of anything else that could cause such an apparent motion as this in the star, we began to think that some change in the materials, \&c., of the 
instrument itself might have occasioned it. Under these apprehensions we remained some time, but being at length fully convinced, by several trials, of the great exactness of the instrument, and finding by the gradual increase of the star's distance from the pole, that there must be some regular cause that produced it, we took care to examine nicely, at the time of each observation, how much it was; and about the beginning of March, 1726, the star was found to be $20^{\prime \prime}$ more southwardly than at the time of the first observation. It now indeed seemed to have arrived at its utmost limit southward, because in several trials made about this time, no sensible difference was observed in its situation.",

By the middle of April, it appeared to be returning back again towards the north; and about the beginning of June it passed at the same distance from the zenith as it had done in December, when it was first observed. "From the quick alteration of this star's declination about this time, (it increasing a second in three days,) it was concluded that it would now proceed northward, as it before had gone southward of its present situation; and it happened as was conjectured, for the star continued to move northward till September following, when it again became stationary, being then near $20^{\prime \prime}$ more northwardly than in June, and no less than 39" more northwardly than it was in March. From September the star returned towards the south, till it arrived, in December, to the same situation it was in at that time twelve months, allowing for the difference of declination on account of the precession of the equinox.

"This was a sufficient proof that the instrument had not been the cause of this apparent motion of the star, and to find one adequate to such an effect seemed a difficulty. A nutation of the earth's axis was one of the first things that offered itself upon this occasion; but it was soon found to be insufficient; for though it might have accounted for the change of declination in $\gamma$ Draconis, yet it would not at the same time agree with the phenomena in other stars, particularly in a small one almost opposite in right ascension to $\gamma$ Draconis, at about the same distance from the north pole of the equator; for though this star seemed to move the same way as a nutation of the earth's axis would have made it, yet it changed its declination but about half as much as $\gamma$ Draconis in the same time, (as appeared by comparing the observations of both made upon the same days at different seasons of the year;) this plainly proved that the apparent motion of the stars was not occasioned by a real nutation, since if that had been the case, the alteration in both stars would have been nearly equal. 
"The great regularity of the observations left no room to doubt but that there was some regular cause that produced this unexpected motion, which did not depend on the uncertainty or variety of the seasons of the year. Upon comparing the observations with each other, it was discovered that in both the fore-mentioned stars, the apparent difference of declination from the maxima was always nearly proportional to the versed sine of the sun's distance from the equinoctial points. This was an inducement to think that the cause, whatever it was, had some relation to the sun's situation with respect to those points. But not being able to frame any hypothesis at that time sufficient to solve all the phenomena, and being very desirous to search a little farther into this matter, I began to think of erecting an instrument for myself at Wanstead," \&c.

After giving the preparation of his instrument, he says, "my instrument being fixed, I immediately began to observe such stars as I judged most proper to give me light into the cause of the motion already mentioned. There was variety enough of small ones, and not less than twelve that I could observe through all the seasons of the year, they being bright enough to be seen in the daytime when nearest the sun. "I had not been long observing before I perceived that the notion we had before entertained of the stars' being farthest north and south when the sun was about the equinoxes, was only true of those that were near the solstitial coloure; and after I had continued my observations a few months, I discovered what $I$ then apprehended to be a general law, observed by all the stars, viz., that each of them became stationary or was farthest north or south when they passed over my zenith at six o'clock either in the morning or evening. I perceived likewise that whatever situation the stars were in with respect to the cardinal points of the ecliptic, the apparent motion of every one tended the same way when they passed my instrument about the same hour of the day or night; for they all moved southward while they passed in the day, and northward in the night; so that each was farthest north when it came about six o'clock in the evening, and farthest south when it came about six in the morning.

c. "Though I have since discovered that the maxima in most of these stars do not happen exactly when they come to my instrument at those hours, yet not being able at that time to prove the contrary, and supposing that they did, I endeavored to find out what proportion the greatest alterations of declination in different 'stars bore to each "other; it being very evident that they did not all change their declinations equally.

"I have before taken notice that it appeared from Mr. Moly- 
neux's observations that $\gamma$ Draconis altered its declination about twice as much as the fore-mentioned small star almost opposite to it ; but examining the matter more particularly, I found that the greatest alteration of declination in these stars was as the sine of the latitude of each respectively. This made me suspect that there might be the like proportion between the maxima of other stars; but finding the observations of some of them would not perfectly correspond with such an hypothesis, and not knowing whether the small difference I met with might not be owing to the uncertainty and error of the observations, I deferred the farther examination into the truth of this hypothesis till I should be furnished with a series of observations made in all parts of the year; which might enable me not only to determine what errors the observations are liable to, or how far they may safely be depended upon; but to judge whether there had been any sensible change in the parts of the instrument itself.

"Upon these considerations I laid aside all thoughts at that time about the cause of the fore-mentioned phenomena, hoping that I should the easier discover it when I was better provided with proper means to determine more precisely what they were.

"When the year was completed I began to examine and compare my observations, and having pretty well satisfied myself as to the general laws of the phenomena, I then endeavored to find out the cause of them. I was already convinced that the apparent motion of the stars was not owing to a nutation of the earth's axis. The next thing that offered itself, was an alteration in the direction of the plumb line with which the instrument was constantly rectified; but this upon trial proved insufficient. Then $I$ considered what refraction might do, but here also nothing satisfactory occurred. At last I conjectured that all the phenomena hitherto mentioned, proceeded from the progressive motion of light and the earth's annual motion in its orbit. For I perceived if light was propagated in time, the apparent place of a fixed object would not be the same when the eye is at rest, as when it is moving in any other direction than that of the line passing through the eye and object; and that when the eye is moving in different directions, the apparent place of the object would be different.".

This is "Dr. Bradley's account of this very important discovery," and many pages are filled with supposed geometrical and mathematical explanations or demonstrations of the truth of his hypothesis, and its adaptation to the equation of light, \&c., to which explanations and adaptations I must refer all who may be curious to know the vast amount of labor and investigation that has been bestowed upon this hypothesis. 
I have thought proper to bring forward Dr. Bradley's own account of his observations, and his theory or hypothesis deduced therefrom, that they may be readily compared with my attempted refutation of his theory, and with that which I may suggest in its stead.

We find also in the works on astronomy this further reference to Dr. Bradley's observations and deductions upon the same subject, viz.

Dr. Bradley further observes that in seventy observations made in a year on $\gamma$ Draconis, there was but one (and that is noted very dubious on account of clouds) which differed more than $2^{\prime \prime}$ from the theory, and that did not differ $3^{\prime \prime}$. And in about fifty observations made in a year on $\pi$ Ursa majoris, he did not find a difference of $2^{\prime \prime}$, except in one marked doubtful on account of the undulation of the air, \&c. and that did not differ $3^{\prime \prime}$. And this agreement between the theory and observation leaves no room to doubt but that the cause is rightly assigned. And, if this be the case, the annual parallax of the fixed stars must be extremely small. "I believe," says the Dr. "that I may venture to say that in either of the above mentioned stars, it does not amount to $2^{\prime \prime}$. I believe if it were $1^{\prime \prime}$, I should have perceived it in the great number of observations that I made, especially on $\gamma$ Draconis ; which agreeing with the theory (without allowing anything for parallax) nearly as well in conjunction with, as in opposition to this star, it seems very probable that the parallax is not so great as one single second; and consequently that it is above four hundred thousand times further from us than the sun." The observations of Mr. Flamstead of the different distances of the pole star from the pole at different times of the year, and which was looked upon as a proof of its annual parallax, was undoubtedly owing to this cause. For he concluded that the star was $35^{\prime \prime}, 40^{\prime \prime}$ or $45^{\prime \prime}$ nearer the pole in December than in May or July; and according to this hypothesis, it ought to appear $40^{\prime \prime}$ nearer in December than in June. This agreement is greater than could have been expected from observations made with his instrument."

Hence Dr. Bradley deduced the following conclusions : 1 . "That the light of all the fixed stars arrives at the earth with equal velocities; for the major axis of the ellipse is the same in all the stars; that is $40^{\prime \prime}$, according to his last determination. 2. That unless their distances from us are all equal, which is very improbable, their lights are propagated uniformly to all distances from them. 3. That light moves from the sun to the earth in $8^{\prime} 7^{\prime \prime} .5$, and its velocity is to the velocity of the earth in its orbit as $10314: 1$. 4 . That the time thus determined can scarcely err 
from the truth by above $5^{\prime \prime}$ or $10^{\prime \prime}$ at most, which is such a degree of exactness as can never be expected from the eclipses of Jupiter's satellites. 5. That as this velocity of star light comes out about a mean of the several velocities found from the eclipses of Jupiter's satellites, we may reasonably conclude that the velocity of these reflected lights are equal to the velocity of direct light. 6. And as it is highly probable that the velocity of the sun's emitted light is equal to that of star light, it follows that its velocity is not altered by reflection into the same medium."

I have thought proper to insert the foregoing reference to Dr. Bradley's observations and theory, as furnishing (after most ample applications to practical utility as is conceived) a string of corollaries extending to such things as were conceived to fall within analogies.

Dr. Bradley in rendering a hypothesis to the phenomena which were the result of his observations, has made this assumption. "He perceived, that if light was propagated in time, the apparent place of a fixed object would not be the same when the eye is at rest, as when it is moving in any other direction than that of the line passing through the eye and object; and that when the eye is moving in different directions, the apparent place of the object would be different."

Now it appears to me this is begging the question; for notwithstanding the Dr. has carefully inserted the word if as a salvo, yet the world have assumed the premises as being true, and have reasoned more critically on the subject than I am able to comprehend as being either sound or true, notwithstanding their explanation of the laws of motion upon the principles of a parallelogram or resolution of forces, and their finally assumed proportions between the velocity of light, and that of the earth in its orbit, as sometimes attempted to be explained by conceiving a very long telescope or tube of sufficient calibre only to permit a single ray or particle of light to flow down in the tube; and so adjusted in respect to the motion of light and the motion of the earth in its orbit, that the ray or particle of light would flow freely down the tube or telescope; and that the eye placed at the lower end of the tube would perceive the ray or particle of light giving the appearance of the star or object from which the light is emitted, in the line of the tube; notwithstanding the tube or telescope would not lie in the line from the eye to the object; but so situated as to catch the rays or particles of light in advance of such direct line from the eye to the object. But this mode of reasoning appears to me to overlook some of the plainest: and best established principles and facts in the science of optics; for although I have long considered the 
adopted theories on light to be vague and unphilosophical, nevertheless, the theory of optics, so far as regards the effects or operations of light, is in many respects as beautifully and satisfactorily defined and explained as that of any other branch of science or philosophy; among its laws are those which teach us that the eye discerns an object in the precise direction in which the eye receives or obtains the light, whether the light which presents the object to the eye be direct from the object to the eye, or whether it may have been reflected or refracted; but when the light that presents the object to the eye has been refracted, for the very reason that the eye discerns the object in the exact direction in which it receives the light which presents the object, the apparent place of the object will not be its real.

And as well might we expect to see the sun at noon day by looking in any other direction than towards it, (inasmuch as its rays are falling thick all around,) as to perceive the star by looking at those rays or particles of light (as they are called) which are yet in advance of a direct line from the eye to the star; and the idea is preposterous that we can see a ray or particle of light flowing down the telescope or tube, not directed towards the eye, but to a place in advance of the eye, merely because the eye and ray or particle will presently meet in the angle of the requisite parallelogram. And it is not pretended even by way of explanation of Dr. Bradley's theory, that the line of direction of the telescope or tube is in that from the eye to the star, but as in the case of a composition of forces, an angle is formed by the line of the eye and object with the line of the motion of the eye, equal to what is conceived to denote the proportional velocities of light, and of the eye.

And to show how the progressive motion of light may affect the place of the star seen by the naked eye, they have in reality, only made the eye a shorter tube, or telescope; and hence it is said that, "if a ray of light fall upon the eye in motion, its relative motions in respect to the eye, will be the same as if you were to impress equal motions in the same direction, upon each at the instant of contact; for equal motions in the same direction, impressed upon two bodies, will not affect their relative motions, and therefore, the effect of one upon the other, will not be altered." And another parallelogram of forces, or of motion, is presented by way of diagram, to exemplify the truth of the position.

But, if philosophers had abided by all experience, and by what all sound philosophy has taught, as to the eternal fitness of things, in adapting the mechanism of the eye to our best convenience, so that when it discerns an object, the crystalline lens, and other parts of the eye, are so constructed, as to present the 
object in a direct line from the eye, unless the light has been reflected, or refracted; and which, of all the laws of optics, is most dear to us, as presenting the object so that we may see it where it is, rather than where it is not; - they would, perhaps, not have attempted to filch enough from the well known and best established laws of optics, to make a philosophic truth of Dr. Bradley's conjecture and final perception, that if light was propagated in time, it would solve all the phenomena arising from his observations, by assuming, for the purpose of explanation, that such is the fact; and by those subtleties which may sometimes "make the worse appear the better reason," impose the hypothesis upon a credulous world, as being demonstrated or proved; and therefore requiring no farther investigation. But I think, however specially, or critically, they may have pleaded in the case, that the world, on a re-examination of the case, will adjudge that they take nothing by their plea.

One result of the Doctor's theory would be, that the nearer a planet is to the sun, the greater will be the difference between the true and apparent place of the sun, as observed from such planet. This, however, would be no direct or conclusive argument against his theory or hypothesis.

I will now attempt to show some results, that must necessarily follow or flow from Dr. Bradley's hypothesis, when tested by an atternpt at demonstration. But, before suggesting a diagram, $\mathbf{I}$ will quote from explanations of Dr. Bradley's theory, in order to justify myself in the form or construction of the diagram which I have chosen to adopt.

1. It is assented to by the authorities, and will be readily assented to by all, that for all purposes of explanation or of demonstration, so far as the principle of the aberration of light may be concerned, a circular orbit, with the sun in the centre, is as proper as an eccentric or elliptical orbit; the only difference consisting in this : that in a circular orbit, the motion of the earth, and of course, of the eye of the observer, would be uniform; while in an eccentric orbit, it would be something greater at the perihelion than at the aphelion; but even our earth's orbit is so nearly a centric orbit, as to permit astronomers to consider it as such, so far as the aberration of light is concerned, as well as in many other calculations.

2. It is said that, "Whilst the earth makes one revolution in its orbit, the curve, parallel to the ecliptic, described by the apparent place of a fixed star, is a circle,"-to which all will assent.

3. It is said also, that " in the plane of the ecliptic, the sine of the star's latitude being 0 , the orbit becomes a straight line," \&c. 
That is, to an observer, placed in the plane of the ecliptic, at a great distance from the line of the earth's orbit, if such line were a visible line, it would also appear to be a straight line.

4. It is also said "to find the aberration in latitude or longitude, let A B C D, be the earth's orbit, supposed to be a circle, with the sun in the centre," \&c.

Hence, in the diagram suggested, I shall conceive the star to be observed, for the purpose of getting its apparent motion, or what is termed its aberration, to be in the plane of the ecliptic, but at such an infinite or indefinite distance from the earth, in any part of its orbit, as to have no sensible parallax, or actual motion or change of place, even by the nicest observations; in which case, I think, the diagram will be assented to, as wholly proper for investigating the apparent motion of such star, caused by the revolution of the earth in its orbit.

And to avoid any inconvenience or perplexity in considering the different hours of the day, when the star observed comes to the meridian where the observer is situated, occasioned by the diurnal revolution of the earth on its axis, I have conceived only the eye of the observer, to revolve around the sun in the orbit of the earth, with the same motion with which the earth revolves; in which case, the eye of the observer would at all times see the star; and that in such case, the only apparent motion of such star, would be that of a backward and forward motion, as in a straight line, in the plane of the ecliptic, to an amount of $40^{\prime \prime}$ in the course of an annual revolution of the eye of the observer.

I will here, again recapitulate some of the phenomena arising from Dr. Bradley's observations, which appear most essential to be considered, with a view to a proper solution.

1. The apparent motion of the star, is always in the same direction in which the earth would appear to be moving, to an observer situated at such star.

2. In March, the star $\gamma$ Draconis, appeared stationary for many days, at its utmost southern limit.

3. In June, the star had passed $20^{\prime \prime}$ north of its place in March, and was continuing northward, having then its most rapid motion.

4. It continued to pass northward until September, when it was $40^{\prime \prime}$ north of its place in March, and where it again became stationary for some days.

5. In September, it commenced returning to the south, and in December, it was $20^{\prime \prime}$ farther south than it was in September, having again in December, its most rapid motion.

6. In March following, it had again arrived at its most southern limit, and after remaining stationary for a time, again commenced its return to the north. 
Let us now conceive a diagram of the orbit of the earth, with its requisite appendages, with the star to be observed, situated at an indefinitely great distance, but in the plane of the orbit.

Conceive a straight line (called the zenith line) to be drawn from the fixed star to be observed, through the centre of the orbit, intersecting the line of the orbit in two points; the point of intersection next the star being denoted by $\mathrm{A}$, and signifying June, or the summer solstice; and the opposite point of intersection by $\mathrm{C}$, and signifying December, or the winter solstice.

Conceive another line drawn through the centre of the orbit, at right angles to the central line, intersecting the line of the orbit in two points ; one being denoted by $\mathbf{B}$, and signifying September, or the autumnal equinox; and the other denoted by $\mathrm{D}$, signifying the vernal equinox ; which said line so intersecting the orbit, may be called the equinoctial line.

The points of intersection of the orbit by the central line, may be called the zenith points, and the others the equinoctial points.

Conceive tangent lines to the orbit to be drawn from the equinoctial points, extending (parallel to each other) to the fixed star to be observed; for it must be understood, that notwithstanding the great diameter of the earth's orbit, it will not form the base to a scnsible triangle, the vertex of which is in a fixed star; and hence those tangent lines will be parallel to each other to an exactness beyond detection. That tangent proceeding from B, being called the $\mathrm{B}$ tangent, and that proceeding from $\mathrm{C}$, the $\mathrm{C}$ tangent. Then a body revolving in the orbit would, to an observer at such fixed star, appear to pass backward and forward upon the equinoctial line.

Now conceive the eye of the observer revolving in the orbit in the direction D A B C, and to have arrived at D, or the vernal equinox, when the star will be stationary, and $20^{\prime \prime}$ further south than it will be when the eye arrives at A, and $40^{\prime \prime}$ further south than when the eye arrives at B. And, according to Dr. Bradley's theory, the reason why the star appears stationary at $\mathrm{D}$ or at $\mathrm{B}$, is because the eye is then passing in the line of the eye and object, or star. Hence at D the star must appear $20^{\prime \prime}$ south of the tangent line at $\mathrm{D}$, or $20^{\prime \prime}$ out of its true place for days, and at the time and place when the eye is passing in a line towards the star, and not transverse to the line of the eye and object. When the eye arrives at $\mathrm{A}$ its motion is directly transverse perpendicular to the line of the eye and star; and the star then appearing $20^{\prime \prime}$ further north than when the eye was at $\mathrm{D}$, and $20^{\prime \prime}$ further south than it will appear when the eye arrives at B; and hence a mean between the two extremes, it will then at $\mathbf{A}^{\prime}$ appear directly in the zenith, or zenith line; and this at the time 
when its apparent motion northward is most rapid; and when, according to Dr. Bradley's theory, the aberration of the star should be greatest.

When the eye arrives at $\mathrm{B}$ it views the star $20^{\prime \prime}$ to the north of the tangent line drawn from B; and there the star again becomes stationary for days, because the motion of the eye is in the line of the eye and star, or object viewed. As the eye proceeds from B to C, the star returns southward; and when the eye arrives at $\mathrm{C}$, the star will again appear in its true place, in the zenith line, although its apparent motion will then be most rapid towards the south, and the motion of the eye will then be transverse to the line of the eye and star; and the motion of the star will continue southward till the eye again arrives at $\mathrm{D}$, when it will again become stationary at $20^{\prime \prime}$ out of its true place ; when it will commence going north again.

Now it would seem as though no further explanation need be made to convince the most casual observer of the entire absurdity of Dr. Bradley's theory. For I will ask whether any one can reconcile the Doctor's theory with the fact, that when the motion of the eye transverse to the line of the eye and star is greatest, the star should be in its true place; and when the requisite motion of the eye for producing an aberration is nothing, because it is in a line of the eye and object, the aberration of the star should not only be the greatest, but continue so for days, namely, while the star should appear stationary?

But again, - the amount of aberration at $\mathrm{D}$ or at $\mathrm{B}$ is only 8 1. 4 th minutes in time. Hence ${ }_{x}$ if the eye were to remain still, or were to be passing in the line of the eye and star for that length of time, the star must necessarily come to its true place, even upon Dr. B.'s theory. But at D or B the eye has no requisite motion for producing any aberration of the star for days, and yet the star stands out south at D and north at B, for all this time.

But when the eye comes to $A$ or to $C$, the star appears in its true place, being a mean between its north and south declination, notwithstanding the motion of the eye is then transverse to the line of the eye and star; and as the motion of the star is then the greatest, then according to the Doctor's theory, that if light be progressive, and this be the cause of the apparent motion of the star, its greatest aberration should be when the eye is at $\mathrm{A}$ or $\mathrm{C}$; but it is directly the reverse, and goes as far as a theory can to prove that light is not progressive in time.

This would seem to indicate that were the motion of the eye to cease in any part of the orbit, the star would continue to remain at the place where it appeared when the motion of the eye ceased; and so doubtless would be the fact; for it is too obvious that the aberration of light, upon Dr. Bradley's theory, is not the 
cause of the phenomena, which doubtless depends on a much more natural, simple and beneficial cause, which I may hereafter venture to suggest.

Perhaps the only rational way of accounting for the error which those have fallen into who have undertaken to explain the Doctor's theory, is, by supposing that they erred as to what places or points of the orbit the star would be seen in its true place; and we must conclude that they made the true place where the star would appear when stationary; for three days were allowed for it to come to its true place, when at most but a few minutes would be required; not considering that at those points where the star appears stationary, lines drawn to the two apparent places of the star from those points would form an angle of $40^{\prime \prime}$; and that at $\mathrm{A}$ and $\mathrm{C}$ the star from both points would appear in the same point in the heavens, and that, too, in the mean and the true place.

But it will be perceived that the star appears in its true place only when the motion of the eye is in a direct transverse direction to the line of the eye and star; and that in no other parts of the orbit will the eye perceive the star in its true place, going upon the true theory, whatever that may be.

And hence I think it clear that if the phenomena observed by Dr. Bradley in respect to the apparent motion of the fixed stars, prove anything, they prove directly the opposite of what has generally been supposed; and I think it conclusively shown that the motion of the eye, whether it be much or little, has nothing to do in accounting for the phenomena observed by Dr. Bradley; and if any have supposed that the star appeared in its true place at $B$ and at $D$, let them attempt to extricate themselves from such a dilemma.

A nutation of the poles of the earth is sometimes defined to be " a kind of tremulous motion of the axis of the earth, by which in its annual revolution, it is twice inclined to the ecliptic, and as often returns to its former position"- - and so far as the actual poles of the earth partake of a circular motion, or revolution about the mean poles of the earth, such revolution is found to be in the order of the signs.

Dr. Bradley found in his critical observations that the stars which he observed, apparently had their most rapid motion near the winter and summer solstices, and their least apparent motion near the vernal and autumnal equinoxes; and that a star situated near the solstitial colures, would have a greater apparent motion either north or south, than those situated near the equinoctial colures. The Dr. also found, that it was not a general law of the apparent motion of the stars that they would be 
farthest north or south when the sun was about the equinoxes; but that such only held good in respect to those stars that were near the solstitial colure; nevertheless, any star would become apparently stationary (being farthest either north or south) when it passed over his zenith about six o'clock in the morning, or six o'clock in the evening; being farthest south when it passed at six o'clock in the morning; and farthest north when -it passed at six o'clock in the evening; the apparent motion of any star tending the same way when it passed his instrument about the same hour of the day or night, with the exception of some small variation.

Now notwithstanding all this evidence, the Dr. says that on examining and comparing his evidence, and having pretty well satisfied himself as to the general laws of the phenomena, he then endeavored to find out the cause of them; that he was already convinced that the apparent motion of the stars was not owing to a nutation of the earth's axis. But I am certainly at a loss to know from what cause his conviction arose - inasmuch as all the phenomena detailed or set forth would seem to refer the nutation of the earth's axis and the apparent motion of the stars to the same cause.

But the Dr. says, at last he conjectured the cause \&c., which conjecture has ever since been received and acted upon as the true hypothesis. But it would seem as though most philos. ophers with all this evidence before them, would have conjectured that one and the same cause produced the nutation of the earth's axis, the precession of the equinoxes, and the apparent motion of the fixed stars; not however, unless they conceived the solar system to be governed by some immutable law of order, in lieu of being governed by fortuity, or the Newtonian theory of universal gravity, which would indeed very illy account for those phenomena.

But if we conceive the solar system to be governed by laws of order, those phenomena, viz. the nutation of the poles, the precession of the equinoxes, and the apparent motion of the stars observed by Dr. Bradley, would seem to be the direct and natural results of such law, and some of the most happy elucidations of the law. In such case there would be no lunar nutation, but the whole would be solar nutation; nor would it be said as is now said, that physical astronomy has made known a solar nutation, but that it is not sufficient to be detected by observation; but if a law of order for the solar system were known and acknowledged, it would dictate to us that such phenomena must necessarily occur; hence on observing their occurrence there would be no difficulty in assigning the true cause. 


\section{A P P E N D I X.}

I have thought proper, in a short appendix, to add something further in reference to the quadrature and its general application ; as also some few additional remarks in reference to the law of gravity.

As heretofore, the $p$ whose $\mathrm{M} a$ and $\mathrm{C}$ are conceived to be adverse, is denoted by figure 1 at the right of its respective denotations; and the $p$ whose $\mathrm{B}$ and $\mathrm{C}$ are conceived to be adverse, and whose $\mathrm{M} a$ and $\mathrm{D}$ coincide in 2 is denoted by figure 2 at the right of its respective denotations.

$\mathrm{M} a$ and $\mathrm{B}$ of the $p$ of twice the sides of the $p 2$ occupy the identical places that $\mathrm{C}$ and $\mathrm{D}$ of the $p$ do whose $\mathrm{A}$ and $\mathrm{B}$ coincide in 2 .

$\mathrm{C}$ and $\mathrm{B}$ of the $p 2$ occupy the identical places that $\mathrm{B}$ and $\mathrm{D}$ do of the $p$ whose $\mathrm{C}$ is in 2 .

$\mathrm{M} a$ and $\mathrm{C}$ of the $p 1$ occupy the identical places that $\mathrm{A}$ and $\mathrm{M} a$ do of the $p$ whose $\mathrm{C}$ is in 2 .

The adverse of $\mathrm{A}$ of any given $p$ is a mean between 2 and $\mathrm{E}$ of a like $p$, and is consequently $=m 2$ when the area is $=\mathrm{E}$.

The adverse of $\mathrm{M} a$ of any given $p$ is a mean between 2 and $\mathbf{G}$ of a like $p$, and is consequently $=m 2$ when the area is $=\mathrm{G}$.

The adverse of a mean between $\mathrm{A}$ and $\mathrm{M} a$, narnely, the adverse of $\mathrm{C}$, is a mean between 2 and $\mathrm{E}$ of twice the sides, and is consequently $=m 2$ when the area is $=\mathrm{E}$ of twice the sides.

The mean place of $\mathrm{M} a$ is a mean between $\mathrm{M} a$ and $\mathrm{C}$ of a $p$ of half the sides.

The mean place of $\mathrm{D}$ is a mean between $\mathrm{B}$ of the same $p$, and $\mathrm{D}$ of a $p$ of half the sides.

The square of the mean place of $\mathrm{M} a$ is a mean between the square of the true place, and the square of $\mathrm{E}$ of a $p$ of twice the sides. 
The square of the mean place of $\mathrm{D}$ or of $\mathrm{C}$, is a mean between the square of the true place of $D$, and the square of the true place of $\mathrm{C}$; and the square of the mean place of $\mathrm{A}$ is a mean between the square of the true place of $\mathrm{D}$ of half the sides, and the square of the true place of $\mathrm{C}$ of half the sides.

The product of $\mathrm{M} a 4$ by $\mathrm{C}$, is 2 above the square of $\mathrm{M} a 8,-$ the product of $\mathrm{M} a 8$ by C 8 , is $\frac{1}{2}$ above the square of $\mathrm{M} a 16$, the product of $\mathrm{M} a 16$ by $\mathrm{C} 16$, is $\frac{1}{2}$ above the square of $\mathrm{M} a 32,-$ and so on, until the product of $\mathrm{M} a \mathbf{1}$ by $\mathrm{C} 1$, is the square of M $a$ 2. And if we attempt to continue a like progression, we shall find a uniformity which has not previously existed; for the product of $\mathrm{M} a 2$ by $\mathrm{C} 2$, is = the square of $\mathrm{M} a$ of twice the sides, and such will continue to be the case, however far we attempt to continue the progression. Hence, the product of $\mathrm{M} a$ by $\mathrm{C}$, can never be below the square of $\mathrm{M} a$ of a $p$ of twice the sides; and hence, from the $p 1$ onward, our progression is futile and void.

The product of $\mathrm{M} a$ by $\mathrm{D}$, is always = the product of $\mathrm{B}$ by $\mathrm{C}$, or of $\mathrm{B}$ of the given $p$ by $\mathrm{A}$ of a $p$ of twice the sides; and as in the circle, $\mathbf{M} a$ actually coincides with $\mathrm{C}$, hence, our conception necessarily is, that in the circle, $M a$ coincides with $A$ of twice the sides.

When $\mathrm{D}$ is conceived to be 1 below $\mathrm{M} a$, such $\mathrm{D}$ is the adverse of the popular D, or B of the circle; and such $\mathrm{M} a$ is the adverse of the popular. $\mathrm{M} a$ or $\mathrm{C}$ of the circle; and such $\mathrm{M} a$ is then a mean between $\mathrm{D}$ and $\mathrm{B}$ of the same $p$, and is consequently $=\mathrm{B}$ of a $p$ of twice the sides.

$\mathrm{B} 2$ is conceived to be the farther of two means from B 1 , to 2 - and the popular $\mathbf{B}$ of the circle, is the farther of two means from $B 2$ to 2 .

2 is the farther of two means from $A$ to $B$, hence, when $A$ and $B$ coincide in ${ }^{2}$, such point is as well the farther of two means from $A$ to $B$ of twice the sides, as from $A$ to $B$ of half the sides.

If we conceive a $p$ of twice the sides of that whose A and B coincide in $\underset{2}{2}$, we necessarily conceive $M a$ of such $p$ to be $\frac{2}{2}$ below 2 , or in the place of $\mathrm{C} 1$, or of $\mathrm{A} 2$; and also, that $\mathrm{A}$ of such $p$ is $\frac{2}{2}$ above $\stackrel{2}{2}$, or in the place of $\mathrm{M} a 1$; and that $\mathrm{C}$ of such $p$, is in 2 , and $\mathrm{B}$ of such $p$, will be conceived to be 1 below $\underbrace{2}_{2}$ and $\mathrm{D} 1$ above 2 . If in the $p 1$ we conceive 1 to poss 'ss the same value as in the $p 8$ and still conceive $\mathrm{B}$ to continue 1 above $\mathrm{M} a$ and $\mathrm{D}$ to continue to be 1 above $\mathrm{C}$, and attempt to continue the progression beyond the $p 1$ and until $\mathrm{B}$ and $\mathrm{D}$ coincide 1 above $M a$ and $C$, we necessarily place $B$ and $D$ final $\frac{1}{3}$ above ${ }^{2}$, and $\mathrm{M} a$ and $\mathrm{C}$ final $\frac{2}{3}$ below 2 : But, as before remarked, from the $p 1$ onward, we do not displace $\mathrm{M} a$ from its constant mean position between $\mathrm{M} a$ and $\mathrm{C}$ of $p$ of half the sides. 
So also, if we conceive the progression to be continued after $\mathrm{A}$ and $\mathrm{B}$ coincide in $\underset{2}{2}$, we shall arrive at the same conceived result, namely, shall place $\mathrm{B}$ and $\mathrm{D}$ final $\frac{1}{3}$ above $\stackrel{2}{2}$, and $\mathrm{M} a$ and $\mathrm{C}$, final $\frac{2}{3}$ below $\underbrace{2}_{2}$; but it is manifest that such continued progression will be by an apparent retrograde movement of the denotations from that $p$ which is conceived to have twice the sides of that whose $\mathrm{A}$ and $\mathrm{B}$ are in 2 . That is, when $\mathrm{C}$ is in $\stackrel{2}{2}$, we conceive $\mathrm{M} a$ to be 1 below $\mathrm{M} a$ when $\mathrm{A}$ is in $\underset{2}{2}$; and if $\mathrm{B}$ continues to be $\frac{1}{\text { above }} \mathrm{M} a$, and if $\mathrm{B}$ of the circle is 1 above $\mathrm{M} a$ of the circle (if such $\frac{1}{-}$ then has any value, whatever value it may then have,) the final $B$ and $D$ must be $\frac{1}{3}$ (of such value) above 2 , and $M a$ and $C$ final must be ${ }_{3}^{2}$ (of such value) below 2 ; but if in the circle there is no value to $\stackrel{1}{\rightarrow}$, then $\mathrm{M} a$ and $\mathrm{D}$ of the circle are in 2 .

In such case, $\mathrm{A}$ in ${ }^{2}$ must be conceived to be $\mathrm{A}$ of twice the sides of $\mathrm{M} a$ of the circle; and consequently, we must conceive $\mathrm{A}$ of half the sides of $\mathrm{A}$ in 2 , to be in reality the utmost $\mathrm{A}$, and it is manifest that we can only conceive such $A$ of half the sides to be $=\mathrm{M} a 1$. And the product of $\mathrm{M} a 1$ by $\mathrm{C} 1$ is the square of the true place of M $a 2$, and of the mean place of. M $a$ of four times the sides of the $p 1$, and the like may be conceived in respect to the circle. Hence, in $\underset{2}{2}$, the true and mean places of $\mathrm{M} a$ coincide, as they do in the $p 4$.

Hence $\mathrm{M} a$ in $\underset{2}{2}$, has risen to a mean between $\mathrm{M} a$ and $\mathrm{C}$ of a $p$ of half the sides; and the popular methods do not attempt anything farther. $\quad \mathrm{M} a \mathrm{~S}$ is 1 below a mean between $\mathrm{M} a$ and $\mathrm{C}$ of a $p$ of half the sides; hence, 1, which in the $p 8$ denotes a mean between $\mathrm{M} a$, and a mean between $\mathrm{M} a$ and $\mathrm{C}$ of a $p$ of half the sides, is equated to $\underline{0}$ in the $p 2$; and no one expects that $\mathrm{M} a$ will either rise above a mean between $\mathrm{M} a$ and $\mathrm{C}$ of a $p$ of half the sides, or that the law of progression will so far change, that $\mathrm{M} a$ will continue (while the progression continues) to be a mean between $\mathrm{M} a$ and $\mathrm{C}$ of a $p$ of half the sides.

$\mathrm{M} a$ then coincides with $\mathrm{M} a$ and $\mathrm{C}$ of half the sides, at the time and place when it becomes a mean between them, and then it necessarily coincides with $\mathrm{C}$ of the same $p$, thus making the four-fold chord of the periphery of the circle.

Thus, by the law of polygonal progression, M $a$ cannot be below 2 unless it be above a mean between $\mathrm{M} a$ and $\mathrm{C}$ of half the sides; and by any method, it is supposed that when $\mathrm{M} a$ becomes a mean between $\mathrm{M} a$ and $\mathrm{C}$ of half the sides, it coincides with them.

Suppose then, that the difference between $\mathrm{M} a 1$ and B 1 , or between $\mathrm{M} a 2$ and $\mathrm{B} 2$, to have even been increased in value from the $p \mathrm{~S}$, according to the popular increase, and then attempt to obtain $\mathrm{M} a$ of twice the sides of the $p 1$ in the popular man- 
ner,- that is, by dividing the product of $\mathrm{M} a 1$ by $\mathrm{C} 1$, by half the sum of $\mathrm{M} a \mathbf{1}$ and $\mathrm{C} 1$.

In such case, $\mathrm{M} a$ of a $p$ of twice the sides of the $p 1$ will be proportionally as far below 2 as an equal mean between $\mathbf{M} a 1$ and $\mathrm{C} 1$ is above 2 ; that is, if $\mathrm{M} a 1$ and $\mathrm{C} 1$ are not in 2 , by such process, $M a$ of twice the sides, will be below ${ }^{2}$, and consequently, must be above a mean between $\mathrm{M} a 1$ and $\mathrm{C} 1-$ which cannot be the case - neither can such $\mathrm{M} a$ be below ${ }_{2}^{2}$ for when $\mathrm{M} a$ and $\mathrm{C}$ are adverse, $\mathrm{M} a$ of twice the sides is necessarily in 2 . Thus, showing direct error, in the popular mode of conducting the progression.

$\mathrm{As} \mathrm{C}$ is a mean between $\mathrm{M} a$ of the same $p$ and $\mathrm{C}$ of half the sides, if after obtaining the popular $\mathrm{M} a 2$, we then attempt to obtain $\mathrm{C} 2$, we shall obtain it proportionally, half as far too low as the popular M $a 2$ will be; and it will be manifest to any one, that the error will continue to increase during the progression. But as $\mathrm{M} a$ cannot become a mean between $\mathrm{M} a$ and C of a $p$ of half the sides till it coincides with them; and as $\mathrm{M} a$ in $\underset{2}{2}$ is a mean between $\mathrm{M} a$ and $\mathrm{C}$ of a $p$ of half the sides, hence, $\mathrm{M} a$ in 2 coincides with $\mathrm{M} a$ and $\mathrm{C}$ of half the sides.

And as in the case of $\mathrm{M} a$ in $\stackrel{2}{2}$, in which $\mathrm{M} a$ has then risen to a mean between $M a$ and $C$ of a $p$ of half the sides, so D in 2 has fallen to a mean between $\mathrm{B}$ of the same $p$, and $\mathrm{D}$ of a $p$ of half the sides. Hence, in $\stackrel{2}{2}$, the relative situation of $\mathrm{D}$ will have altered in respect to a mean between $\mathrm{B}$ of the same $p$, and $\mathrm{D}$ of a $p$ of half the sides, from what it is in the $p 8$, proportionally half as much as the relative situation of $M a$ in 2 , in respect to a mean between $\mathrm{M} a$ and $\mathrm{C}$ of half the sides will have shifted from what it is in the $p 8$.

Hence, in 2 the mean places of both $\mathrm{M} a$ and $\mathrm{D}$ will be their true places; nor can $\mathrm{D}$ by rising above 2 , fall below a mean between $\mathrm{B}$ of the same $p$, and $\mathrm{D}$ of a $p$ of half the sides, any more than $\mathrm{M} a$ by falling below $\underset{2}{2}$, can rise above a mean between $\mathrm{M} a$ and $\mathrm{C}$ of a $p$ of half the sides. Consequently, in 2 the progression is ended in respect to $M a$ and $D$, and hence, in 2 $\mathrm{M} a$ and $\mathrm{D}$ become $\mathrm{M} a$ and $\mathrm{D}$ of the circle, which are the final quantities sought. And as the mean place of $\mathrm{D}$ is a mean between $\mathrm{D}$ and $\mathrm{C}$, hence, when the true and mean place of $\mathrm{D}$ coincide, $\mathrm{D}$ is then $=\mathrm{C}$, hence, when $\mathrm{D}$ is in $\underset{2}{2}, \mathrm{C}$ is in $\underset{2}{2}$, as also $M a$.

Such then, gives the true or actual ratio between the diameter and circumference of the circle, the proportions of which may be expressed in the powers and roots of definite numerical quantities; making the circle in its infinite use and application a pleasant, rather than irksome task.

I will here notice a few additional facts, by way of application. 
The fifth power of $\mathrm{M} a$ of the circle, is $d$ of the circle, whose circumference is 1 , and if such be an orbit; the period of the planet will be $=$ one sixteenth of the circumference.

If the circumference of an orbit be 4 , the period is one eighth of the circumference; thus, if the circumference be quadrupled, the ratio of the period to the circumference is reduced one half. So when the circumference is 16 , the square of the period is $=$ to the circumference; and this occurs when the diameter of the orbit is half the square of the circumference of the prime circle.

If the diameter of the inscribed circle of a given $p$, be $=$ to the square of the circumference of a like major $p$, the circumference of such polygon, will be the third power of the circumference of such major $p$; hence, if the diameter of the circle be 10.07936 (square of the circumference of the prime circle) the circumference of such circle will be 32 .

The area of a circle is always twice the square of $\widehat{2}$ below $m$; so if $m$ of the circle be $\widehat{2}$ above two thirds of 1 , (namely, when $m$ of the circle, is .839946) the area is .888, ad infinitum; which area is $=$ the area of the $p 6$, when $m$ of such $p 6$ is $=\mathrm{B} 6$; as .888 is the area of the circle whose $m$ is two thirds of $b a$ of the circle, so also, it is half the area of the $\mu 4$ whose $m$ is two thirds of $b a$ of the $p 4$, and is twice the area of the $p 4$ whose $m$ is two thirds of $d a$ of the $p 4$.

When the area of the circle is .888 , ad infinitum, $m$ of such circle is the cube root of two thirds of the area, and is the reciprocal of the cube root of the square of M $a 3$. So four thirds of M $a$ of the circle is $b$ of the circle whose area is .888, ad infinitum, and four thirds of $\mathrm{M} a$ of the $p 4$ is $b$ of the square whose area is 888 , ad infinitum.

Three times the square of $\mathrm{M} a 4$ is the reciprocal of one-third of $\mathrm{M} a 4$, and three times the square of $\mathrm{M} a$ of the circle is the reciprocal of two-thirds of $\mathrm{M} a$ of the circle, or of the bulk of the prime sphere. So the square of M $a 4$ is half of $b a 4$, and the square of $\mathrm{M} a$ of the circle is half of $b a$ of the circle; but such is not the case with any $p$ between the $p 4$ and circle.

The diameter of any regular solid is the diameter of its inscribed sphere; and four times the square of $M a 3$ is the third power of the reciprocal of the bulk of the sphere whose diameter is 1 , namely, 6.75 is the third power of 1.889881 ; and when the diameter of the sphere is 1.889881 its surface will be 12 .

The third power of the entire surface of any prime prism $\div$ by the third power of the lateral surface of such prism gives 3.375 , or twice the square of $\mathrm{M} a 3$. And of course, the third power of the surface of the prime cube $\div$ by the third power of the surface of the prime sphere, gives four times the square of $\mathrm{M} a 3$. 
When the diameter of any given prism is $=d a$ of a like $p$, the bulk of such prism will be the square of the diameter, for the reason that bulk flows by a triplicate ratio to that of diameter, or any other linear measure of the given solid; and in such case, the surface will be six times the diameter, for the reason that surface flows by a duplicate ratio to that of diameter.

We know but little or nothing of the mode of operation of that power or force (better expressed by the word attraction than by any other) which, like a messenger of Omnipresence, operates throughout the solar system, (and by analogy; throughout the universe,) in causing the gravity or ponderosity of the bodies of aggregate matter which compose the solar system, and also in controlling their motions during their eternal rounds.

The phenomena, however, caused by the force of attraction, enable us to contemplate many of its physical effects, and to investigate and determine their ratios and proportions by means of the powers and roots of numbers, whatever the number of elements may be which enter into the calculation, as that of time, space, distance, force, convergency, motion, \&c.

From the phenomena observed in the solar system, which are manifestly caused by the power or force of attraction, we properly conceive the sun to be situated in, or to be the centre of attraction for the solar system; and hence, that all bodies of matter in the solar system, which revolve around the sun, are attracted and retained in their orbits by the sun. Nevertheless, those bodies which revolve around the sun are observed to possess the power of attraction; hence each planet having satellites, attracts and retains them in their orbits.

But a question which may yet require a further and a better consideration of philosophers and astronomers, is, whether a like quantity of matter, wherever situated in the solar system, possesses a like power of attraction, or a like power of attraction for another body of matter; or whether the power of attraction incident to a body of matter situated in the solar system, may not depend upon its distance from the sun, or centre of attraction of the system? ' If the power of attraction of a given quantity of matter be the same, wherever situated, then Jupiter must be far less dense than the earth; but if the power of attraction of a given quantity of matter in the solar system be inversely as the distance of such matter from the sun, or centre of attraction of the system, then the density of the matter of which Jupiter and the earth are composed may be considered as being equal, or substantially so. And in such case we should say that the power of attraction of a given quantity of matter in the solar system is inversely as its distance from the sun, or centre of attraction of 
the solar system. And that this hypothesis is the true, would seem to be well established from the observations and calculations of some of the most astute mathematicians and astronomers, who have found the law to be so general, uniform and exact, as to construct nice astronomical tables upon its correctness.

But it must be manifest to any person, that the theory of the different densities of the planets can have no other support in favor of it, than that of the Newtonian hypothesis, that all matter, wherever situated, is equally endued with an innate or inherent principle of attraction ; which theory, on examination, will perhaps be found not to be supported or sustained by a single fact or phenomenon found to exist in the universe. Nor is it at all certain that the atheistical tendency of such theory was relieved by the vague conjecture that the Almighty originally projected the heavenly bodies in tangents to their orbits, and thereby placed them under the eternal control of this original, innate power of attraction; for such hypothesis only presupposes that attraction had previously aggregated the masses of matter, each of which was waiting for the impulse to be given. Nevertheless, this naked conjecture or hypothesis, wholly unsustained or supported by any physical law or phenomenon with which we are acquainted, is too generally conceived to be a full and ample belief in, or acknowledgement of the wisdom and power of Deity. But I must dissent from those philosophers who attempt to inculcate such notions by mingling them with the doings of a deity of their own making. Such is certainly not an exposition or explanation of natural philosophy, but is emphatically what Newton's admiring biographer alleges, - a creation of natural philosophy.

It is not, perhaps, the better way, in the development of natural philosophy, to reject everything as being vulgar which falls under common observation, and strike at some bold and original hypothesis, as the foundation to which everything must be squared, (or rather equated,) in order that one may have the name of creating an entire system; nor to cast aside as useless all the actual evidences we possess, which can aid us in our investigations, even though such evidence may (by certain philosophers) have been used in a whimsical or futile manner, by attempting to prove more by it in respect to the phenomena of nature than is warranted by true philosophy. Nevertheless, the most common observer must perceive some analogy between the law of attraction, as operating in the solar system, and the operation of the laws of magnetism, electricity, galvanism and electro-magnetism, which we are able to elicit for our use and edification 
from the great store-house or laboratory of nature; which are perhaps but modifications of one great physical law of the universe; all tending, however, to induce the belief that it is an omnipresent law, and is not under the necessity either to wait for its prey, or to reach for it.

The equal innate gravity of matter, wherever situated, necessarily (if adopted as an axiom) draws after it the concomitant theory of the different densities of the planets; and if either hypothesis is true, they both doubtless are. So also if either of them be untrue, they doubtless both are. But to me both hypotheses appear to be wholly uncalled for, and unsupported by any legitimate evidence of which we can avail ourselves; and that in lieu of the round-about process in attempting to ascertain the different densities of the planets having satellites, supposed to exist in consequence of the different attractive powers which they exert upon their respective satellites, in proportion to the bulks or magnitudes of the planets, we have only to consider the quantity of matter in the respective planets to be as their bulks or magnitudes, and that their powers of attraction, in proportion to their respective magnitudes, are inversely as their respective distances from the sun or centre of gravity of the system ; and especially when we are sustained by the evidence, the fact, in accordance with the general and well known law, that their respective relative powers of attraction are given by multiplying their respective bulks or magnitudes by the inverse of their respective distances from the centre of gravity of the solar system; for here is a fact found, according with a law found to be uniform and universal, that the squares of the periods of the planets are as the cubes of their mean distances from the sun.

I am aware that the adoption of my premises would abstract from mankind a vast amount of admiration ; for "still they gazed, and still the wonder grew," that human intellect could become so sublimed as to be able to weigh off the planets; and determine their different densities, when in reality the exploit supposed to have been accomplished, was but a corollary flowing from one of the greatest absurdities that was ever interwoven into a code of philosophy.

Let us now examine a little into the attractive powers of Jupiter and the earth. The time of the period of the earth to that of Jupiter is as 1 to 11.8568; and the mean motion of Jupiter to that of the earth is as 1 to 2.2802 . The mean distance of the earth from the sun to that of Jupiter is as 1 to 5.1993 , and the cube or third power of 5.1993 is the square of 11.8568 ; hence the square of the reciprocal of the rate of mean motion is the mean distance. 
Hence, the mean attractive power of a planet applied to its satellite to retain it in its orbit, is the square of the mean motion of the satellite; hence the whole amount of motion of a satellite during its entire period is the square root of the whole amount of the force of attraction expended upon the satellite during the period. Thus the rate of mean motion, or the square root of the rate of mean force, multiplied by the time of the period, will give the time of the period of the satellite when operated upon by a different rate of force, but at the same distance from the attracting body.

According to LaGrange, the power of attraction of the earth to that of Jupiter, is as 1 to 313.0640 , and the square root of 313.064 is 17.6936 , which of course would be the rate of mean motion of a satellite revolving around Jupiter, if the rate of mean motion of a satellite revolving around the earth at the same distance were 1. The distance of Jupiter's first satellite is found to be a little greater from the centre of Jupiter than the moon is from the centre of the earth. The period of Jupiter's first satellite is one day and .7639 of a day, namely, 1.7639 days.

A. If, then, the time of the period of Jupiter's first satellite be multiplied by the square root of the attractive power of Jupiter, compared with that of the earth, which is assumed at 1 , namely, by 17.6936 , it gives 31.20974 days, which would be the time of the period if such satellite were revolving around the earth at the same distance from the centre that it revolves from the centre of Jupiter. And the square of the period of the moon is to the cube of its mean distance from the earth as the square of 31.2097 is to the cube of the mean distance of Jupiter's first satellite from the centre of Jupiter. Hence the rate of mean motion of Jupiter's first'satellite is as much greater than it would be if revolving about the earth at the same distance, as 17.6936 is greater than 1 ; and this calculation will be found to rest, or to be based upon the principle, that if the bulk or magnitude of the earth be 1 , and its distance from the sun be 1 , and its attractive power be 1, the relative or proportional power of attraction of Jupiter, compared with the earth, will be obtained by multiplying the bulk or magnitude of Jupiter, when compared with the earth, by its inverse distance from the sun when compared with the earth.

If we assume the mean distance of the earth from the sun at $95,000,000$ of miles, and the distance of the moon from the earth at 237,500 miles, the relative distance of the moon from the earth to that of the earth from the sun will be as 1 to 400 . 'These distances are however assumed for the sake of round numbers, the actual mean distance of the moon from the earth being generally stated at 240,000 miles. 
The periodic time of the moon around the earth is to that of the earth round the sun as 1 to 13.3683 . If we then adopt the foregoing assumption as to distance, the mean motion of the moon in its revolution about the earth will be to that of the earth about the sun as 1 to 29.9207 , and in such case, while the square of the mean motion of the moon is 1 , the square of the mean motion of the earth will be 895.2482 ; and in such case the relative power of attraction of the earth upon the moon to that of the sun upon the earth would be as 1 to 895.2482. But if we assume the distance of the moon from the earth at 240,000 miles, the relative attraction of the earth upon the moon to that of the sun upon the earth would be as 1 to \$76.7402. Hence the force of attraction exerted by the sun upon the earth would be some 40,000 times as great as that exerted by the moon upon the earth; nor would this power of attraction exerted by the sun upon the earth have a direst operation in any wise upon the tides.

But I will close this article by a few doctrines relative to the laws of force and motion, with an additional reference to the law of falling bodies.

Twice the motion of a revolving body requires quadruple the force of attraction to retain the planet in its orbit.

The period of any planet is the square root of the cube of its mean distance from the centre of gravity.

If $\mathrm{A}$ denote a planet at the distance 1 , and $\mathrm{B}$ a planet at the distance 2 , the time in which $\mathrm{B}$ will revolve over a space equal to the circumference of A's orbit, will be $=$ to the square root of B's distance, or of the diameter of A's orbit. Hence the time in which $B$ will revolve over a space = to the circumference of A's orbit, will be inversely as the rate of the mean motion of $B$.

Hence the rate of mean motion of a planet as proportioned to the mean distance, is inversely as the square root of the mean distance; or the square root of the mean distance is the inverse of the rate of mean motion. Hence the mean distance is a mean proportional between the time of the period and the square root of the mean distance.

If $\mathrm{D}$ denote another planet whose mean distance is 4 , the time required for $D$ to pass over a space equal to the circumference of A's orbit will be equal to the square root of D's distance. Hence if ever so many planets revolve round the same central force, and the mean distance of either of them be assumed at unity, the time required for any other : planet to revolve over a space equal to the circumference of the orbit whose distance is 1 , will be equal to the square root of the mean distance of the planet, compared with that whose distance is 1 . This, however, can only be true in respect to centric orbits. 
So when the mean distance is 1 , the rate of mean motion is $=$ to the time of the period. When the distance is 2 the rate of mean motion is equal to one fourth of the period. When the distance is 3 the rate of mean motion is equal to one ninth of the period; and so on. Hence, if the force of attraction be inversely as the distance, it is the square of the mean motion; but if the force of attraction be inversely as the square of the distance, it will be the fourth power of the mean motion.

If we take two planets of a system, as $B$ and $D$, if the distance of $B$ is the square root of the distance of $D$, the period of $B$, the rate of motion of $B$, and the rate of force of $B$, will respectively be the square root of like elements of $D$.

If the mean distance of $\mathrm{A}$ be 1 , and that of $\mathrm{D}$ be 4 , if the force of gravity varies inversely as the distance, $D$ will receive twice the force in the time of its period that $A$ will in the time of its period. But if the force of gravity varies inversely as the square of the distance, $D$ will receive but half the force in the time of its period that $A$ will in the time of A's period.

The mean distance of a planet multiplied by the square root of the mean distance, gives the period; and the reciprocal of the period of a planet is the product of the rate of motion by the rate of force. Hence when the circumference of the orbit is 16 , and the time of the period is 4 , the radius of the orbit or the distance is then the third root of 16 , and the square root of such radius is the third root of four; and the product of such radius by its square root gives the time of the period, or one fourth of the circumference of the orbit. But when the period is 8 , the product of radius by the square root of radius, gives as well the diameter as the period. Or the version may be thus, - the cube root of the period multiplied by the cube root of the square of the period gives the period.

By the popular determination of the quadrature, when the period of the planet is $=$ one fourth of the circumference of the orbit, such period is 2 below 4 ; for when the period is 4 by the popular mode, one fourth of the circumference of the orbit is $\frac{2}{3}$ below 4 . Hence by any system of the quadrature, when the period of the planet is = one fourth of the circumference of the orbit, the time of the period will be the third power of twice $\mathrm{M} a$ of the circle.

So by any system, when the period is = one fourth of the circumference of the orbit, the circumference of the orbit is half of the third power of the circumference of the circle whose diameter is 1 .

Nevertheless, by the popular determination of the quadrature, if the period of a planet be made commensurable with the diameter of the orbit in the powers and roots of definite quan. 
tities, it will not be commensurable with the circumference of the orbit, or with any part of it; and if the period be made commensurable with the circumference of the orbit, or with any part of it, (namely, with the path in which the planet travels,) it will not be commensurable with the diameter. But by the true determination of the quadrature of the circle, the period of a planet is as well commensurable with the circumference of its orbit as with its diameter.

I have heretofore alleged, that by the true law of gravity or attraction, the earth would exert quadruple the amount of the force of attraction upon the moon, that would be exerted by the Newtonian law; and hence the true law would be just what seemed to be required by Clairaut, Euler and others, for the accounting for the motion of the moon's apogee, upon the principle of attraction. So by the true law of gravity; any planet in the solar system will be attracted towards the sun by quadruple the force that it would be by the Newtonian law.

And lest any one should not readily apprebend the truth of the allegation, I will shortly explain, nevertheless, some part of the explanation may appear somewhat paradoxical, or similar to Maclaurin's explanation of what would be the various movements of the planets were they operated upon by various laws of gravity which have no existence in nature. Nevertheless, by all philosophy, it requires quadruple the force of attraction to balance duplicate the motion; and the motion of a planet is doubled at one fourth the distance, for the reason that the force is quadrupled. If, then, the Newtonian law of gravity gave but just one half the force requisite for the motion of the moon, or of the moon's apogee, as found by Clairaut, Euler and others, then in order that the Newtonian law might give the actual or observed motion, it would be necessary for the moon to revolve at one fourth the distance from the earth that it now does. And the like in respect to any planet in the solar system; the motion being just double that which would be sustained by the Newtonian law of gravity at the distance at which the planet revolves. And in order that it might revolve about the sun retaining the motion it now has, it must revolve at one fourth the distance it now does, in order to be sustained in its orbit by the Newtonian law of gravity.

In respect to a body commencing its fall towards the earth, as there is no portion of the fall, from its commencement, however small, in which the fall or motion is not accelerated, it follows as a consequence to such axiom or proposition, that at any given point of space of the fall, or at any given instant of time during the fall, the amount of space then fallen over is the square 
of the amount of force then expended on the body during the fall, and consequently that the intensity of the force varies inversely as the distance varies.

Nothing could be devised to show more clearly the truth which I allege, than the simplest formula that can be constructed for the purpose of exhibiting the numerical law of falling bodies. If we conceive a body to fall from a state of rest over a given space in a given time, (as for instance, over one rod in one second of time,) we may denote the operation by

\section{1}

the space fallen over, (denoted by the lower figure 1,) being a unit of space, and its square root denoted by the upper figure 1 , will as well denote the unit of force employed during the fall over the unit of space, as it will the unit of time in which the body is falling over the unit of space.

If we now conceive the unit of space to be divided into four equal parts, the formula will be thus,

$$
\begin{array}{lll}
1 & 2 & 1 \\
1 &
\end{array}
$$

in which case the odd numbers in the lower series will denote the four equal parts into which the unit of space is divided. If then we extract the square root of the lower series, taking figure 1 for the first period in the process of extraction, the square root of the lower series will be expressed by figure 1 over each odd number of the lower series; which square root expressed by the upper series, will denote as well a division of the unit of force, as a division of the unit of time, into equal parts. Hence, while the body is falling over that portion of the unit of space denoted by figure 1 in the lower series, one half of the unit of force, as also one half of the unit of time, will be expended; and the remaining half of the unit of force, and also of the unit of time will be expended while the body is falling over three fourths of the unit of space, namely, that denoted by figure 3 .

So the unit of space may be divided into as many equal parts as we please, even to an infinite or indefinite number; in which case the lower series of the formula will be expressed by the natural numbers in their order, while the equal parts of such unity of space will be denoted by the odd numbers of the series; and a like extraction of the square root of the series, (taking figure 1 at the left as the first period in the process of extraction,) will give figure 1 over each odd number, as the square root of the series; in which case the unit of force, as also the unit of time, will be divided into as many equal parts as there are figures in such root. And it is manifest that however far we extend such 
division, at any instant or point of the fall, - no matter how little space has been described or fallen over, - the sum of the odd numbers of the lower series described by the fall thus far, will be the square of the number of equal moments, or of the equal amounts of force which have been expended thus far. Hence the space passed over or described in the fall is as well the square of the amount of force, as of the amount of time expended ; and consequently there is no space described or passed over, without acceleration.

Such, then, enables us, in the most simple and easy manner, to determine the law of gravity, namely, by comparing the descent of two bodies falling from a state of rest, but from different distances from the same centre of gravity.

\section{SUGGESTIONS IN REGARD TO CYLINDRICAL SECTIONS.}

I will here only suggest in respect to the three principal planes that may be cut from the prime cylinder, or cylinder, the diameter of whose inscribed sphere is unity or 1 .

If we conceive the prime cylinder standing upon one end, upon a horizontal plane, the base will denote the prime circle, and if such cylinder be cut horizontally through, the plane will be the prime circle; and if a plane be cut perpendicularly through the centre of the cylinder, the plane will be the prime square.

If the cutting plane commence on the side of the cylinder, at any point between the base and altitude, and terminate on the opposite side, at the point touching the periphery of the base, the plane will be an ellipse (or elongated circle,) the area of which will be the product of the longest by the shortest diameter, multiplied by the area, or one fourth of the circumference of the prime circle. The longest diameter of such ellipse may be said to have its upper and lower ends; the upper end being the one above the base of the cylinder.

As the shortest diameter of any ellipse cut from the prime cylinder is always unity, it is therefore manifest that the area of any such ellipse is to the longest diameter, as $\mathbf{M} a$ of the circle is to 1 , or as 1 is to $d a$ or $b a$ of the circle ; and hence, that the area will increase or decrease by the same ratio that the longest diameter will; the consequence is, that the longest diameter (or major axis) of any ellipse cut from the prime cylinder, multiplied by the area of the prime circle, gives the area of such ellipse; consequently, if the major axis of such ellipse be $=d a$ or $b a$ of the circle, the area of such ellipse will be 1 , or is $=m$ of the circle whose area is $=d$ or $b$ of the circle. Thus, the area of 
any ellipse cut from the prime cylinder, is $=m$ of a circle whose $b$ or $d$ is = the major axis of such ellipse; and hence, if the major axis be at its maximum, namely, the square root of 2 , the area of such ellipse is necessarily a mean between $M a$ and twice $\mathrm{M} a$ of the circle; and such ellipse may properly be termed the prime ellipse, the area of its circumscribed circle, or of any circumscribed parallelogram, being double that of a like inscribed figure. The plane of such ellipse as situated in the cylinder, forms an angle of $45^{\circ}$ with the base; and as the focal distance of any ellipse cut from the prime cylinder is $=$ the altitude of the upper end of the longest diameter, or major axis, hence, the focal distance of the prime ellipse is unity. And as the length of the describing thread of any ellipse is $=$ the major axis, plus the focal distance, hence the length of the describing thread of the prime ellipse is $=$ the sum of the longest and shortest diameters, or of the major and minor axes. Thus, while the major axis of the prime ellipse is $=b a 8$, the length of the describing thread, is twice $d a 8$, consequently, from one end of the prime ellipse, to the nearest focus, is $=v a 8$ - for in respect to any $p, d a$ plus $v a$ is $=b a$.

So the area of the prime ellipse is $=b$ or $d$ of the circle, whose area is $1-$ or is $=b$ of the $p 8$ whose area is the reciprocal of $b$ - or it is a mean between the area of the square whose circumference is 4 , and the area of the circle whose circumference is 4 .

But it is not my intention here, to treat of the qualities of ellipses; nor to do more than to suggest some unity of purpose in their consideration, which is certainly to be desired; if, (as is generally supposed,) planets can revolve in ellipses, however eccentric - or in ellipses of extreme eccentricity, and in which, (according to the modern version of Kepler's law) "the squares of the periods are as the cubes of their semi-major axes." Now, according to this version, if we conceive two planets to revolve around the same central force in orbits, the respective circumferences of which are 4, one orbit being a circle (either centric or eccentric,) and the other an ellipse of extreme ellipticity. In such case, the time of the period of the planet revolving in the ellipse must be 1 , while that in the circle will be .5 . Hence, in the ellipse, the time of the period will have no regard to the space, or length of road passed over, or to the area described by the radius vector, or even to the amount of force of attraction expended on the planet during the period. And if we conceive a planet to revolve in an ellipse, we must conceiye it to revolve without obeying any of the requirements of the great physical law of the universe. 
But in respect to the circular orbit, (whether centric or eccentric,) by any system of the quadrature, if the diameter of the orbit be $=b a$ of any given $p$, the time of the period will be the cube or third power of $\mathrm{B}$ of a like $p$. So also, if the diameter is $=d a$ of any given $p$, the time of the period is the cube or third power of $\mathrm{D}$ of a like $p$. So when the diameter is equal $d a$ or $b a$ of the circle, the period is the cube, or third power of $\mathrm{B}$ or of $\mathrm{D}$ of the circle.

Hence, the reciprocal of $\mathbf{A}$ of any given $p$, is the diameter of an orbit in which the period is the third power of $\mathrm{B}$ of a like $p$. And the reciprocal of $\mathrm{M} a$ of any $p$, is the diameter of an orbit in which the time of the period is the third power of $D$ of a like $p$, by any system of the quadrature.

Hence, the time of the period of a planet, is the third power of the square root of radius of the orbit.

So if the diameter of an orbit be $=m$ of the circle, whose area is $2,-$ namely, $=b$ of the equal square, or square whose area is = to the area of the prime circle, or whose diameter is one eighth of the square of the circumference of the prime circle, the time of the period in such orbit will be the sixth power of $m$, or the third power of the area of such equal square. 



THIS BOOK IS DUE ON THE LAST DATE STAMPED BELOW

AN INITIAL FINE OF 25 CENTS WILL BE ASSESSED FOR FAILURE TO RETURN THIS BOOK ON THE DATE DUE. THE PENALTY WILL INCREASE TO 50 CENTS ON THE FOURTH DAY AND TO \$1.00 ON THE SEVENTH DAY OVERDUE.

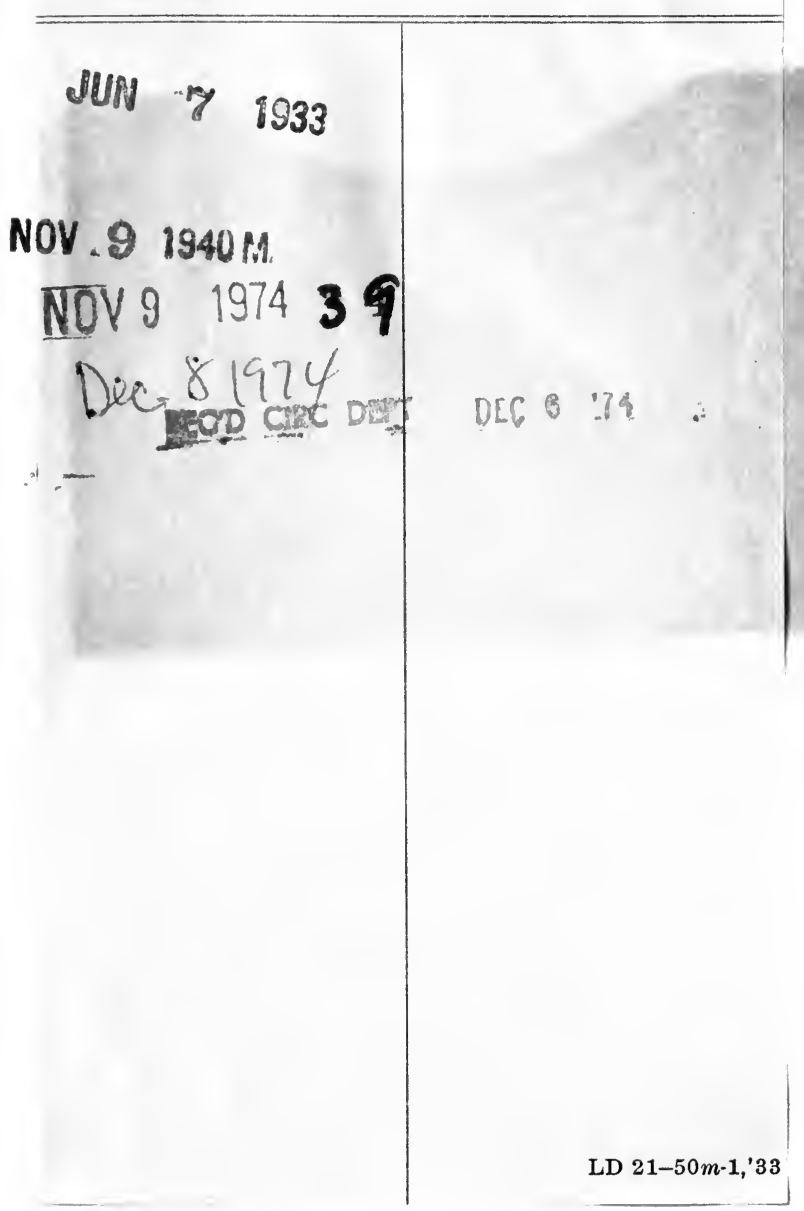




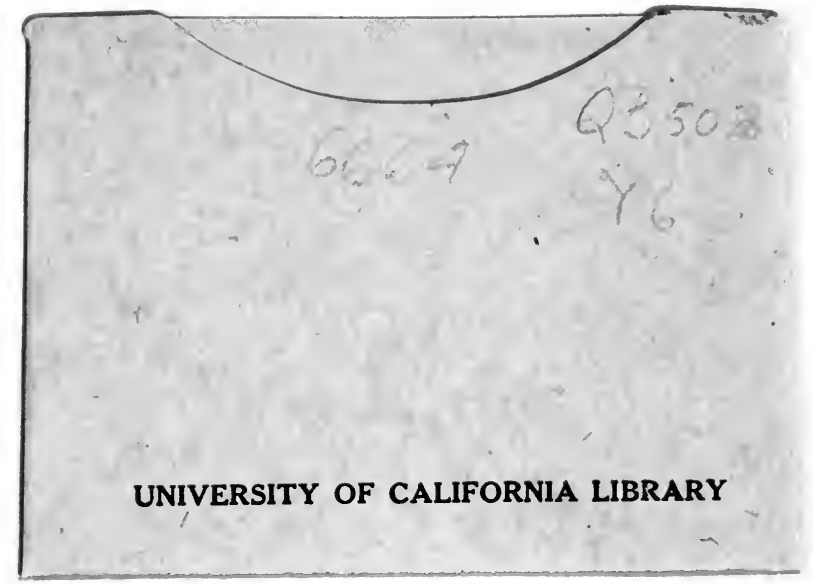


Tite

5 